\title{
Extracellular Matrix Mediated Cartilage Regeneration: A Novel Three-Dimensional In Vitro Microenvironment to Facilitate Cell Expansion and Chondrogenesis
}

\author{
Fan $\mathrm{He}$ \\ West Virginia University
}

Follow this and additional works at: https://researchrepository.wvu.edu/etd

\section{Recommended Citation}

He, Fan, "Extracellular Matrix Mediated Cartilage Regeneration: A Novel Three-Dimensional In Vitro Microenvironment to Facilitate Cell Expansion and Chondrogenesis" (2010). Graduate Theses,

Dissertations, and Problem Reports. 4604.

https://researchrepository.wvu.edu/etd/4604

This Dissertation is protected by copyright and/or related rights. It has been brought to you by the The Research Repository @ WVU with permission from the rights-holder(s). You are free to use this Dissertation in any way that is permitted by the copyright and related rights legislation that applies to your use. For other uses you must obtain permission from the rights-holder(s) directly, unless additional rights are indicated by a Creative Commons license in the record and/ or on the work itself. This Dissertation has been accepted for inclusion in WVU Graduate Theses, Dissertations, and Problem Reports collection by an authorized administrator of The Research Repository @ WVU.

For more information, please contact researchrepository@mail.wvu.edu. 


\title{
Extracellular Matrix Mediated Cartilage Regeneration:
}

\section{A Novel Three-Dimensional In Vitro Microenvironment to Facilitate Cell Expansion and Chondrogenesis}

\author{
Fan He \\ Dissertation submitted to the \\ School of Medicine \\ at West Virginia University \\ in partial fulfillment of the requirements \\ for the degree of \\ Doctor of Philosophy \\ in \\ Exercise Physiology \\ Ming Pei, M.D., Ph.D., Chair \\ Stephen E. Alway, Ph.D. \\ Fred L. Minnear, Ph.D. \\ David L. Williamson, Ph.D. \\ Hanting Zhang, M.D., Ph.D. \\ Department of Exercise Physiology \\ Morgantown, West Virginia \\ 2010
}

Keywords: Mesenchymal stem cells, Chondrocytes, Nucleus pulposus,

Extracellular matrix, Chondrogenesis 


\section{ABSTRACT \\ Extracellular Matrix Mediated Cartilage Regeneration: A Novel Three-Dimensional In Vitro Microenvironment to Facilitate Cell Expansion and Chondrogenesis}

\section{Fan He}

Joint articular cartilage is vulnerable for degenerative disease after trauma due to the limited self-healing ability. Mesenchymal stem cells (MSCs) are considered a promising source for cartilage repair and regenerative medicine because of their excellent capacities of self-renewal and multipotent differentiation. Conventional in vitro expansion system, however, limits the utilization of MSCs for cartilage regeneration because in monolayer culture MSCs rapidly lose their self-renewal ability and differentiation potential. The lack of proper microenvironment in the current two-dimensional expansion system is attributed to the loss of self-renewal and multipotent differentiation in MSCs. Three-dimensional (3D) cell-deposited extracellular matrix (ECM) provides a novel strategy for in vitro MSC expansion, not only promoting cell proliferative rate but also improving chondrogenic potential. We generated 3D ECM deposited by synovium-derived stem cells (SDSCs) to reconstruct an in vitro 3D stem cell niche. First, MSCs were expanded on 3D ECM including SDSCs, adipose-derived stem cells (ADSCs) and bone marrow stem cells (BMSCs). The cell morphology of MSCs cultured on ECM changed to a thin and spindle-like shape and cell migration was directional along the fibrils of ECM. A dramatic increase in cell number and a greatly enhanced chondrogenic potential were observed, though surprisingly the ECM-treated MSCs did not display concomitantly improved adipogenic or osteogenic potentials. 3D ECM was also adopted to expand terminally differentiated cells including articular chondrocytes and nucleus pulposus (NP) cells, in order to improve the technique of autologous cell transplantation for cartilage repair and intervertebral disc regeneration. We found that ECM not only improved chondrocyte or NP cell expansion but also delayed dedifferentiation. Chondrocytes or NP cells expanded on ECM also acquired a strong redifferentiation capacity, particularly when treated with TGF- $\beta 1$ in a pellet culture system. Further, we compared the activity of several key signaling kinases and integrin expression in BMSCs cultured on ECM and conventional plastic flasks. 3D ECM increased the expression of SSEA-4, integrin $\alpha_{2}, \alpha_{4}$ and $\beta_{5}$, and induced sustained activation of Src kinase, ERK1/2 and cyclin D1. Not only the expression but also the responsiveness of TGF- $\beta$ type II receptor was enhanced by 3D ECM in BMSCs. These observations suggested that 3D cell-deposited ECM promoted BMSC proliferative capacity through integrin/Src/ERK1/2 signaling pathway and enhanced chondrogenic potential by improving the responsiveness of TGF- $\beta$ receptors. Finally we generated two distinct types of ECM separately deposited by SDSCs and articular chondrocytes. SDSC-deposited ECM (S-ECM) contained fibrillar type I collagen, whereas chondrocyte-deposited ECM (C-ECM) is composed of fibrillar type I and II collagen with proteoglycans. S-ECM showed a superior influence on SDSCS to C-ECM in cell self-renewal and chondrogenic potential. The proliferative and chondrogenic abilities of chondrocytes were comparable on S-ECM and C-ECM. SDSC-deposited ECM was superior to 
chondrocyte-deposited ECM and functioned as a universal expansion system in vitro for MSCs and terminally differentiated cells to promote cell proliferative and chondrogenic potentials. In conclusion, we demonstrated that 3D cell-deposited ECM provides a proper microenvironment for in vitro expansion of MSCs and terminally differentiated cells, not only promoting self-renewal ability but also improving chondrogenic potential, and the novel 3D expansion system greatly improves cell-based cartilage and intervertebral disc regeneration. 


\section{LIST OF ABBREVIATIONS}

2D

3D

ACT

ADCT

ADSCs

AECM

$\mathrm{AF}$

ALP

BMP

BMSCs

cbfa-1

C-ECM

C/EBP $\alpha$

Col I

Col II

Col X

Co-Smads

DAB

DCFH-DA

DDRs

DMMB

DNA

ECM

EGF

ESCS

ERK

FACS
Two-dimensional

Three-dimensional

Autologous chondrocyte transplantation

Autologous disc cell transplantation

Adipose-derived stem cells

ADSC-deposited ECM

Annulus fibrosus

Alkaline phosphatase

Bone morphogenic protein

Bone marrow stem cells

Core binding factor alpha 1

Chondrocyte-deposited ECM

CCAAT/enhancer binding protein alpha

Type I collagen

Type II collagen

Type X collagen

Common-partner Smads

3,3'-diaminobenzidine

2',7'-dichlorofluorescein-diacetate

Discoidin domain receptors

Dimethylmethylene blue

Deoxyribonucleic acid

Extracellular matrix

Epithelial growth factor

Embryonic stem cells

Extracellular signal regulated kinase

Fluorescence-activated cell sorting 


\begin{tabular}{|c|c|}
\hline FAK & Focal adhesion kinase \\
\hline FBS & Fetal bovine serum \\
\hline FGF & Fibroblast growth factor \\
\hline FITC & Fluorescein isothiocyanate \\
\hline GAGs & Glycosaminoglycans \\
\hline GSC & Germ stem cell \\
\hline $\mathrm{GuHCl}$ & Guanidine hydrochloride \\
\hline hESCs & Human embryonic stem cells \\
\hline HIF & Hypoxia-inducible factors \\
\hline HSC & Hematopoietic stem cell \\
\hline IGF & Insulin-like growth factor \\
\hline IPFP & infrapatellar fat pad \\
\hline iPS cells & Induced pluripotent stem cells \\
\hline IL & Interleukin \\
\hline I-Smads & inhibitory Smads \\
\hline IVD & Intervertebral discs \\
\hline JNK & c-Jun $\mathrm{NH}_{2}$-terminal kinase \\
\hline LBP & Low back pain \\
\hline LPL & Lipoprotein lipase \\
\hline MAPK & Mitogen activated protein kinase \\
\hline $\mathrm{MFI}$ & Mean fluorescent intensity \\
\hline MKK & MAP kinase kinase \\
\hline MMP & Matrix metalloproteinases \\
\hline mRNA & Messenger ribonucleic acid \\
\hline MSCs & Mesenchymal stem cells \\
\hline NP & Nucleus pulposus \\
\hline OA & Osteoarthritis \\
\hline OCN & Osteocalcin \\
\hline PAK & p21-activated kinase \\
\hline
\end{tabular}


PBS

PCR

PEG

PGA

PI3K

PKC

PLA

PLGA

PPAR

PTHrP

RGD

ROS

R-Smads

RT

Runx2

RWVs

SDSCS

S-ECM

SEM

$\mathrm{SH} 2$

Sox9

SPP1

Src

SSEA

SZP

TE

TGF- $\beta$

TGF- $\beta$ RII

VEGF
Phosphate buffered saline

Polymerase chain reaction

Poly ethylene glycol

Poly glycolic acid

Phosphatidylinositol 3-kinase

Protein kinase $\mathrm{C}$

Poly lactic acid

Poly lactic-co-glycolic acid

Peroxisome proliferator-activated receptor gamma

Parathyroid hormone-like peptide

Arginine, glysine and aspartic acid

Reactive oxygen species

Receptor-regulated Smads

Reverse transcriptase

Runt domain transcription factor

Rotating wall vessels

Synovium-derived stem cells

SDSC-deposited ECM

Scanning eectron microscope

Src homology 2

SRY (sex determining region Y)-box 9

Secreted phosphoprotein type 1

v-src sarcoma (Schmidt-Ruppin A-2) viral oncogene homolog

Stage-specific embryonic antigen

Surface zone protein

Tissue engineering

transforming growth factor- $\beta$

TGF- $\beta$ receptor II

Vascular endothelial growth factor 


\section{TABLE OF CONTENTS}

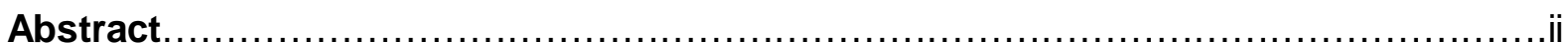

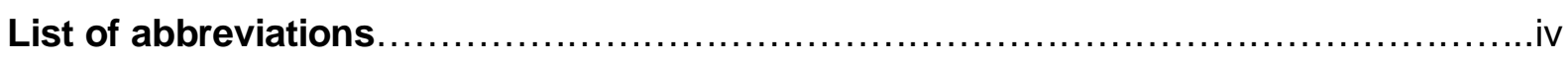

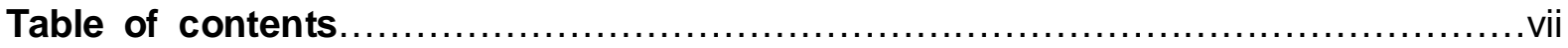

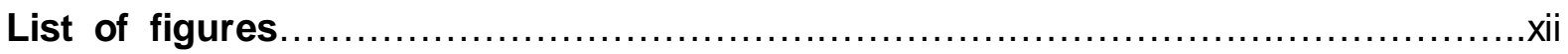

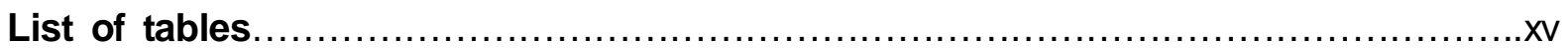

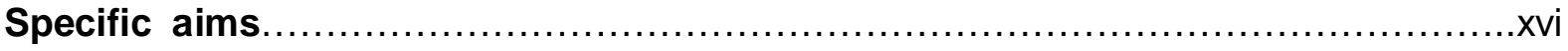

\section{Chapter 1. Literature review}

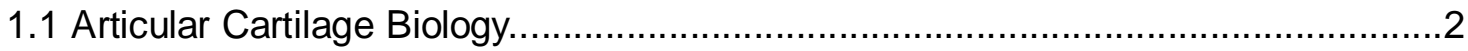

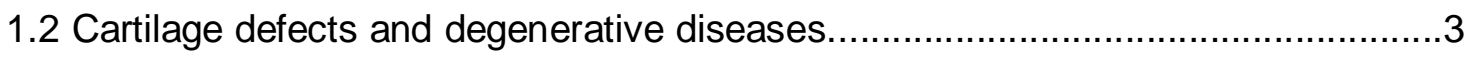

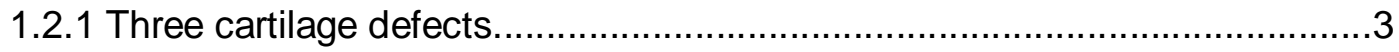

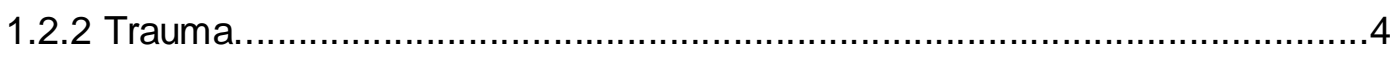

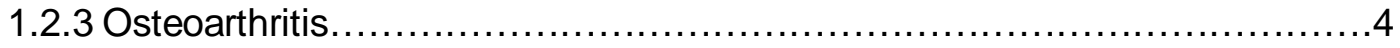

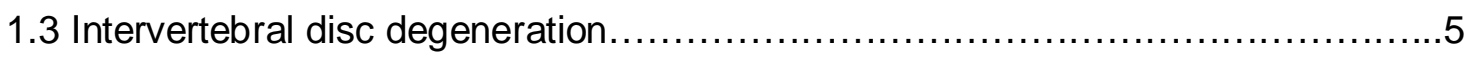

1.4 Current therapy tragedies for articular cartilage repair and IVD degeneration......7

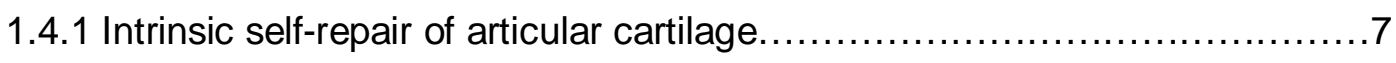

1.4.2 Conservative treatments and surgical procedures.......................

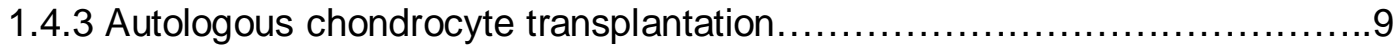

1.4.4 Autologous or allogeneic cell transplantation for nucleus pulposus repair...10

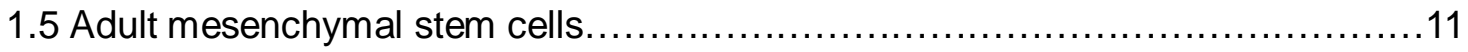

1.5.1 Embryonic stem cells and induced pluripotent stem cells................11

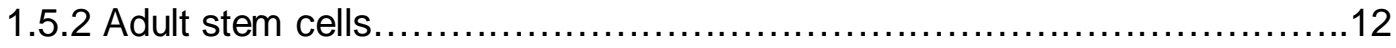

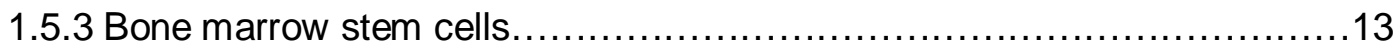

1.5.4 Adipose-derived stem cells.............................................

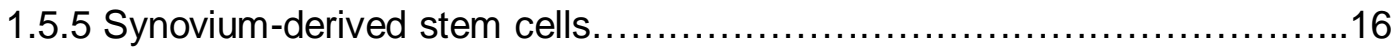

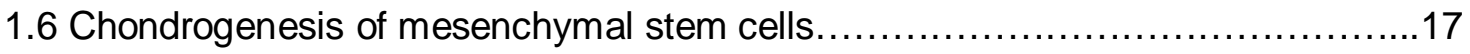

1.6.1 Growth factors in chondrogenesis.......................................

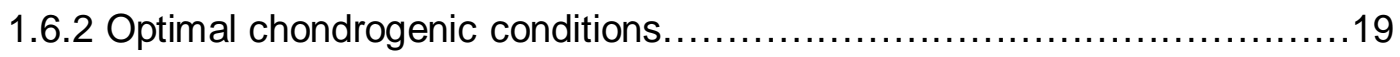

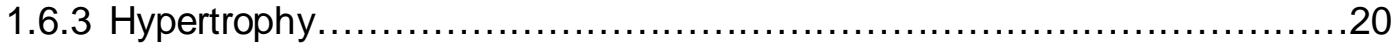


1.6.4 Chondrogenesis of SDSC induced by the combination of TGF- $\beta 1$, IGF-I and FGF-2.

1.6.5 Intracellular signaling pathways of TGF- $\beta$ induced chondrogenesis..........21

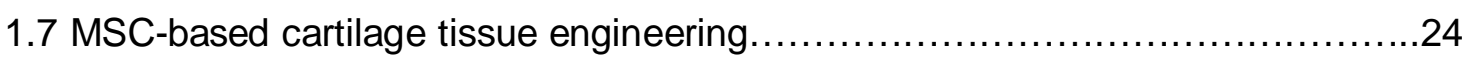

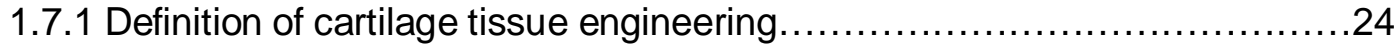

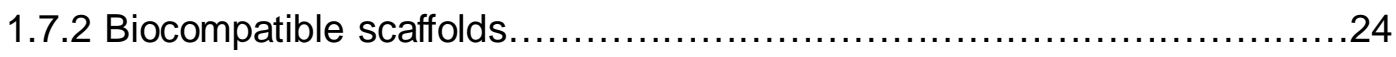

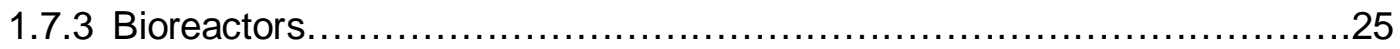

1.7.4 Cartilage tissue engineering using synovium-derived stem cells...........26

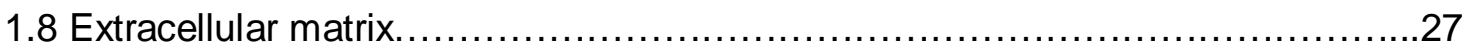

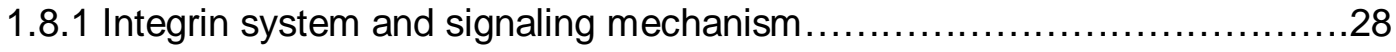

1.8.2 Typical ECM proteins and their integrin receptors......................30

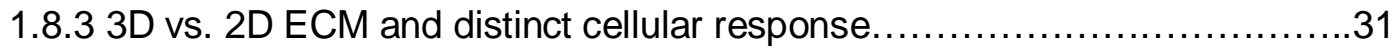

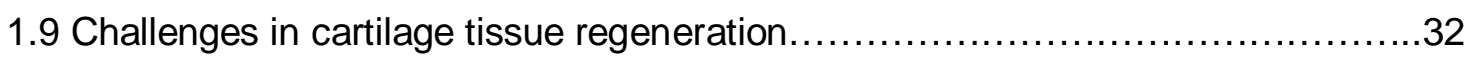

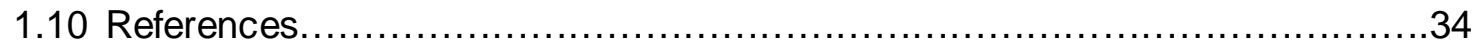

Chapter 2. Reconstruction of an In Vitro tissue-specific microenvironment to rejuvenate synovium-derived stem cells for cartilage tissue engineering

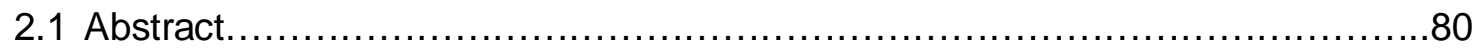

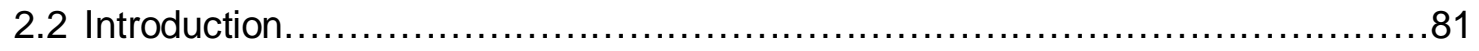

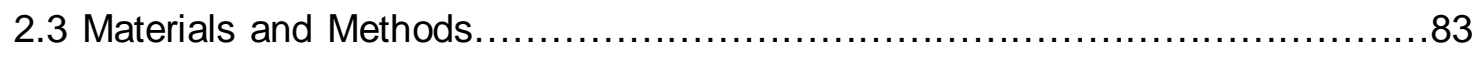

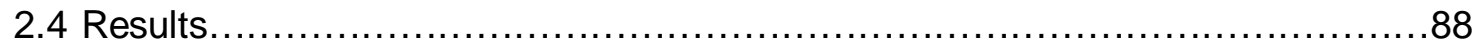

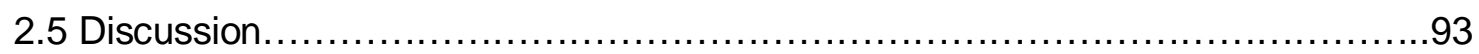

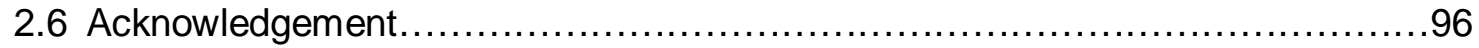

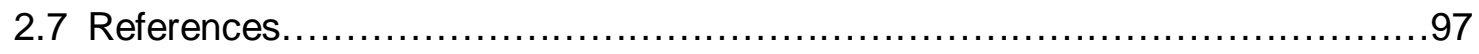

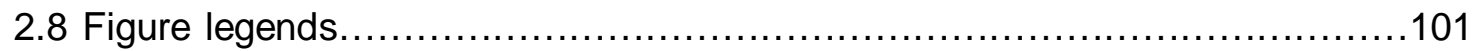

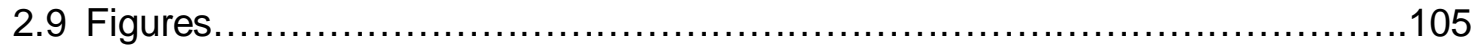

Chapter 3. In Vitro three-dimensional extracellular matrix reprograms adipose stem cells from infrapatellar fat pad toward chondrogenesis

3.1 Abstract 


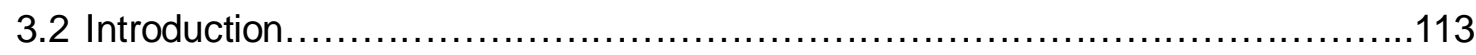

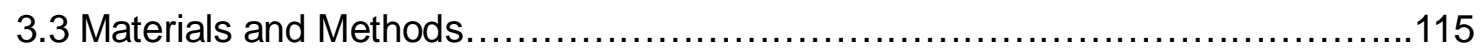

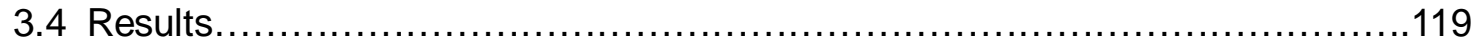

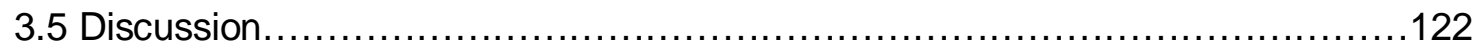

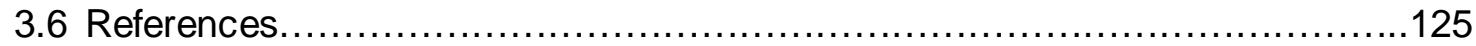

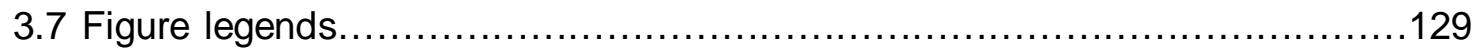

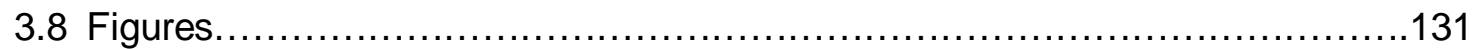

Chapter 4. Extracellular matrix deposited by synovium-derived stem cells delays chondrocyte dedifferentiation and enhances redifferentiation

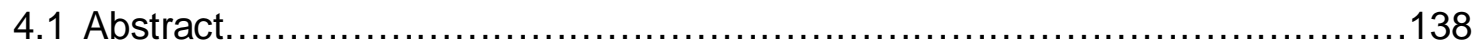

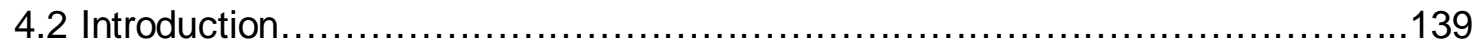

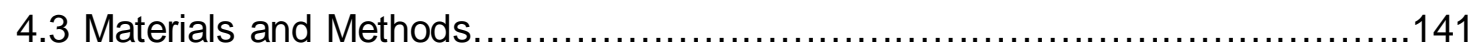

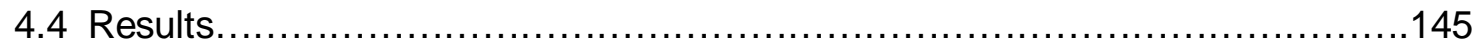

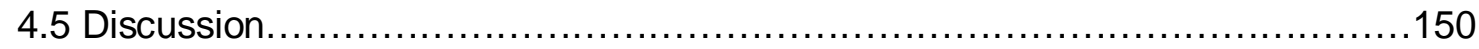

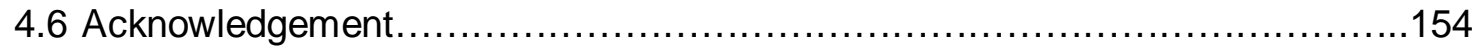

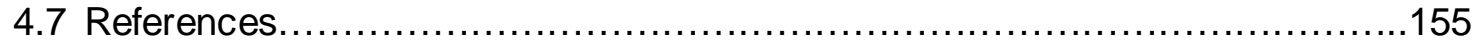

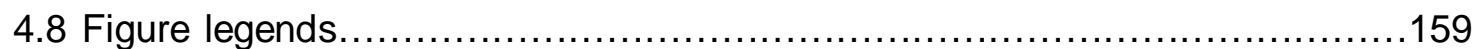

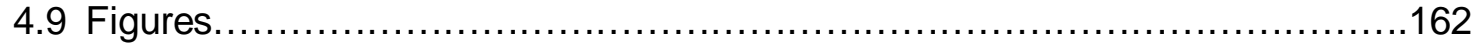

Chapter 5. Rejuvenation of nucleus pulposus cells using extracellular matrix deposited by synovium-derived stem cells

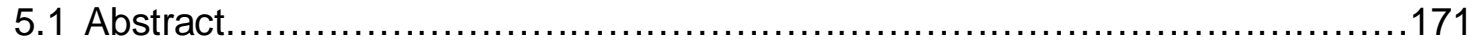

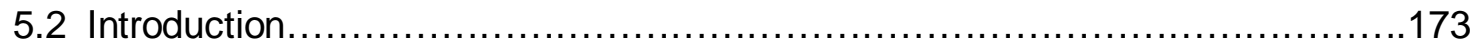

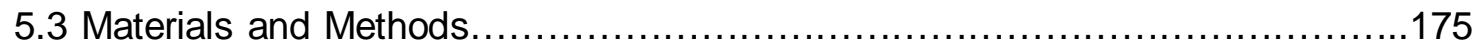

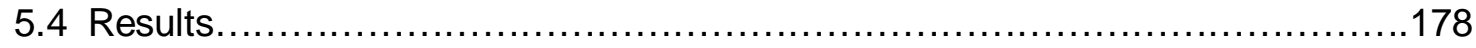

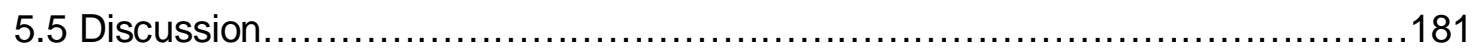

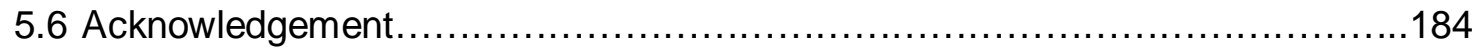

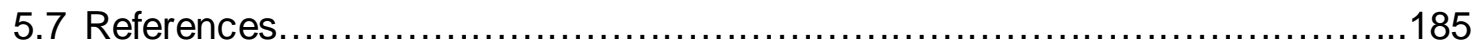

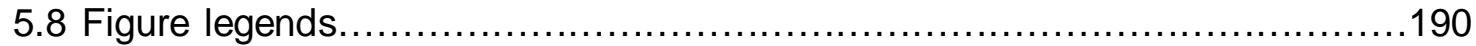


Chapter 6. Three-dimension extracellular matrix deposited by human bone marrow stem cells promotes cell proliferation and chondrogenesis

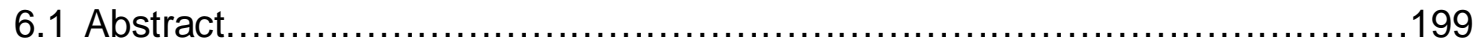

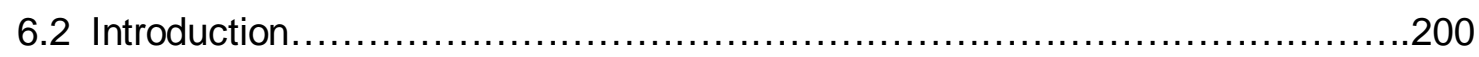

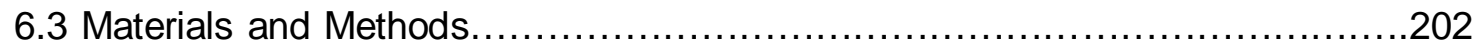

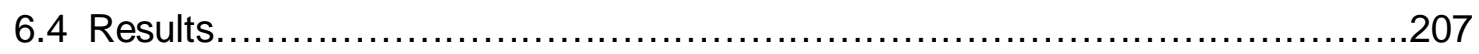

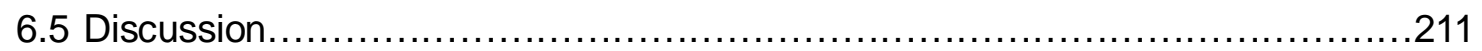

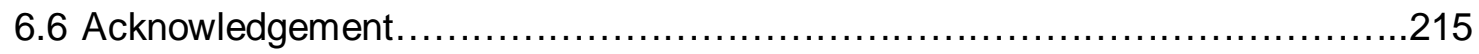

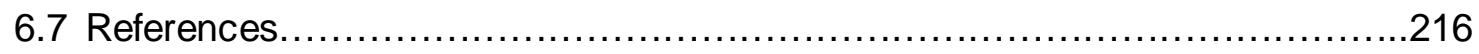

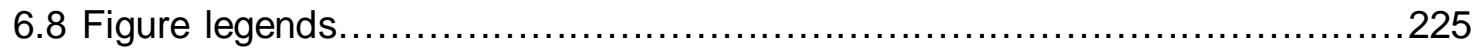

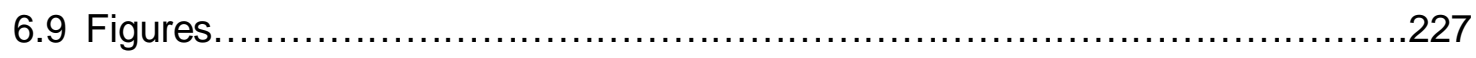

Chapter 7. Comparison of three-dimensional extracellular matrices deposited by synovium-derived stem cells or articular chondrocytes

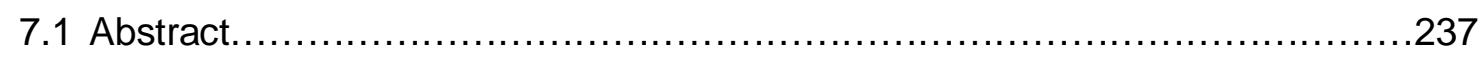

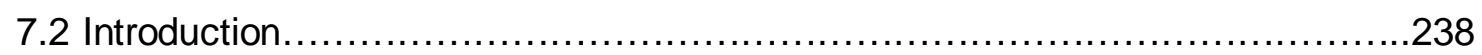

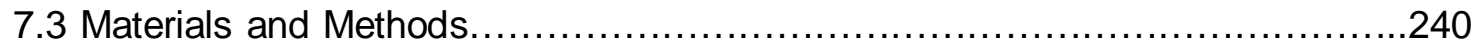

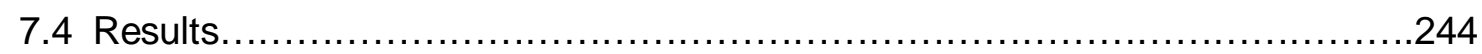

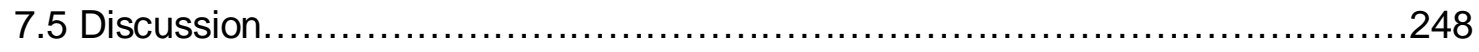

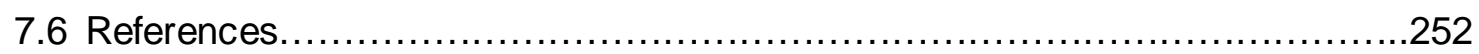

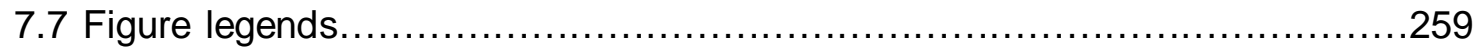

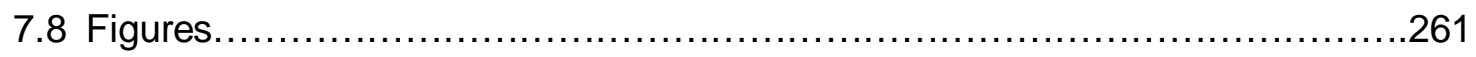

\section{Chapter 8. General Discussion}

8.1 Extracellular matrix improves MSC self-renewal...............................269

8.2 3D cell-deposited ECM is different from 2D reconstructed ECM $\ldots \ldots \ldots \ldots \ldots \ldots \ldots \ldots \ldots$

8.3 The interaction between integrin system and 3D ECM.......................274

8.4 3D ECM is a potential positive-isolation model for MSCs.....................278 
8.5 MSCs expanded on ECM may have higher potential of integration...............279

8.6 3D ECM reduces chondrogenic hypertrophic risk in MSCs....................280

8.7 ECM Improves chondrogenic potential of MSCs and the underlying

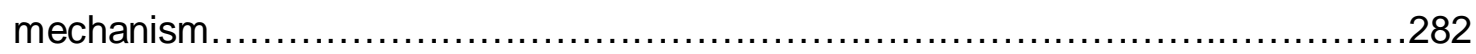

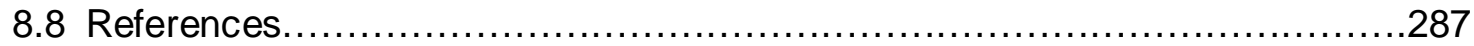




\section{LIST OF FIGURES}

\section{Chapter 1}

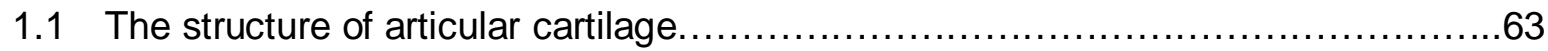

1.2 Histology and immunohistochemistry of hyaline cartilage tissue $\ldots \ldots \ldots \ldots \ldots \ldots \ldots \ldots 4$

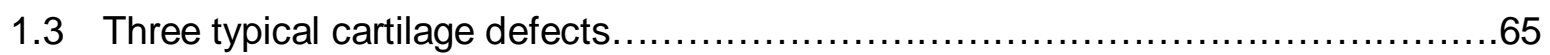

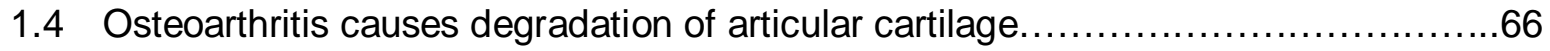

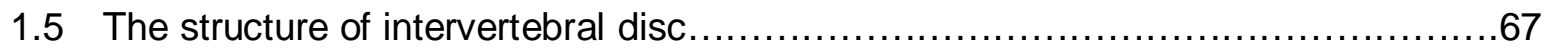

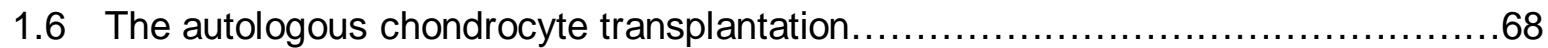

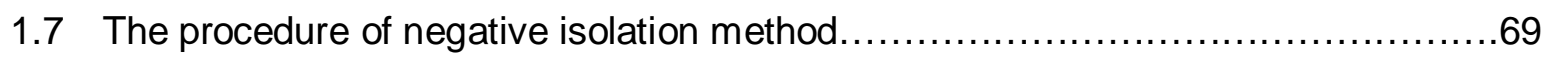

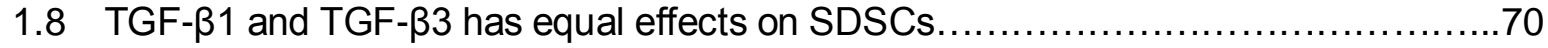

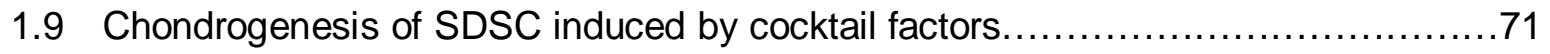

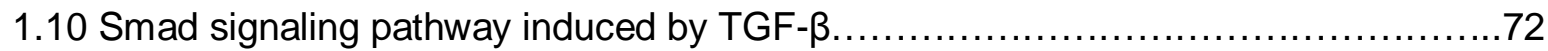

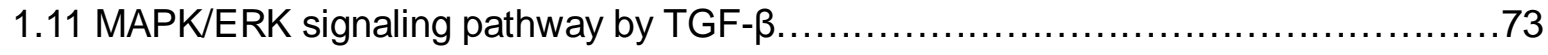

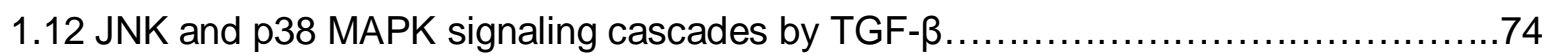

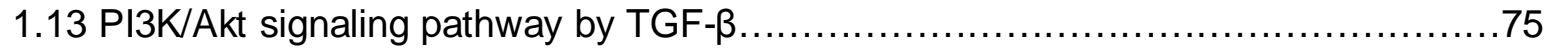

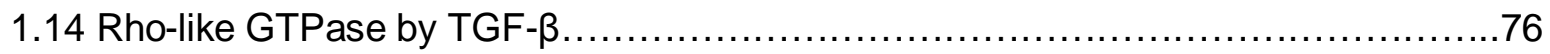

1.15 engineered hyaline cartilage induced by SDSC constructs in vitro $\ldots \ldots \ldots \ldots \ldots \ldots \ldots .77$

1.16 Repair the full-thickness defects by engineered constructs using SDSCs............78

\section{Chapter 2}

2.1 Effect of SDSC-derived ECM on SDSC morphology and proliferation...............105

2.2 Analysis of typical cell surface antigens in SDSCs.............................

2.3 Distinctively chondrogenic capacity from SDSCs grown on SDSC-derived ECM...107

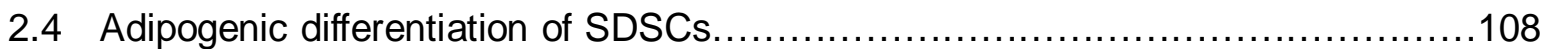

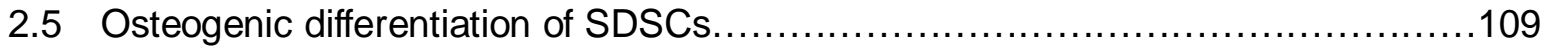

\section{Chapter 3}

3.1 The morphology and proliferative rate of ASCs on Plastic, AECM, SECM ..........131

3.2 Histology and immunohistochemistry of chondrogenic ASC-pellets.................132

3.3 Biochemical analysis of chondrogenic ASC-pellets................................

3.4 Real-time PCR of chondrogenic markers in chondrogenic ASC-pellets.............134 
3.5 Real-time PCR of hypertrophic markers in chondrogenic ASC-pellets...............135

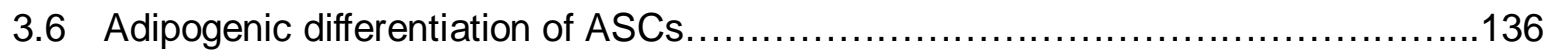

\section{Chapter 4}

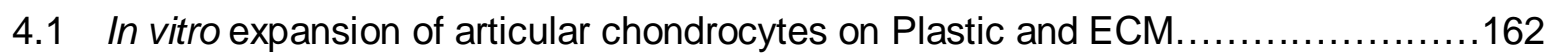

4.2 Immunophenotype changes of chondrocytes expanded on Plastic and ECM.......163

4.3 Redifferentiation capacity of expanded chondrocytes induced by TGF- $\beta 1 \ldots \ldots \ldots . . .164$

4.4 Real-time PCR to evaluate redifferentiation capacity induced by TGF- $\beta 1 \ldots \ldots \ldots . . .165$

4.5 Redifferentiation capacity of expanded chondrocytes induced by serum.............166

4.6 Real-time PCR to evaluate redifferentiation capacity induced by serum .............167

4.S1 Immunohistochemistry of redifferentiated chondrocytes at Day $7 \ldots \ldots \ldots \ldots \ldots \ldots \ldots$. 168

4.S2 Biochemistry and real-time PCR of redifferentiated chondrocytes at Day $7 \ldots \ldots \ldots 109$

\section{Chapter 5}

5.1 Effect of SDSC-derived ECM on NP cell morphology and proliferation...............192

5.2 low cytometry analysis of typical MSC surface antigens in NP cells................193

5.3 Quantitative real-time PCR of dedifferentiated NP cells ......................... 194

5.4 Histological evaluation of redifferentiation capacity of NP cells....................195

5.5 Biochemical analyses of redifferentiation capacity of NP cells $\ldots \ldots \ldots \ldots \ldots \ldots \ldots \ldots \ldots$

5.6 Real-time PCR of redifferentiation capacity of NP cells........................... 197

\section{Chapter 6}

6.1 Extracellular Matrix (ECM) induces changes of the human BMSCs................227

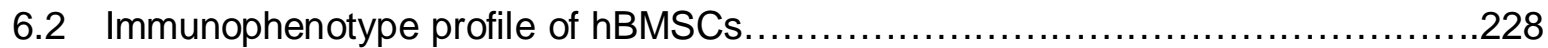

6.3 Immunoblotting of hBMSCs when expanding on Plastic and ECM.................231

6.4 Phase image and immunohistochemistry of chondrogenic hBMSC pellets...........232

6.5 Biochemistry to analyze hBMSCs chondrogenic potential ......................233

6.6 Real-time PCR to analyze hBMSCs chondrogenic potential .....................234

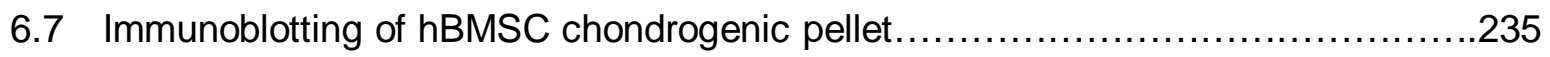

\section{Chapter 7}

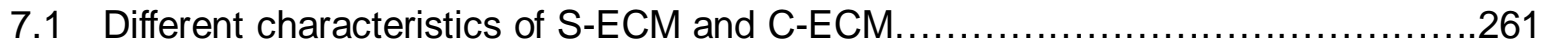

7.2 Immunohistochemistry and biochemistry of chondrogenic SDSC pellets............262

7.3 Real-time PCR to analyze SDSC chondrogenic potential $\ldots \ldots \ldots \ldots \ldots \ldots \ldots \ldots \ldots \ldots \ldots \ldots \ldots \ldots \ldots \ldots$ 


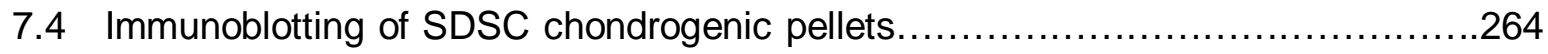

7.5 Immunohistochemistry and biochemistry of chondrocyte pellets..................265

7.6 Real-time PCR to analyze chondrocyte chondrogenic potential ...................266

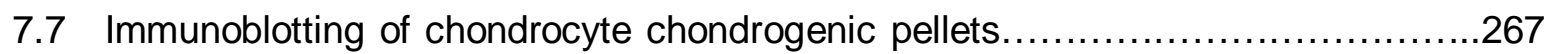




\section{LIST OF TABLES}

\section{Chapter 1}

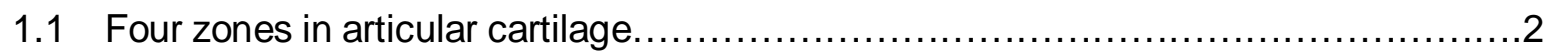

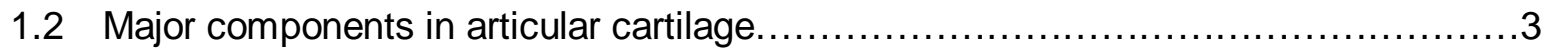

1.3 Biomechanical properties of normal and aged human cartilage $\ldots \ldots \ldots \ldots \ldots \ldots \ldots \ldots \ldots$

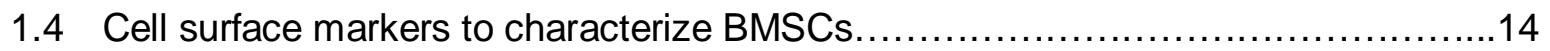

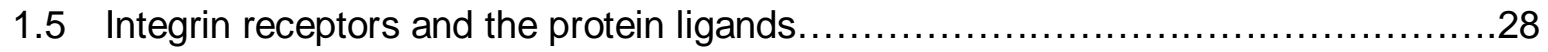

\section{Chapter 2}

2.1. TaqMan ${ }^{\circledR}$ customized porcine marker gene primers and probes.... 103 


\section{SPECIFIC AIMS}

Articular cartilage is a unique, hypocellular and avascular tissue, mostly made of extracellular collagens and proteoglycans (PGs), supporting frictionless movements and loading-bearing capacity of articulation. ${ }^{1}$ Articular cartilage has a limited ability to heal after trauma and cartilage lesions often progress to cartilage degeneration if untreated. Mesenchymal stem cells (MSCs) are considered an attractive source for cell therapy and regenerative medicine because of their excellent self-renewal and differentiation potentials to multiply lineages. ${ }^{2}$ Conventional monolayer expansion system in vitro limits the utilization of MSCs for regeneration of cartilage defects and intervertebral disc, because MSCs rapidly lose their self-renewal ability and multipotent differentiation capacity. ${ }^{3 ; 4}$ The lack of a proper microenvironment in current two-dimensional (2D) expansion system is proposed to be responsible for the loss of multipotential differentiation in MSCs. ${ }^{5}$ We hypothesize that a nature three-dimensional (3D) cell-deposited extracellular matrix (ECM) can provide a proper microenvironment to improve MSC self-renewal and multipotent differentiation.

Natural ECM provides a structural scaffold to resist tensile and compressive stress and functions as a tight connection to cytoskeleton of cells through cell-surface receptors, enabling cells to sense and respond to mechanical and chemical signals. ${ }^{6}$ Compared to traditional 2D ECM, this natural 3D ECM is composed of multiple matrix proteins such as collagens, fibronectin and proteoglycans. ${ }^{7}$ In the structure, 3D ECM is more pliable than 2D ECM, has more fibrillar matrix and contains the space originally occupied by the cells generating matrix. The native cell-deposited ECM not only provides a proper niche to maintain MSC self-renewal and differentiation potential but also establishes a model to mimic the interaction in vivo between cells and $\mathrm{ECM}^{8}$

Our long term goal is to develop a strategy that can maintain MSCs self-renewal and promote the chondrogenic potential in the in vitro culture system, to improve the current therapy of autologous cell transplantation and to investigate the interaction between cells and ECM. The objectives of this dissertation are: (1) compare the effects of 3D ECM and 2D 
conventional plastic culture on MSCs; (2) improve the culture conditions of terminally differentiated cells by adopting 3D ECM; (3) investigate the interaction between MSCs and ECM; (4) compared extracellular matrices deposited by two distinct types of cells. The central hypothesis is that natural cell-deposited ECM serves as an in vitro 3D stem cell niche to maintain self-renewal and enhance the chondrogenic potential.

\section{Specific Aims:}

Specific aim 1: Compare the proliferative rate and chondrogenic potential of MSCs on 3D cell-deposited ECM and the conventional plastic culture.

1.1 Generate and characterize 3D ECM deposited by synovium-derived stem cells (SDSCs).

1.2 Expand SDSCs on 3D ECM and conventional plastic culture and compare the chondrogenic potentials.

1.3 Expand and compare adipose-derived stem cells (ADSCs) on SDSC-deposited ECM, ADSC-deposited ECM and conventional plastic culture.

Hypothesis 1: We hypothesize that 3D ECM will improve SDSCs and ADSCs self-renewal ability and enhance chondrogenic potential. More specifically, we believe that SDSCs and ADSCs expanded on ECM will show a higher proliferative rate than the cells cultured on conventional plastic surface. In a pellet culture system, SDSCs and ADSCs will exhibited a higher chondrogenic gene expression and cartilage-specific proteins and matrix.

\section{Specific aim 2: Use SDSC-deposited ECM to improve in vitro culture of terminally} differentiated cells such as articular chondrocytes and nucleus pulposus cells.

2.1 Expand articular chondrocytes on 3D SDSC-deposited ECM and conventional plastic flasks and investigate the changes of cell proliferative rate, immunophenotype profile and the 
level of dedifferentiation.

2.2 Induce articular chondrocytes expanded on ECM and plastic flasks toward redifferentiation by incubation of TGF- $\beta$-containing medium or serum-containing medium.

2.3 Expand and compare cell proliferative and redifferentiation potentials of nucleus pulposus cells on 3D ECM and conventional plastic flask.

Hypothesis 2: We hypothesize that 3D SDSC-deposited ECM will promote the proliferation of articular chondrocytes and nucleus pulposus cells, delay the process of dedifferentiation and enhance the effect of redifferentiation.

Specific aim 3: Investigate the interaction between ECM and bone marrow stem cells (BMSCs) and the potential mechanism of the enhanced chondrogenic differentiation after expansion on ECM.

3.1 Investigate BMSCs expanded on ECM including proliferative rate, immunophenotype profile, intracellular oxygen reactive species and apoptotic response.

3.2 Explore integrin system and intracellular signaling pathways that affect BMSC self-renewal.

3.3 Induce BMSCs toward chondrogenic differentiation and elucidate the mechanism for the improved chondrogenic potential.

Hypothesis 3: We hypothesize that 3D ECM interacts cells through integrin system to activate Src/ERK signaling pathway. Phosphorylated ERK1/2 will increase the expression and activation of cyclin D1 to promote cell cycle from G1 to S phase. The elevated cyclin D1 will be responsible for the improved cell self-renewal. Meanwhile, 3D ECM will increase the responsiveness of TGF- $\beta$ receptors to enhance chondrogenic differentiation. 
Specific aim 4: Compare two different extracellular matrices separately deposited by SDSCs and articular chondrocytes on the effects of cell proliferation and chondrogenic potential.

4.1 Obtain ECMs from SDSCs and chondrocytes and compare the components in the two types of ECM.

4.2 Expand SDSCs and chondrocytes separately on conventional plastic flaks, SDSC-deposited ECM and chondrocyte-deposited ECM.

4.3 Compare chondrogenic differentiation of SDSCs and chondrocytes expanded under the three different conditions.

Hypothesis 4: We hypothesized that ECM from tissue-specific sources will improve the proliferation and chondrogenic potential differently. More specifically, we believe that SDSCS cultured on SDSC-deposited ECM will have a superior effect on self-renewal and chondrogenesis to those on chondrocyte-deposited ECM. We also believe that chondrocyte cultured on chondrocyte-deposited ECM will have a better result in cell proliferation and redifferentiation than those on SDSC-deposited ECM. 


\section{REFERENCES}

1. Little K. Nature of osteopetrosis. Br.Med.J. 1969;2:49-50.

2. Curl WW, Krome J, Gordon ES et al. Cartilage injuries: a review of 31,516 knee arthroscopies. Arthroscopy 1997;13:456-60.

3. Bonab MM, Alimoghaddam $\mathrm{K}$, Talebian $\mathrm{F}$ et al. Aging of mesenchymal stem cell in vitro. BMC.Cell Biol. 2006;7:14.

4. Minguell JJ, Erices A, Conget P. Mesenchymal stem cells. Exp.Biol.Med.(Maywood.) 2001;226:507-20.

5. Yamada KM, Cukierman E. Modeling tissue morphogenesis and cancer in 3D. Cell 2007;130:601-10.

6. Cukierman E, Pankov R, Stevens DR et al. Taking cell-matrix adhesions to the third dimension. Science 2001;294:1708-12.

7. Cukierman E, Pankov R, Yamada KM. Cell interactions with three-dimensional matrices. Curr. Opin. Cell Biol. 2002;14:633-9.

8. Green JA, Yamada KM. Three-dimensional microenvironments modulate fibroblast signaling responses. Adv.Drug Deliv. Rev. 2007;59:1293-8. 


\section{Chapter 1:}

\section{Literature Review}




\subsection{Articular Cartilage Biology}

Cartilage is a unique tissue in articulated joints covering long bones and provides frictionless movement and a mechanical loading-bearing surface. Cartilage transfers the force generated by underlying bones and supports a large range of joint motion with lubrication and stability. ${ }^{1-3}$ The structure of cartilage can be divided into four zones: superficial zone, middle zone, deep zone and calcified cartilage zone shown in Table 1.1 and Figure 1.1. ${ }^{2-5}$

\begin{tabular}{|c|c|c|}
\hline Zones & Thickness & Composition \\
\hline Superficial zone & $\begin{array}{l}200 \mu \mathrm{m} \\
10-20 \% \text { of the cartilage thickness }\end{array}$ & Tangentially oriented collagen fibers \\
\hline Middle zone & $\begin{array}{c}1 \mathrm{~mm} \\
60 \% \text { of the cartilage thickness }\end{array}$ & Randomly oriented collagen fibers \\
\hline Deep zone & $\begin{array}{l}600 \mu \mathrm{m} \\
30 \% \text { of the cartilage thickness }\end{array}$ & $\begin{array}{l}\text { Perpendicularly oriented collagen } \\
\text { fibers }\end{array}$ \\
\hline Calcified zone & Interface with the bone & $\begin{array}{l}\text { Large bundled collagen fibers } \\
\text { anchored within subchondral bone }\end{array}$ \\
\hline
\end{tabular}

Table 1.1 Four zones in articular cartilage

Cartilage is composed of $1 \%$ chondrocytes and $99 \%$ the matrix. ${ }^{4 ; 6}$ There are two major functions of chondrocytes: 1) to produce sufficient collagens and proteoglycans to form mature cartilage tissue in the developmental stage; and 2) to release enzymes to break down aging components and replace with newly generated matrix. ${ }^{4 ; 6 ; 7}$ The size, morphology and arrangement of chondrocytes vary with the depth of cartilage. From the surface of cartilage to the deeper level, chondrocytes change from flatter to rounder and from a scattered to an organized arrangement. The major compositions of cartilage include water (70\%), type II collagen (15\%) and proteoglycans (15\%). The function of each component in cartilage is demonstrated in Table 1.2 and Figure 1.2..$^{4 ; 6 ; ; 9}$

\begin{tabular}{|l|l|l|}
\hline Component & Percentage & Function \\
\hline
\end{tabular}




\begin{tabular}{|c|c|c|}
\hline Chondrocytes & $1 \%$ & $\begin{array}{c}\text { Produce extracellular matrix and enzymes } \\
\text { to break down aging contents }\end{array}$ \\
\hline Collagen (major Type II) & $15 \%$ & $\begin{array}{c}\text { Provides cartilage with its tensile strength } \\
\text { and stiffness }\end{array}$ \\
\hline Proteoglycans & $15 \%$ & $\begin{array}{c}\text { Provide resiliency to compression and } \\
\text { elasticity }\end{array}$ \\
\hline Water & $70 \%$ & $\begin{array}{c}\text { Provides suitable environment for } \\
\text { chondrocyte survival and matrix function }\end{array}$ \\
\hline
\end{tabular}

Table 1.2 Major components in articular cartilage

Cartilage functions to transfer force and bear loads, and the biomechanical properties of human articular cartilage have been extensively studied. Cartilage provides resiliency not only to tensile loads but also to compressive forces depending on different matrix compositions. Cartilage has a relatively low compressive stiffness ( $E=0.1-2.0 \mathrm{MPa}$ ), Poisson's ratio $(v=0.2)$ and permeability. ${ }^{10 ; 11}$ Table 1.3 shows other biomechanical properties of normal and aged human cartilage; the mechanical properties of cartilage decrease with aging. ${ }^{12 ; 13}$

\begin{tabular}{|c|c|c|}
\hline Mechanical Parameter & Normal Cartilage & Aged Cartilage \\
\hline Young's modulus $(\mathrm{MPa})$ & 122 & 76 \\
\hline Elastic energy $\left(\mathrm{kJ} / \mathrm{m}^{3}\right)$ & 141 & 23 \\
\hline Viscoelastic energy $\left(\mathrm{kJ} / \mathrm{m}^{3}\right)$ & 42.5 & 22.9 \\
\hline
\end{tabular}

Table 1.3 Biomechanical properties of normal and aged human cartilage

\subsection{Cartilage defects and degenerative diseases}

\subsubsection{Three cartilage defects}

Three typical cartilage defects occur in clinical practice, including subchondral, full-thickness and osteochondral defects (Figure 1.3). ${ }^{14}$ Subchondral defect is typically a focal lesion limited to the superficial or middle zone of cartilage. Full-thickness defect extends to subchondral 
bony plate but stays intact. Osteochondral defect includes damage to cartilage and subchondral bone tissue..$^{15-17}$

\subsubsection{Trauma}

Young patients usually suffer cartilage-related diseases caused by trauma. Clinical focal lesions after trauma are usually very small $\left(<1 \mathrm{~cm}^{2}\right)$ and happen only on the articular cartilage surface.$^{14-17}$ Due to the limited self-healing ability of articular cartilage, focal lesions if untreated may progess to degenerative disease. ${ }^{18}$

\subsubsection{Osteoarthritis}

Osteoarthritis (OA) is one of the oldest and most common diseases in human history; it is defined as a degenerative disease of joints affecting not only articular cartilage but also menisci and bone tissue. The symptoms of OA include steady or intermittent pain in joints, stiffness after a period of inactivity such as sleeping and sitting, swelling or tenderness in one or more joints, reduced range of motion and cracking in joints. ${ }^{3}$

Besides trauma, several risk factors may lead to OA. Age is a main risk; it is reported that most people over the age of 60 have OA of some severity and $72 \%$ of OA patients are over 40-years old. ${ }^{3}$ Gender is another factor related to OA; women are more likely to be affected than men. $\mathrm{OA}$ is more common in men before age 45 and in women after age $45 .{ }^{17 ; 19}$ Hereditary gene defects result in damaged collagen components in cartilage and obesity leading to joint overload. ${ }^{17}$ Other risk factors include metabolic problems such as diabetes or alcohol abuse. ${ }^{19}$

Four grades of macroscopic changes in osteoarthritic articular cartilage were proposed by Outerbridge. In grade 1, cartilage becomes soften in some areas. In grade 2, there is fragmentation a half inch or less in diameter. In grade 3, the fragmented area enlarges to more than half an inch, and in grade 4, the injuries develop to full-thickness defects or to subchondral bone tissue (Figure 1.4). ${ }^{20}$ 
Articular cartilage becomes soften, fibrillated, and eroded down to the subchondral bone in OA. At the microscopic level, OA results in chondrocyte cell death and the degradation of cartilage matrix. ${ }^{21 ; 22}$ Since chondrocytes are the only cellular components in articular cartilage, they are able to respond to cartilage damage caused by OA, firstly by clustering and proliferating. Moreover, OA increases the production and activity of cytokines, chemokines and other inflammatory mediators from immune cells, synovial cells and chondrocytes. Inflammatory mediators include interleukins such as IL-1, IL-6, IL-7, IL-17 and IL-18; reactive oxygen species such as hydrogen peroxide, superoxide and nitric oxide; and lipid-derived mediators such as prostaglandins. ${ }^{23-25}$ The mediators induce chondrocyte apoptosis, proteolysis and cell death. In OA, proteolytic enzymes destroy cartilage matrix by cleaving type II collagen without degrading type IX or type XI collagen. ${ }^{21 ; 24}$

Recently the Centers for Disease Control and Prevention (CDC) reported that 27 million Americans suffered from OA and that females were more likely to be affected than males. By the year 2030, $25 \%$ of adult U.S. citizens, nearly 67 million people, will have arthritis and other cartilage degenerative diseases. A study conducted by the researchers in Stony Brook (NY) showed that about $0.8 \%$ of women and $0.3 \%$ of men nationwide had OA. The annual medical care expended on OA is about $\$ 185.5$ billion and work absence due to OA costs employers over $\$ 10$ billion per year in U.S..$^{26 ; 27}$

\subsection{Intervertebral disc degeneration}

Intervertebral discs (IVD) are between two vertebrae in human spine. The total number of discs in spine is twenty-three. IVD composes $20 \%$ to $30 \%$ of the length of spine and the size of discs increases from cervical to lumbar regions. IVD degeneration is the primary cause of low back pain. It is reported that, in US, approximately 5.7 million people suffer with IVD degeneration and most of them are in the 45 to 64 year old range. ${ }^{28-30}$

Intervertebral discs have the following functions: 1) manage the movement of fluid within the nucleus that allows vertebrae to rock back and forth and increases flexibility; 2) serve as shock absorbers; 3 ) act as pads to maintain the space between the 24 movable vertebrae; 4) 
allow extension and flexion. ${ }^{31 ; 32}$

The structure of IVD contains three avascular tissues: annulus fibrosus (AF), nucleus pulposus (NP) and cartilage endplates (Figure 1.5A). ${ }^{33} \mathrm{NP}$, located at the center of IVD, acts as a spongy tissue and is composed of a large amount of water, proteoglycans and collagens to resist compression. Chondrocyte-like cells are found in NP tissue; the cell density is $4 \times$ $10^{6}$ cells $/ \mathrm{cm}^{3}$ at maturity. ${ }^{28 ; 31 ; 32}$ Outside NP is annulus fibrosus that is made of a series of lamellae. The concentric collagen fibers that compose AF tissue orient at various angles and bear the upper body weight due to the swelling effect of NP. Fibroblast-like cells are found in AF with a relatively higher cell density of $9 \times 10^{6} \mathrm{cells} / \mathrm{cm}^{3}$. The cell density in NP and AF is much less compared to that in articular cartilage of $1.4 \times 10^{7}$ cells $/ \mathrm{cm}^{3}$. Cartilage endplates, a thin horizontal layer, are on the superior and inferior faces of IVD and function to regulate the diffusion of nutrients. ${ }^{28-30}$

In normal IVD, the compositions of AF and NP include a network of collagens including type I, II, III, V, VI, IX, XI, XII, and XIV, a variety of proteoglycans that mainly contains aggrecan, versican, decorin, biglycan and fibromodulin, and other matrix proteins such as fibronectin and elastin. ${ }^{28-30}$ However, the proportion of matrix components varies from NP to AF and the inner to the outer part of AF. The outermost layers of AF are composed of mostly type I collagen and NP contains type II collagen. From the outer to the inner AF, type I collagen decreases and type II collagen increases. Type $X$ collagen is also found in NP and inner AF layers. Aggrecan is a major component of matrix proteoglycans in AF and NP. In NP the proportion of aggrecan and water content is about $50 \%$ to $80 \%$ of the wet weight but, in $\mathrm{AF}$, it only holds $20 \%$ to $70 \%$ from the outer to inner part. ${ }^{29-31 ; 34}$

IVD degeneration progresses with age or after trauma due to the poor self-repair mechanism similar to articular cartilage. Loss of proteoglycans is the most significant change in IVD degeneration and aggrecan degrades from large molecules to small fragments (Figure 1.5B). ${ }^{30}$ The degeneration progresses steadily in NP since aggrecan composes most of the matrix and the loss of aggrecan results in dysfunction in loading-bearing and back pain. ${ }^{29-31}$ 
The network of collagen matrix also undergoes degeneration but not as obvious as that of proteoglycans. The types and distribution of collagens alter in NP during IVD degeneration; type II collagen becomes denatured and more type I collagen is synthesized. Except the significant decrease in matrix components, some enzyme production and activity increases, such as matrix metalloproteinases (MMPs), cathepsins and aggrecanases. ${ }^{28-32}$ The activity of these enzymes is higher in degenerated IVD than in normal IVD to enhance further matrix degradation. ${ }^{34-36}$

During the process of IVD degeneration, NP cells are stimulated to proliferate and the cell density increases. ${ }^{36 ; 37}$ However, the level of cell death and cell senescence increases simultaneously. Shortening telomeres when cells undergo repeated division and an increased generation of reactive oxygen species and cytokines would be the reason for cell death and senescence. $^{38-40}$ The phenotype of NP cells in IVD degeneration also changes caused by synthesizing different matrix proteins from NP cells, secretion of growth factors, an increase in the level of inflammatory factors and enhanced enzymatic activity. ${ }^{35 ; 41}$

\subsection{Current therapy tragedies for articular cartilage repair and IVD degeneration}

\subsubsection{Intrinsic self-repair of articular cartilage}

Articular cartilage has very limited self-healing ability once trauma or degeneration occurs because the avascular structure prevents mesenchymal stem cells in bone marrow or chondrocyte progenitors from migrating to damaged areas. Some animal studies showed that spontaneous healing was observed when minor lesions were on the surface of cartilage or subchondral defects were less than $3 \mathrm{~mm}$ in diameter. ${ }^{17 ; 19}$ However, articular cartilage fails to repair the large defects that extend to osteochondral tissue and the damages ultimately lead to cartilage degeneration. The most important issue in cartilage self-repair is that the repaired chondral tissue is fibrocartilagenous in which the ratio of type I collagen is much higher than type II collagen with fewer proteoglycans. This fibrocartilagenous tissue fails to have normal biomechanical functions or withstand load-bearing like hyaline cartilage tissue. Eventually the fibrocartilagenous tissue leads to degradation and loss of cartilage tissue. ${ }^{19 ; 22 ; 42 ; 43}$ 


\subsubsection{Conservative treatments and surgical procedures}

Steroidal and non-steroid anti-inflammatory drugs are commonly used to treat cartilage defects but those medicines are mainly for pain relief. Hyaluronic acid can be injected into the joints to reduce pain. At present D-glucosaminesulphate and chondroitinsulphate are proven to improve articular cartilage healing. Overall, the effectiveness of drug treatments is minor and none of them can fully heal cartilage defects. ${ }^{19 ; 42}$

Surgical procedures are used to stimulate the intrinsic repair in articular cartilage by drilling or microfracturing subchondral bones. The purpose of these procedures is to release the mesenchymal stem cells in blood and bone marrow to recruit into the defects. However, fibrocartilagenous or scar tissue is formed and therefore is susceptible to degradation. ${ }^{14 ; 19 ; 42 ; 44}$

Transplantation of periosteum and perichondrium was used in cartilage repair; the tissue covered articular cartilage defects. The procedure provided a fast reconstruction of the cartilage surface but resulted in calcification and eventually degeneration in the defect area. The transplantation of pure periosteum and perichondrium is no longer recommended in clinical use..$^{14 ; 19 ; 45}$

Osteochondral transplantation is the procedure in which osteochondral plugs are harvested from less used load-bearing areas in joints and transferred into cartilage defects. The technique can be used to repair large defects and provides rapid bone healing to fix the osteochondral plugs into the implant site. The disadvantages of the procedure include donor site morbidity, increased cell death of chondrocytes and formation of fibrocartilagenous tissue which leads to further cartilage degeneration. 8;14;46-49

\subsubsection{Autologous chondrocyte transplantation}

Autologous chondrocyte transplantation (ACT) has been used in clinical practice since 1994 and has been proven more effective than other surgical procedures..$^{50}$ In the first step of ACT, a small amount of cartilage tissue is taken by arthroscopy from a non-weight bearing area 
(150 - $300 \mathrm{mg}$ ). In the second step, chondrocytes are released from biopsy by enzymatic digestion and expanded in vitro to reach an adequate cell number for intra-articular injections. In the third step, cultivated chondrocytes are injected into articular cartilage damage sites, covered with a periosteal graft and sutured to prevent water leak (Figure 1.6). ${ }^{43 ; 43 ; 50 ; 51}$

The first generation of ACT relied on cultured chondrocytes and injecting cells beneath a periosteal graft. It has several disadvantages including surgical damage in cartilage biopsy, cell-phenotype change in the process of chondrocyte expansion in vitro (also called dedifferentiation), leakage of chondrocytes from the defects and periosteal complications such as hypertrophy and detachment from the injection site. $8: 42 ; 43 ; 51$

The second generation of ACT uses a bi-layer collagen membrane instead of periosteal graft. The collagen membrane is sutured onto the cartilage defects and expanded chondrocytes are injected underneath. The replacement of collagen membrane to periosteal graft simplifies the surgical procedure, reduces the risk in periosteal surgery and decreases the number of incisions in patients. ${ }^{8 ; 42 ; 43 ; 51}$

The third generation of ACT is also called the "all-in-one" procedure; it uses three-dimensional biocompatible scaffolds where chondrocytes are seeded and differentiate to generate cartilage-specific matrix. The newly generated cartilage repair graft is designed to fit the size of defects and fibrin glue is used as sealant to fix the graft. $8 ; 42 ; 43 ; 51$

The clinical outcomes of ACT are successful because hyaline-like cartilage tissues are observed and reach $90 \%$ of native cartilage stiffness. Different from fibrocartilagenous tissues, the hyaline repaired cartilage does not lead to further degeneration and have normal biomechanical functions. ${ }^{14 ; 42 ; 43 ; 51 ; 52}$

However, the disadvantages of ACT are obvious including the limited expandability of chondrocytes and the rapid dedifferentiation in the process of in vitro expansion over several passages. In dedifferentiation, chondrocytes lose their round morphology and change to a 
spindle-shaped, fibroblast-like phenotype. Type I collagen is synthesized in dedifferentiated chondrocytes instead of type II collagen and aggrecan. Another common complication of ACT is periosteal hypertrophy that results in pain in the repaired joints and detachment of periosteal graft leading to the failure of ACT. The ACT procedure includes cartilage biopsy from the patients' healthy joints that might cause surgical defects in cartilage even it is not load-bearing. ${ }^{42 ; 43 ; 51 ; 53-56}$

\subsubsection{Autologous or allogeneic cell transplantation for nucleus pulposus repair}

Mature nucleus pulposus (NP) contains chondrocyte-like cells with a low cell density and limited self-healing. The NP environment is harsh because of high stress, low oxygen tension, low $\mathrm{pH}$ and limited nutrients due to an avascular structure. Intervertebral discs (IVD) face the risk of degeneration with aging and, due to the limited self-repair, the degeneration process is irreversible. $^{57}$

Similar to autologous chondrocyte transplantation (ACT), autologous or allogeneic NP cell transplantation has been used in animal studies. NP cells are first obtained from a healthy IVD and expanded in vitro to reach an adequate cell number, then injected into the degenerated IVD. The injected NP cells are able to synthesize new matrix contents such as aggrecan and type II collagen. The implanted NP cells effectively improve disc conditions without a major immune response. ${ }^{58}$

Worse than articular cartilage, the cell density is much lower in NP and NP cells have less expandability than chondrocytes. It is reported that the cell viability was improved when NP cells were co-cultured with AF cells. ${ }^{59}$ A new co-culture system with MSCs was adopted to increase NP cells' proliferative ability. ${ }^{60}$ Growth factors, such as transforming growth factor $\beta$ and insulin-like growth factor, were supplemented to stimulate NP cell proliferation. ${ }^{60}$ However, the poor expandability of NP cells limits the application of autologous or allogeneic cell transplantation to repair IVD degeneration.

Because the matrix components in articular cartilage are similar to those in IVD, 
transplantation of articular chondrocytes into degenerative IVD was tested and this method effectively reduced the loss of proteoglycans in the injured IVD. ${ }^{57: 61}$ Even though aggrecan and type II collagen are found both in articular cartilage and NP tissue, the ratio of aggrecan to collagen is different. In NP, the ratio of aggrecan to collagen is significantly higher (approximately $27: 1$ ) than that in articular cartilage $(2: 1),{ }^{62}$ indicating that chondrocyte transplantation for IVD is still risky.

\subsection{Adult mesenchymal stem cells}

\subsubsection{Embryonic stem cells and induced pluripotent stem cells}

Embryonic stem cells (ESCs) are identified by the properties of unlimited self-renewal, immortality and pluripotent differentiation to multiple tissues and organs. The study of ESCs began in the 1950s and the culturing system was adopted in 1981 using a fibroblast feeder layer and serum. ${ }^{63 ; 64}$ Thomson et al. in 1998 first created several human embryonic stem cell lines (hESCs). ${ }^{65}$ Human embryonic stem cells are typically isolated from the inner cell mass of the blastocytes and expanded on a feeder cell layer to maintain hESC in an undifferentiated state. After removing hESCs from the feeder cell layer, they are cultured in suspension where embryoid bodies containing differentiated and undifferentiated cell aggregate. Embryoid bodies can be cultured on monolayer later and spontaneously differentiate to three germ lineages. Theoretically, ESCs can differentiate into any desired lineage through stimulation of specific growth factors or under proper protocols. ${ }^{66-69}$

Levenberg et al. ${ }^{70}$ cultured hESCs on a three-dimensional (3D) scaffold to promote cell proliferation and differentiation. A 3D vessel-like structure was observed, characterized by neural tissue, cartilage and liver. ${ }^{70}$ ESCs are a potential candidate for cartilage and bone tissue repair. Jukes et al. developed cartilage tissue-engineered constructs using mouse ESCs on a 3D scaffold in vitro and, after transplantation in vivo, the cartilage construct was ossificated and ultimately replaced by bone tissue. ${ }^{71}$ However, the disadvantages of using hESCs are obvious. The use of hESCs involves the destruction of in vitro human fertilized eggs to obtain blastocytes, which is unacceptable by some people in certain religions and 
countries. A major immune rejection or response can occur when hESCs are implanted into patients. The risk of hESCs forming tumor tissue in vivo is still large. ${ }^{72-76}$

Recently the generation of induced pluripotent stem (iPS) cells provides a novel method and potentially exciting approach for stem cell research, drug testing and tissue engineering. iPS cells are the pluripotent stem cells transferred from terminally differentiated adult somatic cells by induction of several key transcription factors. Takahashi et al. in 2006 successfully developed the first iPS mouse cells by inserting four transcription factors [Sox2, Oct-3, c-Myc and Klf4 (Krueppel-like factor 4)] to reprogram fibroblasts to ES-like cells. ${ }^{77} \mathrm{Yu}$ et al. also

reprogrammed iPS cells using Oct3-4, Sox2, Nanog and Lin28 by lentivirus transfection ${ }^{78}$. In 2008 iPS cells were generated by using adenovirus ${ }^{79 ; 80}$ and, in 2009, the first iPS cells were reported using only recombinant proteins. ${ }^{81-83}$ The use of adenovirus and recombinant proteins can significantly reduce the risk of tumor formation since they do not affect the targeted host genes. Although iPS cells overcome some disadvantages of hESCs, there are still some drawbacks to limit their use. The reprogramming of iPS cells involves viral integration, even though recombinant proteins are in use but with low efficiency. The generation of iPS cells faces the disadvantages of low efficiency and high cost, because the typical efficiency of iPS cell generation is less than 1\%; the efficiency can increase to $28 \%$ if using hematopoietic stem cells or mesenchymal stem cells. ${ }^{66 ; 82 ; 84}$

\subsubsection{Adult stem cells}

A well-known adult stem cell is hematopoietic stem cell (HSC) that can be collected from bone marrow and umbilical cord blood. Hematopoietic stem cells (HSCs) are characterized by cell surface markers, which are positive for CD34, CD133, CD177, CD59 and CXCR4, but negative for lineage-specific antigen, CD38, and CD73 etc. The ability of HSC differentiation into non-hematopoietic lineage tissues is possibly artifact, because mesenchymal stem cells locate or migrate into hematopoietic organs. ${ }^{85-89}$

Mesenchymal stem cells (MSCs) are another type of adult stem cells, characterized by a strong self-renewal ability and multipotent capacity to differentiate into multiple lineages, 
including bone, cartilage, adipose tissue, muscle, tendon, stromal cells and connective tissue. MSCs are characterized by surface markers positive for CD73, CD90 and CD105, but negative for CD45, DC34, CD14, CD11b, CD79a, CD19 or HLA-DR. Three typical MSCs, which are frequently studied in stem cell research and cartilage tissue engineering, will be introduced, including bone marrow stem cells, synovium-derived stem cells and adipose-derived stem cells. ${ }^{73 ; 90-92}$

Ideal MSCs for regenerative medicine include the following criteria: 1) they should have a large cell number for clinical therapy; 2) they should have a minimally invasive procedure to collect or harvest tissue for MSCs; 3) they should have multiple differentiation potentials; 4) they should have safe transplantation for autologous and allogeneic MSCs to avoid immune rejection. ${ }^{93}$

MSCs provide a great potential for regenerative medicine and cartilage tissue engineering. MSCs have several advantages over ESCs and iPS cells. Even though MSCs have a weaker proliferative rate than ESCs, they can be collected from various tissue sources and still provide adequate cells for therapy if culture conditions and protocols are improved ${ }^{94 ; 95}$. The multipotential differentiation to multiple lineage tissues of MSCs can benefit bone and cartilage tissue regeneration, tendon and skeletal muscle repair and cardiac tissue engineering. ${ }^{19 ; 96 ; 97}$ MSCs can be collected directly from patients to avoid any immune rejection. Meanwhile, the results of various immunoassays on MSCs suggest that MSCs have a strong immunosuppressive effect by inhibiting T-cell recognition and suppressing the production of TNF- $\alpha$ or INF- $y$. The immunosuppressive effect enables the use of allogeneic MSCs with minimal immune reaction. ${ }^{91 ; 98-100}$ MSCs are not subject to the ethical dilemmas and forming teratocarcinoma that are associated with ESCs. ${ }^{101-104}$

\subsubsection{Bone marrow stem cells}

Bone marrow stem cells (BMSCs) were first reported by Friedenstein et al. ${ }^{91 ; 105-107}$ The fibroblast-like cells in bone marrow were found through attachment to tissue culture plates and the cells exhibited osteogenic differentiation ability. Since their discovery, BMSCs have 
been studied in depth and found to differentiate into not only osteoblasts ${ }^{108}$ but also chondrocytes, ${ }^{109}$ adipocytes, ${ }^{110}$ myoblasts ${ }^{111}$ and early progenitors of neural cells. ${ }^{112}$

The BMSC population in bone marrow is scant and represents $0.001 \%$ to $0.01 \%$ of the nucleated cells, which is only $1 / 10$ of the proportion of HSCs. BMSCs, however, show a strong proliferation ability in monolayer expansion in vitro. ${ }^{91 ; 107 ; 113}$ The expansion conditions were improved to increase BMSC cell yield and to maintain their differentiation potentials. In the process of in vitro expansion, BMSCs with different cell morphologies are harvested, including thin and spindle-shaped cells and wider cells. It was found that small, thin BMSCs self-renewed rapidly and had a greater potential for multipotential differentiation. In repeated passages, low seeding density can help BMSCs increase their proliferative rate and maintain their differentiation capacity. ${ }^{114-116}$

The immunophenotype profile of BMSCs is similar to other mesenchymal stem cells. BMSCs are positive for CD105, CD73 and CD90 but negative for CD34, CD 45 CD11a CD19 and HLA-DR. Table 1.4 lists the cell surface markers to characterize BMSCs and other MSCs. ${ }^{91 ; 103 ; 117 ; 118}$

\begin{tabular}{|l|l|}
\hline \multicolumn{1}{|c|}{ Positive markers } & \multicolumn{1}{|c|}{ Negative markers } \\
\hline CD9. CD10. CD13, CD29, CD44, CD49a, & CD45, CD34, CD14, CD11A, CD19, CD86; \\
CD49c, CD49e, CD51, CD54, CD58, CD61, & CD80/CD40, CD15, CD18, CD25, CD31, \\
CD71, CD73, CD90, CD102, CD104, CD105, & CD50, CD62E, CD62P, CD117 \\
CD106, CD119, CD120a, CD120b, CD121, & \\
CD127, CD140a, CD166, CCR1, CCR4, & \\
CCR7, CXCR5, CCR10, VCAM-1, CD166, & \\
AL-CAM, ICAM-1, STRO-1, CD340, SSEA-4. & \\
W8B2, W3D5, W4A5, W5C4, W5C5, W7C6, & \\
9A3, 58B1, HEK-3D6 & \\
\hline
\end{tabular}

Table 1.4 Cell surface markers to characterize BMSCs 
BMSCs have been successfully used in animal studies and clinical practices. The injection of BMSCs was used to repair fresh bone fractures and osteochondral defects. The injected cells shortened healing period, enhanced the repair effect in bone fracture treatment, and reduced the chance of future bone fracture. ${ }^{119 ; 120}$ BMSCs were used to repair full-thickness defects in articular cartilage using various scaffolds. The repair effectiveness of BMSC transplantation was better than those not treated with BMSCs. ${ }^{102 ; 121 ; 122}$ However, BMSC injection therapy failed to significantly improve osteoarthritis possibly due to the serious inflammatory reaction caused by arthritis. ${ }^{123}$ Since BMSCs can differentiate to skeletal, smooth and cardiac muscle cells, cardiac regeneration is the focus of current research. In animal studies, autologous BMSCs were used to treat myocardial infarction and improved cardiac function and angiogenesis, ${ }^{96 ; 124 ; 125}$ indicating that BMSC can be useful in cardiomyocyte regeneration.

\subsubsection{Adipose-derived stem cells}

Adipose-derived stem cells (ADSCs), termed "pre-adipocytes" historically, are another promising candidate for regenerative medicine. ${ }^{126 ; 127}$ ADSCs can be digested enzymatically from adipose tissue and separated from adipocytes by centrifugation. ADSCs share similarities with BMSCs such as an extensive proliferative ability and multipotential differentiation capacity to osteoblasts, adipocytes, chondrocytes, myoblasts and neural cells. ${ }^{128-132}$ Compared to BMSCs, ADSCs have several advantages including easy accessibility, abundant adipose tissue source, higher yield of cell numbers and superior proliferative rate. It is reported that approximately 400,000 liposuction surgeries are preformed in U.S. every year and yield about $100 \mathrm{~mL}$ to $3 \mathrm{~L}$ of lipoaspirate tissue that is discarded. ADSCs can be collected safely and conveniently from those abundant lipoaspirate tissues. ${ }^{132-134}$ Surgery risk is reduced by using autologous ADSCs because fat tissue is more easily accessible than bone marrow. Furthermore, $5 \times 10^{3}$ cells can be collected from $1 \mathrm{~g}$ of adipose tissue; that is 500 -fold greater than the number of BMSCs from the same weight of bone marrow. ${ }^{135-137}$ Adipose tissue is considered a rich and safe source for ADSCs.

The cell surface marker expression of ADSCs is similar to that of BMSCs, positive for MSC markers and negative for HSC markers. ${ }^{138}$ Particularly, CD49d is positive and CD106 is 
negative in ADSCs. ADSCs also show increased expression of CD54 (ICAM-1) compared to BMSCs. ${ }^{138-140}$ The proliferative rate of ADSCs in vitro expansion is not different from that of BMSCs, ${ }^{140}$ but the colony forming rate of ADSCs is higher (50 - 100 times more) than BMSCs. ${ }^{141}$

It is not surprising that ADSCs exhibit excellent adipogenic potential when induced in adipogenic differentiation medium. ${ }^{128 ; 138 ; 142}$ Under osteogenic stimulation, ADSCs are able to express osteoblast-specific genes and bone tissue matrix that are compatible with BMSCs. ${ }^{138 ; 142-144}$ In a murine calvarial defect model, ADSCs were transplanted to repair bone defects and the newly generated bone tissue had biological and biomechanical functions. ${ }^{145}$ In chondrogenic differentiation, ADSCs are able to express chondrogenic-specific genes such as Sox9, aggrecan and type II collagen and generate cartilage-related extracellular matrix of sulfated glycosaminoglycans and type I and II collagens. ${ }^{138 ; 142 ; 146}$ However, compared to BMSCs, ADSCs showed no difference in chondrogenic ability in two-dimensional culture but were much weaker in a three-dimensional system. ${ }^{147}$

\subsubsection{Synovium-derived stem cells}

Synovium-derived stem cells (SDSCs) are from "synovial fibroblasts" that are considered tissue-specific cells for regeneration medicine and cartilage repair. ${ }^{92 ; 148 ; 149}$ Compared to BMSCs, ADSCs and other MSCs, synovial membrane is located near articular cartilage and provides suitable MSCs to migrate into subchondral defects. SDSCs have several advantages over BMSCs and ADSCs including higher proliferative rate, regardless of donor age, and a stronger chondrogenic potential with minimal hypertrophy risk.

Synovial membrane can be harvested by arthroscopy with minimal invasiveness and less risk to donors because of its strong regenerative capacity. SDSCs can be released by enzyme digestion. Approximately 21,000 cells can be harvested from $1 \mathrm{mg}$ of synovium tissue which provides a sufficient cell number for animal studies and clinical practices. ${ }^{92}$ The expandability of SDSCs is identical to BMSCs and superior to ADSCs; SDSCs can be expanded over 10 passages without cell senescence (cell morphology changes to flattened and larger and then 
the cells stop proliferating). When seeding at a low cell density, SDSCs show a more rapid proliferative rate than other MSCs. ${ }^{150 ; 151}$ The colony forming ability of SDSC is about 100-fold greater than that of BMSCs and comparable to ADSCs. ${ }^{141}$ With regard to adipogenesis and osteogenesis, SDSCs are identical to BMSCs and ADSCs. ${ }^{140 ; 141 ; 152}$ SDSCs exhibited superior chondrogenic potential compared to BMSCs and ADSCs. With the induction of transforming growth factor- $\beta 1$ (TGF- $\beta 1$ ), both BMSCs and SDSCs generated cartilage-specific genes and matrix that were stronger than ADSCs. ${ }^{152}$ Using the combination of transforming growth

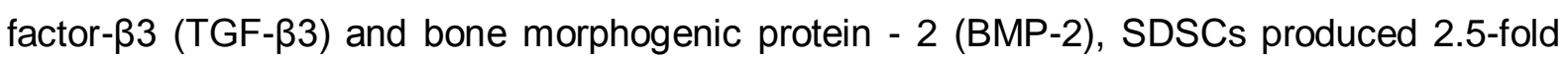
more sulfated glycosaminoglycans and 50\% more collagen than BMSCs. ${ }^{150}$ The superior chondrogenic capacity of SDSCs was also confirmed by other studies. ${ }^{153 ; 154}$

There are two major cell types in synovial tissue, where the round nucleated cells are called "macrophage-like" or "type A" cells and the spindle type cells are named "fibroblast-like" or "type B" cells. Our laboratory developed a "negative isolation" procedure to improve SDSCs' proliferative and chondrogenic capacities. Cells from digested synovial tissue are suspended and incubated with Dynabeads ${ }^{\circledR}$ M-450 conjugated with CD14 antibody specific for macrophages; then the conjugated cells are removed by using the Dynal Magnetic Particle Concentrator $^{\circledR}$ and unconjugated cells are presumed "type B" cells (SDSCs). Figure 1.7 shows the negative isolation procedure in synovial cells. ${ }^{155}$ The negatively isolated SDSCS show improved proliferation and a higher level of matrix generation than untreated synovial cells. ${ }^{149 ; 155}$

\subsection{Chondrogenesis of mesenchymal stem cells}

MSC chondrogenesis is the process where multipotent MSCs differentiate into articular chondrocytes and generate cartilage-specific extracellular matrix, induced by growth factors and nonproteinaceous chemical molecules, in a serum-free medium and under optimal culture conditions such as hypoxia. In the process of chondrogenesis, MSCs continue to express type I and II collagen and aggrecan (chondrogenic markers), and type X collagen (hypertrophic marker) in the late stage. ${ }^{109 ; 149 ; 156-158}$ The first chondrogenesis of MSCs was reported by Ashton et al. ${ }^{156}$ and a pellet culture system was developed by Johnstone et al. to 
enhance chondrogenic effect. ${ }^{109}$ The pellet culture system is widely used to evaluate chondrogenic potential of MSCs in vitro. ${ }^{109 ; 149 ; 157 ; 159}$ The following introduction will focus on growth factors, optimal chondrogenic media and culture conditions and the hypertrophic markers of chondrogenesis. Chondrogenic differentiation signaling pathways and transcription factors induced by TGF- $\beta$ growth factor will be discussed.

\subsubsection{Growth factors in chondrogenesis}

The transforming growth factor- $\beta$ (TGF- $\beta$ ) superfamily is the most commonly used to induce chondrogenesis of MSCs; the family includes TGF- $\beta$, bone morphogenetic proteins (BMPs), activins and inhibins. ${ }^{160}$

TGF- $\beta 1,2$, and 3 promote the synthesis of proteoglycans and type II collagen in MSCs in vitro. $^{159 ; 161}$ TGF- $\beta 1$ in vivo stimulates skeletal tissue development and induces MSC chondrogenic differentiation to repair cartilage defects. ${ }^{19 ; 159 ; 162}$ TGF- $\beta 3$ is proposed to have a stronger effect in MSC chondrogenesis than TGF- $\beta 1$, but our study using SDSCs suggested that there was no difference in chondrogenic effect between these two TGF- $\beta$ factors. From histology and immunohistochemistry, both TGF- $\beta 1$ and TGF- $\beta 3$ can induce SDSCs to generate a large amount of sulfated GAGs and type II collagen. The quantification of chondrogenic index induced by TGF- $\beta 1$ and TGF- $\beta 3$ shows no statistical difference. (Figure 1.8)

Bone morphogenetic proteins (BMPs) are important members of the TGF- $\beta$ superfamily that contain a sub-class of 20 polypeptides. Several BMPs can promote MSC chondrogenesis and cartilage formation by increasing the expression of aggrecan and collagens, such as BMP-2, -4, -6, -7, -13 and -14. Interestingly, BMP-3 inhibits MSC chondrogenesis and osteogenesis by inhibiting BMP-2 activity. ${ }^{163 ; 164}$ The combination of TGF- $\beta$ and BMPs can enhance MSC chondrogenic differentiation, proven by previous studies ${ }^{165}$ and our research on ADSCs (Chapter 3).

The insulin-like growth factor (IGF) family including IGF-I and IGF-II is also investigated in 
MSC chondrogenic differentiation. The binding of IGF to receptors induces activation of different intracellular downstreams and subcutaneously enhances the expression of chondrogenic genes and proteins. ${ }^{159 ; 166}$ In articular cartilage, IGF-I is produced to maintain chondrocyte morphology and induce cell migration to repair defects. It has been proven that IGF-I plays an important role in embryonic development and that the mutation of IGF-I results in serious developmental defects in various musculoskeletal tissues. ${ }^{159 ; 166 ; 167}$

The fibroblast growth factor (FGF) family is also used to improve MSC chondrogenesis and includes 22 sub-class proteins binding to receptors. ${ }^{168}$ The intracellular signaling pathway of FGF is characterized to Ras-MAPK (mitogen activated protein kinase) pathway. ${ }^{169}$ The most well-known member of the FGF family is FGF-2, which functions to promote the proliferation of MSCs and chondrocytes and to enhance chondrogenic differentiation with other TGF- $\beta$ factors. ${ }^{170}$ The knock-out FGF $^{-1-}$ mice had serious issues in cartilage and bone development. ${ }^{159 ; 168}$ In the adult rabbit model, the induction of FGF-2 improved the repair of cartilage defects. ${ }^{171}$

\subsubsection{Optimal chondrogenic conditions}

In addition to protein-based growth factors, several nonproteinaceous chemical compounds are used as supplements in the induction of MSC in vitro chondrogenesis. Dexamethasone is a synthetic glucocorticoid that can enhance MSC chondrogenesis. The normal concentration of dexamethasone is $10^{-7} \mathrm{M}$ and the combination with TGF- $\beta$ can induce a greater generation of aggrecan and type II collagen. ${ }^{109 ; 172}$ Ascorbic acid (vitamin C) is demonstrated to have a stimulatory effect on chondrogenic differentiation in adult MSCs and in embryonic limb development. ${ }^{173 ; 174}$ Insulin is also added to promote in vitro chondrogenesis of MSCs and chondrocytes and is used at a higher level than that in normal human serum. ${ }^{175 ; 176}$

Hypoxia, a low oxygen tension (1\% - 5\%), can improve MSC chondrogenesis demonstrated by several studies. The improvement of chondrogenic differentiation in hypoxia is related to an elevated expression of hypoxia-inducible factors (HIFs), specifically HIF-1a, and chondrogenic transcription factor Sox-9. Thus, protein downstream affects MSCs to generate 
an increased level of cartilage-specific matrix. ${ }^{177-180}$ Hypoxia is also demonstrated to inhibit the formation of fibrous cartilage by decreasing the marker genes of type I and III collagen, ${ }^{181}$ and to induce dedifferentiated chondrocytes in primary culture towards redifferentiation. ${ }^{182 ; 183}$

\subsubsection{Hypertrophy}

Hypertrophy is the terminal stage in chondrogenesis where ossification occurs in cartilage matrix and chondrocytes change their morphology to fibroblast-like cells and eventually differentiate into osteoblasts and form endochondral bone tissue. Typical hypertrophic markers include type $X$ collagen, matrix metalloproteinase 13 (MMP13), alkaline phosphatase (ALP), bone sialoprotein type II (IBSP), secreted phosphoprotein type 1 (SPP1, or osteopontin) and Runt domain transcription factor (Runx2). ${ }^{184 ; 185}$ The fate of hypertrophic chondrocytes is either to differentiate to osteoblasts or to undergo apoptosis, and this will jeopardize the effect of MSC-generated cartilage tissue. ${ }^{186 ; 187}$

BMP-2 and BMP-4 not only stimulate MSCs toward chondrogenesis but also induce the expression of hypertrophic marker genes. ${ }^{188}$ However, TGF- $\beta$ can suppress hypertrophy in chondrocytes. ${ }^{189}$ Intracellular reactive oxygen species (ROS) play an important role in chondrogenesis and hypertrophy. An elevated level of ROS is found in hypertrophic chondrocytes and antioxidant treatment can reverse the excessive hypertrophy. Morita et al. demonstrated that increased ROS can inhibit proliferation and initiate hypertrophic changes in chondrocytes. ${ }^{187}$ Type $X$ collagen is the only well-accepted marker for chondrocyte hypertrophy that is regulated by Runx2, a transcription factor in osteogenesis, and the suppression of Runx2 in chondrogenic differentiation inhibit the expression of hypertrophic genes. ${ }^{190}$

Hypertrophy is one of the major concerns in MSC-derived cartilage tissue engineering, not only ossification but also vascularization. Articular chondrocytes show no signs of hypertrophy including negative expression of type $X$ collagen and resistance to calcification, but MSC-derived chondrocytes have the potential to mineralize and develop vascular invasion after transplantation in vivo. ${ }^{184 ; 185}$ Parathyroid hormone-like peptide (PTHrP) is 
proposed as an inhibitor to hypertrophy in in vitro MSC chondrogenesis. ${ }^{191}$ A recent study showed that the coculture of articular chondrocytes and MSCs can significantly suppress MSC hypertrophy during chondrogenesis because of PTHrP secreted by articular chondrocytes. ${ }^{192}$ In future, the mechanism of hypertrophy in MSC chondrogenesis needs to be studied to improve cartilage tissue engineering.

\subsubsection{Chondrogenesis of SDSC induced by the combination of TGF- $\beta 1$, IGF-I and FGF-2}

Our previous study investigated SDSC chondrogenesis and improved chondrogenic differentiation by growth factor cocktail: TGF- $\beta 1$, IGF-I and FGF-2. ${ }^{149}$

Following negative isolation, SDSCs were centrifuged into pellets and cultured in serum-free chondrogenic medium. The SDSC pellets were divided into five groups. All five groups were treated with $50 \mathrm{ng} / \mathrm{mL}$ of FGF-2 for three days and then induced to chondrogenesis by the growth factor cocktail (10 ng/mL of TGF- $\beta 1$ and $500 \mathrm{ng} / \mathrm{mL}$ of IGF-I) for 15 days. The detail experiment schedule is shown in Figure 1.9A. Group D1 was treated only with $10 \mathrm{ng} / \mathrm{mL}$ of TGF- $\beta 1$. Group D2 was treated with $10 \mathrm{ng} / \mathrm{mL}$ of TGF- $\beta 1$ for 11 days and switched to the cocktail for 4 days. Group D3 was treated with TGF- $\beta 1$ for 7 days and the cocktail for 8 days. Group D4 was treated with TGF- $\beta 1$ for 4 days and the cocktail for 11 days. Group D5 was treated with the cocktail for 15 days (Figure 1.9A).

Histology and immunohistochemistry showed that, with the cocktail treatment of TGF- $\beta 1$ and IGF-I, SDSC pellets had the strongest potential of chondrogenesis. Group D5 had the extensive expression of sulfated GAGs and type II collagen. Type I collagen was positive in all the groups but type $X$ collagen was negative (Figure 1.9B). Biochemistry and real-time PCR also showed that the combination of TGF- $\beta 1$ and IGF-I can maximally induce the expression of chondrogenic matrix and genes. This study suggested a new strategy to induce chondrogenesis of MSCs by the sequential combination of growth factors.

\subsubsection{Intracellular signaling pathways of TGF- $\beta$ induced chondrogenesis}

Members of the TGF- $\beta$ superfamily bind to type II and type I TGF- $\beta$ receptors. Both type II 
and type I receptors contain serine/threonine kinase domains in the intracellular parts. In the process of binding TGF- $\beta$ to type II receptor, type I receptor is recruited and phosphorylated. Following the phosphorylation, type I receptor phosphorylates and activates intracellular signaling pathways, including Smad, mitogen-activated protein kinase (MAPK)/extracellular signal regulated kinase (ERK), JNK/p38 signaling, Wnt and Noggle. Therefore, type I receptor functions as a downstream of type II receptor. ${ }^{193-197}$

Smad proteins, named from mothers against DPP homologs, are the major signaling mediators in the TGF- $\beta$ superfamily induced chondrogenesis. There are three types of Smad proteins: receptor-regulated Smads (R-Smads), common-partner Smads (Co-Smads) and inhibitory Smads (I-Smads). In the TGF- $\beta$ induced signaling pathway, phosphorylated type I receptor activates R-Smads, Smad2 and Smad3 specifically. Samd2/3 associate with Co-Smad (Smad4) and the complex transfers to the nucleus to regulate the expression of target genes in cooperation with transcription factors. In BMP signaling pathway, type I receptor activates Smad1/5/8 and links to Smad4. The compound of Samd1/5/8/4 moves to the nucleus and starts the target gene expression (Figure 1.10). ${ }^{193-195 ; 198-200}$

TGF- $\beta$ also induces the MAPK/ERK signaling pathway. In this pathway, binding of TGF- $\beta$ to type II receptor induces the activation of type I receptor. This results in phosphorylation of numerous kinases, such as Src (V-src sarcoma (Schmidt-Ruppin A-2) viral oncogene homolog) and Grb2 (growth factor receptor binding protein 2). The phosphorylation of Src or Grb2 activates Ras by catalyzing the exchange of GDP to GTP. Ras then binds to Raf and activates the MAPK cascade including MEK and ERK1/2 (Figure 1.11). ${ }^{201-204}$ However, the function of ERK1/2 in chondrogenesis is controversial. Yamada et al. demonstrated that activation of ERK1/2 was crucial in regulation of aggrecan gene expression induced by TGF- $\beta,{ }^{205}$ but recent studies suggested that inhibition of ERK1/2 can improve the MSC chondrogenic differentiation. ${ }^{206-208}$ One possible explanation is that activation of ERK $1 / 2$ is essential to chondrogenesis induced by TGF- $\beta$ in the early stage up to 120 minutes, but in a long culture period like 14 days, the activity of ERK1/2 is suppressed to give way to other signaling pathways. 
TGF- $\beta$ can induce JNK and p38 MAPK signaling cascades. TGF- $\beta$ activates JNK through MKK4 (MAP kinase kinase 4) and p38 MAPK through MKK3/6. Activated JNK and p38 MAPK move to the nucleus and initiate the expression of target genes with transcription factors (Figure 1.12). ${ }^{204 ; 209-213}$ More importantly, the signaling pathway of JNK and p38 is independent of Smad signaling, proven by sustained activation of JNK and p38 in a Smad3- and Smad4-deficient model. ${ }^{214 ; 215}$

TGF- $\beta$ can induce the PI3K/Akt signaling pathway. PI3K is activated by TGF- $\beta$ and then phosphorylates the downstream Akt that is independent of Smad activation ${ }^{216}$. PI3K plays an important role in cell adhesion, migration and TGF- $\beta$-induced actin filament reorganization (Figure 1.13). ${ }^{204 ; 217}$ However, the PI3K/Akt signaling was reported to antagonize Smad-mediated effects. The activated Akt can interact with Smad3 and hence prevent the translocation of Smad2/3 to the nucleus. The interaction of Akt and Smad3 can delay chondrogenic differentiation induced by TGF- $\beta .^{218-221}$

TGF- $\beta$ can activate Rho-like GTPase, including RhoA, Rac and Cdc42 to modulate cell adhesion, proliferation and survival. ${ }^{204 ; 222 ; 223}$ The activation of RhoA and Cdc42 by TGF- $\beta$ is independent of the Smads signaling pathway, indicated by the unaffected activation of p21-activated kinase (PAK) in the inhibition of Smad2/3 phosphorylation. ${ }^{224 ; 225}$ However, Smad7 is associated with the activation of Cdc42 (Figure 1.14). ${ }^{226 ; 227}$

In MSC chondrogenic differentiation, Sox9 (Sry-related high-mobility-group box 9) is the key transcriptional factor to initiate the expression of chondrogenic-specific genes and inhibits chondrocyte hypertrophy. After the activation of Smad2/3 by TGF- $\beta$, the Sox 9 and Smad2/3/4 complex translocate to the nucleus. Cooperating with other transcription factors, such as Barx2 and c-Maf, chondrogenic genes like type II collagen and aggrecan are activated to express. ${ }^{228-230}$ In vivo Sox9 plays an important role in the development of musculoskeletal tissues and organs, proven by the failure of mutant Sox9-/- mice to develop normal bone and cartilage. ${ }^{231-236}$ 


\subsection{MSC-based cartilage tissue engineering}

\subsubsection{Definition of cartilage tissue engineering}

Tissue engineering (TE) is defined as the utilization of biomaterials, cells, factors and other biochemical and biophysical stimulations to regenerate, repair or restore live tissues that have not only physiological but also biomechanical functions. ${ }^{237}$ Cells used in TE can be autologous, allogeneic and xenogeneic. Biochemical factors, such as signaling molecules and biophysical stimulation like compression or shear force, induce cells toward desired lineage tissues. Biomaterials like scaffolds function to provide support for cells and mechanical properties such as suitable stiffness and elasticity. The general procedure of TE includes the following steps. MSCs or autologous cells are harvested from patients and expanded in vitro to acquire adequate cell numbers. The expanded cells are seeded into biocompatible scaffolds and cultured in the presence of growth factor combination to induce cell proliferation and differentiation. The immature constructs containing the scaffolds and cells are cultured in a bioreactor that mimics the in vivo condition or presses suitable physical stimulations. The mature constructs (also called engineered tissue) are transplanted into patients to repair catilage defects or damaged tissues. ${ }^{237 ; 238}$ The cells used in cartilage tissue engineering include chondrocytes, ESCs and adult MSCs that were discussed previously. The growth factors in TE are the same as those used to induce chondrogenesis. Next we will focus on biocompatible scaffolds and bioreactors.

\subsubsection{Biocompatible scaffolds}

The primary function of scaffolds is to provide a temporary structure for implanted cells until new extracellular matrix is generated. The criteria for scaffolds include biocompatibility, biodegradability, mechanical stability and a porous structure that is three-dimensional and permissive for biological growth factors.

Fibrous scaffolds are the most commonly used in cartilage tissue engineering including poly glycolic acid (PGA), poly lactic acid (PLA) and the copolymer poly lactic-co-glycolic acid 
(PLGA). They have been approved by US Food and Drug Administration (FDA) for clinical use. The fibrous scaffolds have a three-dimensional structure for cell attachment, migration and proliferation. They can be degraded by hydrolysis and the degradation time is controllable. Several in vitro and in vivo studies showed that MSCs or chondrocytes seeded in scaffolds generated extracellular matrix that is identical to native hyaline cartilage. Those constructs were implanted to repair partial-thickness or full-thickness defects in articular cartilage and achieved better results than control groups. ${ }^{155 ; 239-244}$ Fibrous scaffolds are modified and improved by using nano-technology. Due to the morphological similarities of nanofibers to natural extracellular matrix, MSCs and chondrocytes can be improved to higher

proliferative rate and better generation of matrix. ${ }^{245-247}$ Composite scaffolds are developed to enhance cell attachment and chondrogenesis, such as the synthesis of PLA, chitosan and hyaluronan. ${ }^{248-250}$

Hydrogel scaffolds are also commonly used in cartilage tissue engineering that can absorb water and signaling factors, keep cell morphology and be injected into defect areas. The types of hydrogels include type I and III collagen, hyaluronan, chondroitin sulfate and poly ethylene glycol (PEG). ${ }^{251-254}$ MSCs and chondrocytes are seeded in hydrogels and successfully generate cartilage-specific matrix. The hydrogels are improved to incorporate signaling molecules such as growth factors that can spontaneously deliver TGF- $\beta 1$ to induce cell chondrogenesis and be removed by cellular enzymes. ${ }^{255 ; 256}$

\subsubsection{Bioreactors}

Hydrodynamic bioreactor is the most commonly used equipment in tissue engineering and is also called a rotating wall vessel (RWV). Cell-scaffold constructs are placed in the hydrodynamic bioreactor filled with medium and growth factor supplements, and gas exchange occurs through a permeable core. With rotation, constructs can be cultured with sufficient nutrients and subjected to a low-level shear force. ${ }^{257-259}$ Our laboratory used the hydrodynamic bioreactor to induce SDSCs into hyaline cartilage-like tissue. ${ }^{155}$ Dynamic compression bioreactor is designed to increase construct mechanical properties by enhancing cartilage-specific matrix generation. It is reported that chondrocytes seeded in 
hydrogel increased matrix synthesis though daily compressive stimulation. ${ }^{260 ; 261}$ The hydrostatic pressure bioreactor is made to mimic the contact stress in joints and increase the generation of cartilage-like matrix. ${ }^{262}$

\subsubsection{Cartilage tissue engineering using synovium-derived stem cells}

Our laboratory successfully generated tissue constructs identical to native hyaline cartilage using porcine SDSCs. ${ }^{155}$ SDSCs were collected from two young pigs and negative isolation was undertaken using CD14 specific antibody conjugated Dynabeads ${ }^{\circledR}$ or conventional expansion. SDSCs were seeded in PGA scaffold with fibrin glue and cultured in a cocktail of $10 \mathrm{ng} / \mathrm{mL}$ TGF- $\beta 1,50 \mathrm{ng} / \mathrm{mL}$ FGF-2 and $500 \mathrm{ng} / \mathrm{mL}$ IGF-I for 3 days. After that, the constructs containing SDSCs and PGA were transferred to a hydrodynamic bioreactor filled with chondrogenic medium supplemented with $10 \mathrm{ng} / \mathrm{mL}$ TGF- $\beta 1$ and $500 \mathrm{ng} / \mathrm{mL}$ IGF-I for one month.

Histology and immunohistochemistry showed that the SDSC constructs strongly expressed sulfated glycosaminoglycans and type II collagen. The hypertrophic marker, type X collagen was negative. Mechanical analysis suggested that the SDSC constructs had similar equilibrium modulus to natural hyaline cartilage. SDSC constructs with negative isolation generated 50\% more GAG and had two-fold equilibrium modulus than the constructs with conventional expansion. Immunohistochemistry confirmed that macrophage cells existed in the conventionally expanded synovial cells, but were completely removed after negative isolation (Figure 1.15).

Rabbit SDSC constructs were engineered following the same protocol above and transplanted into full-thickness femoral condyle cartilage defects in New Zealand white rabbits. After six months, cartilage morphology and histology analysis showed that the cartilage defects were filled with newly generated constructs, strongly expressing proteoglycans and type II collagen but had negative expression of type I collagen. In the control group, fibrocartilagenous tissues were found in the defects that were positive for type I collagen. Mechanical analysis showed that the defect areas repaired with SDSC constructs 
had an equilibrium modulus that was identical to the surrounding undamaged cartilage tissue and were higher than that in the control group (Figure 1.16). The engineered constructs using SDSCs successfully repaired full-thickness defects in articular cartilage. ${ }^{263}$

\subsection{Extracellular matrix}

Extracellular matrix (ECM) provides an essential microenvironment for mesenchymal stem cells and plays an important role in cell adhesion, survival, migration, proliferation, differentiation and matrix remodeling. ${ }^{174 ; 264 ; 265}$ Natural ECM provides a structural scaffold to resist tensile and compressive stress, and functions as a tight connection to the cytoskeleton of cells through cell-surface receptors to enable cells to sense and respond to mechanical and chemical signals. Stromal cells and MSCs usually reside within a specific extracellular regulatory microenvironment containing a complex mixture of ECM signals to direct cell self-renewal or differentiation. The extracellular signaling includes soluble cytokines and growth factors, components of ECM such as collagen and mineralized materials and the interaction between neighboring cells through cell surface receptors. ${ }^{266-268}$

Bone marrow, the microenvironment for osteoblasts, BMSCs and HSCs, is composed of calcified bone matrix, type I, III, IV, V and VI collagen, fibronectin, laminin, connecting proteins and large molecular weight proteoglycans. ${ }^{265 ; 269-271}$ Growth factors reside in bone marrow (such as TGF- $\beta$, IGF, BMPs) and ECM acts as a reservoir to pass signals to cells through interaction with cell surface receptors. ${ }^{272-275}$ In bone marrow, the sustainability of BMSC self-renewal and the induction of differentiation are supported by the influence of factors secreted by osteoblasts, the adhesion to ECM and the connections between MSCs and osteoblasts, HSCs and stromal cells. ${ }^{276 ; 277}$

In articular cartilage ECM not only provides structural integrity but also has physiological functions to maintain chondrocyte morphology, direct chondrocyte migration and influence immune cytokines. ${ }^{8 ; 21 ; 4 ; 159}$ The components of articular cartilage include type II collagen, proteoglycans (primarily aggrecan), hyaluronan and other matrix proteins. Cartilage ECM plays an important role in influencing the microenvironment of chondrocytes through 
cell-matrix receptors. ${ }^{4 ; 278 ; 279}$

\subsubsection{Integrin system and signaling mechanism}

Extracellular matrix has a profound influence on cell adhesion, survival, proliferation and differentiation. The interaction between cells and ECM are mainly dependent on integrins and other surface receptors. ${ }^{280-282}$ Here we focus on the integrins of mesenchymal stem cells and the integrin-dependent intracellular signaling mechanism.

Integrin receptors have a transmembrane structure and are composed of heterodimeric molecules including $\alpha$ and $\beta$ subunits. The extracellular domains have contact with ECM and the cytoplasmic domains associate with cell actin cytoskeleton and protein kinases. Currently at least $16 \alpha$ and $8 \beta$ subunits of integrins have been identified that can generate 22 distinct $\alpha \beta$ heterodimeric receptors. ${ }^{280 ; 281 ; 283 ; 284}$ Table 1.5 shows the major integrin heterodimeric receptors and their protein ligands. ${ }^{281}$

\begin{tabular}{|l|l|l|}
\hline \multicolumn{2}{|c|}{ Integrin subunits } & \\
\hline$\beta_{1}$ & $\alpha_{1}$ & Collagens, Laminins \\
\hline & $\alpha_{2}$ & Collagens, Laminins \\
\hline & $\alpha_{3}$ & Collagens, Laminins, Fibronectin, Entacin \\
\hline & $\alpha_{4}$ & Fibronectin, VCAM-1 \\
\hline & $\alpha_{5}$ & Fibronectin \\
\hline & $\alpha_{6}$ & Laminins \\
\hline & $\alpha_{7}$ & Laminins \\
\hline & $\alpha_{8}$ & Vitronectin, Fibronectin, Tenascin \\
\hline & $\alpha_{9}$ & Vitronectin, Fibronectin, Tenascin \\
\hline & $\alpha_{10}$ & Collagens \\
\hline$\beta_{2}$ & $\alpha_{11}$ & Collagens \\
\hline & $\alpha_{\mathrm{V}}$ & Vitronectin, Fibronectin \\
\hline & ICAM-1. ICAM-2, ICAM-3 \\
\hline
\end{tabular}




\begin{tabular}{|l|l|l|}
\hline & $a_{M}$ & IC3b, Fibronectin, ICAM-1 \\
\hline & $a_{X}$ & IC3b, Fibronectin \\
\hline$\beta_{3}$ & $\alpha_{D}$ & ICAM-3 \\
\hline$\beta_{4}$ & $\alpha_{6}$ & $\begin{array}{l}\text { Fibronectin, Fibrinogen, von Willebrand factor, } \\
\text { Vitroectin, Thrombospondin, Tenasin }\end{array}$ \\
\hline$\beta_{5}$ & $\alpha_{V}$ & $\begin{array}{l}\text { Fibronectin, Fibrinogen, von Willebrand factor, } \\
\text { Vitroectin, Thrombospondin, Osteopontin, Collagens }\end{array}$ \\
\hline$\beta_{6}$ & $a_{V}$ & Laminins \\
\hline$\beta_{7}$ & $\alpha_{4}$ & Vitronectin, Fibronectin \\
\hline & $a_{E}$ & Tenasin, Fibronectin \\
\hline$\beta_{8}$ & $a_{V}$ & Fibronectin, VCAM-1 \\
\hline & & E-cadherin \\
\hline
\end{tabular}

Table 1.5 Integrin receptors and the protein ligands ${ }^{281}$

Due to the short structure and lack of enzymatic function of the cytoplasmic domains of integrins, extracellular signaling transduction must be accompanied by cytoskeletal actins, cytoplasmic kinases and transmembrane growth factor receptors. After contacting with ECM, integrins cluster in cell membrane, become a signaling complex and associate with cytoskeletal actin filaments. The reorganized actin filaments in turn accelerate the clustering of integrin with a positive feedback mechanism. Finally, ECM, integrins and the cytoskeletal actins aggregate into a large complex through cell membrane to transduce extracellular signals into cells. ${ }^{281 ; 282 ; 285}$

Integrins activate several tyrosine kinase; one of those, focal adhesion kinase (FAK), has been studied in depth. After integrin clustering, the $\beta$ subunit activated FAK directly or through cytoskeletal proteins such as talin and paxillin. FAK autophosphorylates $\mathrm{Tyr}^{397}$ and creates a binding site for Src homology 2 (SH2) domain of Src or Fyn. Src then phosphorylates FAK at $\mathrm{Tyr}^{925}$ and activates Grb2 and Ras. Phosphorylated FAK eventually activates MAPK 
cascades and modifies cytoskeletal actin filaments. ${ }^{281 ; 282 ; 286}$ Activation of MAPK cascades can also be FAK-independent, in which the activation is initiated by integrin $\alpha_{5} \beta_{1}, \alpha_{v} \beta_{3}, \alpha_{2} \beta_{1}$, $\alpha_{v} \beta_{6}$ and $\alpha_{6} \beta_{4}$ respectively, when cells adhere to ECM proteins such as fibronectin, collagens, laminins, virtonectin and tenascin. It is believed that FAK-related proteins such as CAK $\beta$ and PYK2/RAFTK may compensate for FAK to activate the MAPK signaling pathway. ${ }^{280 ; 282 ; 287 ; 288}$

\subsubsection{Typical ECM proteins and their integrin receptors}

Fibronectin is one of the major ECM proteins in bone marrow and articular cartilage, and it is most commonly used as a substrate in MSC expansion in vitro. ${ }^{289-291}$ Two different integrins, $\alpha_{5} \beta_{1}$ and $\alpha_{v} \beta_{3}$, are identified as the receptors to fibronectin, which recognize a short motif composed by three amino acid residues (RGD: arginine, glysine and aspartic acid) in

fibronectin. ${ }^{292-295}$ In addition, integrins of $\alpha_{\| 1 b} \beta_{3}, \alpha_{v} \beta_{5}, \alpha_{v} \beta_{8}, \alpha_{v} \beta_{6}$ and $\alpha_{v} \beta_{1}$ are shown to contact fibronectin by binding to the RGD motif. $\alpha_{4} \beta_{1}$ and $\alpha_{4} \beta_{7}$ integrins recognize fibronectin through an RGD-independent motif but LDV or REDV motif. ${ }^{296-298}$ The binding of integrins to fibronectin induces FAK phosphorylation and activation of the MAPK cascade. ${ }^{292 ; 293}$ Interestingly many proteoglycans and glycosaminoglycans bind to fibronectin and the interaction between integrins and proteoglycans are associated with syndecans. Syndecans 1, 2 and 3 are only found in specific tissues whereas syndencan 4 is ubiquitous. Integrin heterodimers bind to proteoglycans with the cooperation of syndecans and syndecans activate intracellular signaling transduction and induce focal adhesion. ${ }^{292 ; 299-303}$

Collagen is a main component in bone and cartilage that is recognized by several receptors. Four collagen receptors of integrin heterodimers are identified including $\alpha_{1} \beta_{1}, \alpha_{2} \beta_{1}, \alpha_{10} \beta_{1}$ and $\alpha_{11} \beta_{1} \cdot{ }^{293 ; 299 ; 300 ; 304}$ Integrin $\alpha_{1} \beta_{1}$, expressed in MSCs, is considered the receptor to type IV collagen and has a high affinity to non-fibrillar collagens. ${ }^{305-307}$ Integrin $\alpha_{2} \beta_{1}$ is widely expressed and binds to collagen fibrils and fibrillar collagens. ${ }^{308-310}$ Integrin $\alpha_{10} \beta_{1}$ is found in cartilage chondrocytes ${ }^{311}$ and integrin $\alpha_{11} \beta_{1}$ plays an important role in tooth development. ${ }^{312}$ Integrin collagen receptors can recognize proteoglycans with an association with syndecans that is similar to fibronectin receptors. ${ }^{313 ; 314}$ In addition to integrin collagen receptors, discoidin domain receptors (DDRs) and glycoprotein (GP) VI can also bind to collagens and activate 
intracellular signaling pathways. ${ }^{315-317}$

Laminins are important components of the basement membrane together with type IV collagen. Four integrin heterodimers are the receptors including $\alpha_{3} \beta_{1}, \alpha_{6} \beta_{1}, \alpha_{6} \beta_{4}$ and $\alpha_{7} \beta_{1}{ }^{293}$ Integrin $\alpha_{3} \beta_{1}$ is essential in the development of kidneys, lungs and skin. ${ }^{318 ; 319}$ Integrin $\alpha_{7} \beta_{1}$ is found in muscle tissue and is important to muscle development ${ }^{320}$. Integrin $\alpha_{6} \beta_{4}$ is found in epithelial cells and binds to the basement membranes. ${ }^{321 ; 322}$ Besides integrin receptors, a-dystroglycan functions as a laminin receptor that is also known as 67kD laminin receptor due to its molecular weight. ${ }^{292 ; 323 ; 324}$

\subsubsection{D vs. $2 D$ ECM and distinct cellular response}

The current understanding of interaction between cells and ECM is based on studies of focal and fibrillar adhesions. Research on cell adhesion, migration, proliferation and differentiation is primarily derived from in vitro two-dimensional (2D) tissue culture substrate. However, in vivo three-dimensional (3D) ECM provides a different support to cells and induces distinct intracellular signaling transductions to influence cell adhesion, motility and proliferation. The studies of 3D ECM and the induced cell response can improve MSC-based tissue regeneration including MSC in vitro expansion, tissue-specific differentiation and the design of novel scaffolds. ${ }^{264 ; 325 ; 326}$

A novel cell-deposited 3D ECM provides a new approach to study the interaction between cells and ECM. ${ }^{94 ; 95 ; 325}$ Traditional 2D ECM involves the deposition of soluble matrix proteins such as fibronectin, collagen and laminins on culture surface. Compared to traditional 2D ECM, this natural 3D ECM composes multiple matrix proteins such as type I and III collagen, fibronectin, heparin sulfate proteoglycans, elastin, laminins and decorin etc. In the structure, 3D ECM is more pliable than 2D ECM, has higher fibrillar matrix and contains the space originally occupied by the feeder cells generating matrix. ${ }^{325 ; 327 ; 328}$

An assay using human fibroblasts grown on 3D and 2D ECM showed that the cells attached to 3D ECM six-fold faster compared to the substrates of fibronectin, collagen, laminin or 3D 
collagen gel. ${ }^{264}$ In 2D culture, cells adhere to ECM primarily on focal adhesion involving integrin $\alpha_{v} \beta_{3}$ and fibrillar adhesion depending on integrin $\alpha_{5} \beta_{1}$. On 3D ECM, cells adhesion involves integrin $\alpha_{5} \beta_{1}$ and $\alpha_{2} \beta_{1}$ and the absence of integrin $\alpha_{v} \beta_{3}$. The focal adhesion is completely associated with fibronectin-containing fibrils that can be independent of fibronectin in 2D culture. ${ }^{264 ; 325 ; 326}$ Meanwhile, phosphorylation of FAK decreases when cells are cultured on 3D ECM but the activity of SrC and ERK1/2 increases, indicating that cell adhesion and protein kinase activity are different between the 3D and 2D environments. ${ }^{329}$

The cell proliferation rate on 3D ECM increases at least two-fold in human fibroblasts ${ }^{325}$ and 12-fold in porcine SDSCs ${ }^{95}$ compared to cells in 2D culture. The cell migration on 3D ECM is directional rather than the random migration on 2D culture. Rac is considered to control cell migration and Rac activity was proven to decrease on 3D ECM. The lower activity of Rac induced more directional migration. ${ }^{264 ; 325 ; 326}$ Another reason for the directional movement is that $3 \mathrm{D}$ ECM is more pliable and contains more space than $2 \mathrm{D} \mathrm{ECM}$; cells can sense the rigidity of ECM and move to areas with higher rigidity. ${ }^{264 ; 325 ; 330 ; 331}$ However, the work on 3D ECM and cell response is just beginning. Using 3D ECM is a new approach to study cell behavior, cancer mechanisms, drug delivery and to improve MSC-based tissue regeneration.

\subsection{Challenges in cartilage tissue regeneration}

Mesenchymal stem cells provide a promising cell source for cartilage tissue regeneration due to their self-renewal ability and multipotential differentiation capacity. However, there are several challenges facing the current application of MSC-based cartilage regeneration.

The lack of a unique MSC surface marker makes it difficult to identify and isolate MSCs from cell pools. Even though it is reported that MSCs must show positive for CD90, CD105 and CD73 but negative for CD45, CD11b, CD14 or HLA-DR, the percentage of positive surface markers changes in different MSCs from different tissue sources. ${ }^{332}$ SSEA-4 is demonstrated as a positive surface marker in MSCs and the sorting of SSEA- $4^{+}$cells indeed enhanced differentiation including osteogenesis, adipogenesis and chondrogenesis. ${ }^{333}$ The SSEA-4 positive isolation method is mainly limited to BMSCs, so future work will concentrate on 
testing different tissue-source MSCs. Our negative isolation method using Dynabeads conjugated with CD14 antibody is an indirect way to purify SDSCs. ${ }^{148 ; 155}$ This negative isolation has some drawbacks including the removal of excessive cells up to $50 \%$ of the total population and unbound cells that are a mixed population of SDSCs and synovial fibroblasts. In future, it is important to identify unique cell surface markers for MSCs and develop a new isolation method to purify MSCs from other fibroblasts and immune cells.

Self-renewal, the process by which MSCs proliferate and create more cells, is one of the important properties of MSCs. Maintaining self-renewal ability in vitro is crucial to MSCs as that it provides sufficient cells for tissue regeneration in vivo. Conventional MSC in vitro culture requires weekly passaging to retain proliferation rate and preserve future differentiation potential. However, the late passages of MSCs not only fail to proliferate ${ }^{140 ; 152 ; 154}$ but also lose multipotency except for differentiation into adipocytes. ${ }^{334}$

Another important property of MSCs is the stemness that is defined by the potentials of MSCS to differentiate into multiple tissues. The loss of multipotency in late-passage MSCs is possibly due to exposure to enzymes such as trypsin that damages cell surface proteins. ${ }^{335-338}$ Another explanation is spontaneous differentiation in the expansion period affected by serum and the 2D culture environment. Spontaneous differentiation is different from induced differentiation which is controlled by growth factors. Cells express multiple differentiation growth factors and cytokines in spontaneous differentiation that significantly reduce the sensitivity and effectiveness of cells in the future induced differentiation. ${ }^{94 ; 271 ; 339}$ Tuan and colleagues tried to identify the signature genes that maintain MSC self-renewal and multipotency. Five specific genes were found expressed in undifferentiated MSCs but absent in differentiated cells, including actin filament associated protein, frizzled 7 , dickkopf 3 , protein tyrosine phosphatase receptor $\mathrm{F}$ and RAB3B. The interference of these genes by siRNA could affect MSC proliferative rate and multipotency. ${ }^{340}$ However, our understanding of the stemness of MSCs is poor and it is important to improve the in vitro culture of MSCs to improve self-renewal and multipotency. 


\section{REFERENCES}

1. Little K. Nature of osteopetrosis. Br.Med.J. 1969;2:49-50.

2. Mow VC, Ateshian GA, Spilker RL. Biomechanics of diarthrodial joints: a review of twenty years of progress. J.Biomech.Eng 1993;115:460-7.

3. Curl WW, Krome J, Gordon ES et al. Cartilage injuries: a review of 31,516 knee arthroscopies. Arthroscopy 1997;13:456-60.

4. Muir H. The chondrocyte, architect of cartilage. Biomechanics, structure, function and molecular biology of cartilage matrix macromolecules. Bioessays $1995 ; 17: 1039-48$

5. Nelson L, Fairclough J, Archer CW. Use of stem cells in the biological repair of articular cartilage. Expert. Opin.Biol.Ther. 2010;10:43-55.

6. Swieszkowski W, Tuan $\mathrm{BH}$, Kurzydlowski $\mathrm{KJ}$ et al. Repair and regeneration of osteochondral defects in the articular joints. Biomol.Eng 2007;24:489-95.

7. Barbero A, Grogan S, Schafer D et al. Age related changes in human articular chondrocyte yield, proliferation and post-expansion chondrogenic capacity. Osteoarthritis. Cartilage. 2004;12:476-84.

8. Fritz J, Janssen P, Gaissmaier C et al. Articular cartilage defects in the knee--basics, therapies and results. Injury 2008;39 Suppl 1:S50-S57.

9. Matthew J.Ravosa, Elisabeth K.Lopez, Rachel A.Menegaz et al. Using "Mighty Mouse" to understand masticatory plasticity: myostatin-deficient mice and musculoskeletal function. Integrative and Comparative Biology 2008;48:345-59.

10. Ding $M$, Dalstra $M$, Linde $F$ et al. Mechanical properties of the normal human tibial cartilage-bone complex in relation to age. Clin.Biomech.(Bristol., Avon.) 1998;13:351-8.

11. Rains JK, Bert JL, Roberts $\mathrm{CR}$ et al. Mechanical properties of human tracheal cartilage. J.Appl.Physiol 1992;72:219-25.

12. Ding $M$, Dalstra $M$, Linde $F$ et al. Changes in the stiffness of the human tibial cartilage-bone complex in early-stage osteoarthrosis. Acta Orthop.Scand. 1998;69:358-62. 
13. Obeid EM, Adams MA, Newman JH. Mechanical properties of articular cartilage in knees with unicompartmental osteoarthritis. J.Bone Joint Surg.Br. 1994;76:315-9.

14. Khan IM, Gilbert SJ, Singhrao SK et al. Cartilage integration: evaluation of the reasons for failure of integration during cartilage repair. A review. Eur.Cell Mater. 2008;16:26-39.

15. Hjelle K, Solheim E, Strand T et al. Articular cartilage defects in 1,000 knee arthroscopies. Arthroscopy 2002;18:730-4.

16. Widuchowski W, Kusz D, Widuchowski J et al. [Analysis of articular cartilage lesions in 5114 knee arthroscopies]. Chir Narzadow.Ruchu.Ortop.Pol. 2006;71:117-21.

17. Widuchowski W, Widuchowski J, Trzaska T. Articular cartilage defects: study of 25,124 knee arthroscopies. Knee. 2007;14:177-82.

18. Jackson DW, Lalor PA, Aberman HM et al. Spontaneous repair of full-thickness defects of articular cartilage in a goat model. A preliminary study. J.Bone Joint Surg.Am. 2001;83-A:53-64.

19. Beris $A E$, Lykissas MG, Papageorgiou $C D$ et al. Advances in articular cartilage repair. Injury 2005;36 Suppl 4:S14-S23.

20. OUTERBRIDGE RE. The etiology of chondromalacia patellae. J.Bone Joint Surg.Br. 1961;43-B:752-7.

21. Martel-Pelletier J, Boileau C, Pelletier JP et al. Cartilage in normal and osteoarthritis conditions. Best.Pract.Res.Clin.Rheumatol. 2008;22:351-84.

22. Mollenhauer JA. Perspectives on articular cartilage biology and osteoarthritis. Injury 2008;39 Suppl 1:S5-12.

23. Goldring MB, Goldring SR. Articular cartilage and subchondral bone in the pathogenesis of osteoarthritis. Ann. N.Y.Acad.Sci. 2010;1192:230-7.

24. Horton WE, Jr., Bennion P, Yang L. Cellular, molecular, and matrix changes in cartilage during aging and osteoarthritis. J.Musculoskelet.Neuronal.Interact. 2006;6:379-81.

25. Loeser RF. Molecular mechanisms of cartilage destruction in osteoarthritis. J.Musculoskelet.Neuronal.Interact. 2008;8:303-6.

26. Kotlarz H, Gunnarsson $\mathrm{CL}$, Fang $\mathrm{H}$ et al. Osteoarthritis and absenteeism costs: 
evidence from US National Survey Data. J.Occup.Environ.Med. 2010;52:263-8.

27. Kotlarz H, Gunnarsson CL, Fang $\mathrm{H}$ et al. Insurer and out-of-pocket costs of osteoarthritis in the US: evidence from national survey data. Arthritis Rheum. 2009;60:3546-53.

28. Anderson PA, Rouleau JP. Intervertebral disc arthroplasty. Spine (Phila Pa 1976.) 2004;29:2779-86

29. Hsieh AH, Twomey JD. Cellular mechanobiology of the intervertebral disc: new directions and approaches. J.Biomech. 2010;43:137-45.

30. Urban JP, Roberts S. Degeneration of the intervertebral disc. Arthritis Res.Ther. 2003;5:120-30.

31. Setton LA, Chen J. Cell mechanics and mechanobiology in the intervertebral disc. Spine (Phila Pa 1976.) 2004;29:2710-23.

32. Roughley PJ. Biology of intervertebral disc aging and degeneration: involvement of the extracellular matrix. Spine (Phila Pa 1976.) 2004;29:2691-9.

33. Smith LJ, Fazzalari NL. The elastic fibre network of the human lumbar anulus fibrosus: architecture, mechanical function and potential role in the progression of intervertebral disc degeneration. Eur.Spine J. 2009;18:439-48.

34. Masuda K, Oegema TR, Jr., An HS. Growth factors and treatment of intervertebral disc degeneration. Spine (Phila Pa 1976.) 2004;29:2757-69.

35. Le Maitre $\mathrm{CL}$, Freemont $\mathrm{AJ}$, Hoyland JA. The role of interleukin-1 in the pathogenesis of human intervertebral disc degeneration. Arthritis Res.Ther. 2005; 7:R732-R745.

36. Hastreiter D, Ozuna RM, Spector M. Regional variations in certain cellular characteristics in human lumbar intervertebral discs, including the presence of alpha-smooth muscle actin. J.Orthop.Res. 2001;19:597-604.

37. Maroudas A, Stockwell RA, Nachemson A et al. Factors involved in the nutrition of the human lumbar intervertebral disc: cellularity and diffusion of glucose in vitro. J.Anat. $1975 ; 120: 113-30$

38. Boos N, Weissbach $\mathrm{S}$, Rohrbach $\mathrm{H}$ et al. Classification of age-related changes in lumbar intervertebral discs: 2002 Volvo Award in basic science. Spine (Phila Pa 
1976.) 2002;27:2631-44.

39. Gruber HE, Ingram JA, Norton $\mathrm{HJ}$ et al. Senescence in cells of the aging and degenerating intervertebral disc: immunolocalization of senescence-associated beta-galactosidase in human and sand rat discs. Spine (Phila Pa 1976.) 2007;32:321-7.

40. Roberts S, Evans EH, Kletsas D et al. Senescence in human intervertebral discs. Eur.Spine J. 2006;15 Suppl 3:S312-S316.

41. Zhao CQ, Wang LM, Jiang LS et al. The cell biology of intervertebral disc aging and degeneration. Ageing Res.Rev. 2007;6:247-61.

42. Gaissmaier C, Koh JL, Weise K et al. Future perspectives of articular cartilage repair. Injury 2008;39 Suppl 1:S114-S120.

43. Marlovits $\mathrm{S}$, Zeller $\mathrm{P}$, Singer $\mathrm{P}$ et al. Cartilage repair: generations of autologous chondrocyte transplantation. Eur.J.Radiol. 2006;57:24-31.

44. Steadman JR, Rodkey WG, Rodrigo JJ. Microfracture: surgical technique and rehabilitation to treat chondral defects. Clin.Orthop.Relat Res. 2001;S362-S369.

45. Hunziker EB. Articular cartilage repair: basic science and clinical progress. A review of the current status and prospects. Osteoarthritis. Cartilage. 2002;10:432-63.

46. Hangody L, Fules P. Autologous osteochondral mosaicplasty for the treatment of full-thickness defects of weight-bearing joints: ten years of experimental and clinical experience. J.Bone Joint Surg.Am. 2003;85-A Suppl 2:25-32.

47. Matsusue Y, Yamamuro T, Hama H. Arthroscopic multiple osteochondral transplantation to the chondral defect in the knee associated with anterior cruciate ligament disruption. Arthroscopy 1993;9:318-21.

48. Amiel $D$, Coutts $R D$, Abel $M$ et al. Rib perichondrial grafts for the repair of full-thickness articular-cartilage defects. A morphological and biochemical study in rabbits. J.Bone Joint Surg.Am. 1985;67:911-20.

49. Huntley JS, Bush PG, McBirnie JM et al. Chondrocyte death associated with human femoral osteochondral harvest as performed for mosaicplasty. J.Bone Joint Surg.Am. 2005;87:351-60.

50. Brittberg M, Lindahl A, Nilsson A et al. Treatment of deep cartilage defects in the 
knee with autologous chondrocyte transplantation. N.Engl.J.Med. 1994;331:889-95.

51. Dell'Accio F, Vanlauwe J, Bellemans $\mathrm{J}$ et al. Expanded phenotypically stable chondrocytes persist in the repair tissue and contribute to cartilage matrix formation and structural integration in a goat model of autologous chondrocyte implantation. J.Orthop.Res. 2003;21:123-31.

52. Brittberg $M$, Peterson $L$, Sjogren-Jansson $E$ et al. Articular cartilage engineering with autologous chondrocyte transplantation. A review of recent developments. J.Bone Joint Surg.Am. 2003;85-A Suppl 3:109-15.

53. Benya PD, Shaffer JD. Dedifferentiated chondrocytes reexpress the differentiated collagen phenotype when cultured in agarose gels. Cell 1982;30:215-24.

54. Loty S, Sautier JM, Loty C et al. Cartilage formation by fetal rat chondrocytes cultured in alginate beads: a proposed model for investigating tissue-biomaterial interactions. J.Biomed.Mater.Res. 1998;42:213-22.

55. van der Kraan PM, Buma P, van KT et al. Interaction of chondrocytes, extracellular matrix and growth factors: relevance for articular cartilage tissue engineering. Osteoarthritis. Cartilage. 2002;10:631-7.

56. von der MK, Gauss V, von der MH et al. Relationship between cell shape and type of collagen synthesised as chondrocytes lose their cartilage phenotype in culture. Nature 1977;267:531-2.

57. Sakai D. Future perspectives of cell-based therapy for intervertebral disc disease. Eur.Spine J. 2008;17 Suppl 4:452-8.

58. Nomura T, Mochida J, Okuma $M$ et al. Nucleus pulposus allograft retards intervertebral disc degeneration. Clin.Orthop.Relat Res. 2001;94-101.

59. Okuma M, Mochida J, Nishimura K et al. Reinsertion of stimulated nucleus pulposus cells retards intervertebral disc degeneration: an in vitro and in vivo experimental study. J. Orthop.Res. 2000;18:988-97.

60. Yamamoto Y, Mochida J, Sakai D et al. Upregulation of the viability of nucleus pulposus cells by bone marrow-derived stromal cells: significance of direct cell-to-cell contact in coculture system. Spine (Phila Pa 1976.) 2004;29:1508-14.

61. Gorensek M, Jaksimovic C, Kregar-Velikonja N et al. Nucleus pulposus repair with 
cultured autologous elastic cartilage derived chondrocytes. Cell Mol.Biol.Lett. 2004;9:363-73.

62. Mwale F, Roughley P, Antoniou J. Distinction between the extracellular matrix of the nucleus pulposus and hyaline cartilage: a requisite for tissue engineering of intervertebral disc. Eur.Cell Mater. 2004;8:58-63.

63. Evans MJ, Kaufman MH. Establishment in culture of pluripotential cells from mouse embryos. Nature 1981;292:154-6.

64. Martin GR. Isolation of a pluripotent cell line from early mouse embryos cultured in medium conditioned by teratocarcinoma stem cells. Proc.NatI.Acad.Sci.U.S.A 1981;78:7634-8.

65. Thomson JA, Itskovitz-Eldor J, Shapiro SS et al. Embryonic stem cell lines derived from human blastocysts. Science 1998;282:1145-7.

66. Brignier AC, Gewirtz AM. Embryonic and adult stem cell therapy. J.Allergy Clin.Immunol. 2010;125:S336-S344.

67. Klimanskaya I, Chung Y, Becker S et al. Human embryonic stem cell lines derived from single blastomeres. Nature 2006;444:481-5.

68. Deb KD, Sarda K. Human embryonic stem cells: preclinical perspectives. J.Transl.Med. 2008;6:7.

69. Chung $\mathrm{Y}$, Klimanskaya I, Becker S et al. Embryonic and extraembryonic stem cell lines derived from single mouse blastomeres. Nature 2006;439:216-9.

70. Levenberg S, Huang NF, Lavik E et al. Differentiation of human embryonic stem cells on three-dimensional polymer scaffolds. Proc.Natl.Acad.Sci.U.S.A 2003;100:12741-6.

71. Jukes JM, Both SK, Leusink $A$ et al. Endochondral bone tissue engineering using embryonic stem cells. Proc.Natl.Acad.Sci.U.S.A 2008;105:6840-5.

72. Lerou $\mathrm{PH}$, Yabuuchi $\mathrm{A}$, Huo $\mathrm{H}$ et al. Human embryonic stem cell derivation from poor-quality embryos. Nat.Biotechnol. 2008;26:212-4.

73. Pozzobon M, Ghionzoli M, De CP. ES, iPS, MSC, and AFS cells. Stem cells exploitation for Pediatric Surgery: current research and perspective. Pediatr.Surg.Int. 2010;26:3-10. 
74. Puymirat E, Geha R, Tomescot A et al. Can mesenchymal stem cells induce tolerance to cotransplanted human embryonic stem cells? Mol.Ther. 2009;17:176-82.

75. Vogel G, Holden C. Stem cells. Ethics questions add to concerns about NIH lines. Science 2008;321:756-7.

76. CONLON HJ. Embryonic mesenchymal tumor of heart; report of a case. Am.J.Clin.Pathol. 1956;26:297-300.

77. Takahashi $\mathrm{K}$, Tanabe $\mathrm{K}$, Ohnuki $\mathrm{M}$ et al. Induction of pluripotent stem cells from adult human fibroblasts by defined factors. Cell 2007;131:861-72.

78. Yu J, Vodyanik MA, Smuga-Otto $\mathrm{K}$ et al. Induced pluripotent stem cell lines derived from human somatic cells. Science 2007;318:1917-20.

79. Meissner A, Wernig M, Jaenisch R. Direct reprogramming of genetically unmodified fibroblasts into pluripotent stem cells. Nat.Biotechnol. 2007;25:1177-81.

80. Stadtfeld $\mathrm{M}$, Nagaya $\mathrm{M}$, Utikal $\mathrm{J}$ et al. Induced pluripotent stem cells generated without viral integration. Science 2008;322:945-9.

81. Okita $\mathrm{K}$, Nakagawa $\mathrm{M}$, Hyenjong $\mathrm{H}$ et al. Generation of mouse induced pluripotent stem cells without viral vectors. Science 2008;322:949-53.

82. Zhou H, Wu S, Joo JY et al. Generation of induced pluripotent stem cells using recombinant proteins. Cell Stem Cell 2009;4:381-4.

83. Kim D, Kim CH, Moon Jl et al. Generation of human induced pluripotent stem cells by direct delivery of reprogramming proteins. Cell Stem Cell 2009;4:472-6.

84. Eminli S, Foudi A, Stadtfeld M et al. Differentiation stage determines potential of hematopoietic cells for reprogramming into induced pluripotent stem cells. Nat.Genet. 2009;41:968-76.

85. Ogawa M, Larue AC, Watson $P M$ et al. Hematopoietic stem cell origin of mesenchymal cells: opportunity for novel therapeutic approaches. Int.J.Hematol. 2010;91:353-9.

86. Jing $\mathrm{D}$, Fonseca $\mathrm{AV}$, Alakel $\mathrm{N}$ et al. Hematopoietic stem cells in co-culture with mesenchymal stromal cells--modeling the niche compartments in vitro. Haematologica 2010;95:542-50. 
87. Drouet M, Mourcin F, Grenier N et al. Mesenchymal stem cells rescue CD34+ cells from radiation-induced apoptosis and sustain hematopoietic reconstitution after coculture and cografting in lethally irradiated baboons: is autologous stem cell therapy in nuclear accident settings hype or reality? Bone Marrow Transplant. 2005;35:1201-9.

88. Fukuda K, Fujita J. Mesenchymal, but not hematopoietic, stem cells can be mobilized and differentiate into cardiomyocytes after myocardial infarction in mice. Kidney Int. 2005;68:1940-3.

89. Shiozawa $\mathrm{Y}$, Havens $\mathrm{AM}$, Pienta $\mathrm{KJ}$ et al. The bone marrow niche: habitat to hematopoietic and mesenchymal stem cells, and unwitting host to molecular parasites. Leukemia 2008;22:941-50.

90. Pereira RF, Halford KW, O'Hara MD et al. Cultured adherent cells from marrow can serve as long-lasting precursor cells for bone, cartilage, and lung in irradiated mice. Proc.Natl.Acad.Sci.U.S.A 1995;92:4857-61.

91. Pittenger MF, Mackay AM, Beck SC et al. Multilineage potential of adult human mesenchymal stem cells. Science 1999;284:143-7.

92. De BC, Dell'Accio F, Tylzanowski $P$ et al. Multipotent mesenchymal stem cells from adult human synovial membrane. Arthritis Rheum. 2001;44:1928-42.

93. Gimble JM. Adipose tissue-derived therapeutics. Expert.Opin.Biol.Ther. 2003;3:705-13.

94. Chen XD, Dusevich V, Feng JQ et al. Extracellular matrix made by bone marrow cells facilitates expansion of marrow-derived mesenchymal progenitor cells and prevents their differentiation into osteoblasts. J.Bone Miner.Res. 2007;22:1943-56.

95. He F, Chen X, Pei M. Reconstruction of an in vitro tissue-specific microenvironment to rejuvenate synovium-derived stem cells for cartilage tissue engineering. Tissue Eng Part A 2009;15:3809-21.

96. Min JY, Sullivan MF, Yang $Y$ et al. Significant improvement of heart function by cotransplantation of human mesenchymal stem cells and fetal cardiomyocytes in postinfarcted pigs. Ann.Thorac.Surg. 2002;74:1568-75.

97. Richardson SM, Hoyland JA, Mobasheri R et al. Mesenchymal stem cells in 
regenerative medicine: opportunities and challenges for articular cartilage and intervertebral disc tissue engineering. J.Cell Physiol 2010;222:23-32.

98. Beyth S, Borovsky Z, Mevorach D et al. Human mesenchymal stem cells alter antigen-presenting cell maturation and induce T-cell unresponsiveness. Blood 2005;105:2214-9.

99. Devine SM, Bartholomew AM, Mahmud $\mathrm{N}$ et al. Mesenchymal stem cells are capable of homing to the bone marrow of non-human primates following systemic infusion. Exp. Hematol. 2001;29:244-55.

100. Maitra B, Szekely E, Gjini K et al. Human mesenchymal stem cells support unrelated donor hematopoietic stem cells and suppress T-cell activation. Bone Marrow Transplant. 2004;33:597-604.

101. Tsutsumi S, Shimazu A, Miyazaki $\mathrm{K}$ et al. Retention of multilineage differentiation potential of mesenchymal cells during proliferation in response to FGF. Biochem.Biophys.Res. Commun. 2001;288:413-9.

102. Krampera M, Pizzolo G, Aprili G et al. Mesenchymal stem cells for bone, cartilage, tendon and skeletal muscle repair. Bone 2006;39:678-83.

103. Salem HK, Thiemermann C. Mesenchymal stromal cells: current understanding and clinical status. Stem Cells 2010;28:585-96.

104. Muraglia A, Cancedda R, Quarto R. Clonal mesenchymal progenitors from human bone marrow differentiate in vitro according to a hierarchical model. J.Cell Sci. 2000;113 ( Pt 7):1161-6.

105. Owen M, Friedenstein AJ. Stromal stem cells: marrow-derived osteogenic precursors. Ciba Found.Symp. 1988;136:42-60.

106. Piersma AH, Brockbank KG, Ploemacher RE et al. Characterization of fibroblastic stromal cells from murine bone marrow. Exp. Hematol. 1985;13:237-43.

107. Prockop DJ. Marrow stromal cells as stem cells for nonhematopoietic tissues. Science 1997;276:71-4.

108. Jaiswal N, Haynesworth SE, Caplan Al et al. Osteogenic differentiation of purified, culture-expanded human mesenchymal stem cells in vitro. J.Cell Biochem. 1997;64:295-312. 
109. Johnstone B, Hering TM, Caplan Al et al. In vitro chondrogenesis of bone marrow-derived mesenchymal progenitor cells. Exp. Cell Res. 1998;238:265-72.

110. Kelly KA, Gimble JM. 1,25-Dihydroxy vitamin D3 inhibits adipocyte differentiation and gene expression in murine bone marrow stromal cell clones and primary cultures. Endocrinology 1998;139:2622-8.

111. Wakitani S, Saito T, Caplan Al. Myogenic cells derived from rat bone marrow mesenchymal stem cells exposed to 5-azacytidine. Muscle Nerve 1995;18:1417-26.

112. Deng W, Obrocka M, Fischer I et al. In vitro differentiation of human marrow stromal cells into early progenitors of neural cells by conditions that increase intracellular cyclic AMP. Biochem.Biophys.Res. Commun. 2001;282:148-52.

113. Wu Y, Zhao RC, Tredget EE. Concise review: bone marrow-derived stem/progenitor cells in cutaneous repair and regeneration. Stem Cells 2010;28:905-15.

114. Colter DC, Sekiya I, Prockop DJ. Identification of a subpopulation of rapidly self-renewing and multipotential adult stem cells in colonies of human marrow stromal cells. Proc.Natl.Acad.Sci.U.S.A 2001;98:7841-5.

115. Prockop DJ, Sekiya I, Colter DC. Isolation and characterization of rapidly self-renewing stem cells from cultures of human marrow stromal cells. Cytotherapy. 2001;3:393-6.

116. Sekiya I, Larson BL, Smith JR et al. Expansion of human adult stem cells from bone marrow stroma: conditions that maximize the yields of early progenitors and evaluate their quality. Stem Cells 2002;20:530-41.

117. Buhring HJ, Battula VL, Treml S et al. Novel markers for the prospective isolation of human MSC. Ann.N.Y.Acad.Sci. 2007;1106:262-71.

118. da Silva ML, Chagastelles PC, Nardi NB. Mesenchymal stem cells reside in virtually all post-natal organs and tissues. J.Cell Sci. 2006;119:2204-13.

119. Centeno CJ, Kisiday J, Freeman M et al. Partial regeneration of the human hip via autologous bone marrow nucleated cell transfer: A case study. Pain Physician 2006;9:253-6.

120. Khanal GP, Garg M, Singh GK. A prospective randomized trial of percutaneous marrow injection in a series of closed fresh tibial fractures. Int.Orthop. 
2004;28:167-70.

121. Wakitani S, Goto T, Pineda SJ et al. Mesenchymal cell-based repair of large, full-thickness defects of articular cartilage. J.Bone Joint Surg.Am. 1994;76:579-92.

122. Murphy JM, Fink DJ, Hunziker EB et al. Stem cell therapy in a caprine model of osteoarthritis. Arthritis Rheum. 2003;48:3464-74.

123. Wakitani S, Imoto K, Yamamoto T et al. Human autologous culture expanded bone marrow mesenchymal cell transplantation for repair of cartilage defects in osteoarthritic knees. Osteoarthritis. Cartilage. 2002;10:199-206.

124. Toma C, Pittenger MF, Cahill KS et al. Human mesenchymal stem cells differentiate to a cardiomyocyte phenotype in the adult murine heart. Circulation 2002;105:93-8.

125. Wang JS, Shum-Tim D, Galipeau J et al. Marrow stromal cells for cellular cardiomyoplasty: feasibility and potential clinical advantages. J.Thorac. Cardiovasc.Surg. 2000;120:999-1005.

126. Hauner H, Entenmann G, Wabitsch $\mathrm{M}$ et al. Promoting effect of glucocorticoids on the differentiation of human adipocyte precursor cells cultured in a chemically defined medium. J.Clin. Invest 1989;84:1663-70.

127. Deslex S, Negrel R, Vannier C et al. Differentiation of human adipocyte precursors in a chemically defined serum-free medium. Int.J.Obes. 1987;11:19-27.

128. Wickham MQ, Erickson GR, Gimble JM et al. Multipotent stromal cells derived from the infrapatellar fat pad of the knee. Clin.Orthop. Relat Res. 2003;196-212.

129. Gimble J, Guilak F. Adipose-derived adult stem cells: isolation, characterization, and differentiation potential. Cytotherapy. 2003;5:362-9.

130. Gimble JM, Guilak F. Differentiation potential of adipose derived adult stem (ADAS) cells. Curr.Top.Dev.Biol. 2003;58:137-60.

131. Guilak F, Lott KE, Awad HA et al. Clonal analysis of the differentiation potential of human adipose-derived adult stem cells. J.Cell Physiol 2006;206:229-37.

132. Bunnell BA, Flaat $M$, Gagliardi $C$ et al. Adipose-derived stem cells: isolation, expansion and differentiation. Methods 2008;45:115-20.

133. Bray GA. Medical consequences of obesity. J.Clin.Endocrinol.Metab 2004;89:2583-9. 
134. Katz AJ, Llull R, Hedrick MH et al. Emerging approaches to the tissue engineering of fat. Clin.Plast.Surg. 1999;26:587-603, viii.

135. Fraser JK, Wulur I, Alfonso Z et al. Fat tissue: an underappreciated source of stem cells for biotechnology. Trends Biotechnol. 2006;24:150-4.

136. Fraser JK, Zhu M, Wulur I et al. Adipose-derived stem cells. Methods Mol.Biol. 2008;449:59-67.

137. Mizuno $\mathrm{H}$. Adipose-derived stem cells for tissue repair and regeneration: ten years of research and a literature review. J.Nippon Med.Sch 2009;76:56-66.

138. Zuk PA, Zhu M, Ashjian P et al. Human adipose tissue is a source of multipotent stem cells. Mol.Biol.Cell 2002;13:4279-95.

139. De Ugarte DA, Alfonso Z, Zuk PA et al. Differential expression of stem cell mobilization-associated molecules on multi-lineage cells from adipose tissue and bone marrow. Immunol.Lett. 2003;89:267-70.

140. De Ugarte DA, Morizono K, Elbarbary A et al. Comparison of multi-lineage cells from human adipose tissue and bone marrow. Cells Tissues. Organs 2003;174:101-9.

141. Yoshimura $\mathrm{H}$, Muneta $\mathrm{T}$, Nimura A et al. Comparison of rat mesenchymal stem cells derived from bone marrow, synovium, periosteum, adipose tissue, and muscle. Cell Tissue Res. 2007;327:449-62.

142. Zuk PA, Zhu M, Mizuno $\mathrm{H}$ et al. Multilineage cells from human adipose tissue: implications for cell-based therapies. Tissue Eng 2001;7:211-28.

143. Dragoo JL, Samimi B, Zhu M et al. Tissue-engineered cartilage and bone using stem cells from human infrapatellar fat pads. J.Bone Joint Surg.Br. 2003;85:740-7.

144. Halvorsen YD, Franklin D, Bond AL et al. Extracellular matrix mineralization and osteoblast gene expression by human adipose tissue-derived stromal cells. Tissue Eng 2001;7:729-41.

145. Cowan CM, Shi YY, Aalami OO et al. Adipose-derived adult stromal cells heal critical-size mouse calvarial defects. Nat.Biotechnol. 2004;22:560-7.

146. Strem BM, Hicok KC, Zhu M et al. Multipotential differentiation of adipose tissue-derived stem cells. Keio J.Med. 2005;54:132-41.

147. Winter A, Breit S, Parsch D et al. Cartilage-like gene expression in differentiated 
human stem cell spheroids: a comparison of bone marrow-derived and adipose tissue-derived stromal cells. Arthritis Rheum. 2003;48:418-29.

148. Bilgen $B$, Ren $Y$, Pei $M$ et al. CD14-negative isolation enhances chondrogenesis in synovial fibroblasts. Tissue Eng Part A 2009;15:3261-70.

149. Pei M, He F, Vunjak-Novakovic G. Synovium-derived stem cell-based chondrogenesis. Differentiation 2008;76:1044-56.

150. Shirasawa S, Sekiya I, Sakaguchi $Y$ et al. In vitro chondrogenesis of human synovium-derived mesenchymal stem cells: optimal condition and comparison with bone marrow-derived cells. J.Cell Biochem. 2006;97:84-97.

151. Yoo JU, Barthel TS, Nishimura $\mathrm{K}$ et al. The chondrogenic potential of human bone-marrow-derived mesenchymal progenitor cells. J.Bone Joint Surg.Am. 1998;80:1745-57.

152. Sakaguchi $\mathrm{Y}$, Sekiya I, Yagishita $\mathrm{K}$ et al. Comparison of human stem cells derived from various mesenchymal tissues: superiority of synovium as a cell source. Arthritis Rheum. 2005;52:2521-9.

153. Nagase $\mathrm{T}$, Muneta $\mathrm{T}, \mathrm{Ju} \mathrm{YJ}$ et al. Analysis of the chondrogenic potential of human synovial stem cells according to harvest site and culture parameters in knees with medial compartment osteoarthritis. Arthritis Rheum. 2008;58:1389-98.

154. Koga H, Muneta $T$, Nagase $T$ et al. Comparison of mesenchymal tissues-derived stem cells for in vivo chondrogenesis: suitable conditions for cell therapy of cartilage defects in rabbit. Cell Tissue Res. 2008;333:207-15.

155. Pei M, He F, Kish VL et al. Engineering of functional cartilage tissue using stem cells from synovial lining: a preliminary study. Clin.Orthop. Relat Res. 2008;466:1880-9.

156. Ashton BA, Allen TD, Howlett CR et al. Formation of bone and cartilage by marrow stromal cells in diffusion chambers in vivo. Clin.Orthop.Relat Res. 1980;294-307.

157. Koga H, Engebretsen L, Brinchmann JE et al. Mesenchymal stem cell-based therapy for cartilage repair: a review. Knee.Surg.Sports Traumatol.Arthrosc. 2009;17:1289-97.

158. Sekiya I, Colter DC, Prockop DJ. BMP-6 enhances chondrogenesis in a subpopulation of human marrow stromal cells. Biochem.Biophys.Res.Commun. 
2001;284:411-8.

159. Vinatier C, Mrugala D, Jorgensen C et al. Cartilage engineering: a crucial combination of cells, biomaterials and biofactors. Trends Biotechnol. 2009;27:307-14.

160. Lee JW, Kim YH, Kim SH et al. Chondrogenic differentiation of mesenchymal stem cells and its clinical applications. Yonsei Med.J. 2004;45 Suppl:41-7.

161. Grimaud E, Heymann D, Redini F. Recent advances in TGF-beta effects on chondrocyte metabolism. Potential therapeutic roles of TGF-beta in cartilage disorders. Cytokine Growth Factor Rev. 2002;13:241-57.

162. Fan $H, H u ~ Y$, Qin $L$ et al. Porous gelatin-chondroitin-hyaluronate tri-copolymer scaffold containing microspheres loaded with TGF-beta1 induces differentiation of mesenchymal stem cells in vivo for enhancing cartilage repair. J.Biomed.Mater.Res.A 2006;77:785-94.

163. Sekiya I, Larson BL, Vuoristo JT et al. Comparison of effect of BMP-2, -4, and -6 on in vitro cartilage formation of human adult stem cells from bone marrow stroma. Cell Tissue Res. 2005;320:269-76.

164. Grunder T, Gaissmaier C, Fritz J et al. Bone morphogenetic protein (BMP)-2 enhances the expression of type II collagen and aggrecan in chondrocytes embedded in alginate beads. Osteoarthritis. Cartilage. 2004;12:559-67.

165. Hennig $\mathrm{T}$, Lorenz $\mathrm{H}$, Thiel $\mathrm{A}$ et al. Reduced chondrogenic potential of adipose tissue derived stromal cells correlates with an altered TGFbeta receptor and BMP profile and is overcome by BMP-6. J.Cell Physiol 2007;211:682-91.

166. Dupont J, Holzenberger M. Biology of insulin-like growth factors in development. Birth Defects Res.C.Embryo.Today 2003;69:257-71.

167. Schmidt MB, Chen EH, Lynch SE. A review of the effects of insulin-like growth factor and platelet derived growth factor on in vivo cartilage healing and repair. Osteoarthritis. Cartilage. 2006;14:403-12.

168. Ornitz DM, Marie PJ. FGF signaling pathways in endochondral and intramembranous bone development and human genetic disease. Genes Dev. 2002;16:1446-65. 
169. Dailey L, Ambrosetti D, Mansukhani A et al. Mechanisms underlying differential responses to FGF signaling. Cytokine Growth Factor Rev. 2005;16:233-47.

170. Martin I, Vunjak-Novakovic G, Yang J et al. Mammalian chondrocytes expanded in the presence of fibroblast growth factor 2 maintain the ability to differentiate and regenerate three-dimensional cartilaginous tissue. Exp.Cell Res. 1999;253:681-8.

171. Ishii I, Mizuta H, Sei A et al. Healing of full-thickness defects of the articular cartilage in rabbits using fibroblast growth factor-2 and a fibrin sealant. J.Bone Joint Surg.Br. 2007;89:693-700.

172. Mackay AM, Beck SC, Murphy JM et al. Chondrogenic differentiation of cultured human mesenchymal stem cells from marrow. Tissue Eng 1998;4:415-28.

173. Farquharson C, Berry JL, Mawer EB et al. Ascorbic acid-induced chondrocyte terminal differentiation: the role of the extracellular matrix and 1,25-dihydroxyvitamin D. Eur.J.Cell Biol. 1998;76:110-8.

174. Heng BC, Cao T, Lee EH. Directing stem cell differentiation into the chondrogenic lineage in vitro. Stem Cells 2004;22:1152-67.

175. SALMON WD, Jr. Importance of amino acids in the actions of insulin and serum sulfation factor to stimulate sulfate uptake by cartilage from hypophysectomized rats. J.Lab Clin.Med. 1960;56:673-81.

176. Yamaoka $\mathrm{H}$, Asato $\mathrm{H}$, Ogasawara $\mathrm{T}$ et al. Cartilage tissue engineering using human auricular chondrocytes embedded in different hydrogel materials. J.Biomed.Mater.Res.A 2006;78:1-11.

177. Schipani E. Hypoxia and HIF-1 alpha in chondrogenesis. Semin.Cell Dev.Biol. 2005;16:539-46.

178. Robins JC, Akeno N, Mukherjee A et al. Hypoxia induces chondrocyte-specific gene expression in mesenchymal cells in association with transcriptional activation of Sox9. Bone 2005;37:313-22.

179. $\mathrm{Xu} \mathrm{Y,} \mathrm{Malladi} \mathrm{P}$, Chiou $\mathrm{M}$ et al. In vitro expansion of adipose-derived adult stromal cells in hypoxia enhances early chondrogenesis. Tissue Eng 2007;13:2981-93.

180. Sun $\mathrm{X}$, Wei $\mathrm{Y}$. The role of hypoxia-inducible factor in osteogenesis and chondrogenesis. Cytotherapy. 2009;11:261-7. 
181. Duval E, Leclercq S, Elissalde JM et al. Hypoxia-inducible factor 1alpha inhibits the fibroblast-like markers type I and type III collagen during hypoxia-induced chondrocyte redifferentiation: hypoxia not only induces type II collagen and aggrecan, but it also inhibits type I and type III collagen in the hypoxia-inducible factor 1alpha-dependent redifferentiation of chondrocytes. Arthritis Rheum. 2009;60:3038-48.

182. Domm C, Schunke $M$, Christesen $\mathrm{K}$ et al. Redifferentiation of dedifferentiated bovine articular chondrocytes in alginate culture under low oxygen tension. Osteoarthritis. Cartilage. 2002;10:13-22.

183. Murphy $\mathrm{CL}$, Sambanis A. Effect of oxygen tension and alginate encapsulation on restoration of the differentiated phenotype of passaged chondrocytes. Tissue Eng 2001;7:791-803.

184. Pelttari K, Winter A, Steck E et al. Premature induction of hypertrophy during in vitro chondrogenesis of human mesenchymal stem cells correlates with calcification and vascular invasion after ectopic transplantation in SCID mice. Arthritis Rheum. 2006;54:3254-66.

185. Pelttari K, Steck E, Richter W. The use of mesenchymal stem cells for chondrogenesis. Injury 2008;39 Suppl 1:S58-S65.

186. Cancedda R, Castagnola P, Cancedda FD et al. Developmental control of chondrogenesis and osteogenesis. Int.J.Dev.Biol. 2000;44:707-14.

187. Morita K, Miyamoto T, Fujita N et al. Reactive oxygen species induce chondrocyte hypertrophy in endochondral ossification. J.Exp.Med. 2007;204:1613-23.

188. Steinert AF, Proffen B, Kunz M et al. Hypertrophy is induced during the in vitro chondrogenic differentiation of human mesenchymal stem cells by bone morphogenetic protein-2 and bone morphogenetic protein-4 gene transfer. Arthritis Res.Ther. 2009;11:R148.

189. Zhang X, Ziran N, Goater JJ et al. Primary murine limb bud mesenchymal cells in long-term culture complete chondrocyte differentiation: TGF-beta delays hypertrophy and PGE2 inhibits terminal differentiation. Bone 2004;34:809-17.

190. Zheng Q, Zhou G, Morello $\mathrm{R}$ et al. Type X collagen gene regulation by Runx2 
contributes directly to its hypertrophic chondrocyte-specific expression in vivo. J.Cell Biol. 2003;162:833-42.

191. Weiss S, Hennig T, Bock R et al. Impact of growth factors and PTHrP on early and late chondrogenic differentiation of human mesenchymal stem cells. J.Cell Physiol 2010;223:84-93.

192. Fischer J, Dickhut A, Rickert M et al. Articular chondrocytes secrete PTHrP and inhibit hypertrophy of mesenchymal stem cells in coculture during chondrogenesis. Arthritis Rheum. 2010.

193. Heldin $\mathrm{CH}$, Miyazono $\mathrm{K}$, ten DP. TGF-beta signalling from cell membrane to nucleus through SMAD proteins. Nature 1997;390:465-71.

194. Miyazawa K, Shinozaki M, Hara T et al. Two major Smad pathways in TGF-beta superfamily signalling. Genes Cells 2002;7:1191-204.

195. Miyazono K, ten DP, Heldin CH. TGF-beta signaling by Smad proteins. Adv. Immunol. 2000;75:115-57.

196. Shimizu A, Kato M, Nakao A et al. Identification of receptors and Smad proteins involved in activin signalling in a human epidermal keratinocyte cell line. Genes Cells 1998;3:125-34.

197. Wrana JL, Attisano L, Wieser $\mathrm{R}$ et al. Mechanism of activation of the TGF-beta receptor. Nature 1994;370:341-7.

198. Attisano L, Wrana JL. Mads and Smads in TGF beta signalling. Curr. Opin.Cell Biol. 1998;10:188-94.

199. Chen YG, Liu F, Massague J. Mechanism of TGFbeta receptor inhibition by FKBP12. EMBO J. 1997;16:3866-76.

200. Tamaki K, Souchelnytskyi S, Itoh S et al. Intracellular signaling of osteogenic protein-1 through Smad5 activation. J.Cell Physiol 1998;177:355-63.

201. Schlessinger J. Cell signaling by receptor tyrosine kinases. Cell 2000;103:211-25.

202. McKay MM, Morrison DK. Integrating signals from RTKs to ERK/MAPK. Oncogene 2007;26:3113-21.

203. Ravichandran KS. Signaling via Shc family adapter proteins. Oncogene 2001;20:6322-30 
204. Zhang YE. Non-Smad pathways in TGF-beta signaling. Cell Res. 2009;19:128-39.

205. Watanabe $\mathrm{H}$, de Caestecker MP, Yamada Y. Transcriptional cross-talk between Smad, ERK1/2, and p38 mitogen-activated protein kinase pathways regulates transforming growth factor-beta-induced aggrecan gene expression in chondrogenic ATDC5 cells. J.Biol.Chem. 2001;276:14466-73.

206. Li J, Zhao Z, Liu J et al. MEK/ERK and p38 MAPK regulate chondrogenesis of rat bone marrow mesenchymal stem cells through delicate interaction with TGF-beta1/Smads pathway. Cell Prolif. 2010;43:333-43.

207. Bobick BE, Kulyk WM. The MEK-ERK signaling pathway is a negative regulator of cartilage-specific gene expression in embryonic limb mesenchyme. J.Biol.Chem. 2004;279:4588-95.

208. Kim HJ, Im GI. The effects of ERK1/2 inhibitor on the chondrogenesis of bone marrow- and adipose tissue-derived multipotent mesenchymal stromal cells. Tissue Eng Part A 2010;16:851-60.

209. Weston CR, Davis RJ. The JNK signal transduction pathway. Curr.Opin.Cell Biol. 2007;19:142-9.

210. Frey RS, Mulder KM. Involvement of extracellular signal-regulated kinase 2 and stress-activated protein kinase/Jun N-terminal kinase activation by transforming growth factor beta in the negative growth control of breast cancer cells. Cancer Res. 1997;57:628-33.

211. Hanafusa $\mathrm{H}$, Ninomiya-Tsuji $\mathrm{J}$, Masuyama $\mathrm{N}$ et al. Involvement of the p38 mitogen-activated protein kinase pathway in transforming growth factor-beta-induced gene expression. J.Biol.Chem. 1999;274:27161-7.

212. Bhowmick NA, Zent R, Ghiassi $M$ et al. Integrin beta 1 signaling is necessary for transforming growth factor-beta activation of p38MAPK and epithelial plasticity. J.Biol.Chem. 2001;276:46707-13.

213. Yu L, Hebert MC, Zhang YE. TGF-beta receptor-activated p38 MAP kinase mediates Smad-independent TGF-beta responses. EMBO J. 2002;21:3749-59.

214. Engel ME, McDonnell MA, Law BK et al. Interdependent SMAD and JNK signaling in transforming growth factor-beta-mediated transcription. J.Biol.Chem. 
1999;274:37413-20.

215. Hocevar BA, Brown TL, Howe PH. TGF-beta induces fibronectin synthesis through a c-Jun N-terminal kinase-dependent, Smad4-independent pathway. EMBO J. 1999;18:1345-56.

216. Wilkes MC, Mitchell H, Penheiter SG et al. Transforming growth factor-beta activation of phosphatidylinositol 3-kinase is independent of Smad2 and Smad3 and regulates fibroblast responses via p21-activated kinase-2. Cancer Res. 2005;65:10431-40.

217. Bakin AV, Tomlinson AK, Bhowmick NA et al. Phosphatidylinositol 3-kinase function is required for transforming growth factor beta-mediated epithelial to mesenchymal transition and cell migration. J.Biol.Chem. 2000;275:36803-10.

218. Chen $\mathrm{RH}$, Su $\mathrm{YH}$, Chuang $\mathrm{RL}$ et al. Suppression of transforming growth factor-beta-induced apoptosis through a phosphatidylinositol 3-kinase/Akt-dependent pathway. Oncogene 1998;17:1959-68.

219. Song $\mathrm{K}$, Wang $\mathrm{H}$, Krebs $\mathrm{TL}$ et al. Novel roles of Akt and mTOR in suppressing TGF-beta/ALK5-mediated Smad3 activation. EMBO J. 2006;25:58-69.

220. Conery AR, Cao Y, Thompson EA et al. Akt interacts directly with Smad3 to regulate the sensitivity to TGF-beta induced apoptosis. Nat.Cell Biol. 2004;6:366-72.

221. Remy I, Montmarquette A, Michnick SW. PKB/Akt modulates TGF-beta signalling through a direct interaction with Smad3. Nat.Cell Biol. 2004;6:358-65.

222. Edlund S, Landstrom M, Heldin $\mathrm{CH}$ et al. Transforming growth factor-beta-induced mobilization of actin cytoskeleton requires signaling by small GTPases Cdc42 and RhoA. Mol.Biol.Cell 2002;13:902-14.

223. Jaffe AB, Hall A. Rho GTPases: biochemistry and biology. Annu.Rev.Cell Dev.Biol. 2005;21:247-69.

224. Bhowmick NA, Ghiassi M, Bakin A et al. Transforming growth factor-beta1 mediates epithelial to mesenchymal transdifferentiation through a RhoA-dependent mechanism. Mol.Biol.Cell 2001;12:27-36.

225. Wilkes MC, Murphy SJ, Garamszegi N et al. Cell-type-specific activation of PAK2 by transforming growth factor beta independent of Smad2 and Smad3. Mol.Cell Biol. 
2003;23:8878-89.

226. Edlund S, Lee SY, Grimsby S et al. Interaction between Smad7 and beta-catenin: importance for transforming growth factor beta-induced apoptosis. Mol.Cell Biol. 2005;25:1475-88.

227. Edlund S, Landstrom M, Heldin $\mathrm{CH}$ et al. Smad7 is required for TGF-beta-induced activation of the small GTPase Cdc42. J.Cell Sci. 2004;117:1835-47.

228. Akiyama H. Control of chondrogenesis by the transcription factor Sox9. Mod.Rheumatol. 2008;18:213-9.

229. Ng LJ, Wheatley S, Muscat GE et al. SOX9 binds DNA, activates transcription, and coexpresses with type II collagen during chondrogenesis in the mouse. Dev.Biol. $1997 ; 183: 108-21$.

230. Zhao Q, Eberspaecher H, Lefebvre $V$ et al. Parallel expression of Sox9 and Col2a1 in cells undergoing chondrogenesis. Dev.Dyn. 1997;209:377-86.

231. Akiyama $\mathrm{H}$, Chaboissier MC, Martin JF et al. The transcription factor Sox9 has essential roles in successive steps of the chondrocyte differentiation pathway and is required for expression of Sox5 and Sox6. Genes Dev. 2002;16:2813-28.

232. Bi W, Deng JM, Zhang $Z$ et al. Sox9 is required for cartilage formation. Nat.Genet. 1999;22:85-9.

233. Mori-Akiyama $\mathrm{Y}$, van den $\mathrm{BM}$, van $\mathrm{Es} \mathrm{JH}$ et al. SOX9 is required for the differentiation of paneth cells in the intestinal epithelium. Gastroenterology 2007;133:539-46.

234. Mori-Akiyama $\mathrm{Y}$, Akiyama $\mathrm{H}$, Rowitch $\mathrm{DH}$ et al. Sox9 is required for determination of the chondrogenic cell lineage in the cranial neural crest. Proc.Natl.Acad.Sci.U.S.A 2003;100:9360-5.

235. Zhang G, Miyamoto MM, Cohn MJ. Lamprey type II collagen and Sox9 reveal an ancient origin of the vertebrate collagenous skeleton. Proc.Natl.Acad.Sci.U.S.A 2006;103:3180-5.

236. Zhou $G$, Zheng Q, Engin $F$ et al. Dominance of SOX9 function over RUNX2 during skeletogenesis. Proc.Natl.Acad.Sci.U.S.A 2006;103:19004-9.

237. Laurencin $\mathrm{CT}$, Ambrosio AM, Borden MD et al. Tissue engineering: orthopedic 
applications. Annu.Rev.Biomed.Eng 1999;1:19-46.

238. Hoque ME, Hutmacher DW, Feng $W$ et al. Fabrication using a rapid prototyping system and in vitro characterization of PEG-PCL-PLA scaffolds for tissue engineering. J.Biomater.Sci.Polym.Ed 2005;16:1595-610.

239. Almarza AJ, Athanasiou KA. Effects of initial cell seeding density for the tissue engineering of the temporomandibular joint disc. Ann.Biomed.Eng 2005;33:943-50.

240. Griffon DJ, Sedighi MR, Sendemir-Urkmez A et al. Evaluation of vacuum and dynamic cell seeding of polyglycolic acid and chitosan scaffolds for cartilage engineering. Am.J.Vet.Res. 2005;66:599-605.

241. Hunter CJ, Levenston ME. Maturation and integration of tissue-engineered cartilages within an in vitro defect repair model. Tissue Eng 2004;10:736-46.

242. Mahmoudifar N, Doran PM. Tissue engineering of human cartilage in bioreactors using single and composite cell-seeded scaffolds. Biotechnol.Bioeng. 2005;91:338-55.

243. Mouw JK, Case ND, Guldberg RE et al. Variations in matrix composition and GAG fine structure among scaffolds for cartilage tissue engineering. Osteoarthritis. Cartilage. 2005;13:828-36.

244. Uematsu K, Hattori K, Ishimoto $\mathrm{Y}$ et al. Cartilage regeneration using mesenchymal stem cells and a three-dimensional poly-lactic-glycolic acid (PLGA) scaffold. Biomaterials 2005;26:4273-9.

245. Li WJ, Danielson KG, Alexander PG et al. Biological response of chondrocytes cultured in three-dimensional nanofibrous poly(epsilon-caprolactone) scaffolds. J.Biomed.Mater.Res.A 2003;67:1105-14.

246. Malda J, Woodfield TB, van d, V et al. The effect of PEGT/PBT scaffold architecture on the composition of tissue engineered cartilage. Biomaterials 2005;26:63-72.

247. Miot S, Woodfield T, Daniels AU et al. Effects of scaffold composition and architecture on human nasal chondrocyte redifferentiation and cartilaginous matrix deposition. Biomaterials 2005;26:2479-89.

248. Xia W, Liu W, Cui $L$ et al. Tissue engineering of cartilage with the use of chitosan-gelatin complex scaffolds. J.Biomed.Mater.Res.B Appl.Biomater. 
$2004 ; 71: 373-80$.

249. Yamane S, Iwasaki N, Majima T et al. Feasibility of chitosan-based hyaluronic acid hybrid biomaterial for a novel scaffold in cartilage tissue engineering. Biomaterials 2005;26:611-9.

250. Iwasaki N, Yamane ST, Majima T et al. Feasibility of polysaccharide hybrid materials for scaffolds in cartilage tissue engineering: evaluation of chondrocyte adhesion to polyion complex fibers prepared from alginate and chitosan. Biomacromolecules. 2004;5:828-33.

251. Nettles DL, Vail TP, Morgan MT et al. Photocrosslinkable hyaluronan as a scaffold for articular cartilage repair. Ann.Biomed.Eng 2004;32:391-7.

252. Burdick JA, Chung $\mathrm{C}$, Jia $X$ et al. Controlled degradation and mechanical behavior of photopolymerized hyaluronic acid networks. Biomacromolecules. 2005;6:386-91.

253. Li Q, Williams CG, Sun DD et al. Photocrosslinkable polysaccharides based on chondroitin sulfate. J.Biomed.Mater.Res.A 2004;68:28-33.

254. Bryant SJ, Anseth KS, Lee DA et al. Crosslinking density influences the morphology of chondrocytes photoencapsulated in PEG hydrogels during the application of compressive strain. J.Orthop.Res. 2004;22:1143-9.

255. Park H, Temenoff JS, Holland TA et al. Delivery of TGF-beta1 and chondrocytes via injectable, biodegradable hydrogels for cartilage tissue engineering applications. Biomaterials 2005;26:7095-103.

256. Holland TA, Tabata Y, Mikos AG. Dual growth factor delivery from degradable oligo(poly(ethylene glycol) fumarate) hydrogel scaffolds for cartilage tissue engineering. J.Control Release 2005;101:111-25.

257. Vunjak-Novakovic G, Meinel L, Altman G et al. Bioreactor cultivation of osteochondral grafts. Orthod.Craniofac.Res. 2005;8:209-18.

258. Tognana E, Chen F, Padera RF et al. Adjacent tissues (cartilage, bone) affect the functional integration of engineered calf cartilage in vitro. Osteoarthritis. Cartilage. 2005;13:129-38.

259. Kuo CK, Li WJ, Mauck RL et al. Cartilage tissue engineering: its potential and uses. Curr.Opin.Rheumatol. 2006;18:64-73. 
260. Buschmann MD, Gluzband YA, Grodzinsky AJ et al. Mechanical compression modulates matrix biosynthesis in chondrocyte/agarose culture. J.Cell Sci. 1995;108 ( Pt 4):1497-508.

261. Hung CT, Mauck RL, Wang CC et al. A paradigm for functional tissue engineering of articular cartilage via applied physiologic deformational loading. Ann.Biomed.Eng 2004;32:35-49.

262. Smith RL, Carter DR, Schurman DJ. Pressure and shear differentially alter human articular chondrocyte metabolism: a review. Clin.Orthop.Relat Res. 2004;S89-S95.

263. Pei M, He F, Boyce BM et al. Repair of full-thickness femoral condyle cartilage defects using allogeneic synovial cell-engineered tissue constructs. Osteoarthritis. Cartilage. 2009;17:714-22.

264. Cukierman E, Pankov R, Yamada KM. Cell interactions with three-dimensional matrices. Curr. Opin. Cell Biol. 2002;14:633-9.

265. Gordon MY. Extracellular matrix of the marrow microenvironment. Br.J.Haematol. 1988;70:1-4.

266. Lin H. The stem-cell niche theory: lessons from flies. Nat.Rev.Genet. 2002;3:931-40.

267. Moore KA, Lemischka IR. Stem cells and their niches. Science 2006;311:1880-5.

268. Watt FM, Hogan BL. Out of Eden: stem cells and their niches. Science 2000;287:1427-30.

269. Hamilton R, Campbell FR. Immunochemical localization of extracellular materials in bone marrow of rats. Anat.Rec. 1991;231:218-24.

270. Hocking AM, Shinomura T, McQuillan DJ. Leucine-rich repeat glycoproteins of the extracellular matrix. Matrix Biol. 1998;17:1-19.

271. Chen XD. Extracellular matrix provides an optimal niche for the maintenance and propagation of mesenchymal stem cells. Birth Defects Res.C.Embryo.Today 2010;90:45-54.

272. Robey PG, Fedarko NS, Hefferan TE et al. Structure and molecular regulation of bone matrix proteins. J.Bone Miner.Res. 1993;8 Suppl 2:S483-S487.

273. Delany AM, Canalis E. The metastasis-associated metalloproteinase stromelysin-3 
is induced by transforming growth factor-beta in osteoblasts and fibroblasts. Endocrinology 2001;142:1561-6.

274. Ruoslahti E, Yamaguchi Y. Proteoglycans as modulators of growth factor activities. Cell 1991;64:867-9.

275. Ruoslahti E, Yamaguchi $Y$, Hildebrand A et al. Extracellular matrix/growth factor interactions. Cold Spring Harb.Symp.Quant.Biol. 1992;57:309-15.

276. Bianco P, Riminucci M, Gronthos S et al. Bone marrow stromal stem cells: nature, biology, and potential applications. Stem Cells 2001;19:180-92.

277. Katayama Y, Battista M, Kao WM et al. Signals from the sympathetic nervous system regulate hematopoietic stem cell egress from bone marrow. Cell 2006;124:407-21.

278. Maroudas A, Bayliss MT, Venn MF. Further studies on the composition of human femoral head cartilage. Ann.Rheum.Dis. 1980;39:514-23.

279. Werb Z, Chin JR. Extracellular matrix remodeling during morphogenesis. Ann.N.Y.Acad.Sci. 1998;857:110-8.

280. Boudreau NJ, Jones PL. Extracellular matrix and integrin signalling: the shape of things to come. Biochem.J. 1999;339 ( Pt 3):481-8.

281. Docheva D, Popov C, Mutschler W et al. Human mesenchymal stem cells in contact with their environment: surface characteristics and the integrin system. J.Cell Mol.Med. 2007;11:21-38.

282. Giancotti FG, Ruoslahti E. Integrin signaling. Science 1999;285:1028-32.

283. Calderwood DA. Integrin activation. J. Cell Sci. 2004;117:657-66.

284. Fisher LW, Torchia DA, Fohr B et al. Flexible structures of SIBLING proteins, bone sialoprotein, and osteopontin. Biochem.Biophys. Res.Commun. 2001;280:460-5.

285. Burridge K, Chrzanowska-Wodnicka M. Focal adhesions, contractility, and signaling. Annu.Rev.Cell Dev.Biol. 1996;12:463-518.

286. Schaller MD, Parsons JT. Focal adhesion kinase and associated proteins. Curr.Opin. Cell Biol. 1994;6:705-10.

287. Schlaepfer DD, Hanks SK, Hunter T et al. Integrin-mediated signal transduction linked to Ras pathway by GRB2 binding to focal adhesion kinase. Nature 
$1994 ; 372: 786-91$.

288. Chen Q, Kinch MS, Lin TH et al. Integrin-mediated cell adhesion activates mitogen-activated protein kinases. J.Biol.Chem. 1994;269:26602-5.

289. Chevalier X. Fibronectin, cartilage, and osteoarthritis. Semin.Arthritis Rheum. 1993;22:307-18.

290. Hayashi T, Abe E, Jasin HE. Fibronectin synthesis in superficial and deep layers of normal articular cartilage. Arthritis Rheum. 1996;39:567-73.

291. Pytela R, Pierschbacher MD, Ruoslahti E. Identification and isolation of a $140 \mathrm{kd}$ cell surface glycoprotein with properties expected of a fibronectin receptor. Cell 1985;40:191-8.

292. Heino J, Kapyla J. Cellular receptors of extracellular matrix molecules. Curr.Pharm.Des 2009;15:1309-17.

293. Hynes RO. Integrins: bidirectional, allosteric signaling machines. Cell 2002;110:673-87.

294. Pierschbacher MD, Hayman EG, Ruoslahti E. The cell attachment determinant in fibronectin. J.Cell Biochem. 1985;28:115-26.

295. Pierschbacher MD, Ruoslahti E. Cell attachment activity of fibronectin can be duplicated by small synthetic fragments of the molecule. Nature 1984;309:30-3.

296. Lane NE, Yao W, Nakamura MC et al. Mice lacking the integrin beta5 subunit have accelerated osteoclast maturation and increased activity in the estrogen-deficient state. J.Bone Miner.Res. 2005;20:58-66.

297. Munger JS, Huang $\mathrm{X}$, Kawakatsu $\mathrm{H}$ et al. The integrin alpha $\vee$ beta 6 binds and activates latent TGF beta 1: a mechanism for regulating pulmonary inflammation and fibrosis. Cell 1999;96:319-28.

298. Zhu J, Motejlek K, Wang D et al. beta8 integrins are required for vascular morphogenesis in mouse embryos. Development 2002;129:2891-903.

299. Keum E, Kim Y, Kim J et al. Syndecan-4 regulates localization, activity and stability of protein kinase C-alpha. Biochem.J. 2004;378:1007-14.

300. Beauvais DM, Burbach BJ, Rapraeger AC. The syndecan-1 ectodomain regulates alphavbeta3 integrin activity in human mammary carcinoma cells. J.Cell Biol. 
2004;167:171-81.

301. McQuade KJ, Beauvais DM, Burbach BJ et al. Syndecan-1 regulates alphavbeta5 integrin activity in B82L fibroblasts. J.Cell Sci. 2006;119:2445-56.

302. Bloom L, Ingham KC, Hynes RO. Fibronectin regulates assembly of actin filaments and focal contacts in cultured cells via the heparin-binding site in repeat III13. Mol.Biol.Cell 1999;10:1521-36.

303. Morgan MR, Humphries MJ, Bass MD. Synergistic control of cell adhesion by integrins and syndecans. Nat.Rev.Mol.Cell Biol. 2007;8:957-69.

304. Emsley J, Cruz M, Handin R et al. Crystal structure of the von Willebrand Factor A1 domain and implications for the binding of platelet glycoprotein Ib. J.Biol.Chem. 1998;273:10396-401.

305. Voigt S, Gossrau R, Baum O et al. Distribution and quantification of alpha 1-integrin subunit in rat organs. Histochem.J. 1995;27:123-32.

306. Kern A, Eble J, Golbik R et al. Interaction of type IV collagen with the isolated integrins alpha 1 beta 1 and alpha 2 beta 1. Eur.J.Biochem. 1993;215:151-9.

307. Tulla M, Pentikainen OT, Viitasalo T et al. Selective binding of collagen subtypes by integrin alpha $1 \mathrm{l}$, alpha $2 \mathrm{l}$, and alpha $10 \mathrm{l}$ domains. J.Biol.Chem. 2001;276:48206-12.

308. Zutter MM, Santoro SA. Widespread histologic distribution of the alpha 2 beta 1 integrin cell-surface collagen receptor. Am.J.Pathol. 1990;137:113-20.

309. Jokinen J, Dadu E, Nykvist P et al. Integrin-mediated cell adhesion to type I collagen fibrils. J.Biol.Chem. 2004;279:31956-63.

310. Kapyla J, Jaalinoja J, Tulla $\mathrm{M}$ et al. The fibril-associated collagen IX provides a novel mechanism for cell adhesion to cartilaginous matrix. J.Biol.Chem. 2004;279:51677-87.

311. Bengtsson T, Aszodi A, Nicolae $\mathrm{C}$ et al. Loss of alpha10beta1 integrin expression leads to moderate dysfunction of growth plate chondrocytes. J.Cell Sci. 2005;118:929-36.

312. Popova SN, Barczyk M, Tiger CF et al. Alpha11 beta1 integrin-dependent regulation of periodontal ligament function in the erupting mouse incisor. Mol.Cell Biol. 
2007;27:4306-16.

313. Vuoriluoto $\mathrm{K}$, Jokinen $\mathrm{J}$, Kallio $\mathrm{K}$ et al. Syndecan-1 supports integrin alpha2beta1-mediated adhesion to collagen. Exp. Cell Res. 2008;314:3369-81.

314. Hozumi K, Suzuki N, Nielsen PK et al. Laminin alpha1 chain LG4 module promotes cell attachment through syndecans and cell spreading through integrin alpha2beta1. J.Biol.Chem. 2006;281:32929-40.

315. Vogel W, Gish GD, Alves F et al. The discoidin domain receptor tyrosine kinases are activated by collagen. Mol.Cell 1997;1:13-23.

316. Shrivastava A, Radziejewski C, Campbell E et al. An orphan receptor tyrosine kinase family whose members serve as nonintegrin collagen receptors. Mol.Cell 1997;1:25-34.

317. Auger JM, Kuijpers MJ, Senis YA et al. Adhesion of human and mouse platelets to collagen under shear: a unifying model. FASEB J. 2005;19:825-7.

318. Kreidberg JA, Donovan MJ, Goldstein SL et al. Alpha 3 beta 1 integrin has a crucial role in kidney and lung organogenesis. Development 1996;122:3537-47.

319. DiPersio CM, Hodivala-Dilke KM, Jaenisch $\mathrm{R}$ et al. alpha3beta1 Integrin is required for normal development of the epidermal basement membrane. J.Cell Biol. 1997;137:729-42.

320. Mayer U, Saher G, Fassler R et al. Absence of integrin alpha 7 causes a novel form of muscular dystrophy. Nat.Genet. 1997;17:318-23.

321. Stepp MA, Spurr-Michaud S, Tisdale A et al. Alpha 6 beta 4 integrin heterodimer is a component of hemidesmosomes. Proc.Natl.Acad.Sci.U.S.A 1990;87:8970-4.

322. Georges-Labouesse E, Messaddeq N, Yehia G et al. Absence of integrin alpha 6 leads to epidermolysis bullosa and neonatal death in mice. Nat.Genet. 1996;13:370-3.

323. Ibraghimov-Beskrovnaya O, Ervasti JM, Leveille CJ et al. Primary structure of dystrophin-associated glycoproteins linking dystrophin to the extracellular matrix. Nature 1992;355:696-702.

324. Haenggi T, Fritschy JM. Role of dystrophin and utrophin for assembly and function of the dystrophin glycoprotein complex in non-muscle tissue. Cell Mol.Life Sci. 
2006;63:1614-31.

325. Cukierman E, Pankov R, Stevens DR et al. Taking cell-matrix adhesions to the third dimension. Science 2001;294:1708-12.

326. Green JA, Yamada KM. Three-dimensional microenvironments modulate fibroblast signaling responses. Adv.Drug Deliv.Rev. 2007;59:1293-8.

327. Elsdale T, Bard J. Collagen substrata for studies on cell behavior. J.Cell Biol. 1972;54:626-37.

328. Friedl P, Brocker EB. The biology of cell locomotion within three-dimensional extracellular matrix. Cell Mol.Life Sci. 2000;57:41-64.

329. Damianova R, Stefanova N, Cukierman E et al. Three-dimensional matrix induces sustained activation of ERK1/2 via Src/Ras/Raf signaling pathway. Cell Biol.Int. 2008;32:229-34.

330. Pankov R, Endo $\mathrm{Y}$, Even-Ram $\mathrm{S}$ et al. A Rac switch regulates random versus directionally persistent cell migration. J. Cell Biol. 2005;170:793-802.

331. Even-Ram S, Yamada KM. Cell migration in 3D matrix. Curr.Opin.Cell Biol. 2005;17:524-32.

332. Kuhn NZ, Tuan RS. Regulation of stemness and stem cell niche of mesenchymal stem cells: implications in tumorigenesis and metastasis. J.Cell Physiol 2010;222:268-77.

333. Gang EJ, Bosnakovski D, Figueiredo CA et al. SSEA-4 identifies mesenchymal stem cells from bone marrow. Blood 2007;109:1743-51.

334. Minguell JJ, Erices A, Conget P. Mesenchymal stem cells. Exp.Biol.Med.(Maywood.) 2001;226:507-20.

335. Majd H, Wipff PJ, Buscemi L et al. A novel method of dynamic culture surface expansion improves mesenchymal stem cell proliferation and phenotype. Stem Cells 2009;27:200-9.

336. Javazon EH, Beggs KJ, Flake AW. Mesenchymal stem cells: paradoxes of passaging. Exp.Hematol. 2004;32:414-25.

337. Vacanti V, Kong E, Suzuki G et al. Phenotypic changes of adult porcine mesenchymal stem cells induced by prolonged passaging in culture. J.Cell Physiol 
2005;205:194-201.

338. Bruder SP, Jaiswal N, Haynesworth SE. Growth kinetics, self-renewal, and the osteogenic potential of purified human mesenchymal stem cells during extensive subcultivation and following cryopreservation. J.Cell Biochem. 1997;64:278-94.

339. Lai Y, Sun Y, Skinner CM et al. Reconstitution of Marrow-derived Extracellular Matrix Ex Vivo: a Robust Culture System for Expanding Large-scale Highly Functional Human Mesenchymal Stem Cells. Stem Cells Dev. 2009.

340. Song L, Webb NE, Song $Y$ et al. Identification and functional analysis of candidate genes regulating mesenchymal stem cell self-renewal and multipotency. Stem Cells 2006;24:1707-18. 
Figure 1.1

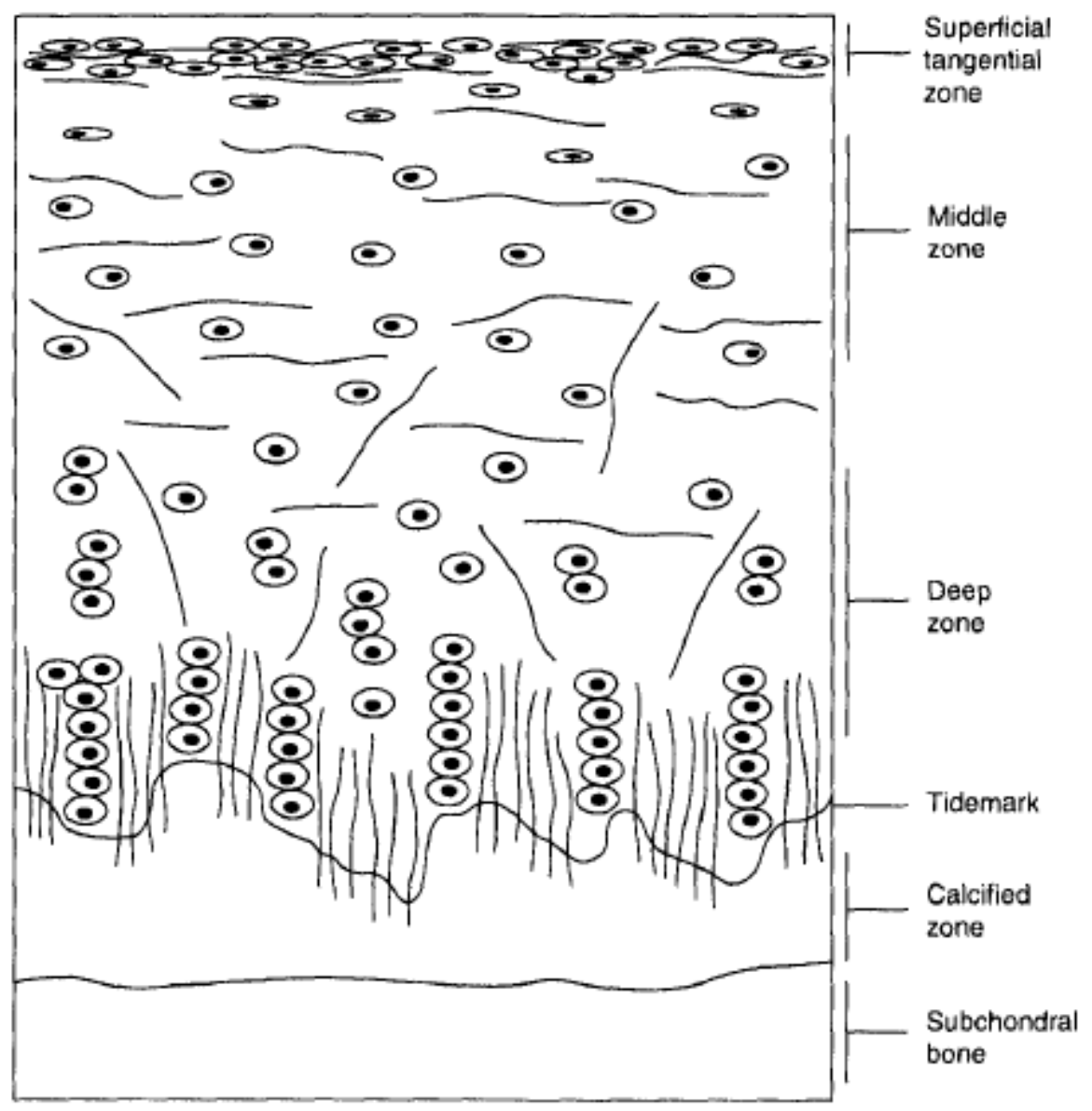

Figure 1.1 The structure of articular cartilage is composed by four zones: the superficial zone, the middle zone, the deep zone and the calcified cartilage zone. Figures are from Nelson $L$, Fairclough J, Archer CW. Use of stem cells in the biological repair of articular cartilage. Expert.Opin.Biol.Ther. 2010;10:43-55. 


\section{Figure 1.2}
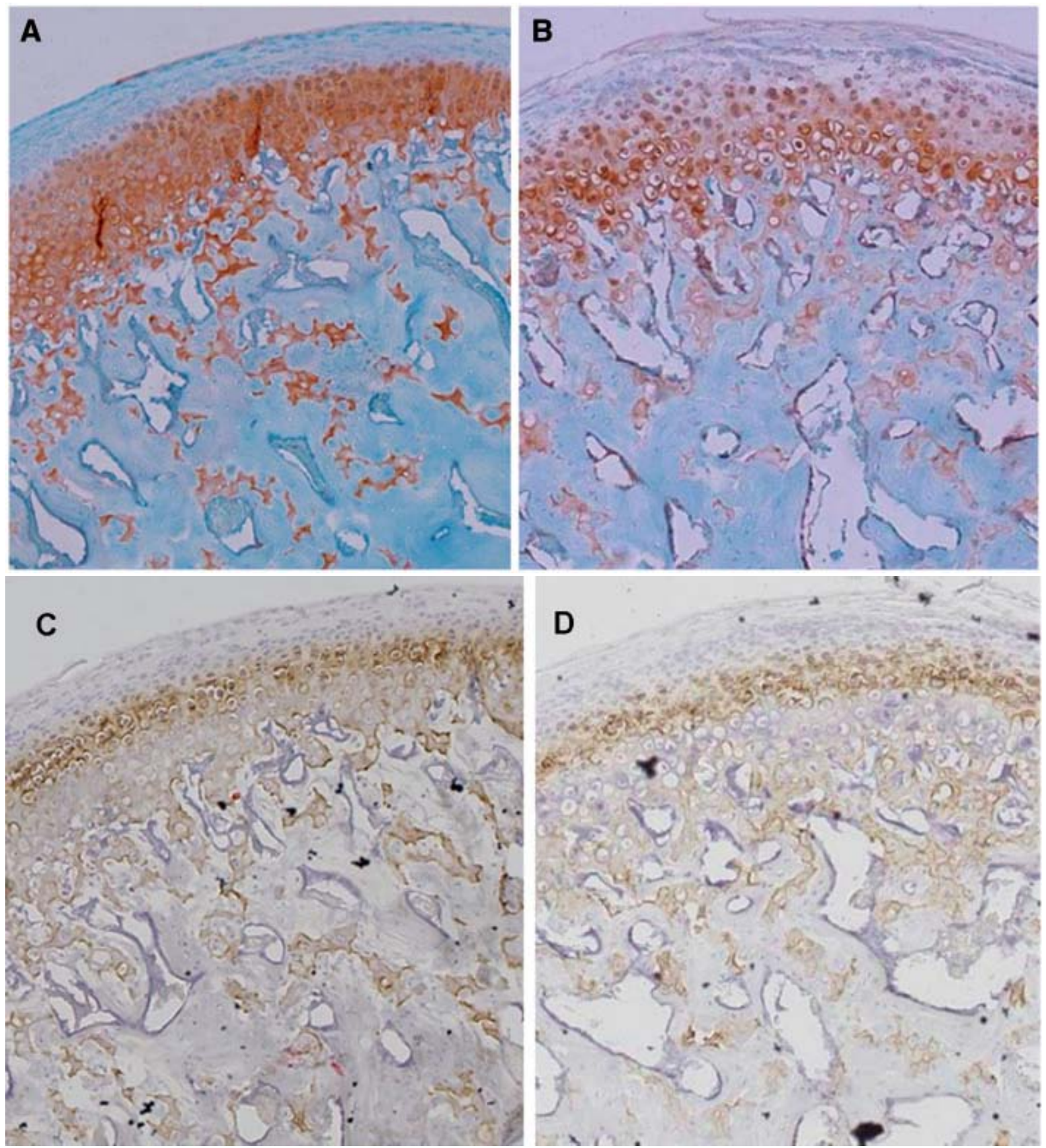

Figure 1.2 Histology and immunohistochemistry of hyaline cartilage tissue. Images are from joint U-diet (A\&C) and O-diet (B\&D). Safranin O were used to stain sulfated GAGs (A-B) and immunohistochemistry were probed for type II collagen (C-D). Figures are from Matthew J.Ravosa, Elisabeth K.Lopez, Rachel A.Menegaz et al. Using "Mighty Mouse" to understand masticatory plasticity: myostatin-deficient mice and musculoskeletal function. Integrative and Comparative Biology 2008;48:345-59. 
Figure 1.3
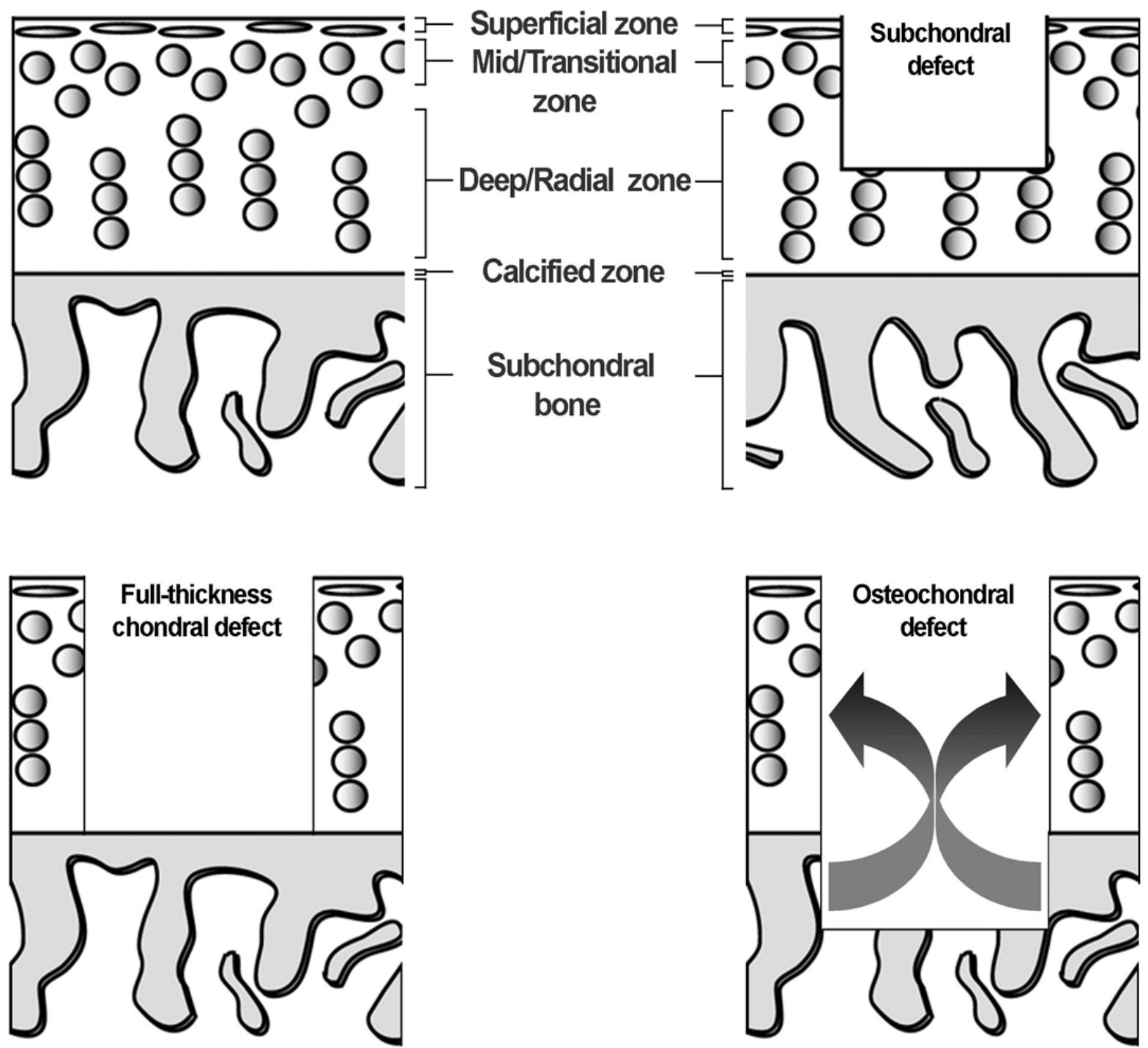

Figure 1.3 Three typical cartilage defects were met and studied in clinics, including subchondral defect, full-thickness defect and osteochondral defect. Figures are from Khan IM, Gilbert SJ, Singhrao SK et al. Cartilage integration: evaluation of the reasons for failure of integration during cartilage repair. A review. Eur.Cell Mater. 2008;16:26-39. 


\section{Figure 1.4}
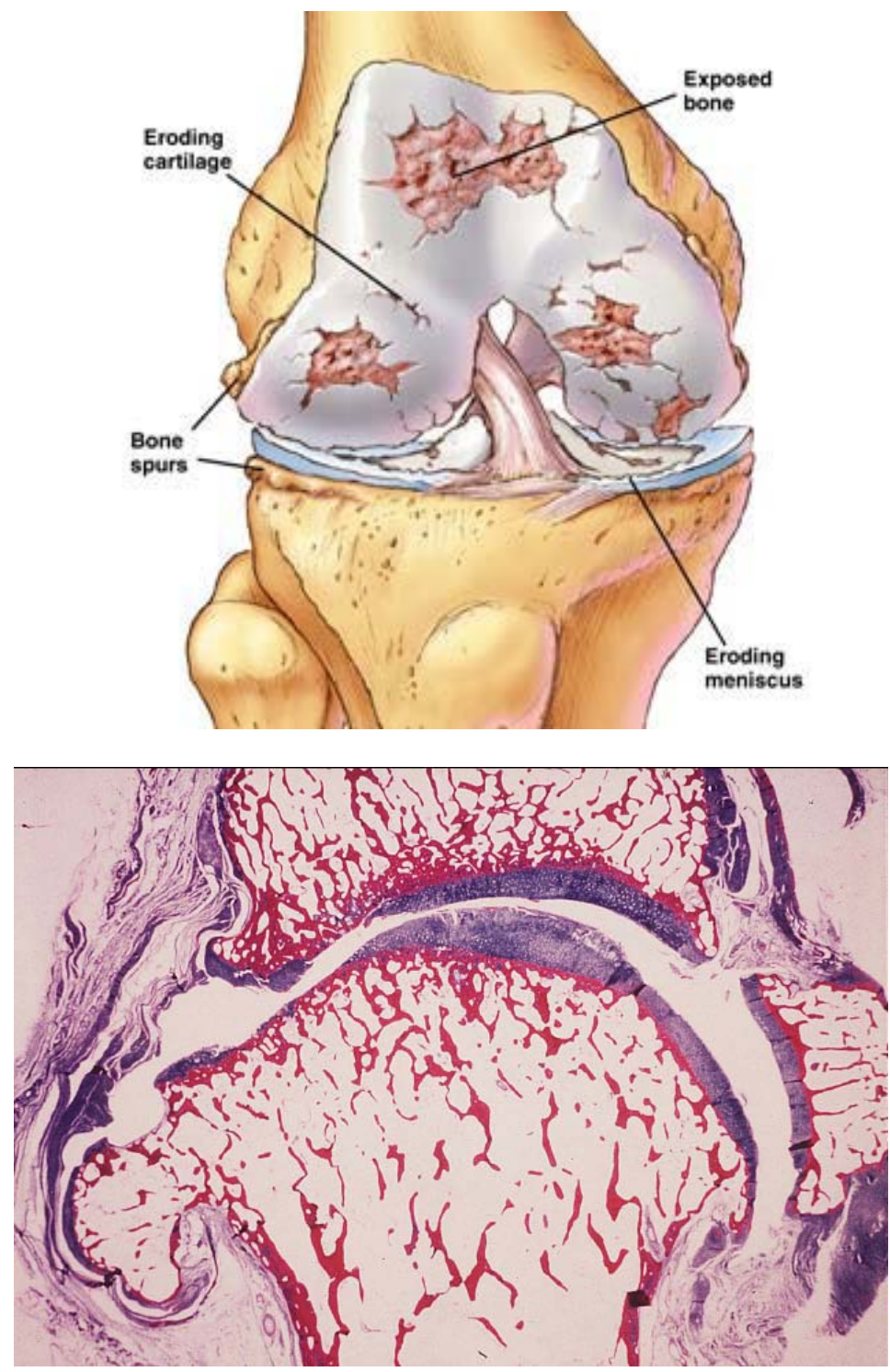

Figure 1.4 Articular cartilage is the main tissue affected by OA, which becomes softening, fibrillation, and erosion down to the subchondral bone. In the microscopic level OA results in chondrocyte cell death and the degradation of cartilage matrix. 


\section{Figure 1.5}

(A)

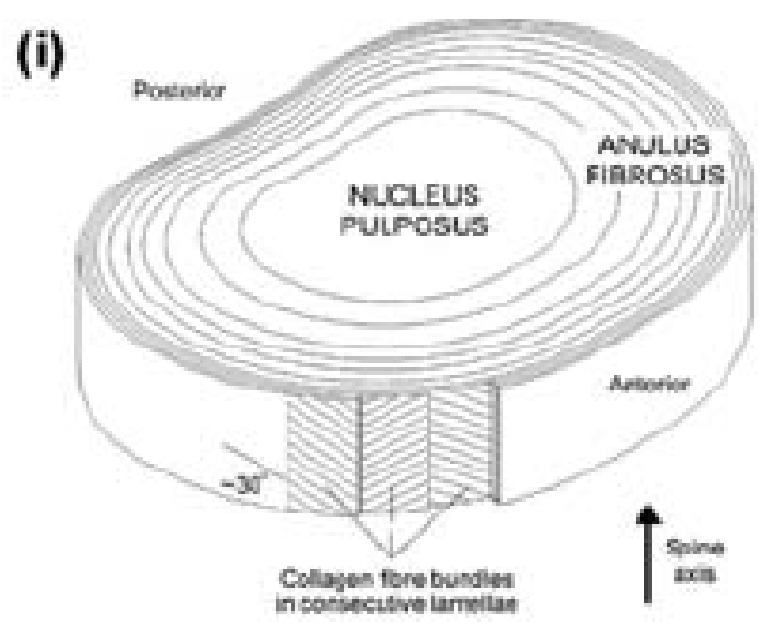

(B)

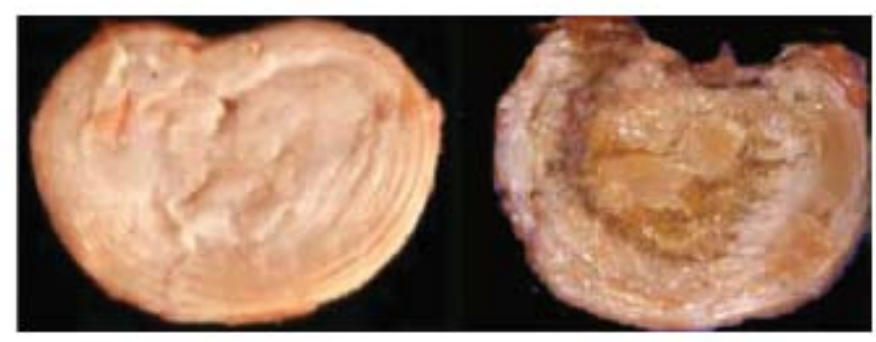

Figure $1.5(A)$ The structure of intervertebral disc contains three avascular tissues: annulus fibrosus (AF), nucleus pulposus (NP) and the cartilage endplates; (B) the degenerative disease of intervertebral disc changes the normal organized annulus and soften nucleus pulposus (left) to desiccated and disorganized tissues (right). Figure 1.5(A) from Smith LJ, Fazzalari NL. The elastic fibre network of the human lumbar anulus fibrosus: architecture, mechanical function and potential role in the progression of intervertebral disc degeneration. Eur.Spine J. 2009;18:439-48. Figure 1.5B from Urban JP, Roberts S. Degeneration of the intervertebral disc. Arthritis Res. Ther. 2003;5:120-30. 
Figure 1.6

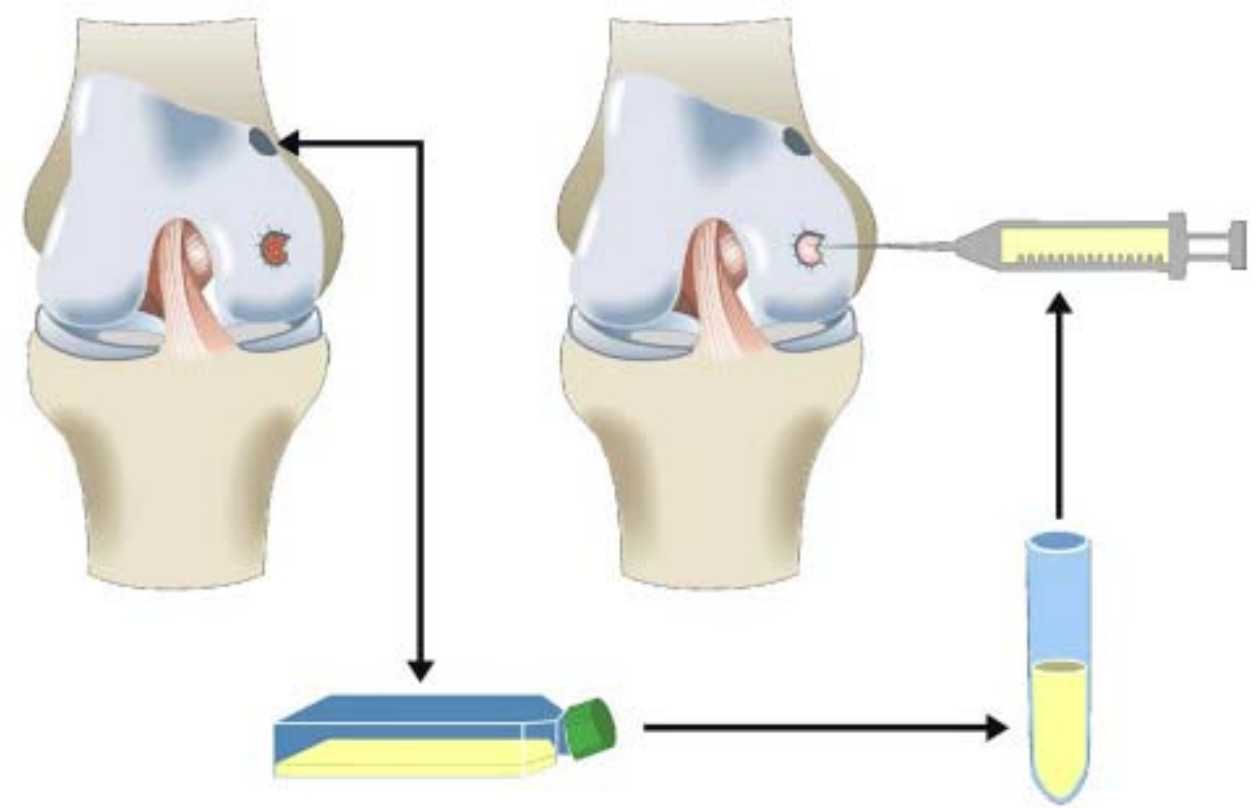

Figure 1.6 The autologous chondrocyte transplantation (ACT) technique biopsy of cartilage tissue, release of chondrocytes and expansion in vitro, and the intraarticular injection into the articular cartilage defect. Figure 1.6 from Marlovits S, Zeller P, Singer $P$ et al. Cartilage repair: generations of autologous chondrocyte transplantation. Eur.J.Radiol. 2006;57:24-31. 
Figure 1.7

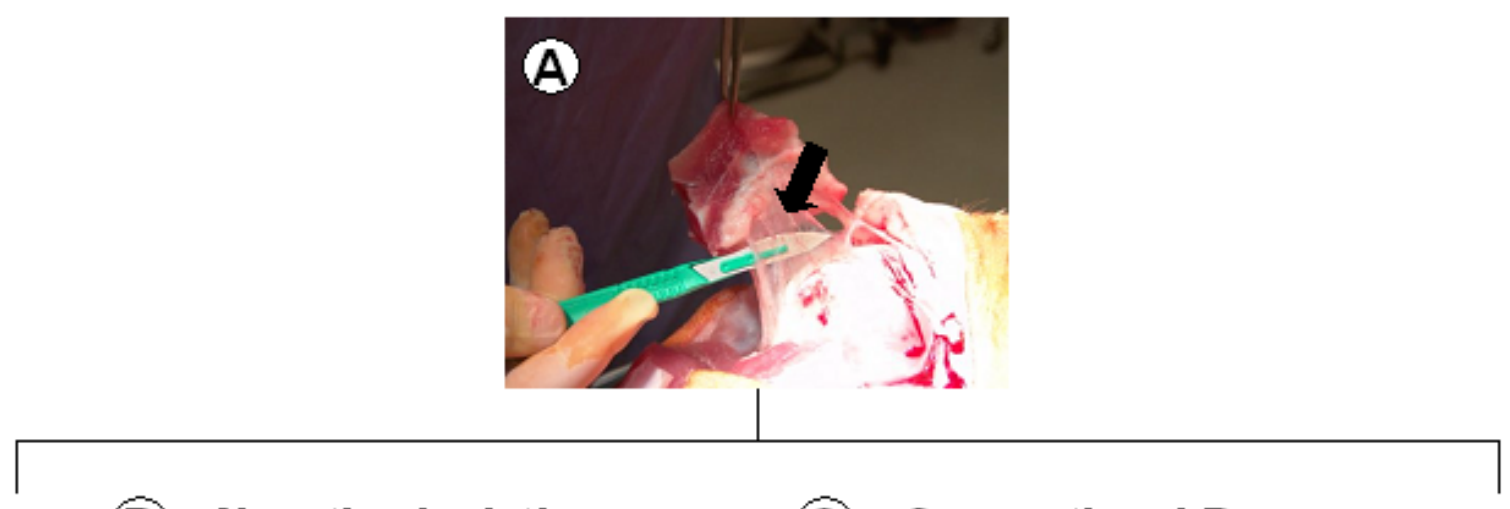

(B) Negative Isolation

(C) Conventional Passage
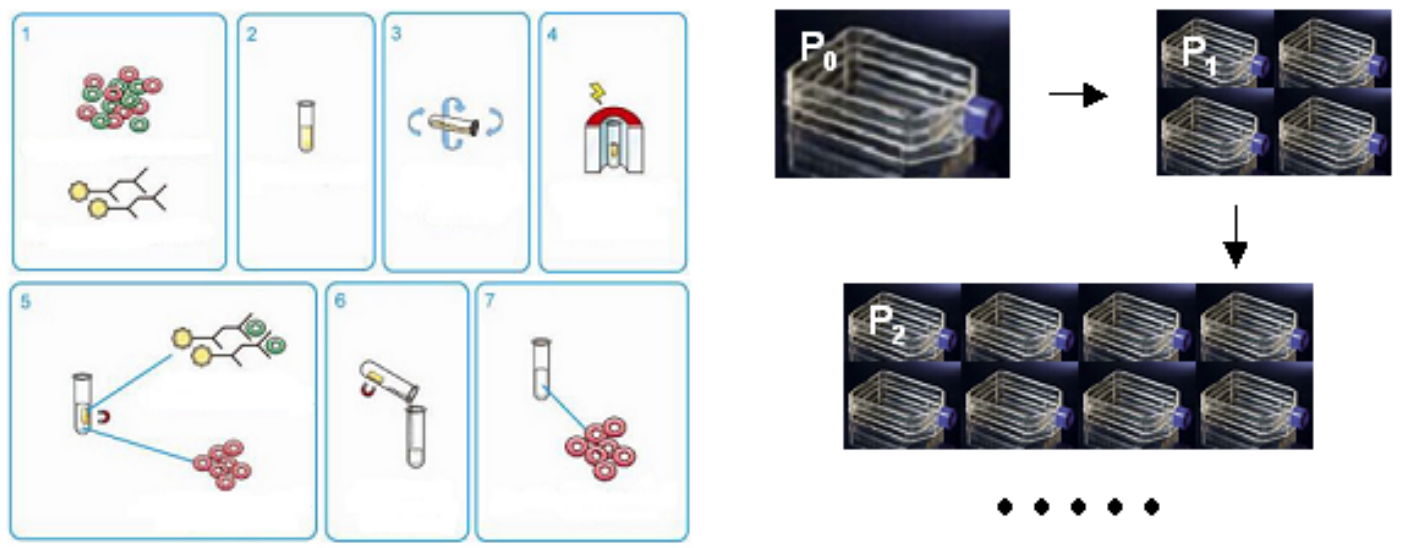

$\bullet \bullet \bullet \bullet$

Figure 1.7 The procedure of negative isolation method. Cell from the digested synovial tissue (A) are suspended and incubated with Dynabeads ${ }^{\circledR}$ M-450 CD14 conjugated with a monoclonal antibody specific for macrophages; then the conjugated cells are removed by using the Dynal Magnetic Particle Concentrator ${ }^{\circledR}$ and unconjugated cells are supposed "type B" cells (SDSCs) (B); (C) is the conventional way to isolate SDSCs by passaging. 
Figure 1.8
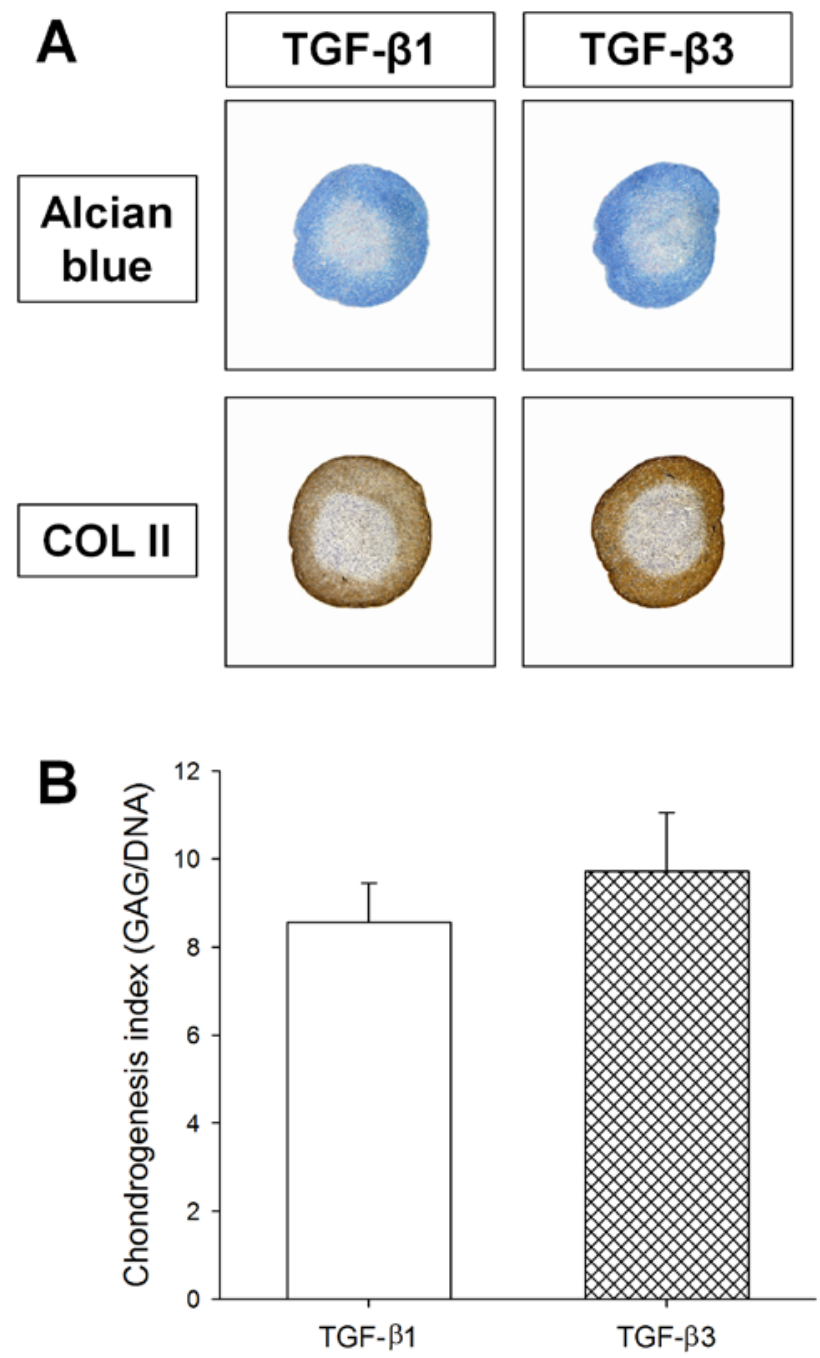

Figure 1.8 Chondrogenesis of SDSC separately induced by TGF- $\beta 1$ or TGF- $\beta 3$. (A) Alcian blue staining and immunohistochemistry of type II collagen for SDSC pellets after 14-day induction; (B) Quantification of chondrogenesis index (GAG/DNA) of SDSC pellets induced by TGF- $\beta 1$ or TGF- $\beta 3(n=5)$. 


\section{Figure 1.9}

(A)

\begin{tabular}{c|ccccc}
\hline Exp. Grp. & D1 & D2 & D3 & D4 & D5 \\
\hline Day 0-3 & $\mathrm{F}$ & $\mathrm{F}$ & $\mathrm{F}$ & $\mathrm{F}$ & $\mathrm{F}$ \\
Day 4-7 & $\mathrm{T}$ & $\mathrm{T}$ & $\mathrm{T}$ & $\mathrm{T}$ & $\mathrm{T} /$ \\
Day 8-10 & $\mathrm{T}$ & $\mathrm{T}$ & $\mathrm{T}$ & $\mathrm{T} /$ & $\mathrm{T} /$ \\
Day 11-14 & $\mathrm{T}$ & $\mathrm{T}$ & $\mathrm{T} /$ & $\mathrm{T} /$ & $\mathrm{T} /$ \\
Day 15-18 & $\mathrm{T}$ & $\mathrm{T} /$ & $\mathrm{T} /$ & $\mathrm{T} /$ & $\mathrm{T} /$ \\
\hline
\end{tabular}

(B)

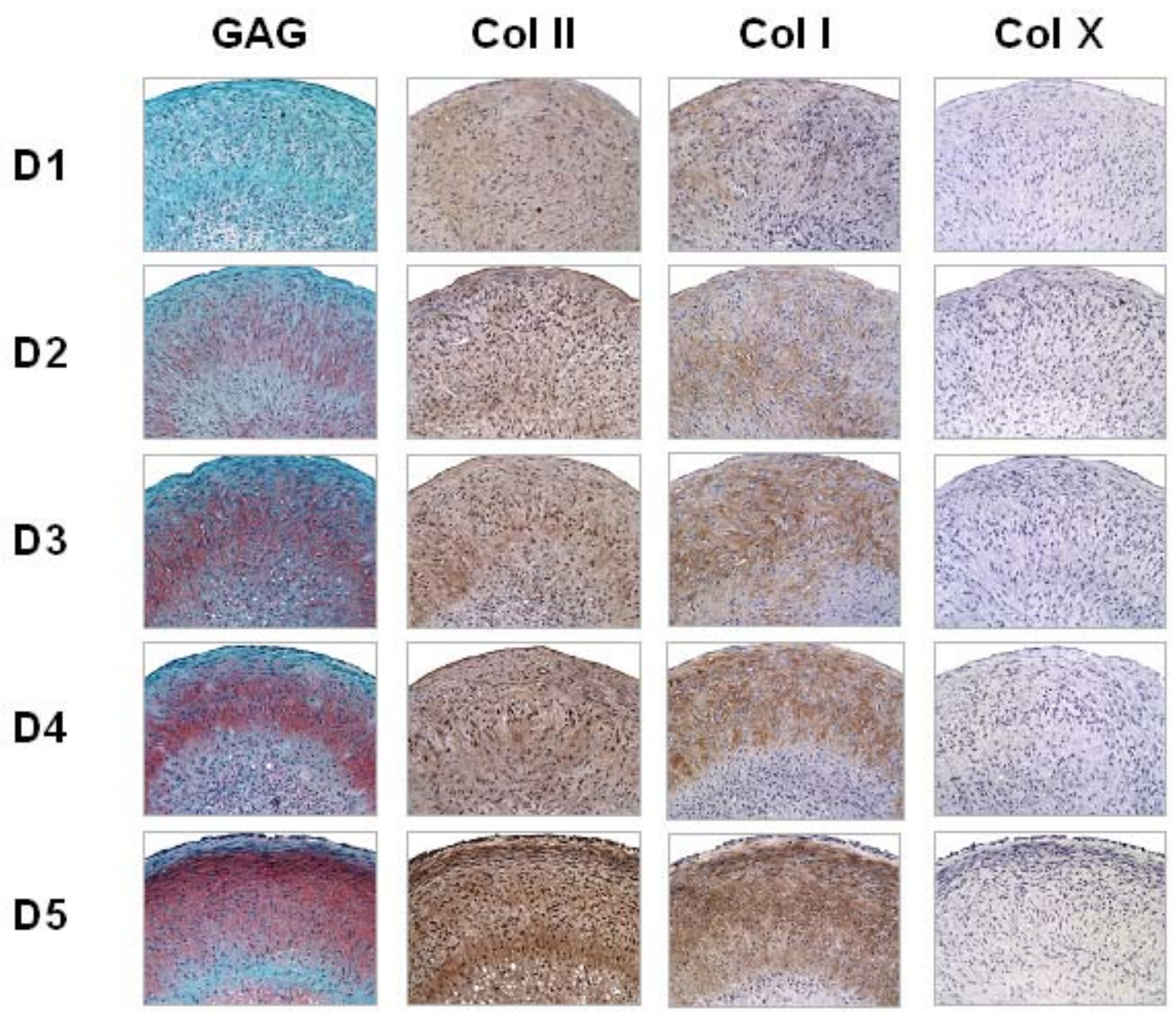

Figure 1.9 Chondrogenesis of SDSC induced by the combination of TGF- $\beta 1$, IGF-I and FGF-2. (A) the detail schedule of adoption TGF- $\beta 1$, IGF-I and FGF-2; (B) the treatment of the cocktail of TGF- $\beta 1$ and IGF-I, SDSC pellets had the strongest potential for chondrogenesis. Group D5 had the extensive expression of sulfate GAGs and type II collagen. 
Figure 1.10
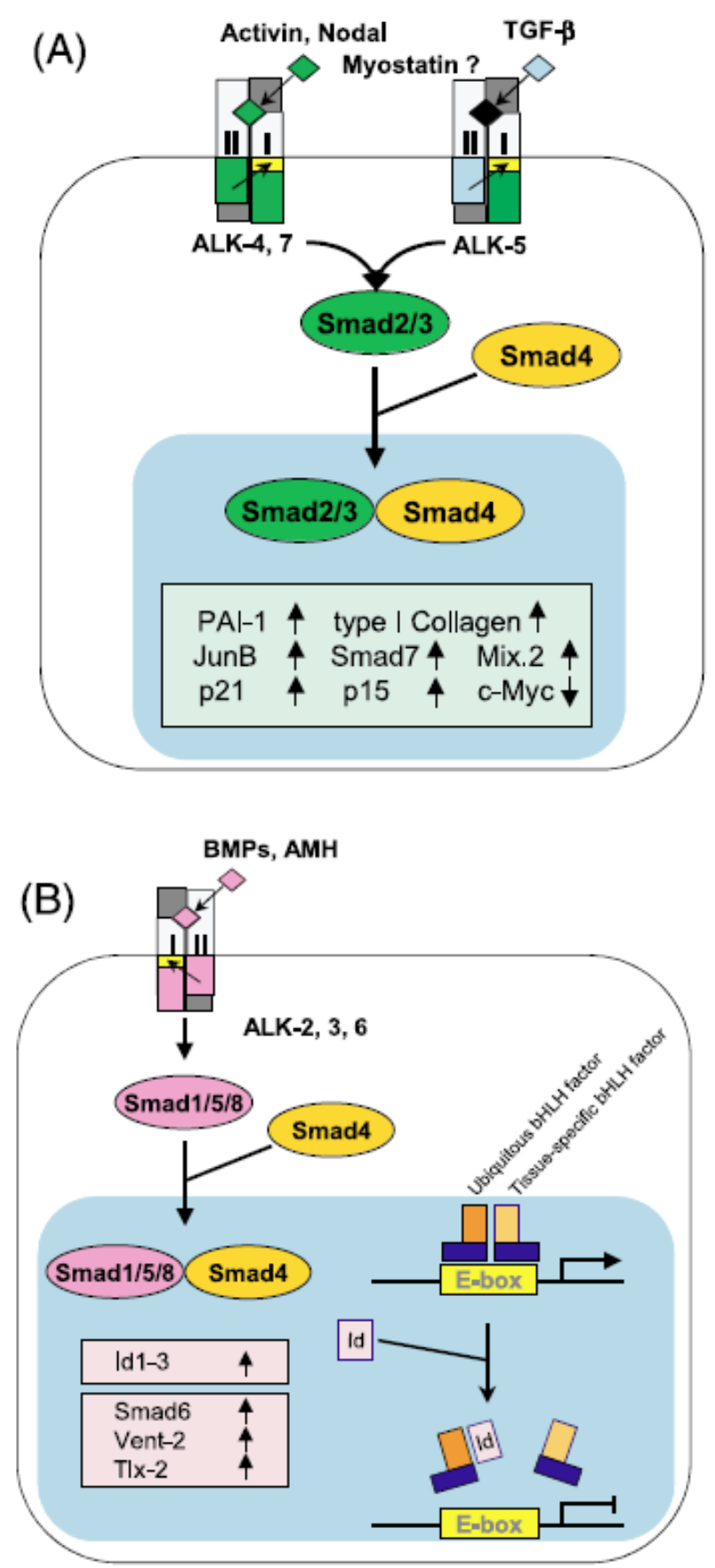

Figure 1.10 In the TGF- $\beta$ induced signaling pathway, the phosphorylated type I receptor actives R-Smads, Smad2 and Smad3 specifically. Samd2/3 associates with Co-Smad (Smad4) and the complex transfers to the nucleus to regulate the expression of target genes in cooperation with transcription factors. Figure 1.10 from Miyazawa $K$, Shinozaki M, Hara $T$ et al. Two major Smad pathways in TGF-beta superfamily signalling. Genes Cells 2002; 7:1191-204. 
Figure 1.11

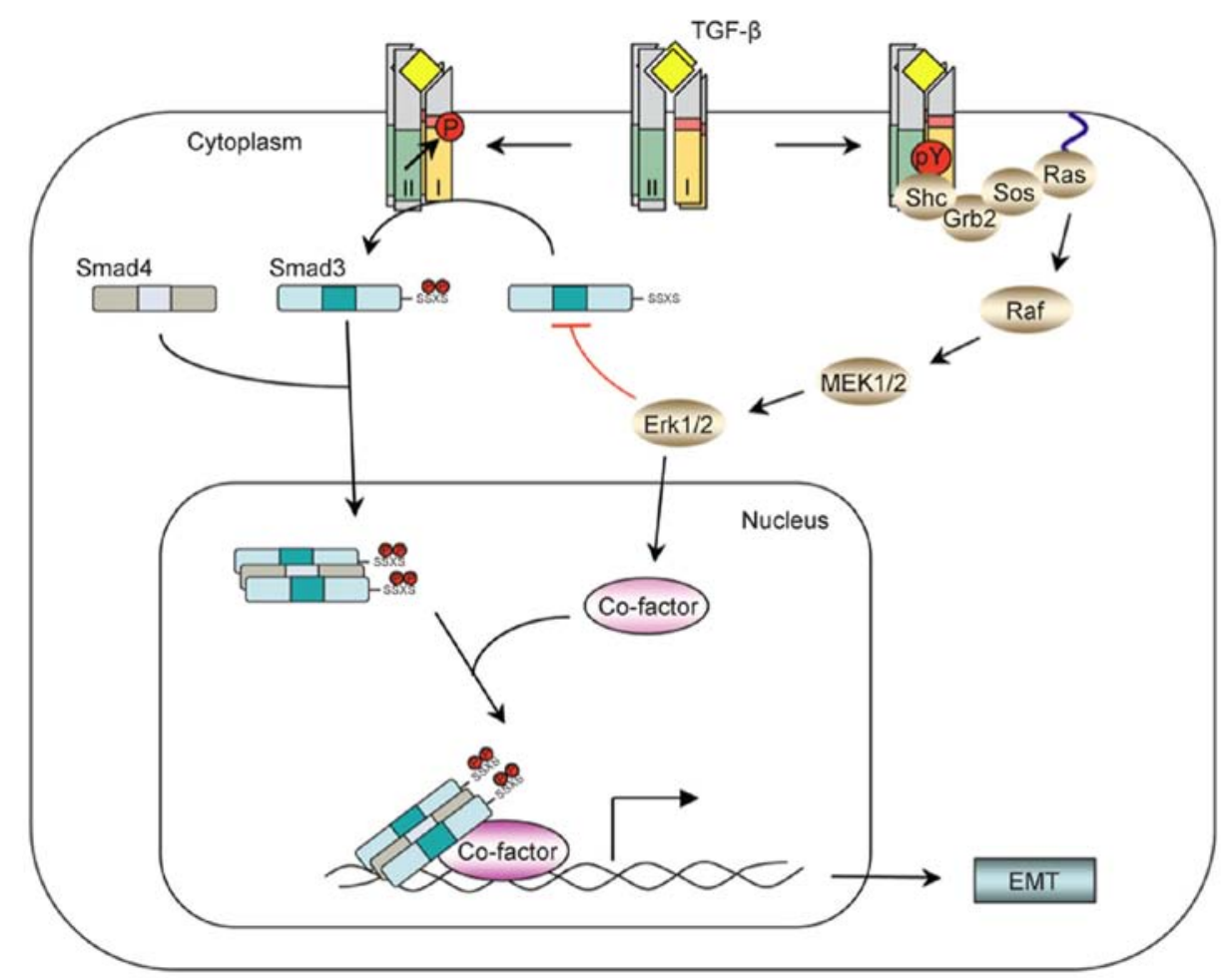

Figure 1.11 TGF- $\beta$ can induce the activation of MAPK/ERK signaling pathway. In this pathway, binding of TGF- $\beta$ to type II receptor induces the activation of type I receptor. The phosphorylation of Src or Grb2 activates Ras by catalyzing the exchange of GDP to GTP. Ras then binds to Raf and activates MAPK cascade including MEK and ERK1/2. Figure 1.11 from Zhang YE. Non-Smad pathways in TGF-beta signaling. Cell Res. 2009;19:128-39. 
Figure 1.12

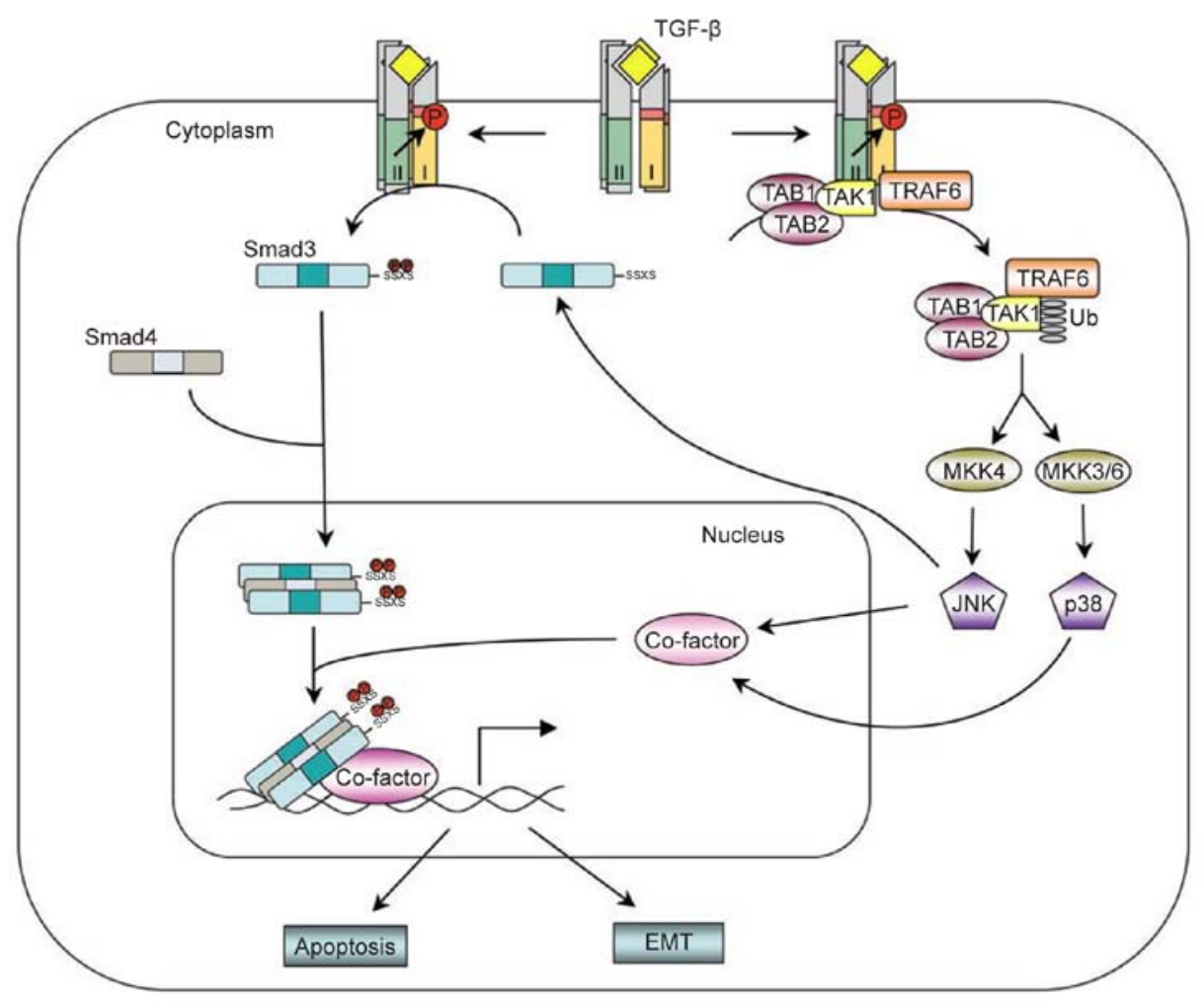

Figure 1.12 TGF- $\beta$ can induce JNK and p38 MAPK signaling cascades. TGF- $\beta$ activate JNK through MKK4 (MAP kinase kinase 4) and p38 MAPK through MKK3/6. The activated JNK and p38 MAPK move to the nucleus and initiate the expression of target genes with transcription factors Figure 1.12 from Zhang YE. Non-Smad pathways in TGF-beta signaling. Cell Res. 2009;19:128-39. 
Figure 1.13

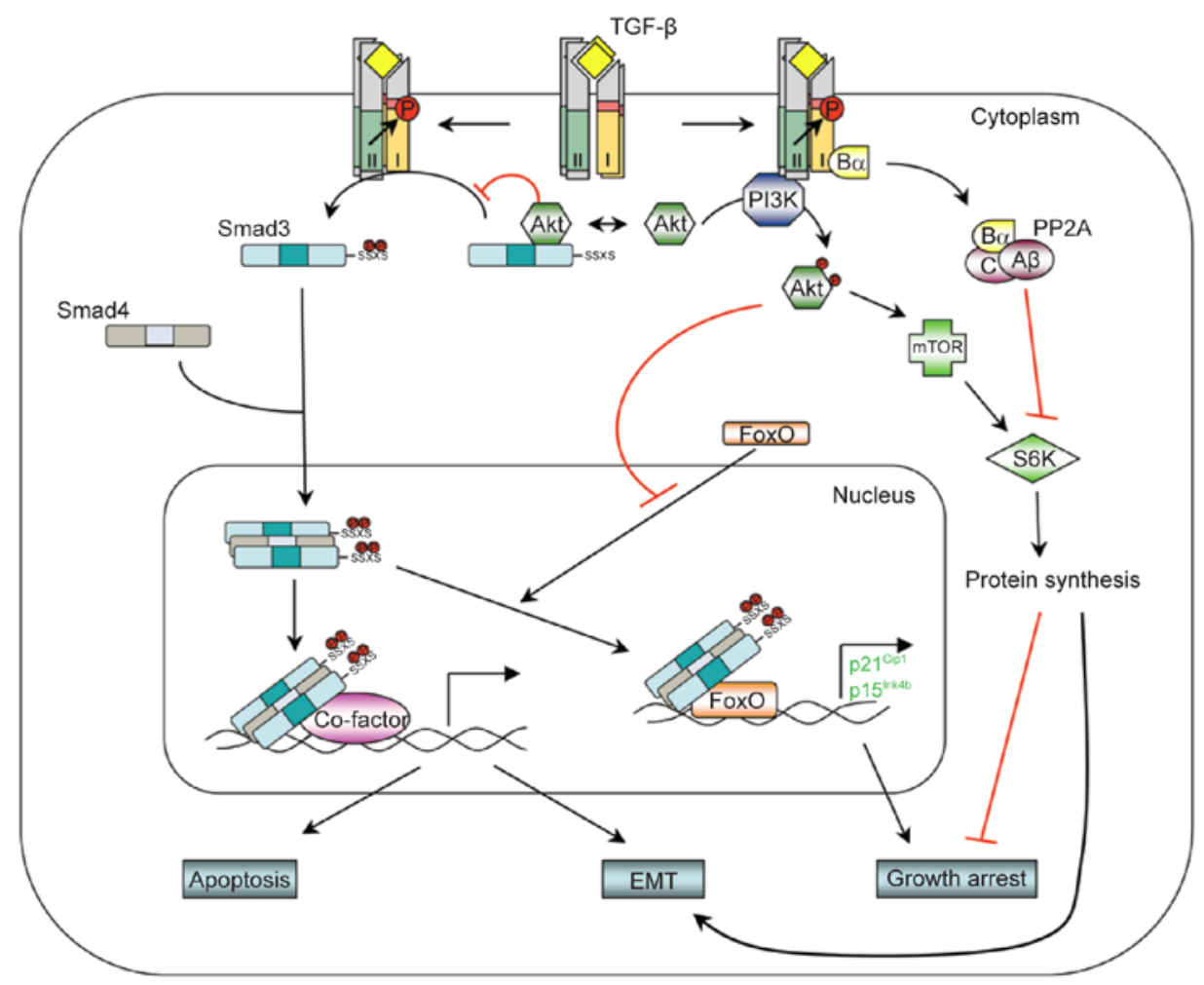

Figure 1.13 TGF- $\beta$ can induce PI3K/Akt signaling pathway. PI3K is activated by TGF- $\beta$ and then phosphorylate the downstream Akt that is independent of Smads activation. PI3K plays an important role in cell adhesion, migration and TGF- $\beta$-induced actin filament reorganization. Figure 1.13 from Zhang YE. Non-Smad pathways in TGF-beta signaling. Cell Res. 2009;19:128-39. 
Figure 1.14

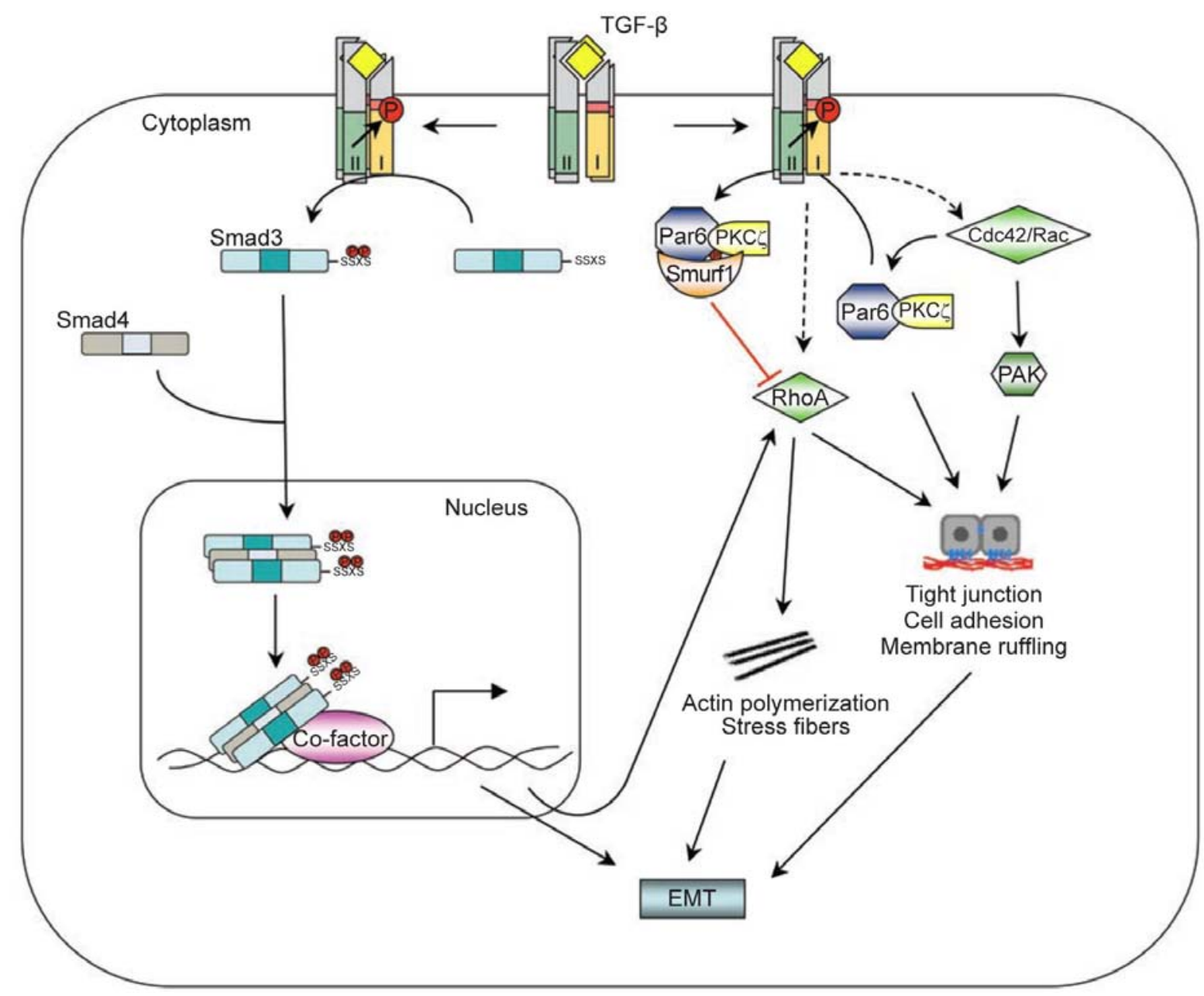

Figure 1.14 TGF- $\beta$ can activate Rho-like GTPase, including RhoA, Rac and Cdc42 to modulate the cell adhesion, proliferation and survival. The activation of RhoA and Cdc42 by TGF- $\beta$ is independent of Smads signaling pathway, indicated by the unaffected activation of p21-activated kinase (PAK) in the inhibition of Smad2/3 phosphorylation Figure 1.14 from Zhang YE. Non-Smad pathways in TGF-beta signaling. Cell Res. 2009;19:128-39. 
Figure 1.15

GAG

(day 3)

GAG

(day 15)

(1 month)

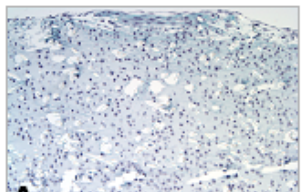

A.
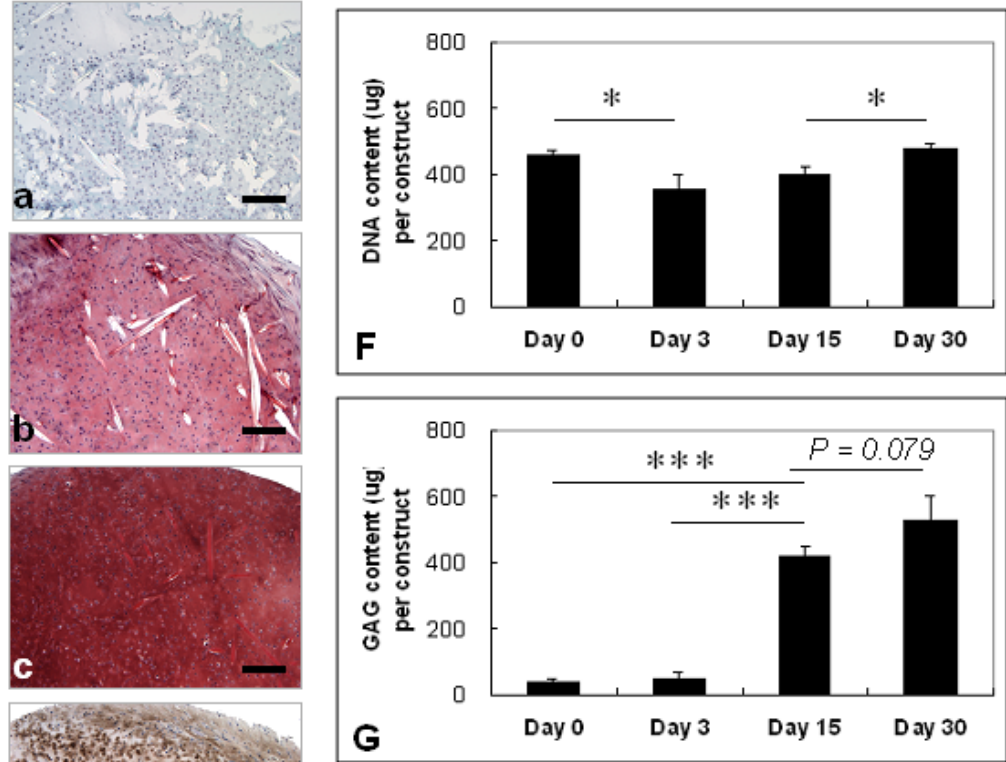

Col II

(1 month)
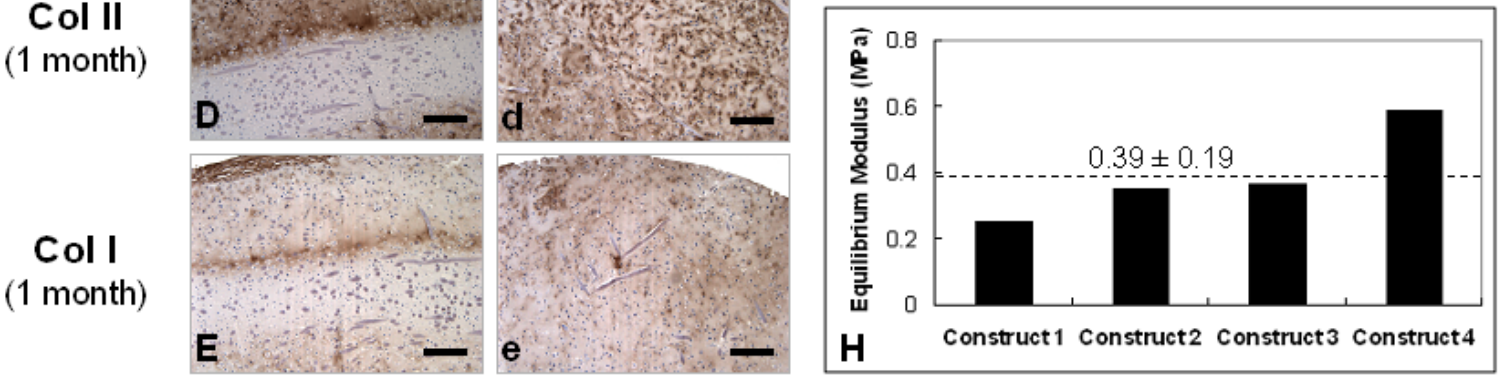

Figure 1.15 The engineered SDSC constructs were strongly positive for sulfated glycosaminoglycans and type II collagen. Hypertrophic marker, type X collagen was negative. The mechanical analysis suggested that the SDSC constructs had the similar equilibrium modulus to the nature hyaline cartilage. 


\section{Figure 1.16}
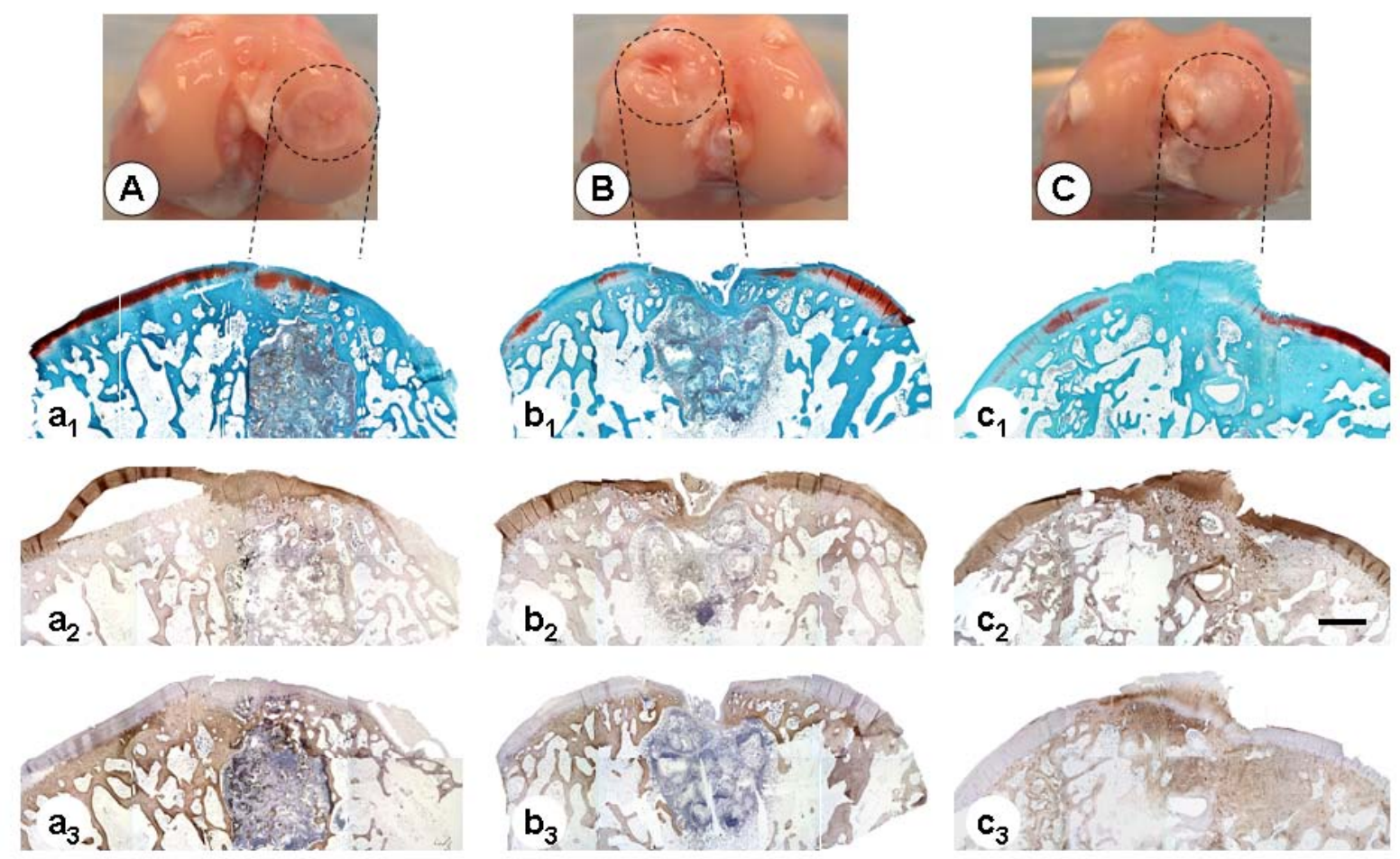

Figure 1.16 The engineered constructs using SDSCs successfully repaired the full-thickness defects in articular cartilage. Group A was repaired with SDSC constructs; Group B was repaired with only cell-free scaffolds; Group C was control group with saline. 


\section{Chapter 2:}

Reconstruction of An In Vitro Tissue-Specific Microenvironment to Rejuvenate Synovium-Derived Stem Cells for Cartilage Tissue Engineering

As published in Tissue Eng Part A 2009;15:3809-21.

Fan He, B.S. and Ming Pei, M.D., Ph.D.

Stem Cell and Tissue Engineering Laboratory, Department of Orthopaedics and Division of Exercise Physiology, West Virginia University, Morgantown, West Virginia 26506

Key words: Stem cell niche, Extracellular matrix, Cartilage regeneration, Synovium, Adult stem cell

Running head of the title: IN VITRO NICHE FOR SYNOVIUM-DERIVED STEM CELL CHONDROGENESIS 


\begin{abstract}
Joint injury results in cartilage lesions that are characterized by a poor repair response. Adult stem cells are immensely appealing for biological joint repair, such as cartilage tissue engineering and regeneration. However, adult stem cells gradually lose their stemness once they are removed from their in vivo niche for plating in plastic flasks. We utilized a tissue-specific stem cell, synovium-derived stem cell (SDSC), as a model to reconstruct an in vitro three dimensional (3D) stem cell niche. After seeding on SDSC-derived extracellular matrix (ECM), the initially wide and flat SDSCs became thin and spindle-shaped and were arranged in a 3D configuration with typical stem cell phenotypes. A dramatic increase in cell number and a greatly enhanced chondrogenic capacity were observed, though surprisingly the ECM-treated SDSCs did not display concomitantly improved adipogenic or osteogenic potentials. Thus, we conclude that a tissue-specific stem cell can be used to prepare its own in vitro niche for stem cell proliferation while maintaining and enhancing its lineage-specific stemness. The ability to reconstitute the in vitro stem cell niche will greatly benefit SDSC-based therapy for cartilage defects.
\end{abstract}




\section{INTRODUCTION}

Adult stem cells are immensely appealing for tissue engineering and therapeutic applications because of their general multipotentiality and relative ease of isolation from numerous tissues; they are currently a leading candidate for a renewable source of transplantable cells. The effectiveness of stem cell therapy is facilitated by the ability to manipulate the signals that determine stem cell behavior in a temporal and spatially appropriate fashion, both at the treatment target site as well as during in vitro processing prior to transplantation. In vitro expansion represents a formidable challenge since stem cells are thought to exist in niches where extrinsic signals modulate the intrinsic signals that drive self-renewal and cell fate determination. ${ }^{1,2}$ However, outside of their niche, adult stem cells lose their developmental potential quickly ${ }^{3}$ and tend to either randomly differentiate or undergo apoptosis over time ${ }^{4}$. Though a cocktail of growth factors can be used to increase the number of cells dramatically, the cells do not maintain stem cell characteristics. Instead, they become more mature, differentiated progenitor cells which eventually exhaust themselves after having divided a specific number of times. Therefore, one of the major challenges is controlling the fate of stem cells outside their natural environment.

In order to successfully translate human adult stem cell biology into applications in regenerative medicine, it is important to recreate a functional stem cell niche in vitro that consists entirely of known, xeno-free, and chemically defined factors and molecules. ${ }^{5}$ Studies on Drosophila indicate that when germ stem cell (GSC) niches are experimentally "emptied" of their GSCs, the remaining niches can signal incoming somatic stem cells to take up foreign residence and at least transiently maintain some of their stem cell features ${ }^{6}$ and even reverse the fate of already differentiating cells. ${ }^{7}$ Here we hypothesized that synovium-derived stem cell (SDSC)-derived extracellular matrix (ECM) can serve as an in vitro three dimensional (3D) niche, facilitating SDSC propagation and allowing restoration of stem cell stemness toward chondrogenesis. In our previous studies, we negatively isolated SDSCs from harvested synovial tissue ${ }^{8}$ for in vitro cartilage tissue engineering ${ }^{9}$ and in vivo tissue transplantation. ${ }^{10}$ SDSCs are reported as a tissue-specific stem cell for chondrogenesis. ${ }^{11-14}$ Using minimally 
invasive arthroscopic techniques, it is possible to harvest synovial tissue from patients from which SDSCs can be isolated. This brings up the intriguing possibility of autologous transplantation therapy, whereby these isolated SDSCs can be modified and retransplanted.

To date, we know of no studies that present a stem cell-derived ECM exhibiting a 'niche effect' on stem cell proliferation while maintaining stemness for a tissue-specific lineage except one study that demonstrated that ECM induced differentiation. ${ }^{15}$ Here we negatively purified SDSCs from pig synovial cells and engineered SDSC-derived 3D ECM. Partially differentiated SDSCs (Passage 3) were plated in plastic flasks either with or without ECM coating for two consecutive passages. The SDSCs grown on 3D ECM exhibited more of the properties typical of native stem cells compared with those plated on plastic flasks. ECM-treated SDSCs also displayed a remarkably enhanced chondrogenic capacity but not adipogenic or osteogenic potential. 


\section{MATERIALS AND METHODS}

Isolation and culture of SDSCS

Two 3-month-old pigs were collected from a local slaughterhouse and harvested to provide synovial tissue from both knees. The synovial tissue was finely minced and digested at $37^{\circ} \mathrm{C}$ for $30 \mathrm{~min}$ in $0.1 \%$ trypsin (Roche) and then for $2 \mathrm{~h}$ in $0.1 \%$ collagenase $\mathrm{P}$ (Roche) to release SDSCs. SDSCs were collected from the filtrate by centrifugation and plated in complete medium [ $\alpha$ MEM containing 10\% fetal bovine serum (FBS), $100 \mathrm{U} / \mathrm{mL}$ penicillin, $100 \mu \mathrm{g} / \mathrm{mL}$ streptomycin and $0.25 \mu \mathrm{g} / \mathrm{ml}$ fungizone (Invitrogen)]. Nonadherent cells were removed by medium change every two days. For negative isolation of synovial fibroblasts from primary cultures, cell suspension $\left(10^{7}\right.$ cells $\left./ \mathrm{mL}\right)$ was incubated with $5 \times 10^{7} / \mathrm{mL}$ Dynabeads ${ }^{\circledR} \mathrm{M}-450$ CD14 containing a monoclonal antibody specific for macrophages (Dynal Biotech) at $4^{\circ} \mathrm{C}$ for 1 h. The conjugated cells and the unbound Dynabeads ${ }^{\circledR}$ were collected using the Dynal Magnetic Particle Concentrator ${ }^{\circledR}$ (Dynal Biotech), and the depleted supernatant with synovial fibroblasts (referred to as $\operatorname{SDSCs}^{8}$ ) was saved for further passaging.

\section{Preparation of SDSC-derived ECM}

Fibronectin ( $1 \mu \mathrm{g} / \mathrm{cm}^{2}$, BD Bioscience) was pre-coated on plastic flasks at $37^{\circ} \mathrm{C}$ for $1 \mathrm{~h}$. Passage 2 SDSCs were plated in $175-\mathrm{cm}^{2}$ flasks at a density of $6 \times 10^{3} \mathrm{cells} / \mathrm{cm}^{2}$ in complete medium for 7 days. After SDSCs reached 90\% confluence, $50 \mu \mathrm{M}$ L-ascorbic acid phosphate (Wako) was added for an additional 8 days. To obtain cell-free ECM, ECM was incubated with 0.5\% Triton X-100 (Sigma) containing 20 mM ammonium hydroxide (Sargent-Welch) in PBS for $5 \mathrm{~min}$ at $37^{\circ} \mathrm{C}$. ECM was stored in D-PBS containing $100 \mathrm{U} / \mathrm{mL}$ penicillin, $100 \mu \mathrm{g} / \mathrm{mL}$ streptomycin and $0.25 \mu \mathrm{g} / \mathrm{mL}$ fungizone at $4^{\circ} \mathrm{C}$.

\section{SDSCS grown on SDSC-derived ECM}

Passage 3 SDSCs were plated at 3,000 cells $/ \mathrm{cm}^{2}$ in plastic flasks for two passages in two groups: ECM-coated flasks (ECM) or plain plastic flasks (Plastic). The medium was replaced every three days. The cell number from both treatments at P4 and P5 was counted using a 
counting hemocytometer for cell proliferation rate.

\section{Scanning Electron Microscope}

Representative samples $(n=2)$ were primarily fixed for $2 \mathrm{~h}$ at room temperature in $2.5 \%$ glutaraldehyde (Sigma). Secondary fixation was completed for another $2 \mathrm{~h}$ in $2 \%$ osmium tetroxide (Sigma). The samples were then dehydrated consecutively in 25\%, 50\%, 75\%, 95\%, and $100 \%$ (twice) ethanol for 10 min each, in hexamethyldisilazane (HMDS, Sigma) at a ratio of 1:1 with ethanol twice for one hour each time, in HMDS at a ratio of 1:2 with ethanol overnight, and in HMDS 3 times for $4 \mathrm{~h}$ each time. The samples were freeze-dried for $24 \mathrm{~h}$ and added with gold sputter. The images were recorded by a scanning electron microscope (Hitachi, Model S 2400).

\section{FACS analysis}

$0.5 \times 10^{6}$ SDSCs were incubated for 30 min on ice in PBS containing 5\% FBS and $1 \% \mathrm{NaN}_{3}$ (Sigma), and then for 30 min on ice with appropriately diluted mouse monoclonal antibodies to CD29 (Abcam), CD44 (Abcam), CD45 (Abcam), CD68 (BD Pharmingen), CD90 (BD Pharmingen), and CD105 (GeneTex Inc.) or isotype-matched IgGs [IgG1 and IgG2a (Beckman Coulter)]. After washing with cold PBS, SDSCs were incubated with the secondary antibody [FITC-conjugated goat anti-mouse Ig (Abcam)] for $30 \mathrm{~min}$ in the dark followed by fixation in $400 \mu \mathrm{L}$ of $1 \%$ paraformaldehyde. Negative control samples received equivalent amounts of isotype-matched pre-immune antibodies. The cells were analyzed on a BD dual laser FACSCalibur (BD Biosciences) using the FCS Express 3 software package.

\section{Chondrogenic differentiation of SDSCS}

$0.3 \times 10^{6}$ of SDSCs, either with or without ECM treatment, from P3 and P5 were placed in 15-ml polypropylene tubes and centrifuged at $500 \mathrm{~g}$ for $5 \mathrm{~min}$ to form pellets. The pellets were cultured in chondrogenic medium consisting of high-glucose DMEM, $40 \mu \mathrm{g} / \mathrm{mL}$ proline, 100 $\mathrm{nM}$ dexamethasone, $100 \mathrm{U} / \mathrm{mL}$ penicillin, $100 \mu \mathrm{g} / \mathrm{mL}$ streptomycin, $0.1 \mathrm{mM}$ ascorbic 
acid-2-phosphate and $1 \times$ ITS $^{\mathrm{TM}}$ Premix (BD Biosciences) with the supplementation of 10 ng/mL TGF- $\beta 1$ (R\&D) for 14 days.

\section{Adipogenic differentiation of SDSCS}

After $95 \%$ cell confluence, the culture medium was switched to adipogenic medium consisting of growth medium (low glucose DMEM, 10\% FBS, $100 \mathrm{U} / \mathrm{mL}$ penicillin, $100 \mu \mathrm{g} / \mathrm{mL}$ streptomycin) supplemented with $10^{-6} \mathrm{M}$ dexamethasone, $0.5 \mathrm{mM}$ isobutyl-1-methy xanthine, $100 \mu \mathrm{M}$ indomethacin and $10 \mu \mathrm{g} / \mathrm{mL}$ insulin for an additional 21 days. Adipogenic differentiation was assessed using Oil Red O stain for adipose oil and TaqMan ${ }^{\circledR}$ real time PCR for quantification expression of adipogenic marker genes.

Osteogenic differentiation of SDSCS

After $95 \%$ cell confluence, the culture medium was switched to calcification medium consisting of growth medium supplemented with $10^{-7} \mathrm{M}$ dexamethasone, $10 \mathrm{mM} \beta$-glycerol phosphate, $0.2 \mathrm{mM}$ ascorbate-2-phosphate, and L-glutamine for an additional 21 days. Osteogenic differentiation was assessed using alizarin red stain for ECM calcification. TaqMan $^{\circledR}$ real time PCR was used to semi-quantitatively evaluate osteogenic marker gene expression.

\section{Histochemistry and immunohistochemistry}

The pellets $(n=3)$ were fixed overnight at $4^{\circ} \mathrm{C}$ in $4 \%$ paraformaldehyde, followed by dehydrating in a gradient ethanol series, clearing with xylene, and embedding in paraffin blocks. 5- $\mu \mathrm{m}$ sections were histochemically stained with alcian blue (Sigma) and Safranin O (Sigma). For immunohistochemical analysis, the sections were immunolabeled with primary antibodies against collagen II (II-II6B3), collagen I (Abcam), and collagen X (Sigma), followed 
by the secondary antibody of biotinylated horse anti-mouse IgG (Vector). Immunoactivity was detected using Vectastain $A B C$ reagent (Vector) with 3,3'-diaminobenzidine (DAB) as a substrate.

\section{Biochemical analysis for DNA and GAG content}

For biochemical analysis, the pellets $(n=6)$ were digested for $4 \mathrm{~h}$ at $60^{\circ} \mathrm{C}$ with $125 \mu \mathrm{g} / \mathrm{mL}$ papain in PBE buffer (100 mmol/L phosphate, $10 \mathrm{mmol} / \mathrm{L}$ EDTA, pH 6.5) containing 10 $\mathrm{mmol} / \mathrm{L}$ cysteine, by using $100 \mu \mathrm{L}$ enzyme per sample. To quantify cell density, the amount of DNA in the papain digestion was measured using the Quanti ${ }^{\mathrm{TM}}{ }^{\mathrm{P}}$ icoGreen ${ }^{\circledR}$ dsDNA assay kit (Invitrogen) with a CytoFluor ${ }^{\circledR}$ Series 4000 (Applied Biosystems). GAG was measured using dimethylmethylene blue dye and a Spectronic ${ }^{\mathrm{TM}}$ BioMate $^{\mathrm{TM}} 3$ Spectrophotometer (Thermo Scientific) with bovine chondroitin sulfate as a standard.

\section{Western blot}

To assess non-dissolvable collagen II expression in extracellular matrix, SDSC pellets $(n=3)$ were lyophilized and incubated in $4 \mathrm{M}$ guanidine hydrochloride ( $\mathrm{GuHCl}$, Sigma) for $20 \mathrm{~h}$ at $4^{\circ} \mathrm{C}$, then digested with $0.6 \mathrm{mg} / \mathrm{ml}$ of pepsin (Sigma) in $0.5 \mathrm{M}$ acetic acid at $4^{\circ} \mathrm{C}$ for $48 \mathrm{~h}$ at a ratio of 10:1 $(\mathrm{mg} / \mathrm{mg})$ dry sample to pepsin. The samples were centrifuged at $48,000 \mathrm{~g}$ for $1 \mathrm{~h}$, and the supernatant was lyophilized and dissolved in radioimmunoprecipitation assay (RIPA) buffer with protease inhibitors (Pierce). To assess dissolvable collagen II expression in cytoplasm, the pellets were homogenized and dissolved in RIPA buffer with protease inhibitors. Total proteins were quantified using $\mathrm{BCA}^{\mathrm{TM}}$ Protein Assay Kit (Pierce). $40 \mu \mathrm{g}$ protein from each sample was denatured and separated using NuPAGE ${ }^{\circledR}$ Novex ${ }^{\circledR}$ Bis-Tris Mini Gels (Invitrogen) in the XCell SureLock ${ }^{\mathrm{TM}}$ Mini-Cell (Invitrogen) at 120 volts for $3 \mathrm{~h}$ at $4^{\circ} \mathrm{C}$. Bands were transferred onto a nitrocellulose membrane (Invitrogen) using an XCell $\mathrm{II}^{\mathrm{TM}}$ Blot module (Invitrogen) at 15 volts overnight at $4^{\circ} \mathrm{C}$. The membrane was incubated with a primary monoclonal antibody against collagen II (II-II6B3) in 1\% non-fat milk in TBST for $1 \mathrm{~h}$ at room temperature ( $\beta$-actin served as an internal control), followed by the secondary antibody of HRP-conjugated goat anti-mouse (Pierce) for $40 \mathrm{~min}$ at room temperature and exposure 
using SuperSignal West Femto Maximum Sensitivity Substrate (Pierce) and CL-XPosure Film (Pierce).

\section{$\operatorname{TaqMan}^{\circledR}$ Quantitative PCR}

The total RNA was extracted from samples $(n=4)$ using an RNase-free pestle in TRIzol ${ }^{\circledR}$ (Invitrogen). $1 \mu \mathrm{g}$ of mRNA was used for reverse transcriptase (RT) with High-Capacity cDNA Archive Kit at $37^{\circ} \mathrm{C}$ for 120 min as recommended by the manufacturer (Applied Biosystems). Chondrogenic marker genes (types I, II, X collagen, Sox 9, and aggrecan), adipogenic marker genes (LPL, PPAR $\gamma$, and C/EBP $\alpha$ ), and osteogenic marker genes (OCN, SPP1, and type I collagen) were customized by Applied Biosystems as part of the Custom Taqman ${ }^{\circledR}$ Gene Expression Assays (Table 1). Eukaryotic 18S rRNA (Assay ID HS99999901_s1 ABI) was carried out as the endogenous control gene. Real-time PCR was performed with the iCycler $\mathrm{iQ}^{\mathrm{TM}}$ Multi Color RT-PCR Detection and calculated by computer software (Perkin-Elmer). Relative transcript levels were calculated as $\mathrm{X}=2^{-\Delta \Delta \mathrm{Ct}}$, in which $\Delta \Delta \mathrm{Ct}=\Delta \mathrm{E}-\Delta \mathrm{C}$, $\Delta \mathrm{E}=\mathrm{Ct}_{\mathrm{exp}}-\mathrm{Ct}_{18 \mathrm{~s}}$, and $\Delta \mathrm{C}=\mathrm{Ct}_{\mathrm{ct} 1}-\mathrm{Ct}_{18 \mathrm{~s}}$.

\section{Statistics}

The Mann-Whitney $U$ test was used for pairwise comparison in biochemistry analysis and real-time PCR data. All the statistical analysis was performed with SPSS 13.0 statistical software (SPSS Inc., Chicago, IL). P values less than 0.05 are considered statistically significant. 


\section{RESULTS}

SDSC-derived ECM serves as an in vitro 3D stem cell niche

After using SDSCs to prepare 3D ECM substrate, Passage 3 (P3) SDSCs were plated in plastic flasks with (Figure 2.1A) or without (Figure 2.1B) ECM coating at the same initial cell density for 10 days. At day 2, SDSCs grown on ECM were thinner and more spindle-shaped though both groups exhibited similar cell distribution. After 4 days, SDSCs plated on plastic flasks proliferated slowly; in contrast, SDSCs grown on ECM increased their cell number dramatically and reached confluence at day 5 [shown by phase contrast microscope (PCM)] and were arranged in order [shown by scanning electron microscope (SEM)]. At day 9, SDSCs plated on plastic flasks became wider and flattened (shown by PCM and SEM); in contrast, ECM-treated SDSCs overlapped in 3D format (shown by PCM) with tightly organized cell distribution (shown by SEM).

To assess SDSC proliferation efficiency, P3 SDSCs were plated in plastic flasks either with (red line) or without (blue line) ECM coating for two continuing passages (Figure 2.1C). In the first passage (Passage 4, P4), ECM-treated SDSCs increased in cell number 17.5-fold compared with 6.5-fold for SDSCs plated on plastic flasks. For the last passage (Passage 5, P5), ECM-treated SDSCs increased in cell number 37.9-fold compared with 2.6-fold for SDSCs plated on plastic flasks. In total, SDSCs grown on ECM yielded a total cell number 39.2-fold more than those incubated on plastic flasks after two-passage expansion.

The SDSC-derived cell-free ECM exhibited a nanosized 3D fibrillar structure (the diameter for nanofibers was approximately $45.09 \pm 8.83 \mathrm{~nm}$ ) as revealed by SEM (Figure 2.1D). Once the ECM was detached from the flasks, ECM tension switched from "stressed" to "relaxed", which is similar to a natural scaffold. Cartilage associated structural matrix proteins were assessed in SDSC-derived ECM by safranin O staining for sulfated glycosaminoglycans (GAGs) and immunostaining for collagens I, II, and X (Figure 2.1E), suggesting that the ECM was predominantly composed of collagen I. 
SDSCs grown on SDSC-derived ECM regain stem cell phenotypes

We assessed the phenotype expression profiles from SDSCs with or without ECM treatment, including mesenchymal stem cell (MSC) surface markers (CD29, CD44, CD90, and CD105, Figure 2.2A) and a hematopoietic stem cell (HSC) marker (CD45) as well as a monocyte/macrophage marker (CD68) (Figure 2.2B). There was no CD45 or CD68 detectable in SDSCs (Figure 2.2B), probably due to monocytes and macrophages having been removed by negative selection $(8,9)$. The percentage of CD90-positive-SDSCs at P3 was $13.32 \%$ and increased dramatically to $66.15 \%$ at P4 and $79.87 \%$ at P5 when SDSCs were propagated on SDSC-derived ECM. When SDSCs were incubated on plastic flasks, however, only a small increase was observed (13.32\% at P3 versus $18.03 \%$ at P4 versus 28.15\% at P5) (Figure 2.2C). CD90 percentage data were also consistent with CD90 mean fluorescent intensity (MFI) data, which was more intense in ECM-treated SDSCs than SDSCs plated on plastic flasks (30.46 versus 15.47 at P5) (Figure 2.2D). An important indicator of chondrogenic potential ${ }^{16}$, the CD90 data suggested that ECM-treated SDSCs may regain their capacity for differentiating toward chondrogenesis. Surprisingly, the percentage of CD29-positive-SDSCs was lower in treatment with ECM than that plated on plastic flasks (81.89\% versus 96.52\%) at P5 (Figure 2.2C), which was consistent with CD29 MFI tendency (29.48 versus 57.13) (Figure 2.2D). There was no obvious difference between the two treatments in CD105-positive-SDSCs (18.78\% for ECM versus $19.71 \%$ for Plastic) and CD44-positive-SDSCs (99.31\% for ECM versus 99.19\% for Plastic) at P5 (Figure 2.2C).

\section{SDSCs propagated on ECM enhance their capacity toward chondrogenesis}

To define the potentially protective effects of SDSC-derived ECM on SDSC determination, pellets from passage 3 SDSCs grown on plastic flasks (P3.Plastic), passage 5 SDSCs grown on plastic flasks (P5.Plastic), or passage 5 SDSCs grown on ECM (P5.ECM) were incubated in chondrogenic medium for 14 days. Histochemical and immunohistochemical staining showed that SDSCs propagated on ECM displayed greatly enhanced chondrogenic potential (Figure 2.3A). At day 0 , there was no detectable positive staining in pellets from all three groups. At day 7, sulfated GAGs were intensely stained in P5.ECM pellets compared with 
much less staining in P3.Plastic pellets and were almost non-detectable in P5.Plastic pellets. At day 14, the staining trend for sulfated GAGs became more obvious with the most intense expression in P5.ECM pellets and the least expression in P5.Plastic pellets. Another key marker, collagen II, displayed a similar staining profile as sulfated GAGs. As an early marker of chondrogenesis, collagen I expression became weaker at days 7 and 14 in P5.ECM pellets in contrast with being more intense at day 14 in pellets from SDSCs plated on plastic flasks, suggesting that P5.ECM pellets undergo chondrogenic differentiation much earlier than those from either P5.Plastic or P3.Plastic. Interestingly, compared with SDSCs plated on plastic flasks, at day 14, ECM-treated SDSCs formed pellets surrounded with a unique "false membrane", which stained positively for collagen II instead of sulfated GAGs and collagen I. There was no type $X$ collagen detectable in pellets from any of the three groups.

To quantify stem cell proliferation and chondrogenic differentiation at the protein level in a pellet culture system, we performed biochemical analyses for DNA content per pellet and ratio of GAG to DNA (chondrogenic index) as well as western blot for collagen II expression in cytoplasm (immature type) and extracellular matrix (mature type). After incubation in chondrogenic medium, DNA contents (Figure 2.3C) from both SDSC pellets at P5 were shown to decrease with time. However, after day 14, DNA content in pellets from SDSCS grown on ECM was maintained until day $14(1.31 \pm 0.07 \mu \mathrm{g}$ per pellet) compared with a continuing drop in pellets from SDSCs plated on plastic flasks (1.03 $\pm 0.06 \mu \mathrm{g}$ per pellet). The chondrogenic index (Figure 2.3D) increased in pellets from all three groups with time, except for P5.Plastic pellets between day 0 and day 7. At day 14, P5.ECM SDSCs yielded pellets with the highest chondrogenic index $(39.37 \pm 3.05)$ compared with P3.Plastic SDSCs $(12.77 \pm$ 1.21), and P5.Plastic SDSCs yielded pellets that had the lowest chondrogenic index (5.19 \pm 1.11). At day 0, however, P5.Plastic SDSCs formed pellets with the highest chondrogenic index $(1.05 \pm 0.29)$ compared with P3.Plastic SDSCs $(0.47 \pm 0.11)$, while P5.ECM SDSCs formed pellets with the lowest chondrogenic index $(0.21 \pm 0.39)$, suggesting that SDSCS grown on ECM displayed not only less differentiation and highly proliferative stem cells but also tremendously enhanced chondrogenic potential. Our western blot data (Figure 2.3B) showed that collagen II protein was detectable in both cytoplasm and extracellular matrix of 
P5.ECM pellets at days 7 and 14; however, collagen II was detectable only in the cytoplasm of P5.Plastic pellets at day 14, which is consistent with our above histology and biochemical data.

We also quantitatively assessed chondrogenic marker gene expression in SDSC pellets. The mRNA profiles for collagen II (Figure 2.3E) and aggrecan (Figure 2.3F) were similar to the chondrogenic index at the protein level (Figure 2.3D). At day 7, collagen II mRNA in P5.ECM pellets was 160-fold higher than those from P3.Plastic, and 330-fold higher than those from P5.Plastic. At day 14, collagen II mRNA in P5.ECM pellets was 6-fold higher than those from P3.Plastic, and 95-fold higher than those from P5.Plastic. Sox 9 mRNA (Figure 2.3G) in P5.ECM pellets increased dramatically at day 7 and remained stable until day 14 compared with that at a low level in P5.Plastic pellets, probably accounting for the high expression of collagen II at day 7. In SDSC pellets from P5.ECM, collagen I mRNA (Figure 2.3H) transiently increased in the early phase and dropped to the lowest expression at day 14 compared with that in pellets of SDSCs plated on plastic flasks, indicating the maturity of ECM-treated SDSC pellets. In the meantime, the low level of collagen X mRNA (Figure 2.3I) suggested that ECM treatment could prevent SDSCs from hypertrophic differentiation.

\section{ECM does not retain SDSCs' potential for adipogenic and osteogenic differentiation}

Under stimulation by the cocktail for adipogenic differentiation, P3.Plastic SDSCs exhibited more lipid vacuoles than those from either P5.Plastic or P5.ECM (Figure 2.4A). Adipose-specific genes, such as lipoprotein lipase (LPL, Figure 2.4B), peroxisome proliferator-activated receptor gamma (PPARY, Figure 2.4C), and CCAAT/enhancer binding protein alpha (C/EBPa, Figure 2.4D) were quantified to assess their relative mRNA level. P3.Plastic SDSCs had the highest expression in all three adipogenic marker genes. P5.ECM SDSCs displayed higher (LPL mRNA and C/EBPa mRNA) or similar (PPARy mRNA) expression of adipogenic marker genes compared with P5.Plastic SDSCs. Our quantitative PCR data indicated that adipogenic capacity was not improved with each passage even with ECM treatment. 
Under stimulation by the cocktail for osteogenic differentiation, SDSCs underwent a change in morphology from spindle-shaped to cuboidal, and formed large nodules, which were observed with a PCM and were positive for Alizarin Red staining compared with negative controls (Figure 2.5A). This process was demonstrated by a significant increase in the expression of osteogenesis specific genes, including osteocalcin (OCN, Figure 2.5B), secreted phosphoprotein 1 (SPP1/osteopontin, Figure 2.5C) and collagen $\alpha 1$ (Col I, Figure 2.5D). In all three osteogenic marker genes, P3.Plastic SDSCs had the highest expression while SDSCs from P5.ECM SDSCs had the lowest expression, indicating SDSCs grown on ECM exhibited decreased osteogenic capacity. 


\section{DISCUSSION}

As a tissue-specific stem cell, SDSCs are promising MSCs for cartilage tissue engineering and regeneration. However, like other MSCs, SDSCs exhibit diminished proliferation and differentiation capacity when they are expanded on standard plastic flasks, which presents a number of problems in the clinical setting. In this study, we successfully engineered an in vitro 3D stem cell niche using SDSC-derived ECM for improving SDSC proliferation while enhancing SDSC stemness toward chondrogenesis. In contrast, SDSCs grown on plastic flasks displayed diminished replicative capacity and increased random multi-differentiation properties. We also demonstrated that ECM-treated SDSCs do not regain their adipogenic and osteogenic potential, suggesting that an in vitro 3D stem cell niche facilitates SDSC propagation while allowing SDSCs to retain or even reinforce stemness for chondrogenic differentiation.

The stem cell niche is a specialized microenvironment that helps sustain the stem cell pool within each tissue or organ system ${ }^{4}$. Typically, even with considerable variation in niche design, there are three key components in the niche concept: supporting niche cells, supporting ECM with growth-modulating factors, and the physical characteristics of the surrounding 3D structures. ${ }^{17,18}$ Of them, niche cells are normally a heterogeneous cell population and act to limit or control stem cell division. For instance, osteoblasts, the HSC niche cells, maintain HSC quiescence ${ }^{19}$ by producing osteopontin, which suppresses stem cell expansion ${ }^{20}$. Thus, in native tissue, a loss of niche cells may result in overproliferation of stem cells. Recently, growing evidence suggests that some stem cells can create their own

microenvironments. ${ }^{21-23}$ Stem cells may play an important role in organizing and specifying their niche, and conversely the architectural design of a niche appears to be specifically tailored to suit the particular needs of its resident stem cells. ${ }^{24}$ Tissue-specific matrices clearly influence developmental lineage choices, and thus whole tissue and organ formation. ${ }^{25}$ In such a microenvironment, adult stem cells are expected to expand greatly while retaining their stemness for a tissue-specific lineage. In our study, SDSCs were used to create their own microenvironments without supporting niche cells. When partially differentiated SDSCs were 
seeded in such a niche, the morphological transformation accelerated from wider, flattened cells to thinner, spindle-shaped cells, demonstrating that SDSC-derived ECM can restore SDSCs back to the original size and shape observed in their natural niche. After the initial lag, the cell numbers were dramatically expanded with cells growing in a 3D format. Phenotypic characterization demonstrated that SDSCs grown on ECM retained and possibly enhanced stem cell stemness. Differentiation data clearly indicated that SDSCs grown on SDSC-derived ECM regained stemness specifically toward the chondrogenic lineage but not toward adipogenic or osteogenic lineages, supporting reports that stem cell niches act to protect the self-renewing, undifferentiated state of the cells within and regulate the rate of production of committed, tissue-specific progenitors. ${ }^{26}$ Although the presence of niche cells in an in vivo niche act to limit or inhibit stem cell proliferation and maintain its multi-differentiation potential, our findings indicate that in an in vitro niche, the absence of niche cells may be associated with restoring stem cell proliferation and enhancing tissue-specific lineage differentiation capacity. The underlying mechanisms need further elucidation.

The geometry, composition, and stiffness of the ECM surrounding cells residing in their niche also have major effects on cell signaling and behavior. ${ }^{27-29}$ While ECM is known to serve as a reservoir for growth factors and cytokines, ${ }^{25}$ our study demonstrated that SDSC-derived ECM is a 3D matrix consisting of nanostructured fibers with collagen I as one of the major structural proteins. ECM adhering on plastic flasks through fibronectin exhibited substrate tension, reported to up-regulate type I collagen polymerization. ${ }^{30}$ The presence of greater amounts of type I collagen may contribute to a physical architecture that transmits different mechanical cues to their resident cells. ${ }^{31}$ Because there is a growing body of evidence that cell attachment, proliferation, and migration will be enhanced when cells grow in a more physiologically similar environment as opposed to standard 2D tissue culture substrates, ${ }^{27,31,32}$ creating such an in vitro functional niche becomes a vital step to restoring the full potential of stem cells for therapeutic applications and may also have important implications for research regarding niche-related signal transduction. For example, signaling by morphogens, particularly through the Wnt, Notch and Hedgehog pathways, is known to be important for the self-renewal of many adult stem cell types. ${ }^{33-35}$ With further study in the context of stem 
cell-derived ECM, functional in vitro niches, may implicate these signals in even more complex roles. Although most proliferative signals seem to concurrently stimulate the commitment to differentiation, ${ }^{36}$ our study suggests that an in vitro stem cell niche can encourage stem cell self-renewal and prevent both undesired differentiations and exhaustion, consequently improving the potential of the cell population as a whole. Communication within the niche is essential for the maintenance of proper stem cell function and for determining the rate of stem cell self-renewal. Further studies are needed to determine which of these ECM molecules are crucial for the maintenance of the undifferentiated stem cell phenotype, the underlying tissue-specific stem cell signalling pathways, and the mechanical interactions between the ECM and seeded cells. This in vitro stem cell niche provides a promising and reliable approach in re-programming stem cells for either investigational or therapeutic purposes. 


\section{ACKNOWLEDGEMENTS}

We would like to thank Dr. Kathleen Brundage for her kind help in performing flow cytometry and data analysis, Yunbing Tan for sample preparation for the scanning electron microscope, and Song Chen for histology sample preparation. We also would like to thank Suzanne Smith and Mark Shoukry for editing the manuscript. This study was supported by the peer-reviewed research grant from the Musculoskeletal Transplant Foundation (Project no. 10012220) and AO research fund of the AO Foundation (Project no. S-08-67P) to M.P.. 


\section{REFERENCES}

1. Song, X., Zhu, C.H., Doan, C. and Xie, T. Germline stem cells anchored by adherens junctions in the Drosophila ovary niches. Science 296, 1855, 2002.

2. Watt, F.M. and Hogan, B.L. Out of Eden: stem cells and their niches. Science 287, 1427, 2000.

3. Lin, H. The stem-cell niche theory: lessons from flies. Nat Rev Genet 3, 931, 2002.

4. Scadden, D.T. The stem-cell niche as an entity of action. Nature 441, 1075, 2006.

5. Martin, M.J., Muotri, A., Gage, F. and Varki, A. Human embryonic stem cells express an immunogenic nonhuman sialic acid. Nat Med 11, 228, 2005.

6. Kai, T. and Spradling, A. An empty Drosophila stem cell niche reactivates the proliferation of ectopic cells. Proc Natl Acad Sci USA 100, 4633, 2003.

7. Kai, T. and Spradling, A. Differentiating germ cells can revert into functional stem cells in Drosophila melanogaster ovaries. Nature 428, 564, 2004.

8. Pei, M., He, F. and Vunjak-Novakovic, G. Synovium-derived stem cell-based chondrogenesis. Differentiation 76, 1044, 2008.

9. Pei, M., He, F., Kish, V.L., Vunjak-Novakovic, G. Engineering of functional cartilage tissue using stem cells from synovial lining: a preliminary study. Clin Orthop Relat Res 466, 1880, 2008.

10. Pei, M., He, F., Boyce, B.M. and Kish, V.L. Repair of full-thickness femoral condyle cartilage defects using allogeneic synovial cell-engineered tissue constructs. Osteoarthritis Cartilage doi: 10.1016/j.joca.2008.11.017, 2008.

11. Dickhut, A., Pelttari, K., Janicki, P., Wagner, W., Eckstein, V., Egermann, M. and Richter, W. Calcification or dedifferentiation: Requirement to lock mesenchymal stem cells in a desired differentiation stage. J Cell Physiol 219, 219, 2009.

12. Mochizuki, T., Muneta, T., Sakaguchi, Y., Nimura, A., Yokoyama, A., Koga, H. and Sekiya, I. Higher chondrogenic potential of fibrous synovium- and adipose synovium-derived cells compared with subcutaneous fat-derived cells: distinguishing properties of mesenchymal stem cells in humans. Arthritis Rheum 54, 843, 2006. 
13. Sakaguchi, Y., Sekiya, I., Yagishita, K. and Muneta, T. Comparison of human stem cells derived from various mesenchymal tissues: superiority of synovium as a cell source. Arthritis Rheum 52, 2521, 2005.

14. Segawa, Y., Muneta, T., Makino, H., Nimura, A., Mochizuki, T., Ju, Y.J., Ezura, Y., Umezawa, A. and Sekiya, I. Mesenchymal stem cells derived from synovium, meniscus, anterior cruciate ligament, and articular chondrocytes share similar gene expression profiles. J Orthop Res 27, 435, 2008.

15. Datta, N., Holtorf, H.L., Sikavitsas, V.I., Jansen, J.A. and Mikos, A.G. Effect of bone extracellular matrix synthesized in vitro on the osteoblastic differentiation of marrow stromal cells. Biomaterials 26, 971, 2005.

16. Nagase, T., Muneta, T., Ju, Y.J., Hara, K., Morito, T., Koga, H., Nimura, A., Mochizuki, T. and Sekiya, I. Analysis of the chondrogenic potential of human synovial stem cells according to harvest site and culture parameters in knees with medial compartment osteoarthritis. Arthritis Rheum 58, 1389, 2008.

17. Lutton, C. and Goss, B. Caring about microenvironments. Nat Biotechnol 26, 613, 2008.

18. Moore, K.A. and Lemischka, I.R. Stem cells and their niches. Science 311, 1880, 2006.

19. Calvi, L.M., Adams, G.B., Weibrecht, K.W., Weber, J.M., Olson, D.P., Knight, M.C., Martin, R.P., Schipani, E., Divieti, P., Bringhurst, F.R., Milner, L.A., Kronenberg, H.M. and Scadden, D.T. Osteoblastic cells regulate the haematopoietic stem cell niche. Nature 425, 841, 2003.

20. Stier, S., Ko, Y., Forkert, R., Lutz, C., Neuhaus, T., Grünewald, E., Cheng, T., Dombkowski, D., Calvi, L.M., Rittling, S.R. and Scadden D.T. Osteopontin is a hematopoietic stem cell niche component that negatively regulates stem cell pool size. J Exp Med 201, 1781, 2005.

21. Bendall, S.C., Stewart, M.H., Menendez, P., George, D., Vijayaragavan, K., Werbowetski-Ogilvie, T., Ramos-Mejia, V., Rouleau, A., Yang, J., Bossé, M., Lajoie, G. and Bhatia, M. IGF and FGF cooperatively establish the regulatory stem cell niche of pluripotent human cells in vitro. Nature 448, 1015, 2007. 
22. Blanpain, C., Lowry, W.E., Geoghegan, A., Polak, L. and Fuchs, E. Self-renewal, multipotency, and the existence of two cell populations within an epithelial stem cell niche. Cell 118, 635, 2004.

23. Wagers, A. and Conboy, I.M. Cellular and molecular signatures of muscle regeneration: current concepts and controversies in adult myogenesis. Cell 122, 659, 2005.

24. Fuchs, E., Tumbar, T. and Guasch, G. Socializing with the neighbors: stem cells and their niche. Cell 116, 769, 2004.

25. Badylak, S.F. The extracellular matrix as a scaffold for tissue reconstruction. Semin Cell Dev Biol 13, 377, 2002.

26. Nurcombe, V. and Cool, S.M. Heparan sulfate control of proliferation and differentiation in the stem cell niche. Crit Rev Eukaryot Gene Expr 17, 159, 2007.

27. Cukierman, E., Pankov, R. and Yamada, K.M. Cell interactions with three dimensional matrices. Curr Opin Cell Biol 14, 633, 2002.

28. Discher, D.E., Janmey, P. and Wang, Y.L. Tissue cells feel and respond to the stiffness of their substrate. Science 310, 1139, 2005.

29. Grinnell, F. Fibroblast biology in three-dimensional collagen matrices. Trends Cell Biol 13, 264, 2003.

30. He, Y.L., Macarak, E.J., Korostoff, J.M. and Howard, P.S. Compression and tension: differential effects on matrix accumulation by periodontal ligament fibroblasts in vitro. Connect Tissue Res 45, 28, 2004.

31. Cukierman, E., Pankov, R., Stevens, D.R. and Yamada, K.M. Taking cell-matrix adhesions to the third dimension. Science 294, 1708, 2001.

32. Griffith, L.G. and Swartz, M.A. Capturing complex 3D tissue physiology in vitro. Nat Rev Mol Cell Biol 7, 211, 2006.

33. Chiba, S. Notch signaling in stem cell systems. Stem Cells 24, 2437, 2006.

34. Clevers, H. Wnt/ß-catenin signaling in development and disease. Cell 127, 469, 2006.

35. Lai, K., Kaspar, B.K., Gage, F.H. and Schaffer, D.V. Sonic hedgehog regulates adult neural progenitor proliferation in vitro and in vivo. Nature Neurosci 6, 21, 2003. 
36. Orford, K.W. and Scadden, D.T. Deconstructing stem cell self-renewal: genetic insights into cell-cycle regulation. Nat Rev Genet 9, 115, 2008. 


\section{FIGURE LEGENDS}

Figure 2.1. Effect of SDSC-derived ECM on SDSC morphology and proliferation. (A) Passage 4 SDSCs grown on SDSC-derived ECM at days 2, 5, and 9. (B) Passage 4 SDSCs grown on plastic flasks at days 2, 5, and 9. Phase contrast microscope (PCM) and scanning electron microscope (SEM) were used to evaluate SDSC morphology properties. (C) Cell proliferation from Passage 3 SDSCs grown in plastic flasks either with or without ECM coating for two consecutive passages. (D) SDSC-derived ECM attaching on plastic flasks ("stressed" ECM) or detaching from plastic flasks ("relaxed" ECM). (E) SDSC-derived ECM was stained with Safranin O for sulfated GAGs, and immunostained for chondrogenic matrix (collagens I, II, and X). All scale bars are $200 \mu \mathrm{m}$.

Figure 2.2. Quantitative flow cytometry analysis of typical cell surface antigens (red color) from MSC (CD29, CD44, CD90, CD105) (A) and HSC (CD45) as well as monocyte/macrophage (CD68) (B) with isotype-specific control. The percentage of positive cells (C) and mean fluorescent intensity (MFI) (D) were also shown for MSC phenotype changes in SDSCs grown in plastic flasks with or without ECM coating for two consecutive passages.

Figure 2.3. Distinctively chondrogenic capacity from SDSCs grown on SDSC-derived ECM. The pellets from P3 and P5 SDSCs were incubated in chondrogenic medium for 14 days. (A) Alcian blue and Safranin O staining were used to detect sulfated GAGs, and immunostaining was used to detect collagens I, II, and X. The scale bar is $800 \mu \mathrm{m}$. (B) Western blot was used to detect collagen II protein either in the pellet matrix (mature type) or in cytoplasm (immature type) of differentiated SDSCs. Three independent experiments were performed. Biochemical analyses were used to detect DNA content per pellet (C) and ratio of GAG to DNA (D). $\operatorname{TaqMan}^{\circledR}$ real time PCR was used to quantitatively assess chondrogenic markers [collagen II $(E)$, aggrecan $(F)$, Sox $9(G)$, collagen I $(H)$, and collagen $X(I)]$ at the mRNA level. Significant differences are indicated as follows: ${ }^{*}=p<0.05 ;{ }^{* *}=p<0.01$; and ${ }^{* * *}=p<0.001$. Data are shown as average \pm SD for $n=6$ in biochemical analyses and $n=5$ in real time PCR. 
Figure 2.4. Adipogenic differentiation of SDSCs. The SDSCs from P3.Plastic, P5.Plastic or P5.ECM were incubated in adipogenic induction medium for 14 days. PCM was used to detect adipose vacuoles, which was also confirmed by Oil Red O staining (A). The SDSCs incubated in DMEM with 10\% FBS served as a control and are referred to as either a control for PCM ("P"-Control) or a control for Oil Red O ("O"-Control). All scale bars are $200 \mu \mathrm{m}$. $\operatorname{TaqMan}^{\circledR}$ real time PCR was also used to quantitatively assess relative gene expression of adipogenic markers [LPL (B), PPARY (C), and C/EBPa (D)]. Significant differences are indicated as follows: ${ }^{*}=p<0.05$ and $^{* *}=p<0.01$. Data are shown as average \pm SD for $n=5$ in real time PCR.

Figure 2.5. Osteogenic differentiation of SDSCs. The SDSCs from P3.Plastic, P5.Plastic or P5.ECM were incubated in osteogenic induction medium for 14 days. PCM was used to detect bone nodules, which was also confirmed by Alizarin Red staining (A). The SDSCs incubated in DMEM with 10\% FBS served as a control and are referred to as either a control for PCM ("P"-Control) or a control for Alizarin Red ("A"-Control). All scale bars are $200 \mu \mathrm{m}$. $\operatorname{TaqMan}^{\circledR}$ real time PCR was also used to quantitatively assess relative gene expression of osteogenic markers [OCN (B), SPP1 (C), and Collagen I (D)]. Significant differences are indicated as follows: ${ }^{*}=p<0.05 ;{ }^{* *}=p<0.01$; and ${ }^{* *}=p<0.001$. Data are shown as average \pm SD for $n=5$ in real time PCR. 
Table 2.1. TaqMan ${ }^{\circledR}$ Customized Porcine Marker Gene Primers and Probes

\begin{tabular}{|c|c|c|c|}
\hline Name & Primer/Probe & Sequence (5'-3') & $\begin{array}{l}\text { Genebank } \\
\text { Accession }\end{array}$ \\
\hline \multicolumn{4}{|c|}{ Chondrogenic markers } \\
\hline \multirow{3}{*}{ Aggrecan } & Forward & GCCACTGTTACCGCCACTT & \multirow{3}{*}{ X60107 } \\
\hline & Reverse & CACTGGCTCTCTGCATCCA & \\
\hline & Probe & CTGACCGGGCGACCTG & \\
\hline \multirow{3}{*}{ Col I $\alpha 1$} & Forward & CCGTGCCCTGCCAGATC & \multirow{3}{*}{ AF201723 } \\
\hline & Reverse & CAGTTCTTGATTTCGTCGCAGATC & \\
\hline & Probe & TCGCACAACACATTGC & \\
\hline \multirow{3}{*}{ Col II $\alpha 1$} & Forward & TCCTGGCCTCGTGGGT & \multirow{3}{*}{ AF201724 } \\
\hline & Reverse & GGGATCCGGGAGAGCCA & \\
\hline & Probe & CTCCCCTGGGAAACC & \\
\hline \multirow{3}{*}{ Col X a 1} & Forward & GGCCCGGCAGGTCATC & \multirow{3}{*}{ NM_001005153 } \\
\hline & Reverse & TGGGATGCCTTTTGGTCCTT & \\
\hline & Probe & TCAGACCTGGTTCCCC & \\
\hline \multirow{3}{*}{ Sox 9} & Forward & TGGCAAGGCTGACCTGAAG & \multirow{3}{*}{ AF029696 } \\
\hline & Reverse & GCTCAGCTCGCCGATGT & \\
\hline & Probe & CCCCATCGACTTCCGC & \\
\hline \multicolumn{4}{|c|}{ Adipogenic markers } \\
\hline \multirow{3}{*}{ LPL } & Forward & GATTCATACTTCAGCTGGTCCAACT & \multirow{3}{*}{ X62984 } \\
\hline & Reverse & CTGCСTTCACTCTGATCTTСTCAАT & \\
\hline & Probe & CCAGGGCTGCTCCACC & \\
\hline \multirow{3}{*}{ CEBP/a } & Forward & AAGCGGGTGGAACAACTGA & \multirow{3}{*}{ AF103944.1 } \\
\hline & Reverse & GCAGCTGGCGGAAGATG & \\
\hline & Probe & CCGCAGCGTGTCCAG & \\
\hline \multirow{2}{*}{ PPARr } & Forward & AGGGCGATCTTGACAGGAAAG & \multirow{2}{*}{ AF059245 } \\
\hline & Reverse & АТСTTСТСССАTCATTAAGGAATTCATGTC & \\
\hline
\end{tabular}




\begin{tabular}{|c|c|c|c|}
\hline & Probe & CCACAGACAAATCACC & \\
\hline \multicolumn{4}{|c|}{ Osteogenic markers } \\
\hline \multirow{3}{*}{ OCN } & Forward & CCTGCTGGCCTGGCT & \multirow{3}{*}{ AY150038.1 } \\
\hline & Reverse & CCGGGCAGGGAAGTCA & \\
\hline & Probe & CAGCACGCCCAGCTC & \\
\hline \multirow{3}{*}{ SPP1 } & Forward & CCCAAGGCCATCCTCGTT & \multirow{3}{*}{ X16575 } \\
\hline & Reverse & GTCTCCTGACTGTCСTTCTCTTG & \\
\hline & Probe & CCCAGCGCCTGCACG & \\
\hline Col I $\alpha 1$ & \multicolumn{3}{|c|}{ See chondrogenic markers } \\
\hline
\end{tabular}


Figure 2.1

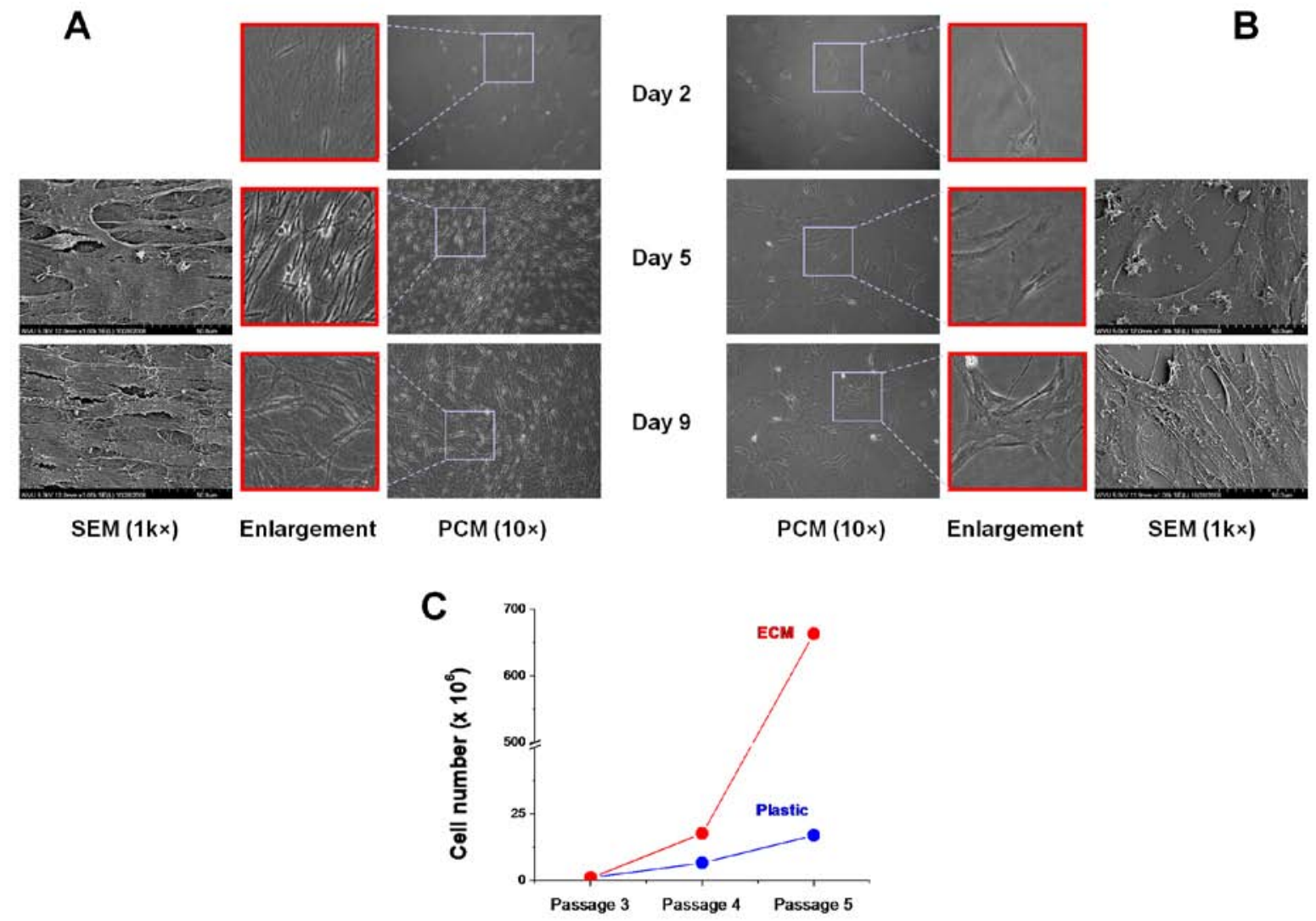

D

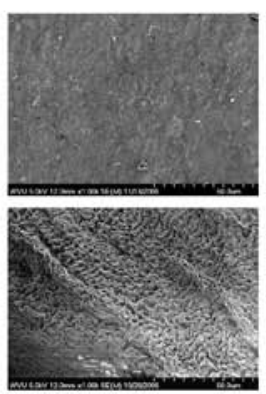

SEM (1k $\times)$

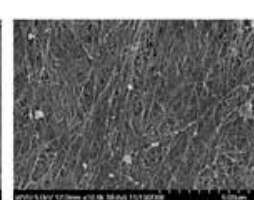

"Stressed" ECM

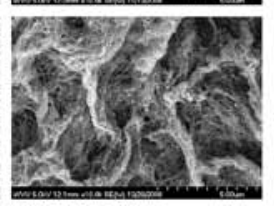

SEM (10k $\times)$
E

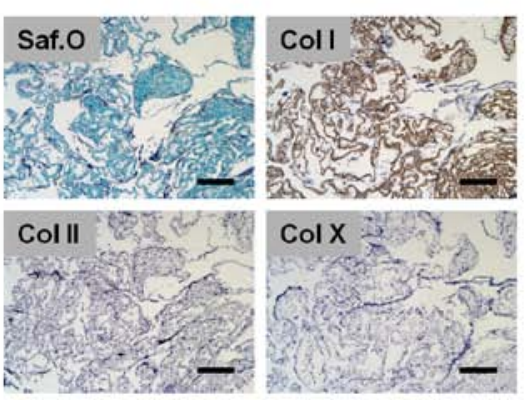


Figure 2.2

A
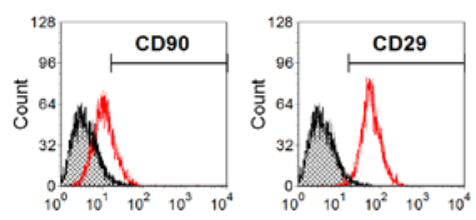

(128)

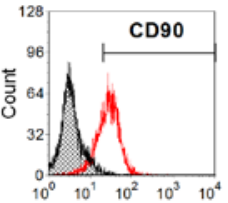

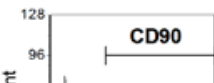

Oे

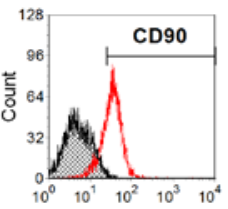

C
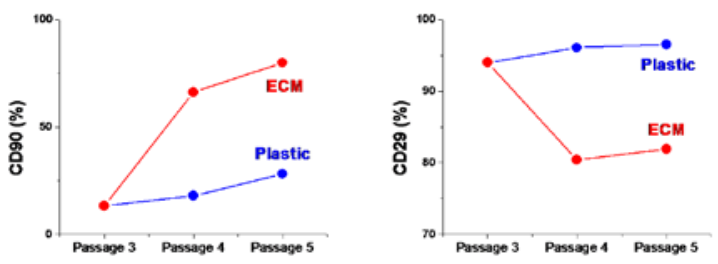

D
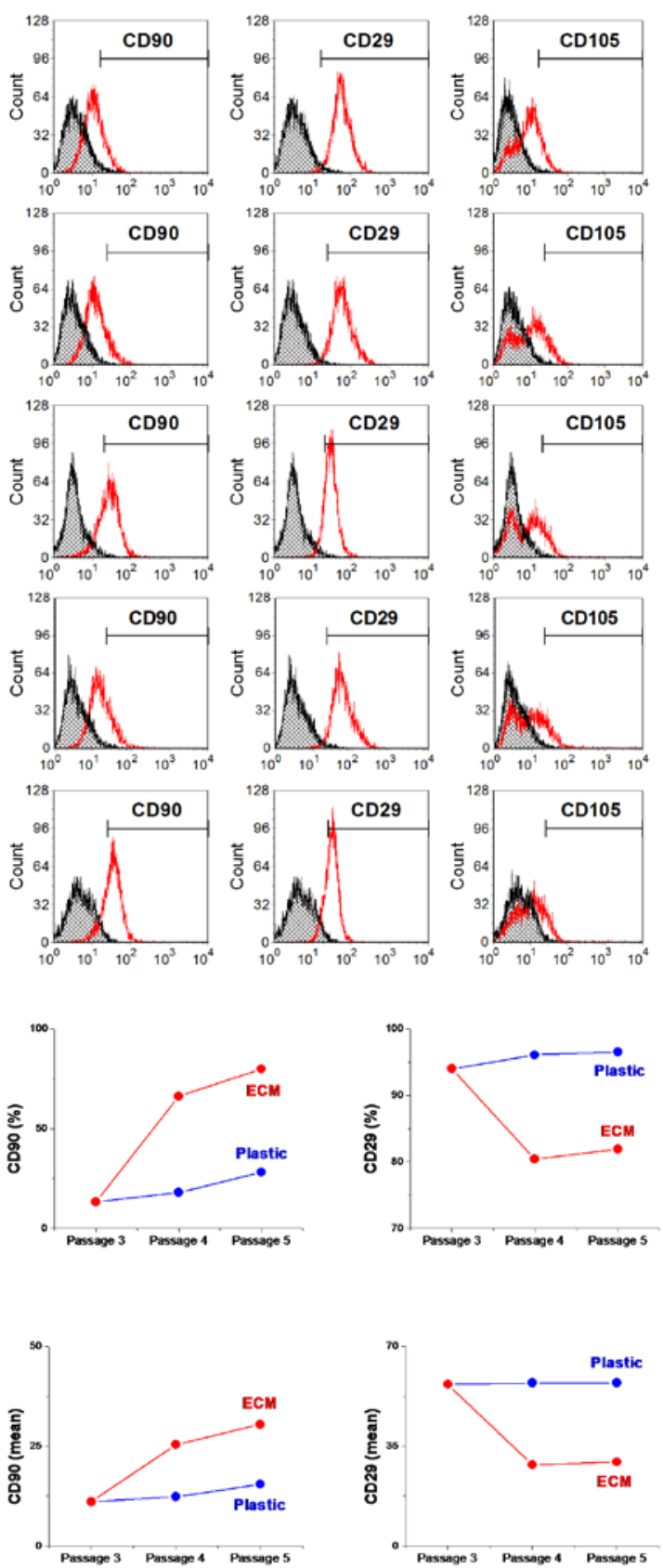

$1 2 8 \longdiv { \quad C D 2 9 }$

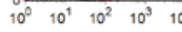

128
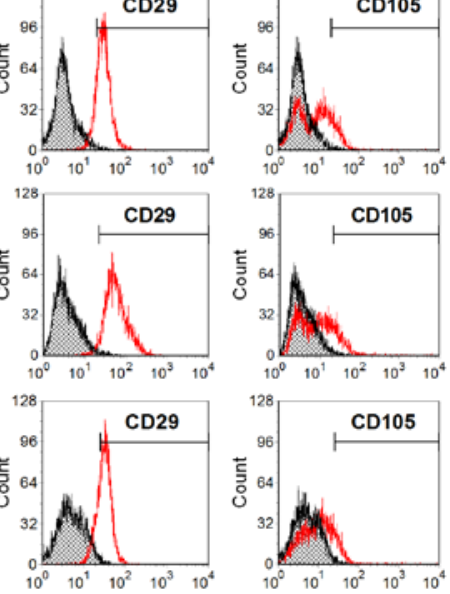

$\stackrel{128}{96} \quad$ CD105

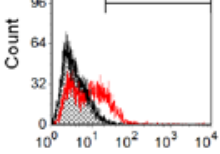

${ }_{96}^{128} \stackrel{\text { CD105 }}{ }$
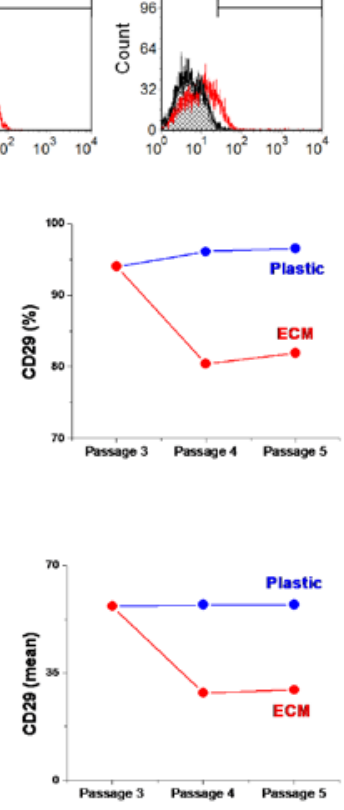

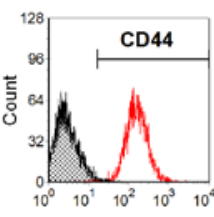

B
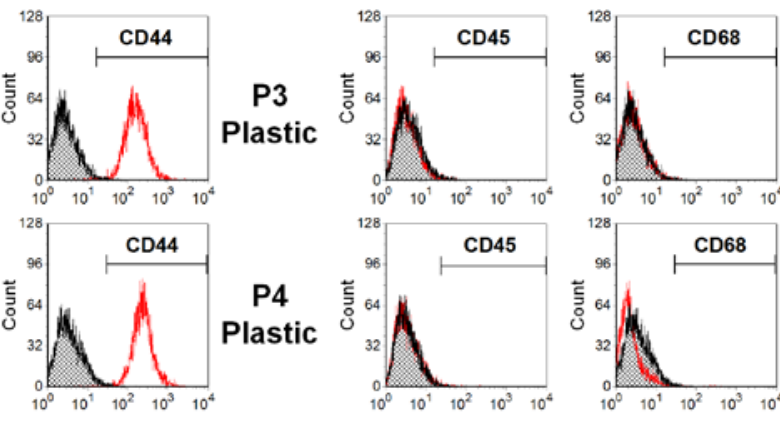

P4
Plastic

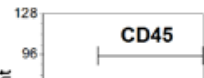

${ }_{96}^{128} \quad$ CD68

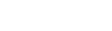

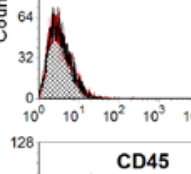

$\sqrt{32} \sqrt{10^{2}}$

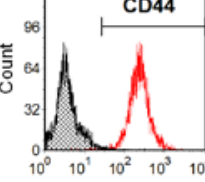

P4
ECM

$128 \quad$ CD44

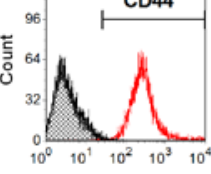

P5
Plastic

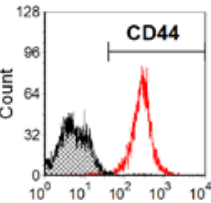

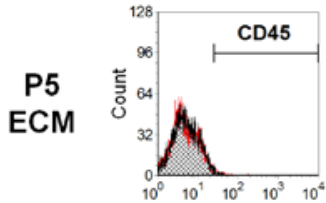
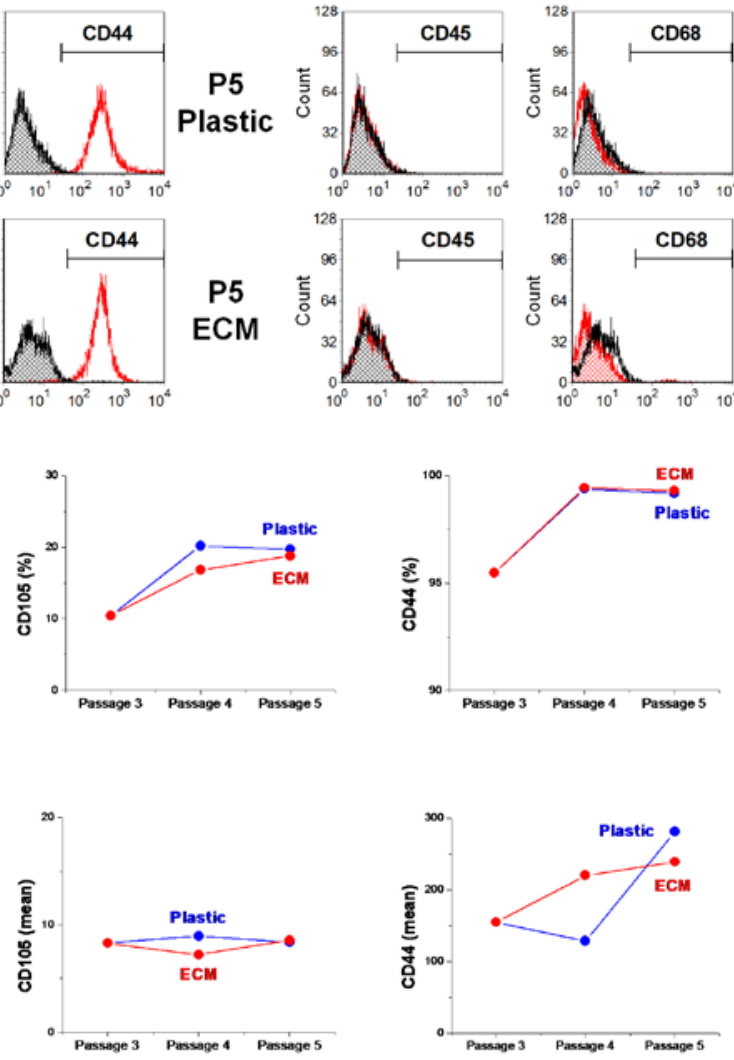
Figure 2.3
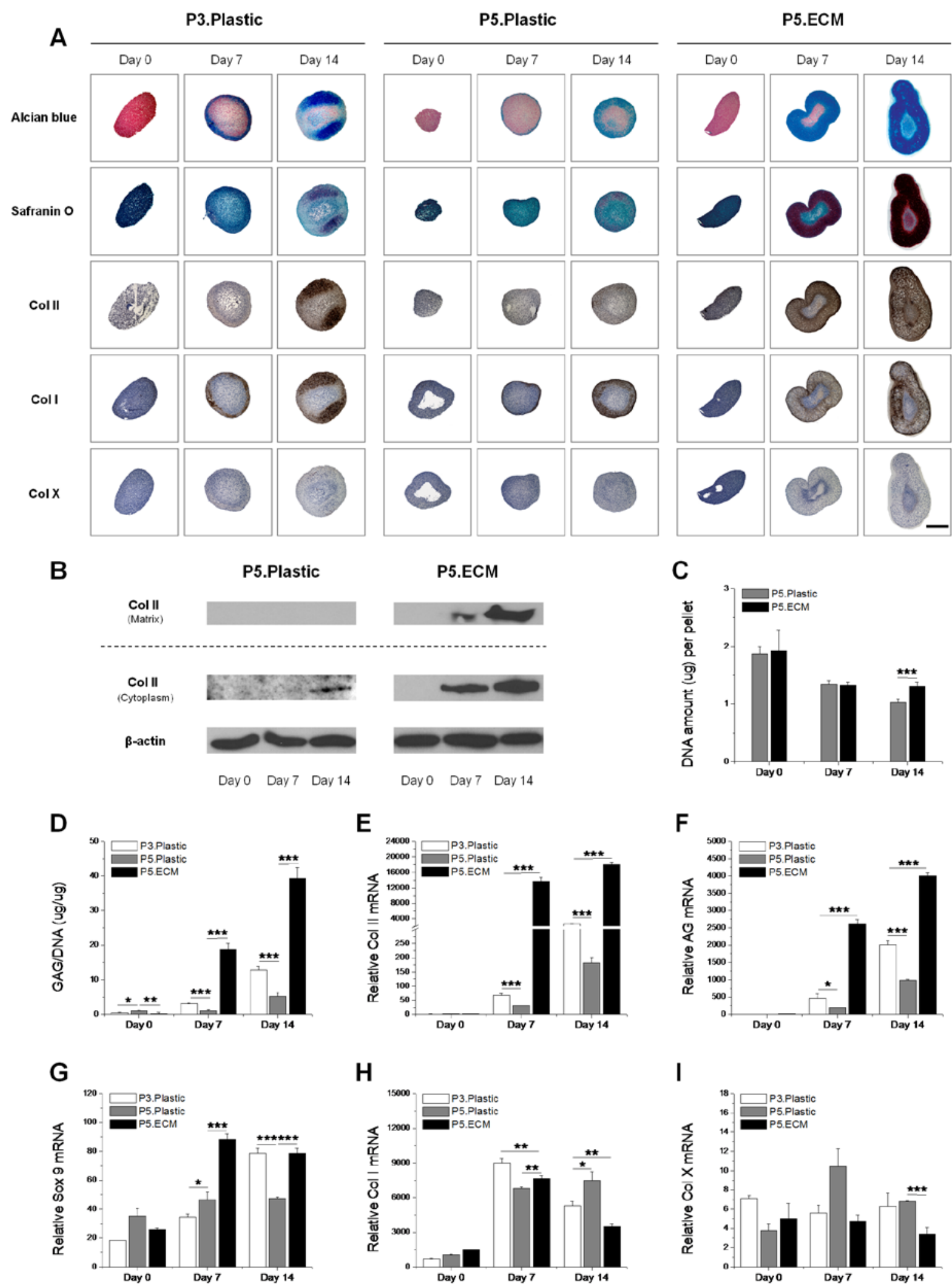
Figure 2.4

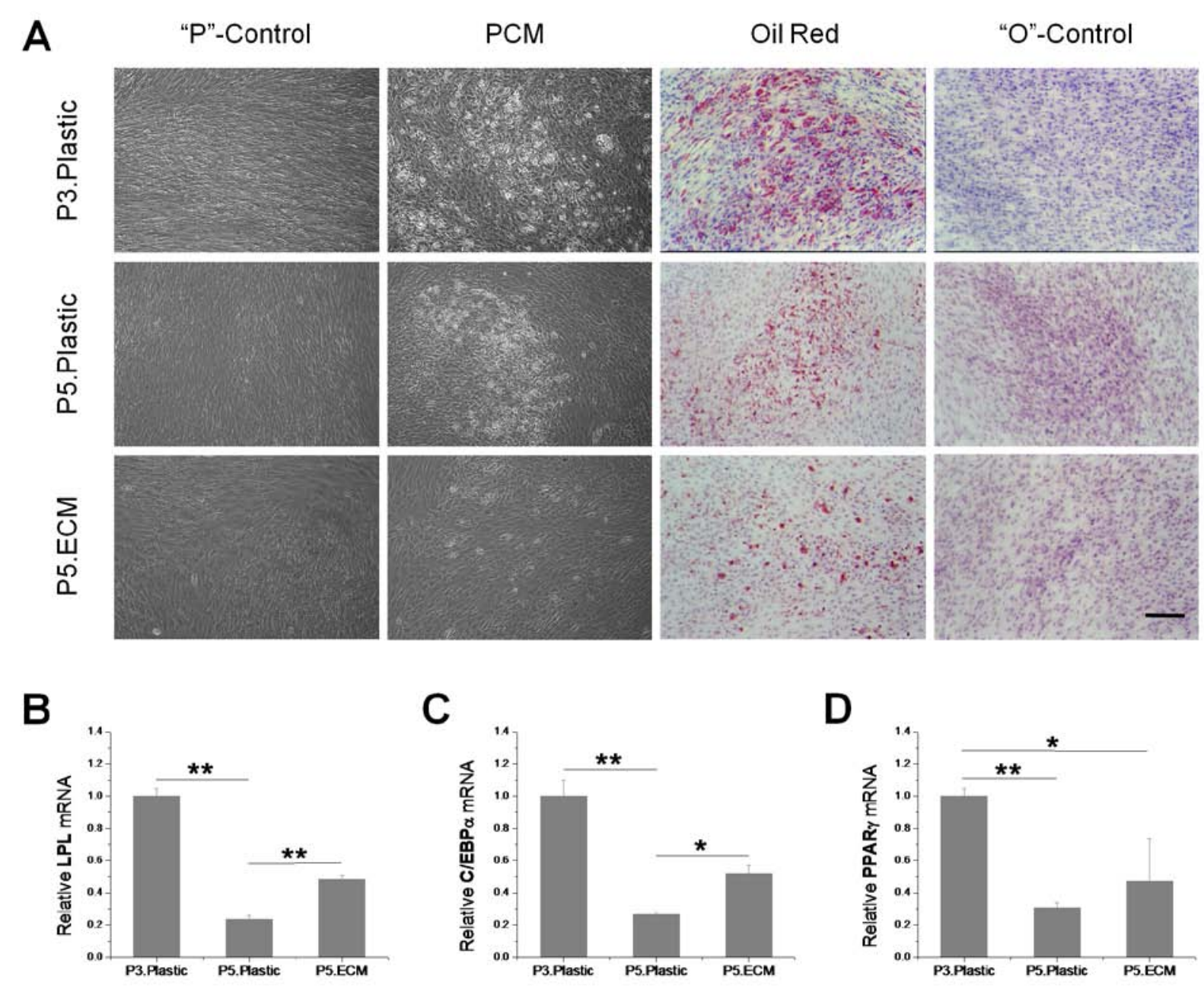




\section{Figure 2.5}

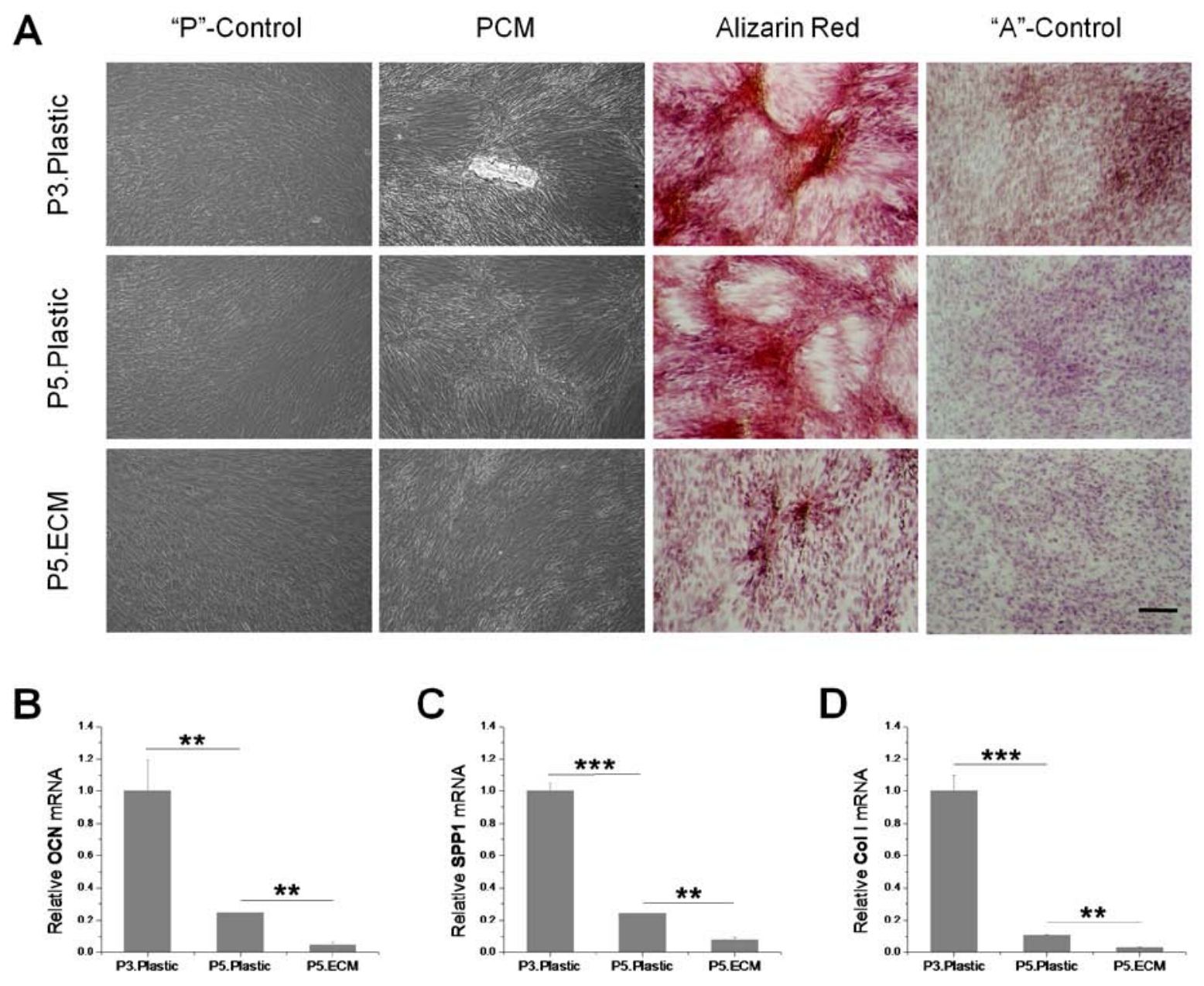




\section{Chapter 3}

In Vitro Three-Dimensional Extracellular Matrix Reprograms Adipose Stem Cells from Infrapatellar Fat Pad toward Chondrogenesis

Fan He B.S. and Ming Pei M.D., Ph.D.

Stem Cell and Tissue Engineering Laboratory, Department of Orthopaedics and Division of Exercise Physiology, West Virginia University, Morgantown, West Virginia 26506

Key words: Adipose stem cell, Extracellular matrix, Cartilage regeneration, Infrapatellar fat pad

Running head: ECM REPROGRAMS ADIPOSE STEM CELLS TOWARD

CHONDROGENESIS 


\begin{abstract}
Objective. To improve the proliferative capacity and chondrogenic differentiation potential of adipose stem cells (ASCs) by large-scale ex vivo expansion on either ASC- or synovium-derived stem cell (SDSC)-derived extracellular matrix (ECM) followed by differentiation in a serum-free chondrogenic medium.
\end{abstract}

Methods. ASCs isolated from porcine infrapatellar fat pad were expanded in plastic flasks coated with ECM deposited by either ASCs or SDSCs for two passages. ASCs grown in non-coated flasks served as a control. The expanded ASCs were evaluated for cell proliferation and intracellular reactive oxygen species (ROS). The cells were centrifuged to form pellets and cultured in a serum-free chondrogenic medium with either $10 \mathrm{ng} / \mathrm{mL}$ transforming growth factor beta 3 (TGF- $\beta 3$ ) or $10 \mathrm{ng} / \mathrm{mL}$ TGF- $\beta 3$ combined with $10 \mathrm{ng} / \mathrm{mL}$ bone morphogenetic protein 6 (BMP-6) for 14 days. The expanded ASCs were also evaluated for their chondrogenic differentiation capacity using histology, immunohistochemistry, biochemistry, and real-time PCR.

Results. The cell number yielded on ECM expansion did not show significant difference in deposition between ASCs and SDSCs and was 6 to 10 times that grown on conventional plastic flasks. ECM-expanded ASCs exhibited a lower ROS level compared to those grown on non-coated flasks. Our histology data showed that typical chondrogenic markers, including type II collagen and glycosaminoglycans, were intensively distributed in the pellets from ECM-expanded ASCs instead of those from flask-grown cells. Our biochemical analysis data indicated that ASCs expanded on ECM, either from ASCs or SDSCs, exhibited a similar chondrogenic index (GAG/DNA), which was significantly higher than that from ASCs grown on plastic flasks. The combination of TGF- $\beta 3$ and BMP-6 increased $36 \%$ more in ASC chondrogenic index than the treatment with TGF- $\beta 3$ alone. Real-time PCR was consistent with the above data. Interestingly, ECM pretreatment also decreased expanded ASC hypertrophy (ALP and MMP13). ECM deposited by either ASCs or SDSCs did not exhibit enhanced adipogenic differentiation of ASCs.

Conclusion. Ex vivo expansion of ASCs on ECM, deposited by either ASCs or SDSCs, had a dramatic increase in cell proliferation and chondrogenic differentiation capacity. The 
expanded cells did not display a similar increase in adipogenesis even on ECM deposited by ASCs. The supplementation of BMP-6 enhanced TGF- $\beta 3$-induced ASC chondrogenesis while inhibiting concomitant hypertrophy. The sequential application of ECM for cell expansion and combined TGF- $\beta 3$ with BMP-6 for chondrogenic differentiation may be a promising approach for ASC-based cartilage tissue engineering and regeneration. 


\section{INTRODUCTION}

Articular cartilage is an avascular tissue with limited capacity for self-repair. Current treatment options for cartilage injuries vary greatly in success rates. Autologous chondrocyte transplantation (ACT) has shown promising results in early clinical studies ${ }^{[1]}$ but, recently, randomized trials indicate little difference in the efficacy of this procedure compared with that of inducing subchondral bone microfracture ${ }^{[2]}$. This approach has also been associated with significant donor-site morbidity and the initiation of osteoarthritic changes in the joint ${ }^{[3]}$, suggesting the need for alternative cell sources for cell-based cartilage repair.

An emerging body of literature suggests that the adult stem cell is a promising cell source for cartilage repair due to its proliferation and multilineage differentiation capacity. Of the stem cells, bone marrow stromal cells (BMSCs) and adipose stem cells (ASCs) are two typical stromal populations isolated from a fatty compartment with similar properties in terms of immunogenicity, growth kinetics, cell senescence, gene transduction efficiency, and surface marker expression ${ }^{[4-6]}$. Despite a better chondrogenic potential, BMSCs also showed a stronger propensity than ASCs toward a hypertrophic chondrocyte phenotype ${ }^{[7-10]}$. Compared to bone marrow with one stem cell in one hundred thousand cell population, the average yield from adipose tissue is in the range of $200,000-290,000$ cells/g of tissue ${ }^{[11]}$, making adipose tissue an abundant and accessible source of stem cells for cell-based therapy.

Despite easy access and abundance, the chondrogenic potential of ASCs from subcutaneous adipose tissue was much weaker than that of BMSCs ${ }^{[12,13]}$. In contrast, ASCs from the infrapatellar fat pad (IPFP) exhibited higher chondrogenic capacity ${ }^{[14,15]}$ and surface zone protein (SZP)-producing phenotypes ${ }^{[16]}$. Like synovial tissue ${ }^{[17]}$, the IPFP also has the potential to self-regenerate after removal from joints ${ }^{[18]}$ and can be obtained arthroscopically by the orthopaedic surgeon with minimal invasion and without causing complications at the donor site. However, synovial tissue in patients with osteoarthritis may be heterogeneous due

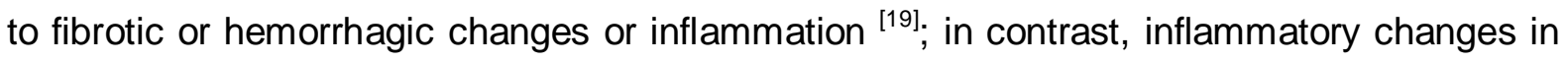
IPFP of the osteoarthritic knee were less frequent. Therefore, the IPFP may be a better cell 
source for superficial zone cartilage tissue engineering for osteoarthritic patients.

The goal of this study was to find an ex vivo expansion approach for the reprogramming of IPFP-derived ASCs toward chondrogenesis. Our recent study suggested that extracellular matrix (ECM) deposited by synovium-derived stem cells (SDSCs) enhanced the seeded SDSC proliferation and chondrogenic differentiation capacity but not adipogenic and osteogenic differentiation ability ${ }^{[20]}$. These results indicate that ECM deposited by a tissue-specific stem cell can provide an in vitro 3D microenvironment for stem cell rejuvenation toward chondrogenesis. In this study, we evaluated the efficiency of IPFP-derived ASCs expanded by ECM, deposited either from ASCs or SDSCs, in order to identify whether the chondrogenic capacity of ASCs will be enhanced after expansion on ECM and whether ECM deposited by different cell types will have a different reprogramming effect. Our study also evaluated whether or not bone morphogenetic protein 6 (BMP-6) would enhance transforming growth factor beta3 (TGF- $\beta 3$ )-induced chondrogenic differentiation but inhibit hypertrophy. 


\section{MATERIALS AND METHODS}

Isolation and culture of ASCs and SDSCs

The protocol was approved by the West Virginia University Animal Care and Use Committee. The IPFP and synovial membrane were harvested from both knees of two 3-month-old minipigs (Marshall BioResources, North Rose, NY) and finely minced into pieces with scapel blades. The IPFP tissue was digested in $0.1 \%$ collagenase P (Roche, Indianapolis, IN) at $37^{\circ} \mathrm{C}$ for $1 \mathrm{~h}$ to release ASCs. The synovial membrane was digested at $37^{\circ} \mathrm{C}$ in $0.1 \%$ trypsin (Roche) for $30 \mathrm{~min}$ and then in $0.1 \%$ collagenase $\mathrm{P}$ (Roche) for $2 \mathrm{~h}$. Both released cells were filtered through 70- $\mu$ m nylon filter (BD Falcon, Bedford, MA) and plated at $3000 \mathrm{cells} / \mathrm{cm}^{2}$ in complete medium [ $\alpha$-minimum essential medium ( $\alpha$-MEM, Invitrogen, Carlsbad, CA) containing 10\% fetal bovine serum (FBS, Atlanta Biologicals, Lawrenceville, GA), $100 \mathrm{U} / \mathrm{ml}$ penicillin, 100 $\mu \mathrm{g} / \mathrm{ml}$ streptomycin and $0.25 \mu \mathrm{g} / \mathrm{ml}$ fungizone (Invitrogen)] at $37^{\circ} \mathrm{C}$ in humidified $5 \% \mathrm{CO}_{2}$ and $21 \% \mathrm{O}_{2}$ incubator. Nonadherent cells were removed by medium change every two days. For negative isolation of SDSCs from primary cultures ${ }^{[21]}$, a cell suspension $\left(10^{7}\right.$ cells $\left./ \mathrm{mL}\right)$ was incubated with $5 \times 10^{7} / \mathrm{mL}$ Dynabeads ${ }^{\circledR}$ M-450 CD14 containing a monoclonal antibody specific for macrophages (Dynal Biotech, Oslo, Norway) at $4^{\circ} \mathrm{C}$ for $1 \mathrm{~h}$. The conjugated cells and the unbound Dynabeads ${ }^{\circledR}$ were collected using the Dynal Magnetic Particle Concentrator ${ }^{\circledR}$ (Dynal Biotech), and the depleted supernatant with SDSCs was saved for further passaging.

\section{Preparation of ASC-ECM and SDSC-ECM}

The procedure of obtaining cell-free ECM was described in our previous study ${ }^{[20]}$. Briefly, 75 $\mathrm{cm}^{2}$ of plastic flasks and 6 -well plates were pretreated with $0.2 \%$ gelatin solution (Sigma, St. Louis, MO) for $1 \mathrm{~h}$ at $3 \pi$, followed by $1 \%$ glutaraldehyde (Sigma) and $1 \mathrm{M}$ ethanolamine (Sigma) sequentially for 30 min each at room temperature. Passage 3 ASCs and SDSCs were seeded on pretreated plastic flasks and 6-well plates at $3000 \mathrm{cells} / \mathrm{cm}^{2}$ in complete medium for 7 days. When cells reached $90 \%$ confluence, culture medium was supplemented with $50 \mu \mathrm{g} / \mathrm{mL}$ L-ascorbic acid phosphate (Wako Chemicals USA Inc., Richmond, VA) for an additional 8 days. The ECMs deposited by ASCs and SDSCs were incubated with $0.5 \%$ Triton X-100 (Sigma) containing 20 mM ammonium hydroxide (Sargent-Welch, Skokie, IL) at 
$37^{\circ} \mathrm{C}$ for $5 \mathrm{~min}$.

Culture of ASCs on Plastic and ECM

Passage 1 ASCs were cultured in complete medium for two passages in three conditions: plastic flasks (Plastic), flasks coated with ASC-derived ECM (AECM), and flasks coated with SDSC-derived ECM (SECM). The culture period for each passage was seven days and the medium was changed every 3 days. At each passage, cell number was counted using a counting hemacytometer (Hausser Scientific, Horsham, PA) for cell proliferative rate.

\section{Measurement of intracellular reactive oxygen species (ROS)}

Intracellular ROS generation was measured with 2',7'-dichlorofluorescein-diacetate (DCFH-DA) (Sigma). In brief, $0.2 \times 10^{6}$ of the detached ASCs were washed with PBS and incubated in $10 \mu \mathrm{M}$ DCFH-DA at $37^{\circ} \mathrm{C}$ for $10 \mathrm{~min}$. After washing twice with PBS, the fluorescence from',72 -dichlorofluorescein (DCF) was analyzed by a dual-laser fluorescence-activated cell sorting (FACS) Calibur CytoFluorimeter (BD Biosciences, Bedford, MA) using FCS Express Software Package (De Novo Software, Los Angeles, CA) and 20000 events of each sample were analyzed.

\section{Chondrogenic differentiation of the expanded ASCS}

$3 \times 10^{5}$ of ASCs at passage 1 and passage 3 cultured on Plastic, AECM and SECM were centrifuged at $500 \mathrm{~g}$ for $5 \mathrm{~min}$ to form a pellet in a 15-ml polypropylene tube. After $24 \mathrm{~h}$ of incubation, the pellets were incubated in a serum-free chondrogenic medium [high-glucose DMEM, $40 \mu \mathrm{g} / \mathrm{ml}$ proline (Sigma), $100 \mathrm{nM}$ dexamethasone (Sigma), $100 \mathrm{U} / \mathrm{ml}$ penicillin, 100 $\mu \mathrm{g} / \mathrm{ml}$ streptomycin, $0.1 \mathrm{mM}$ ascorbic acid-2-phosphate and ITS TM Premix (6.25 $\mu \mathrm{g} / \mathrm{mL}$ insulin, $6.25 \mu \mathrm{g} / \mathrm{mL}$ transferrin, $6.25 \mu \mathrm{g} / \mathrm{mL}$ selenous acid, $5.35 \mu \mathrm{g} / \mathrm{mL}$ linoleic acid and $1.25 \mu \mathrm{g} / \mathrm{mL}$ bovine serum albumin, from BD Biosciences] with the supplementation of $10 \mathrm{ng} / \mathrm{ml}$ of TGF- $\beta 3$ (PeproTech Inc., Rocky Hill, NJ) alone or $10 \mathrm{ng} / \mathrm{mL}$ of TGF- $\beta 3$ combined with $10 \mathrm{ng} / \mathrm{ml}$ of BMP-6 (PeproTech Inc). The pellets were collected at days 0, 7 and 14 for histology, immunohistochemistry, biochemistry, and real-time PCR. 
Adipogenic differentiation of the expanded ASCs

Passage 3 ASCs were cultured on Plastic, AECM, or SECM in 6-well plates until 95\% confluence, and the complete medium was switched to adipogenic differentiation medium [low glucose DMEM (Invitrogen), 10\% FBS, $100 \mathrm{U} / \mathrm{ml}$ penicillin, $100 \mu \mathrm{g} / \mathrm{ml}$ streptomycin, 10 ${ }^{-6}$ M dexamethasone, $0.5 \mathrm{mM}$ isobutyl-1-methy xanthine (Sigma), $100 \mu \mathrm{M}$ indomethacin (Sigma) and $10 \mu \mathrm{g} / \mathrm{mL}$ insulin (Sigma)] for an additional 14 days. The cultured cells were fixed in $4 \%$ paraformaldehyde (Sigma), stained with Oil Red O solution (Diagnostic Biosystems, Pleasanton, CA) and counterstained with hematoxylin (Vector Laboratories Inc., Burlingame, CA). The expanded cells were also collected for quantitative evaluation of adipogenic gene expression using real-time PCR.

\section{Histology and Immunohistochemistry}

The pellets $(n=2)$ were fixed at $4^{\circ} \mathrm{C}$ in $4 \%$ paraformaldehyde, embedded in paraffin blocks and cut into $5-\mu m$ thick sections. Safranin O solution (Sigma) was used to stain sulfated glycosaminoglycans (GAGs). For immunohistochemical analysis, the sections were immunolabeled with primary antibodies against collagen II (II-II6B3, DSHB, Iowa City, IA), collagen I (Abcam, Cambridge, MA), and collagen X (Sigma), followed by the secondary antibody of biotinylated horse anti-mouse IgG (Vector). Immunoactivity was detected using Vectastain $A B C$ reagent (Vector) with 3,3'-diaminobenzidine (DAB) as a substrate.

\section{Biochemical analysis}

The pellets $(n=5)$ were digested for $4 \mathrm{~h}$ at $60^{\circ} \mathrm{C}$ with $125 \mu \mathrm{g} / \mathrm{mL}$ papain in PBE buffer (100 $\mathrm{mmol} / \mathrm{L}$ phosphate, $10 \mathrm{mmol} / \mathrm{L}$ EDTA, $\mathrm{pH}$ 6.5) containing $10 \mathrm{mmol} / \mathrm{L}$ cysteine, by using $100 \mu \mathrm{L}$ enzyme per sample. DNA amount in the papain digestion was measured using the QuantiT ${ }^{\mathrm{TM}}$ PicoGreen ${ }^{\circledR}$ dsDNA assay kit (Invitrogen) with a CytoFluor ${ }^{\circledR}$ Series 4000 (Applied Biosystems, Foster City, CA). GAG was measured using dimethylmethylene blue dye and a Spectronic ${ }^{\mathrm{TM}}$ BioMate $^{T M} 3$ Spectrophotometer (Thermo Scientific, Milford, MA) with bovine chondroitin sulfate (Sigma) as a standard. 
The total mRNA from pellets $(n=4)$ was extracted using TRIzol ${ }^{\circledR}$ (Invitrogen) and $1 \mu \mathrm{g}$ of mRNA was converted to cDNA with a High-Capacity cDNA Archive Kit (Applied Biosystems). Chondrogenic marker genes [aggrecan, types I and II collagen, and SRY (sex determining region Y)-box 9 (Sox9)], hypertrophy marker genes [type $X$ collagen and matrix metalloproteinase 13 (MMP13)], and adipogenic marker genes [lipoprotein lipase (LPL), peroxisome proliferator activated receptor Y (PPARY), and CCAAT enhancer binding protein $\alpha(\mathrm{C} / \mathrm{EBP} \alpha)]$ were customized by Applied Biosystems as part of the Custom TaqMan ${ }^{\circledR}$ Gene Expression Assays. Eukaryotic 18S rRNA (Assay ID HS99999901_s1 ABI) was carried out as the endogenous control gene. Real-time PCR was performed with the iCycler $\mathrm{iQ}^{\mathrm{TM}}$ Multi Color RT-PCR Detection and calculated by computer software (Perkin-Elmer, Waltham, MA).

Relative transcript levels were calculated as $\mathrm{X}=2^{-\Delta \Delta \mathrm{Ct}}$, in which $\Delta \Delta \mathrm{Ct}=\Delta \mathrm{E}-\Delta \mathrm{C}, \Delta \mathrm{E}=\mathrm{Ct}_{\mathrm{exp}}-\mathrm{Ct}_{18 \mathrm{~s}}$, and $\Delta \mathrm{C}=\mathrm{Ct}_{\mathrm{ct1}}-\mathrm{Ct}_{18 \mathrm{~s}}$.

\section{Statistical Analysis}

The Kruskal-Wallis test was used to test for significant differences among all groups and the Mann-Whitney $U$ test was used for pairwise comparison. All the statistical analysis was performed with SPSS 13.0 statistical software (SPSS Inc., Chicago, IL). P values less than 0.05 were considered statistically significant. 


\section{RESULTS}

ECM expansion enhanced ASC proliferation and sustained stem cell morphology

(Figure 3.1A) Passage 1 ASCs were expanded on Plastic, AECM, or SECM for two passages. In regard to cell morphology, ASCs grown on Plastic appeared large and flattened while ASCs plated on ECM became smaller and spindle-like and overlapped with time in a 3D format. Intriguingly, ASCs grown on AECM or SECM exhibited similar cell morphology and underwent directional migration along the ECM fibrils whereas the cells on Plastic grew in a random direction. (Figure 3.1B) During a seven-day incubation, the proliferative rate of ASCs on Plastic dropped with each passage, with a 4.53-fold increase at passage 2 and a 7.47 -fold increase at passage 3 compared to a 15.47 -fold increase at passage 1 . On the contrary, ASCs plated on AECM and SECM exhibited a robust and stable proliferative ability, with a 43.73-fold increase at passage 2 and a 44.8-fold increase at passage 3 for ASCs expanded on AECM and a 43.73-fold increase at passage 2 and a 48.8-fold increase at passage 3 for ASCs expanded on SECM. There was no significant difference in the cell yield number in two different ECMs.

(Figure 3.1C) In order to determine whether ECM expansion could decrease cell stress, we examined the intracellular levels of ROS accumulation in ASCs cultured in Plastic, AECM, or SECM using flow cytometry. The ASCs cultured in Plastic indeed showed a higher level of DCF fluorescence compared with those expanded in AECM or SECM. (Figure 3.1D) The mean fluorescent intensity (MFI) of ROS in ASCs in Plastic was $148 \pm 6$, which was 2.4-fold that in $A E C M$ and 3.5-fold that in SECM, indicating that ECM effectively reduced the generation or accumulation of the intracellular ROS in ASCs.

\section{ECM expansion enhanced ASC chondrogenic capacity}

After expansion on Plastic, AECM, and SECM, ASCs were cultured in a serum-free pellet system supplemented with $10 \mathrm{ng} / \mathrm{mL}$ of TGF- $\beta 3$ to evaluate the effect of expansion approach on ASC chondrogenic capacity. (Figure 3.2) Compared to nondetectable staining at day 0 , ASC pellets from P1.Plastic or P3.Plastic were barely positive for sulfated GAGs and type II 
collagen at day 7 despite a gradually positive staining on the periphery of pellets at day 14 . The pellets from P3.AECM and P3.SECM, however, were intensively positive for sulfated GAGs and type II collagen even at day 7 and dramatically improved in range and density after incubation in chondrogenic medium for 14 days.

(Figure 3.3) At the protein level, ASCs from P3.Plastic yielded pellets with a lower chondrogenic index (GAG/DNA) compared with that from P1.Plastic. In contrast, ASCs from P3.AECM and P3.SECM yielded pellets with not only a sustained cell number but also a greatly enhanced chondrogenic index. There was no significant difference in chondrogenic index for ASCs expanded on AECM or SECM. (Figure 3.4) At the mRNA level, ASCs from P3.AECM or P3.SECM yielded pellets with higher mRNA levels in type II collagen (at days 7 and 14), aggrecan (at days 7 and 14), and Sox9 (at day 7) than that from P1.Plastic and P3.Plastic. Similar to protein expression, there was no significant difference in the above chondrogenic marker genes for ASCs expanded on AECM or SECM.

BMP-6 increased TGF-B3-induced ASC chondrogenic differentiation but inhibited hypertrophy

(Figure 3.2) Compared to the expanded ASCs on ECM, the combined treatment with TGF- $\beta 3$ and BMP-6 yielded pellets from Plastic-expanded ASCs with more intense sulfated GAGs and type II collagen than the treatment with TGF- $\beta 3$ alone. Intriguingly, the expanded ASCs on ECM yielded pellets with less type I collagen stain when the pellets were treated with TGF- $\beta 3$ combined with BMP-6 than that with TGF- $\beta 3$ alone. No type $X$ collagen was detectable in either Plastic- or ECM-expanded ASCs. (data not shown)

(Figure 3.3) The combination of TGF- $\beta 3$ with BMP-6 maintained cell number in ASC pellets compared to treatment with TGF- $\beta 3$ alone, especially after a 14-day incubation. More importantly, the combined treatment significantly increased ASC chondrogenic index in all groups except P3.Plastic compared to treatment with TGF- $\beta 3$ alone. (Figure 3.4\&3.5) Consistent with histology data, the combined treatment significantly decreased mRNA levels of type I collagen, type X collagen, and MMP-13 compared to treatment with TGF- $\beta 3$ alone. 
ECM expansion delayed the decrease of ASC adipogenic capacity

(Figure 3.6A) ASCs from P1.Plastic exhibited more Oil Red O stained spots than the other three groups. (Figure 3.6B) As an early marker gene of adipogenesis, the level of LPL mRNA was the highest in P1.Plastic with only $5.6 \%$ in P3.Plastic, $18.7 \%$ in P3.AECM, and $27.5 \%$ in P3.SECM. (Figure 3.6C,D) PPARY and C/EBPa are the transcription factors involved in adipogenesis, both of which showed a similar tendency as LPL mRNA. 


\section{DISCUSSION}

ASCs are increasingly becoming a promising stem cell source for the treatment of cartilage defects. However, it is still a big challenge to establish an in vitro microenvironment for reprogramming autologous ASCs toward chondrogenic differentiation. In this study, we found that ECM enhanced ASC proliferation, maintained stem cell morphology and lowered ASC intracellular ROS level; ECM-expanded ASCs exhibited a decreased level of intracellular ROS; and the expansion on ECM enhanced ASC chondrogenic differentiation capacity. Interestingly, there was no significant difference in ASC proliferation and differentiation using two different ECMs deposited by either ASCs or SDSCs. Our findings also suggested that BMP-6 increased TGF- $\beta 3$-induced ASC chondrogenic differentiation but inhibited concomitant hypertrophy. Surprisingly, we did not find that the expanded ASCs exhibited an enhanced adipogenic capacity, regardless of expansion on ECM deposited by ASCs or SDSCs.

Plenty of stem cells are needed for stem cell-based tissue engineering and regeneration. Adult tissues contain unique local niches with tissue-specific stem cells; however, ex vivo expansion on conventional plastic flasks results in stem cell senescence, indicated by the Ioss of proliferation and differentiation capacity. In this study, we found that ECM expansion could decrease ASC intracellular ROS level, indicating that ROS associated cell stress may play a major role in monolayer culture on plastic flasks; this could be prevented by expansion on 3D ECM. Due to the 3D growth format on ECM, expanded ASCs grew much faster and were more orderly than 2D growth on plastic flasks. Moreover, ECM deposited by stem cells, either SDSCs or ASCs, could maintain the expanded ASC stem cell characteristics (small with spindle-shape). The connection between stem cell size and function is consistent with bone marrow derived MSCs, which have also been shown to possess more "stemness" when the MSCs are small and rapidly dividing ${ }^{[22]}$. This result is also in agreement with reports from us and other investigators. We found ECM deposited by SDSCs could dramatically enhance seeded SDSC proliferation ${ }^{[20]}$. Chen et al. demonstrated that ECM deposited by BMSCS enhanced plated BMSC expansion ${ }^{[23]}$. These cell-derived matrices appear to more closely 
approximate native tissue ${ }^{[24]}$ rather than the "reconstituted" matrices (such as 3D pure collagen gels and Matrigel matrices ${ }^{[25]}$ ). As noted previously ${ }^{[24,26,27]}$, cells within this in vivo-like 3D environment developed an elongated shape and had reduced lamella, confined predominantly to one of the cell ends. In contrast, control fibroblasts grown on fibronectin-coated surfaces had a flattened and triangular shape with an increased number of peripheral lamellae. No significant difference was observed in ASC proliferation after expansion on ECM deposited by either ASCs or SDSCs, indicating that the stimulatory effect of the matrix is most probably due to its three-dimensionality, rather than to its composition, as flattening of the matrix by mechanical force abolishes the formation of 3D matrix adhesions and ERK-stimulated cell proliferation ${ }^{[24,28]}$.

In our previous study, expanded SDSCS on SDSC-derived ECM exhibited a robust chondrogenic differentiation potential. Since SDSCs are considered a tissue-specific stem cell for chondrogenesis, we proposed that the chondrogenic potential of ASCs would be improved after expansion on ECM deposited by SDSCs instead of ASCs while the adipogenic potential of ASCs would be improved after expansion on ECM deposited by ASCs. Surprisingly, ASCs expanded on ECM deposited from either SDSCs or ASCs developed a similar enhanced chondrogenic differentiation capacity, indicating that 3D ECM may induce sustained activation of the extracellular signal-activated kinase 1/2 (ERK1/2) via Src/Ras/Raf signaling pathway ${ }^{[26]}$ and the high level of phosphorylated ERK1/2 could be responsible for the improved chondrogenic differentiation potential of ASCs. This signal transduction pathway needs to be further demonstrated.

Our study also found that supplementation of BMP-6 enhanced TGF- $\beta$-induced ASC chondrogenesis but inhibit hypertrophy, which is in agreement with reports from other investigators ${ }^{[29,30]}$. Although TGF- $\beta$ can inhibit chondrocyte hypertrophy ${ }^{[31]}$, perhaps the chondrogenic differentiation potential of ASCs treated by ECM was very robust, and the level of TGF- $\beta 3$ used in this study was insufficient to completely stop further development into hypertrophy. Despite the fact that BMP-6 combined with TGF- $\beta$ results in hypertrophy/endochondral ossification pathway in BMSCs ${ }^{[32,33,34]}$, BMP-6 alone is a strong 
chondrogenic inducer and also acts as a hypertrophy inhibitor in ASCs ${ }^{[29]}$. This was in agreement with our results that supplementation of BMP-6 enhanced ASC chondrogenic but inhibited hypertrophic differentiation. The underlying mechanism may be associated with the reduced expression of BMP-2, -4 , and -6 mRNA and no expression of TGF- $\beta$-receptor-1 protein in ASCs, which BMSCs have. BMP-6 treatment induced TGF $\beta$-receptor-1 expression and combined application of TGF- $\beta$ and BMP-6 eliminated the reduced chondrogenic potential of ASCs inducing a gene expression profile similar to differentiated BMSCs. ${ }^{[30]}$

The decreased adipogenic and osteogenic differentiation (data not shown) of ASCs culturing on ASC- and SDSC-derived ECMs could be correlated with the effect of suppressing "spontaneous" differentiation. ECM was proven to modulate the activity of growth factors, interact with cell surface receptors to prevent activation, and absorb growth factors from serum $^{[23,35-37]}$. Expansion of MSCs on ECM not only increased the proliferative rate but also suppressed "spontaneous" differentiation and maintained stem cell properties. However, the molecular mechanism that ECM used to interact with cells and improve the proliferation and chondrogenic potential still needs to be investigated.

In summary, our study proved that the utilization of ECM on ASC ex vivo expansion was able to dramatically increase the cell proliferative rate and improve the chondrogenic differentiation potential. The combination of TGF- $\beta 3$ and BMP-6 promoted chondrogenic induction but suppress hypertrophic gene expression. The large-scale cell number of ADSCs with significant chondrogenic potential will benefit cartilage repair and tissue regeneration in the future. 


\section{REFERENCES}

1. Brittberg M, Lindahl A, Nilsson A, Ohlsson C, Isaksson O, Peterson L. Treatment of deep cartilage defects in the knee with autologous chondrocyte transplantation. $\mathrm{N}$ Engl J Med 1994;331:889-95.

2. Knutsen G, Engebretsen L, Ludvigsen TC, Drogset JO, Grontvedt T, Solheim E, Strand T, Roberts S, Isaksen V, Johansen O. Autologous chondrocyte implantation compared with microfracture in the knee: a randomized trial. J Bone Joint Surg Am 2004;86:455-64.

3. Lee CR, Grodzinsky AJ, Hsu HP, Martin SD, Spector M. Effects of harvest and selected cartilage repair procedures on the physical and biochemical properties of articular cartilage in the canine knee. J Orthop Res 2000;18:790-9.

4. De Ugarte DA, Morizono K, Elbarbary A, Alfonso Z, Zuk P, Zhu M, Dragoo J, Ashjian P, Thomas B, Benhaim P, Chen I, Fraser J, Hedrick M. Comparison of multi-lineage cells rom human adipose tissue and bone marrow. Cells Tissues Organs 2003;174:101-9.

5. Katz AJ, Tholpady A, Tholpady SS, Shang H, Ogle RC. Cell surface and transcriptional characterization of human adipose-derived adherent stromal (hADAS) cells. Stem Cells 2005;23:412-23.

6. Puissant B, Barreau C, Bourin P, Clavel C, Corre J, Bousquet C, Taureau C, Cousin B, Abbal M, Laharrague P, Penicaud L, Casteilla L, Blancher A. Immunomodulatory effect of human adipose tissue-derived adult stem cells: comparison with bone marrow mesenchymal stem cells. Br J Haematol 2005;129:118-29.

7. Diekman BO, Rowland CR, Caplan AI, Lennon D, Guilak F. Chondrogenesis of adult stem cells from adipose tissue and bone marrow: Induction by growth factors and cartilage derived matrix. Tissue Engineering Part A. 2010;16:523-33.

8. Huang JI, Kazmi N, Durbhakula MM, Hering TM, Yoo JU, Johnstone B. Chondrogenic potential of progenitor cells derived from human bone marrow and adipose tissue: a patient-matched comparison. J Orthop Res 2005;23:1383-9.

9. Liu TM, Martina M, Hutmacher DW, Hui JH, Lee EH, Lim B. Identification of common pathways mediating differentiation of bone marrow- and adipose tissue-derived human mesenchymal stem cells into three mesenchymal lineages. Stem Cells 
$2007 ; 25: 750-60$.

10. Mehlhorn AT, Niemeyer P, Kaiser S, Finkenzeller G, Stark GB, Südkamp NP, Schmal H. Differential expression pattern of extracellular matrix molecules during chondrogenesis of mesenchymal stem cells from bone marrow and adipose tissue. Tissue Eng 2006;12:2853-62.

11. van Harmelen V, Skurk T, Röhrig K, Lee YM, Halbleib M, Aprath-Husmann I, Hauner $\mathrm{H}$. Effect of BMI and age on adipose tissue cellularity and differentiation capacity in women. Int J Obes Relat Metab Disord 2003;27:889-95.

12. Sakaguchi Y, Sekiya I, Yagishita K, Muneta T. Comparison of human stem cells derived from various mesenchymal tissues: superiority of synovium as a cell source. Arthritis Rheum 2005;52:2521-9.

13. Winter A, Breit S, Parsch D, Benz K, Steck E, Hauner H, Weber RM, Ewerbeck V, Richter W. Cartilage-like gene expression in differentiated human stem cell spheroids: a comparison of bone marrow-derived and adipose tissue-derived stromal cells. Arthritis Rheum 2003;48:418-29.

14. Khan WS, Adesida AB, Hardingham TE. Hypoxic conditions increase hypoxia-inducible transcription factor 2alpha and enhance chondrogenesis in stem cells from the infrapatellar fat pad of osteoarthritis patients. Arthritis Res Ther 2007;9:R55.

15. Khan WS, Tew SR, Adesida AB, Hardingham TE. Human infrapatellar fat pad-derived stem cells express the pericyte marker 3G5 and show enhanced chondrogenesis after expansion in fibroblast growth factor-2. Arthritis Res Ther 2008;10:R74.

16. Lee SY, Nakagawa T, Reddi AH. Mesenchymal progenitor cells derived from synovium and infrapatellar fat pad as a source for superficial zone cartilage tissue engineering: analysis of superficial zone protein/lubricin expression. Tissue Eng Part A 2010;16:317-25.

17. Bentley G, Kreutner A, Ferguson AB. Synovial regeneration and articular cartilage changes after synovectomy in normal and steroid-treated rabbits. J Bone Joint Surg Br 1975;57:454-62.

18. Ogilvie-Harris DJ, Giddens J. Hoffa's disease: arthroscopic resection of the 
infrapatellar fat pad. Arthroscopy 1994;10:184-7.

19. Loeuille D, Chary-Valckenaere I, Champigneulle J, Rat AC, Toussaint F, Pinzano-Watrin A, Goebel JC, Mainard D, Blum A, Pourel J, Netter P, Gillet P. Macroscopic and microscopic features of synovial membrane inflammation in the osteoarthritic knee: correlating magnetic resonance imaging findings with disease severity. Arthritis Rheum 2005;52:3492-501.

20. He F, Chen X, Pei M. Reconstruction of an in vitro tissue-specific microenvironment to rejuvenate synovium-derived stem cells for cartilage tissue engineering. Tissue Eng Part A 2009;15:3809-21.

21. Pei M, He F, Vunjak-Novakovic G. Synovium-derived stem cell-based chondrogenesis. Differentiation 2008;76:1044-56.

22. Sekiya I, Larson BL, Smith JR, Pochampally R, Cui JG, Prockop DJ. Expansion of human adult stem cells from bone marrow stroma: conditions that maximize the yields of early progenitors and evaluate their quality. Stem Cells 2002;20:530-41.

23. Chen XD, Dusevich V, Feng JQ, Manolagas SC, Jilka RL. Extracellular matrix made by bone marrow cells facilitates expansion of marrow-derived mesenchymal progenitor cells and prevents their differentiation into osteoblasts. J Bone Miner Res 2007;22:1943-56.

24. Cukierman E, Pankov R, Stevens DR, Yamada KM. Taking cell-matrix adhesions to the third dimension. Science 2001;294:1708e12.

25. Grinnell F. Fibroblast-collagen-matrix contraction: growth-factor signaling and mechanical loading. Trends Cell Biol 2000;9:362e5.

26. Damianova R, Stefanova N, Cukierman E, Momchilova A, Pankov R. Three-dimensional matrix induces sustained activation of ERK1/2 via Src/Ras/Raf signaling pathway. Cell Biol Int 2008;32(2):229-234.

27. Pankov R, Endo Y, Even-Ram S, Araki M, Clark K, Cukierman E, Matsumoto K, Yamada KM. A Rac switch regulates random versus directionally persistent cell migration. J Cell Biol 2005;170:793-802.

28. Cukierman E, Pankov R, Yamada KM. Cell interactions with three-dimensional matrices. Curr Opin Cell Biol 2002;14:633e9. 
29. Estes BT, Wu AW, Guilak F. Potent induction of chondrocytic differentiation of human adipose-derived adult stem cells by bone morphogenetic protein 6 . Arthritis Rheum 2006;54:1222-32.

30. Hennig T, Lorenz H, Thiel A, Goetzke K, Dickhut A, Geiger F, Richter W. Reduced chondrogenic potential of adipose tissue derived stromal cells correlates with an altered TGFbeta receptor and BMP profile and is overcome by BMP-6. J Cell Physiol 2007;211:682-91.

31. Ballock RT, Heydemann A, Wakefield LM, Flanders KC, Roberts AB, Sporn MB. TGF-beta 1 prevents hypertrophy of epiphyseal chondrocytes: regulation of gene expression for cartilage matrix proteins and metalloproteases. Dev Biol 1993;158:414-29.

32. Indrawattana N, Chen G, Tadokoro M, Shann LH, Ohgushi H, Tateishi T, Tanaka J, Bunyaratvej A. Growth factor combination for chondrogenic induction from human mesenchymal stem cell. Biochem Biophys Res Commun 2004;320:914-9.

33. Sekiya I, Colter DC, Prockop DJ. BMP-6 enhances chondrogenesis in a subpopulation of human marrow stromal cells. Biochem Biophys Res Commun 2001;284:411-8.

34. Sekiya I, Vuoristo JT, Larson BL, Prockop DJ. In vitro cartilage formation by human adult stem cells from bone marrow stroma defines the sequence of cellular and molecular events during chondrogenesis. Proc Natl Acad Sci U S A 2002;99:4397-402.

35. Dallas SL, Rosser JL, Mundy GR, Bonewald LF. Proteolysis of latent transforming growth factor-beta (TGF-beta )-binding protein-1 by osteoclasts. A cellular mechanism for release of TGF-beta from bone matrix. J Biol Chem 2002;277:21352-60.

36. Santra M, Reed CC, Iozzo RV. Decorin binds to a narrow region of the epidermal growth factor (EGF) receptor, partially overlapping but distinct from the EGF-binding epitope. J Biol Chem 2002;277:35671-81.

37. Suzawa M, Takeuchi Y, Fukumoto S, Kato S, Ueno N, Miyazono K, Matsumoto T, Fujita T. Extracellular matrix-associated bone morphogenetic proteins are essential for differentiation of murine osteoblastic cells in vitro. Endocrinology 1999;140:2125-33. 


\section{FIGURE LEGENDS}

Figure 3.1. The morphology and proliferative rate of ASCs grown in plastic flasks (Plastic), AECM-coated flasks (AECM), or SECM-coated flasks (SECM). (A) Representative phase contrast images of passage 2 ASCs; (B) the cell numbers of ASCs expanded for two passages. Data are shown as average \pm SD for $n=4$. ${ }^{*} p<0.05$ compared with ASCs cultured in Plastic at the same passage. ${ }^{*} p<0.05$ compared with ASCs at passage 1. (C) Flow cytometry was used to measure intracellular ROS level in ASCs after expansion in Plastic, AECM, or SECM. (D) The mean fluorescence intensity (MFI) was for the intracellular level of ROS in ASCs. Data are shown as average \pm SD for $n=4 . \$ p<0.05$ compared with ASCs cultured in AECM in the same passage.

Figure 3.2. Histology and immunohistochemistry of ASC-pellets after 14-day chondrogenic induction in a serum-free chondrogenic medium (CM) supplemented with $10 \mathrm{ng} / \mathrm{mL}$ TGF- $\beta 3$ or $10 \mathrm{ng} / \mathrm{mL}$ TGF- $\beta 3$ combined with $10 \mathrm{ng} / \mathrm{mL}$ BMP-6. ASCs from passage 1 in Plastic (P1.Plastic) were expanded for two passages in Plastic (P3.Plastic), AECM (P3.AECM), or SECM (P3.SECM). Safranin O staining (Saf.O) was used to detect sulfated glycosaminoglycans (GAGs), and immunohistochemical staining (IHC) was for type I (COL I) and type II (COL II) collagen. The scale bar is $800 \mu \mathrm{m}$.

Figure 3.3. Biochemical analysis for GAG (A) and DNA content (B) in ASC-pellets after a 14-day chondrogenic induction in a serum-free chondrogenic medium supplemented with 10 $\mathrm{ng} / \mathrm{mL}$ TGF- $\beta 3$ (T) or $10 \mathrm{ng} / \mathrm{mL}$ TGF- $\beta 3$ combined with $10 \mathrm{ng} / \mathrm{mL}$ BMP-6 (T+B). ASCs from passage 1 in Plastic (P1.Plastic) were expanded for two passages in Plastic (P3.Plastic), AECM (P3.AECM), or SECM (P3.SECM). The rate of GAG to DNA (C) was used to represent chondrogenic index. Data are shown as average \pm SD for $n=5 .{ }^{*} p<0.05$ compared with ASC-pellets incubated in a medium with TGF- $\beta 3$ alone. ${ }^{*} p<0.05$ compared with ASC-pellets from P3.Plastic incubated in the same medium. ${ }^{\$} p<0.05$ compared in the pellets from expanded ASCs in AECM or SECM in the same medium. 
Figure 3.4. TaqMan real-time PCR for the evaluation of chondrogenic markers (Col I, Col II, aggrecan, and Sox9) at mRNA levels in ASC-pellets after a 14-day chondrogenic induction in a serum-free chondrogenic medium supplemented with $10 \mathrm{ng} / \mathrm{mL}$ TGF- $\beta 3$ (T) or $10 \mathrm{ng} / \mathrm{mL}$ TGF-33 combined with $10 \mathrm{ng} / \mathrm{mL}$ BMP-6 (T+B). ASCs from passage 1 in Plastic (P1.Plastic) were expanded for two passages in Plastic (P3.Plastic), AECM (P3.AECM), or SECM (P3.SECM). 18S rRNA was used as an internal control. * $p<0.05$ compared with ASC-pellets incubated in a medium with TGF- $\beta 3$ alone at the same time point. ${ }^{*} p<0.05$ compared with ASC-pellets incubated in the same medium. ${ }^{\$} p<0.05$ compared in the pellets from expanded ASCs in AECM or SECM in the same medium.

Figure 3.5. TaqMan real-time PCR for the evaluation of hypertrophic markers (Col $X$ and MMP-13) at mRNA levels in ASC-pellets after 14-day chondrogenic induction in a serum-free chondrogenic medium supplemented with $10 \mathrm{ng} / \mathrm{mL}$ TGF- $\beta 3$ (T) or $10 \mathrm{ng} / \mathrm{mL}$ TGF- $\beta 3$ combined with $10 \mathrm{ng} / \mathrm{mL}$ BMP-6 (T+B). ASCs from passage 1 in Plastic (P1.Plastic) were expanded for two passages in Plastic (P3.Plastic), AECM (P3.AECM), or SECM (P3.SECM). 18S rRNA was used as an internal control. * $p<0.05$ compared with ASC-pellets incubated in a medium with TGF- $\beta 3$ alone. ${ }^{*} p<0.05$ compared with ASC-pellets incubated in the same medium. ${ }^{\$} P<0.05$ compared in the pellets from expanded ASCs in AECM or SECM in the same medium.

Figure 3.6. Adipogenic differentiation of ASCs was evaluated after expansion in Plastic, AECM, or SECM for two passages and incubation in adipogenic medium for 14 days. (A) Oil Red O staining for oil drops produced by adipocytes with hematoxylin as a counterstain. (B) TaqMan real-time PCR for the evaluation of adipogenic genes (LPL, PPARY and C/EBPa). 18S RNA was used as an internal control. Lower case letters (i.e. a, b, and c) indicate the results of one-way analysis of variance (ANOVA) at $p<0.05$. Groups with a letter in common are not statistically different from one another. Error bars represent the mean \pm SD for $n=4$. 
Figure 3.1

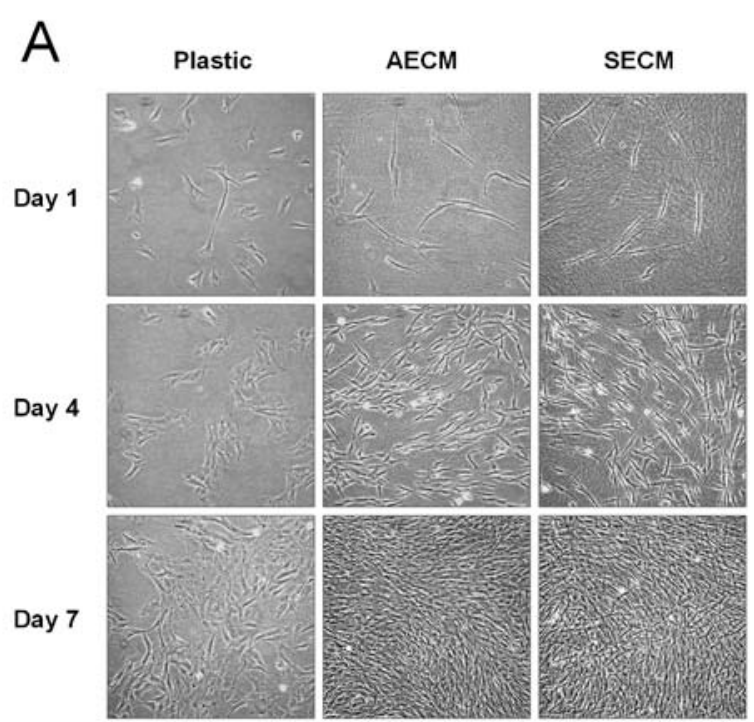

C
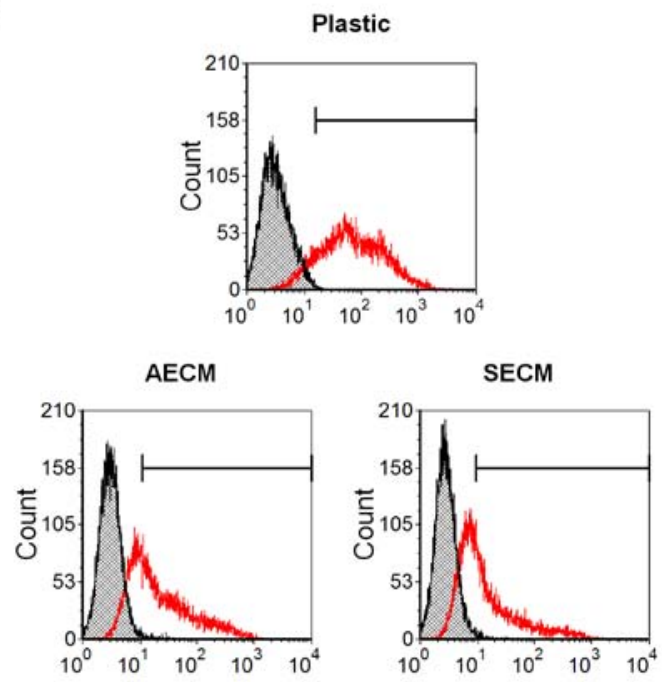

B
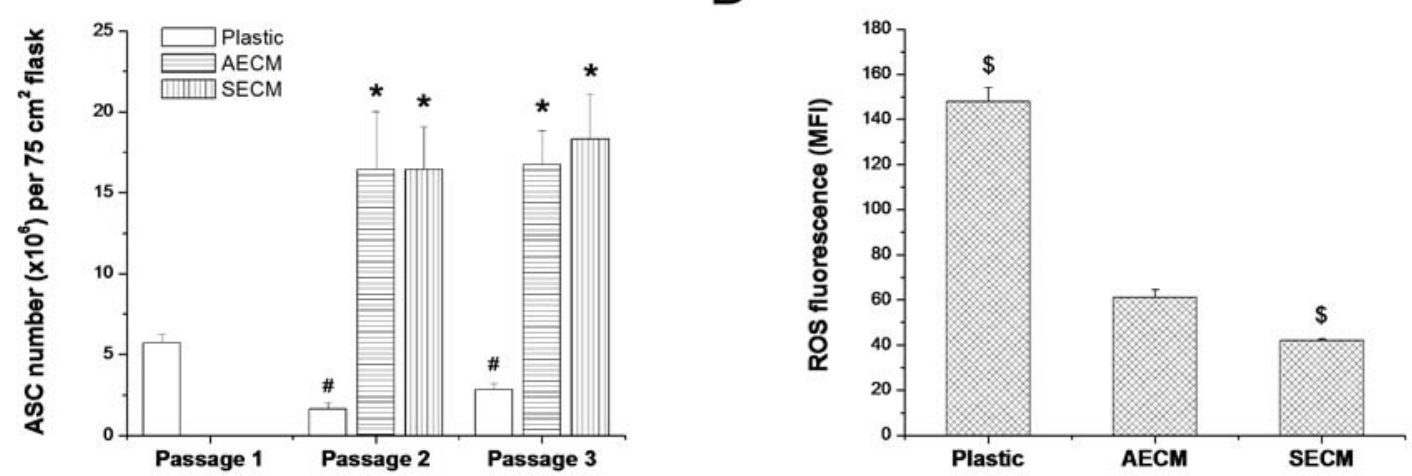
Figure 3.2

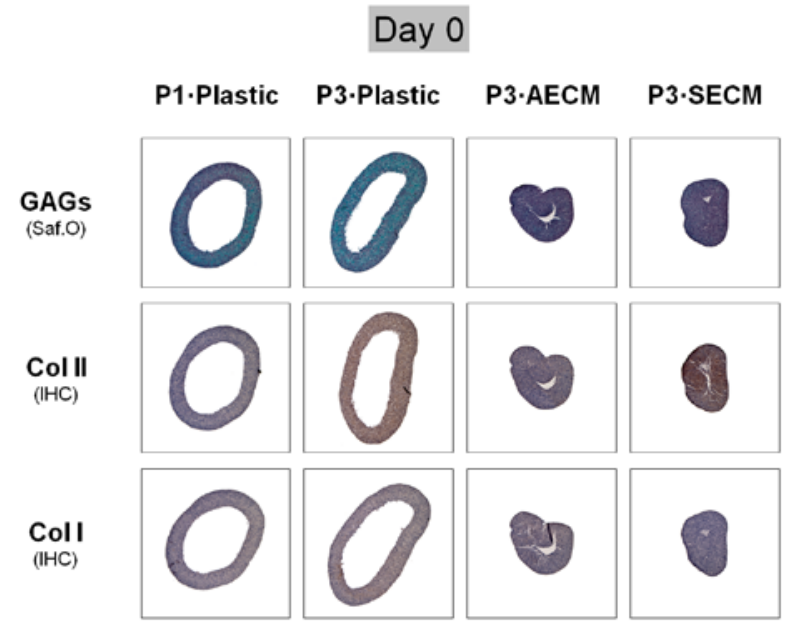

$\mathrm{CM}$ with $10 \mathrm{ng} / \mathrm{mL}$ TGF- $\beta 3$

$\mathrm{CM}$ with $10 \mathrm{ng} / \mathrm{mL}$ TGF- $\beta 3$ and BMP-6

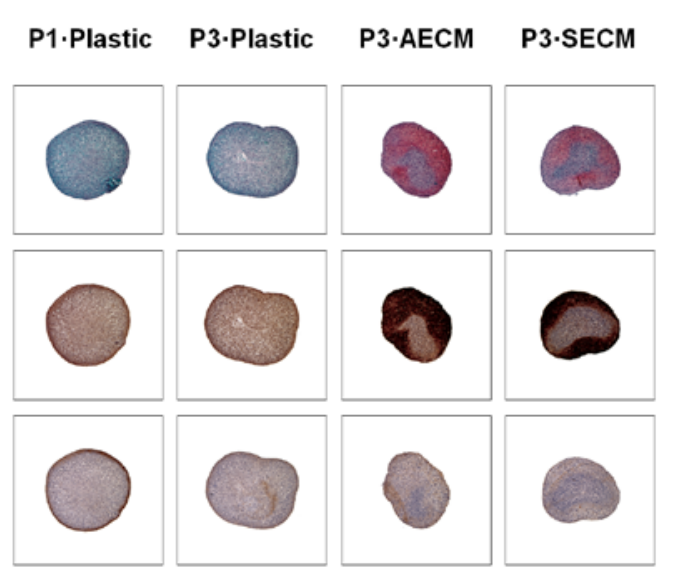

Day 7

\begin{tabular}{lll} 
P1.Plastic P3.Plastic P3.AECM P3.SECM & \\
\hline
\end{tabular}

GAGs
(Saf.O)
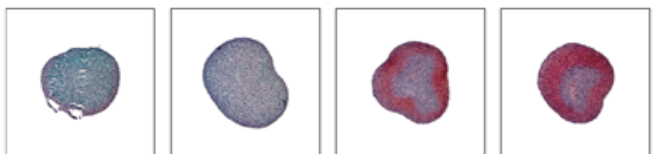

Col II
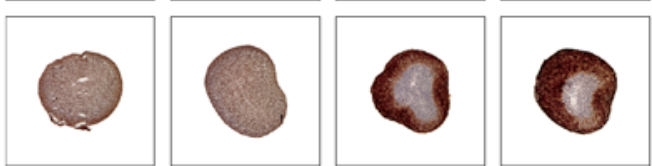

$\underset{(\mathrm{IHC})}{\text { Coll }}$
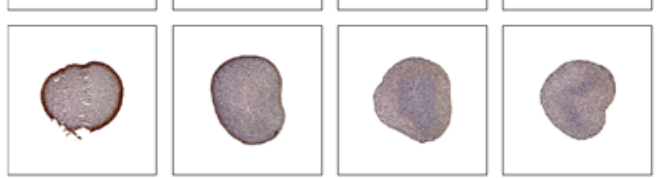

Day 14
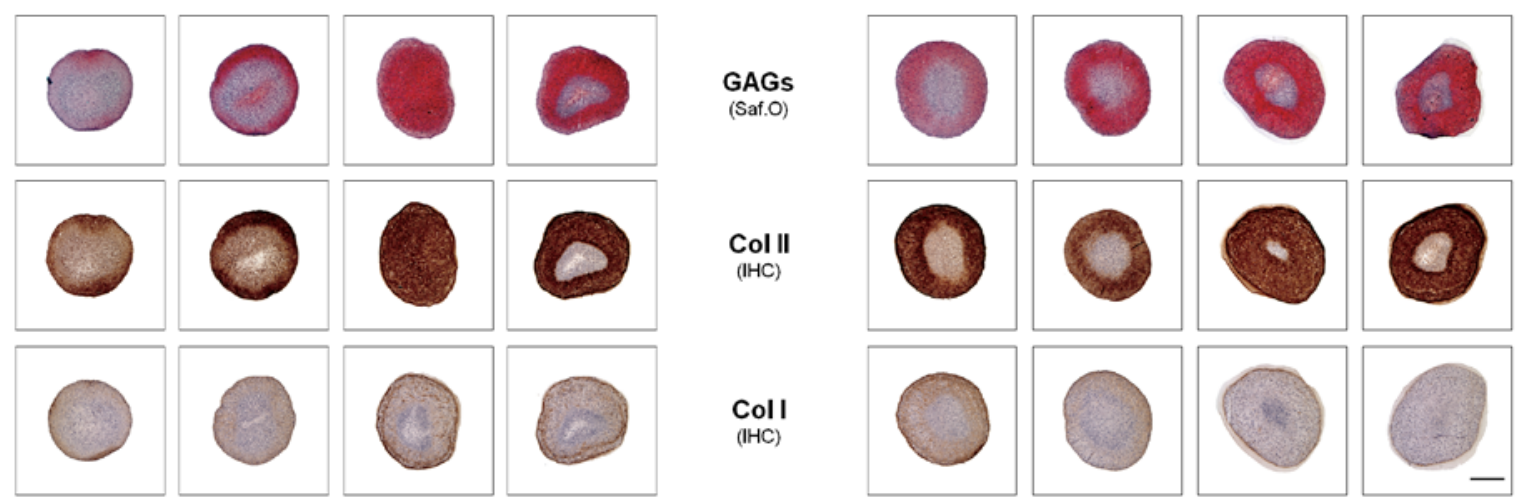
Figure 3.3
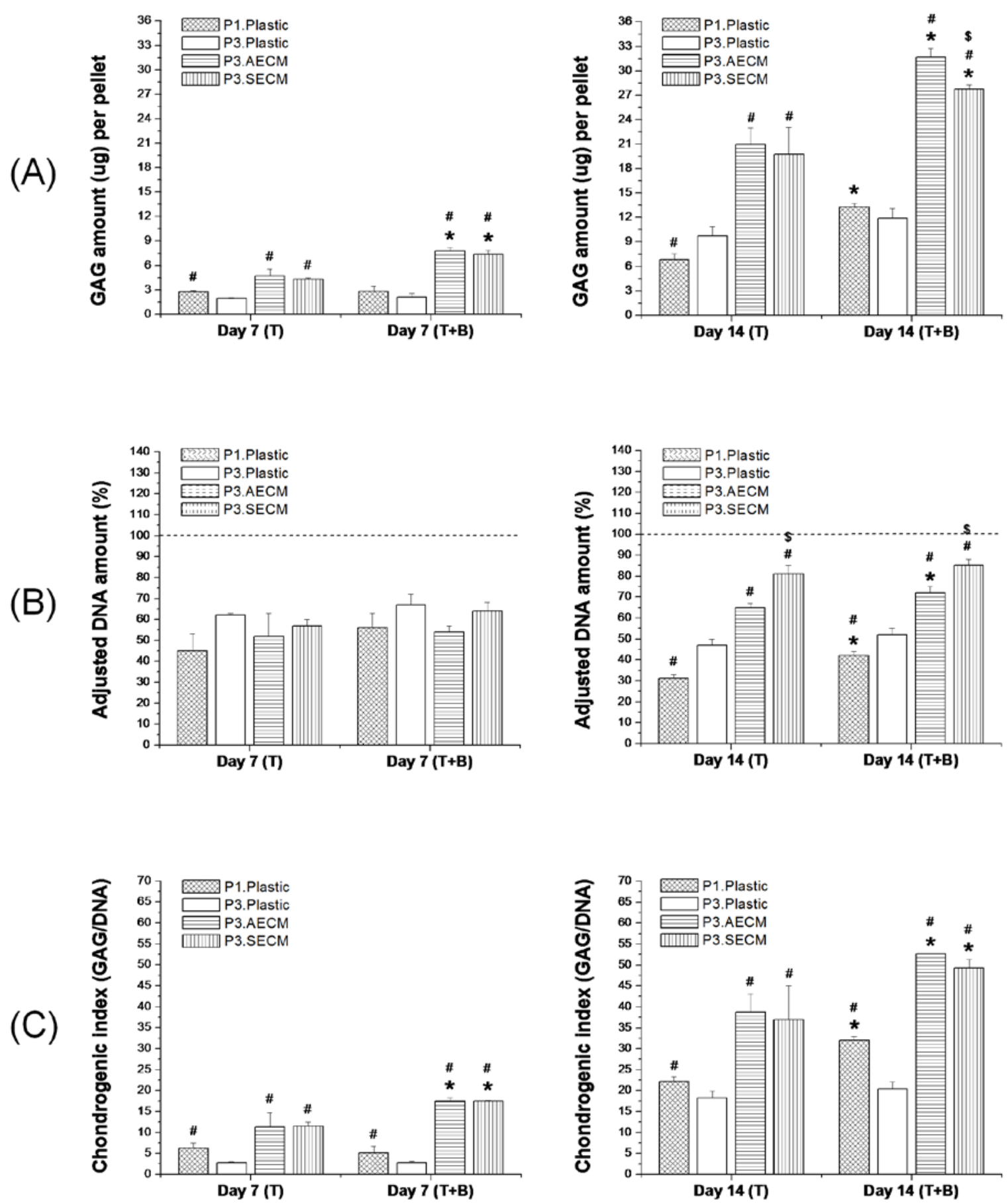
Figure 3.4

(A)
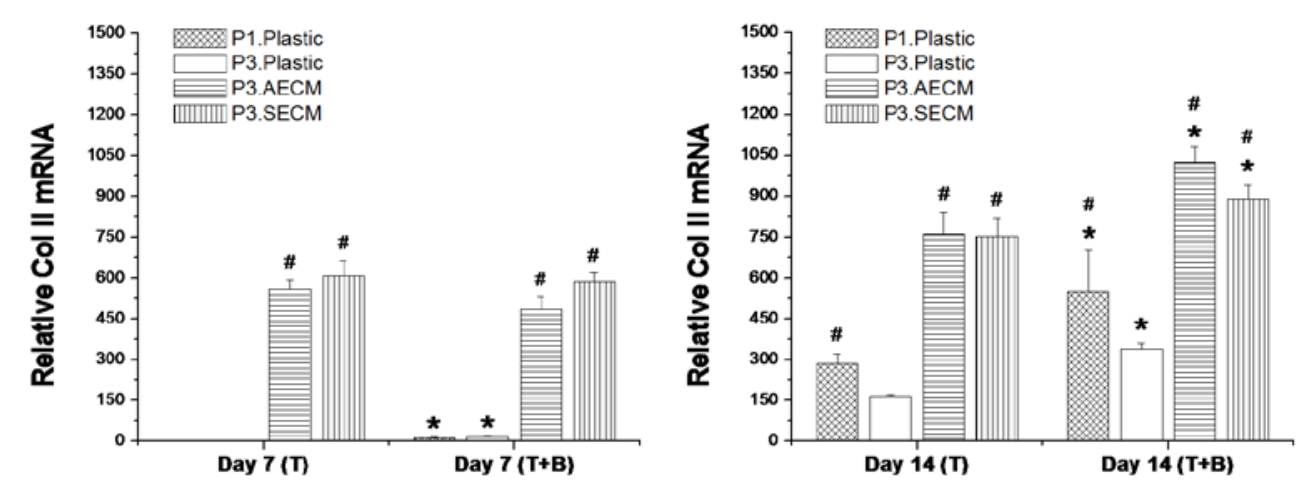

(B)
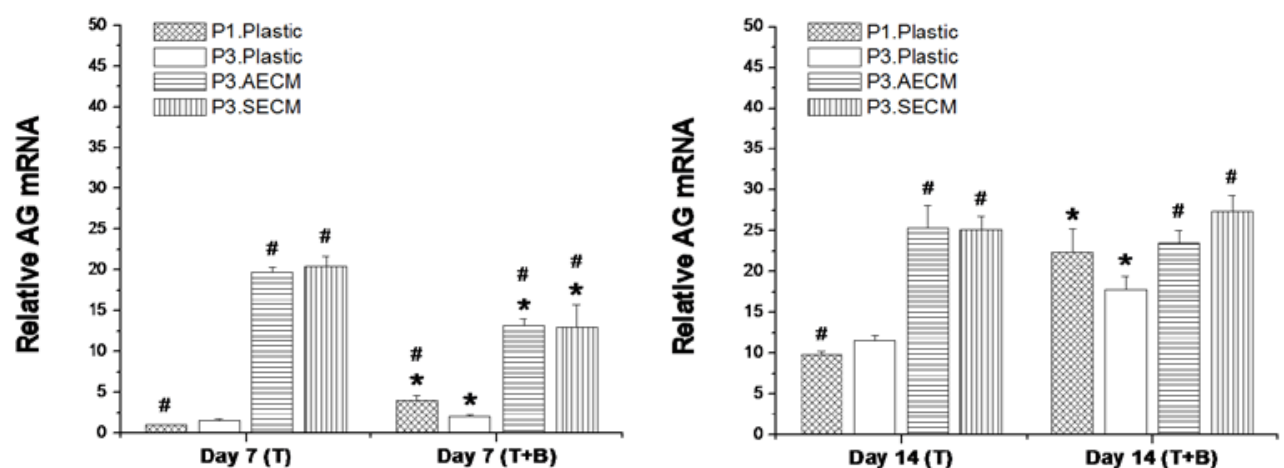

(C)
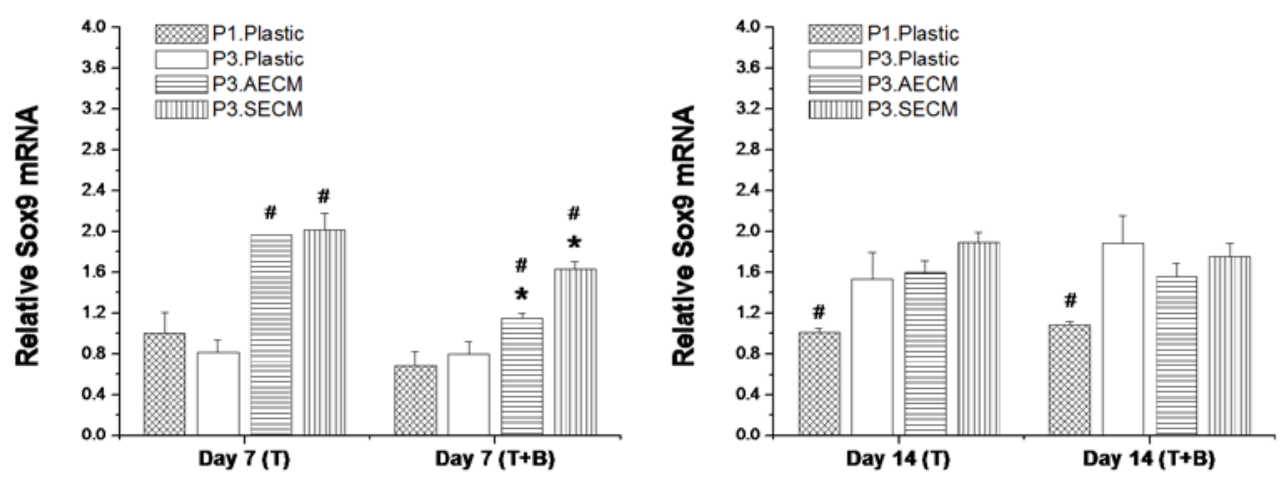

(D)
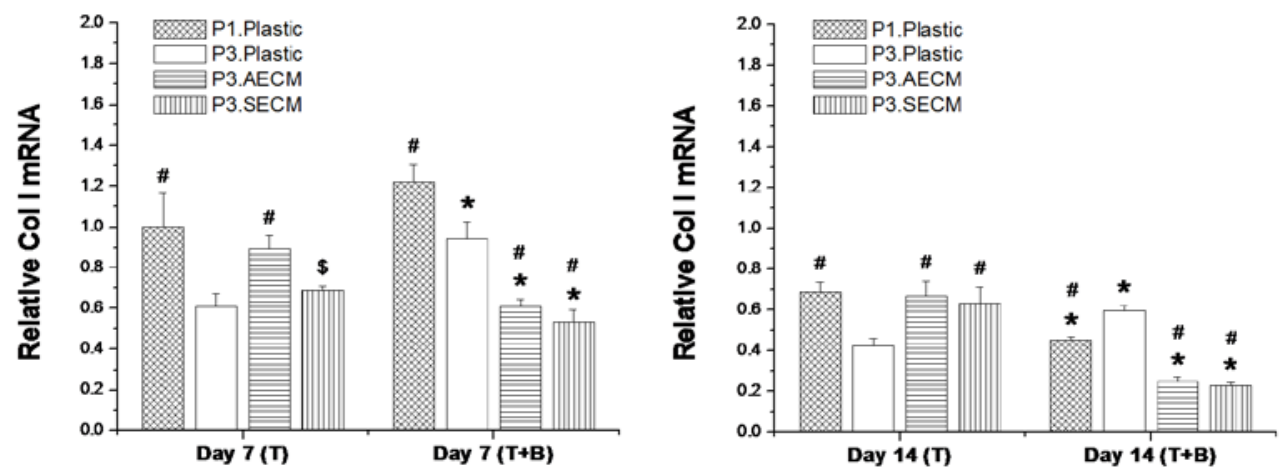
Figure 3.5
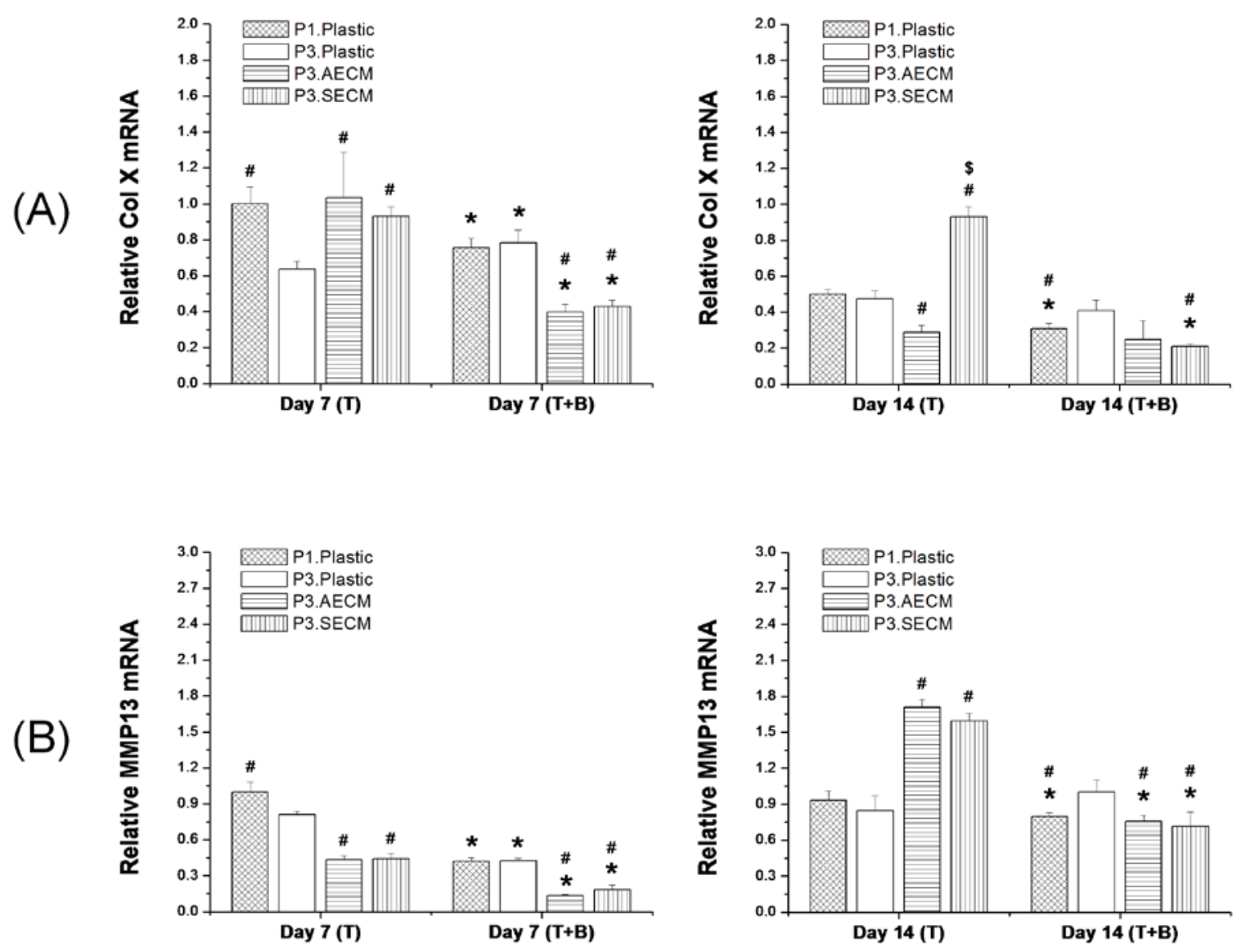
Figure 3.6

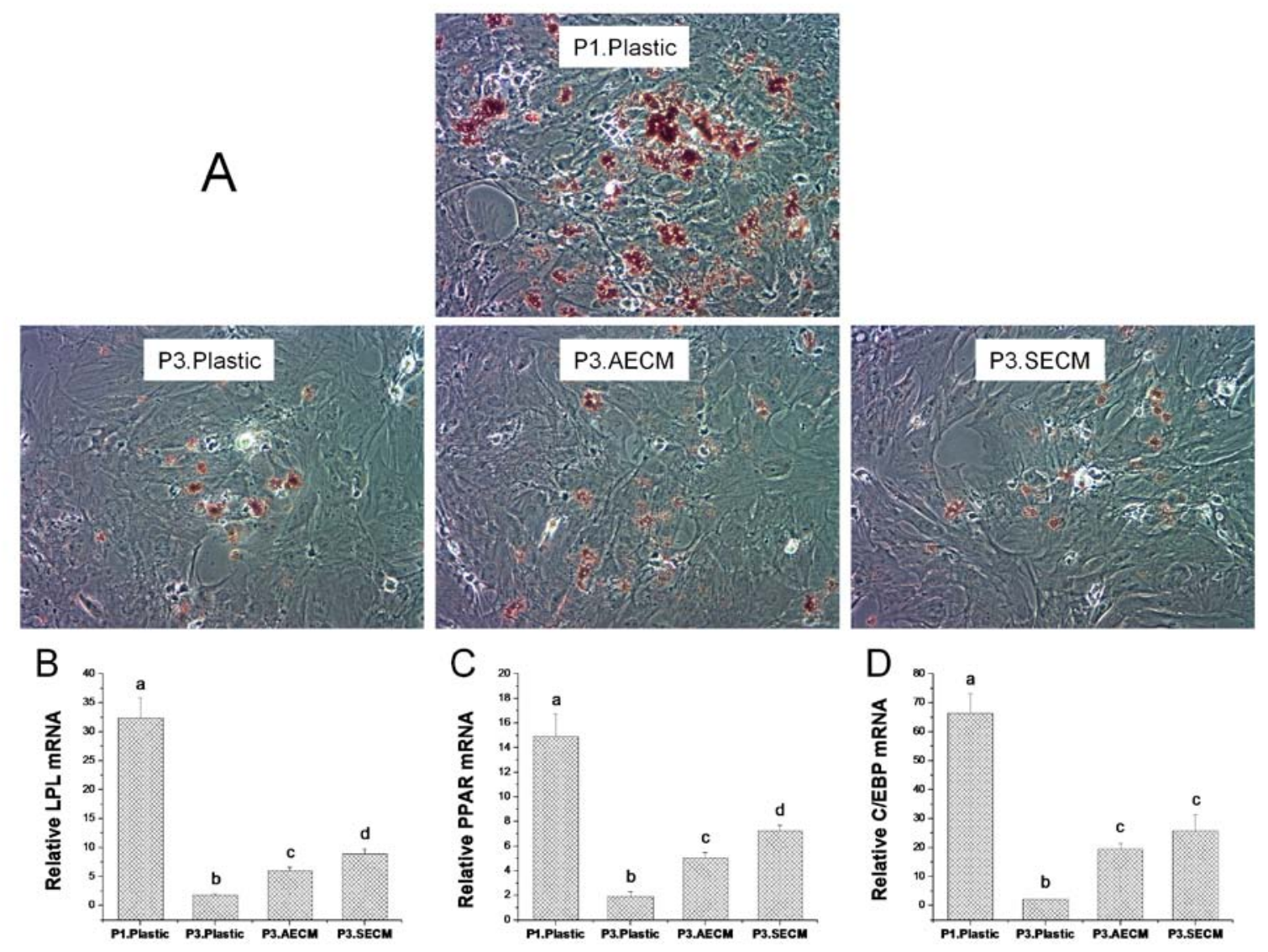




\title{
Chapter 4
}

\section{Extracellular Matrix Deposited by Synovium-Derived Stem Cells Delays Chondrocyte Dedifferentiation and Enhances Redifferentiation}

-- A novel ex vivo expansion system for autologous chondrocyte - based cartilage repair

\author{
Fan He, B.S., and Ming Pei, M.D., Ph.D.
}

Stem Cell and Tissue Engineering Laboratory, Department of Orthopaedics and Division of Exercise Physiology, West Virginia University, Morgantown, West Virginia 26506

Key words: Extracellular matrix, Cartilage regeneration, Synovium-derived stem cell, Chondrocyte, Dedifferentiation, Redifferentiation

Running title: ECM - Based 3D Chondrocyte Expansion System 


\begin{abstract}
Articular cartilage has a limited capacity for self heal once damaged. Autologous chondrocyte transplantation is a promising approach for cartilage defect repair. However, in vitro expansion can cause chondrocyte dedifferentiation. Our aim is to assess the rejuvenation effect of extracellular matrix (ECM) deposited by synovium-derived stem cells (SDSCs) on articular chondrocyte expansion and maintenance of differentiation status and redifferentiation capacity. Passage 0 (P0) porcine articular chondrocytes were expanded for six passages on plastic flasks (Plastic), SDSC-derived ECM (ECM), or substrate switching from either Plastic to ECM (PtoE) or ECM to Plastic (EtoP). Cell morphology, gene expression profiles and immunophenotypes at each passage were used to characterize differentiation status of expanded cells. Expanded chondrocytes at P0, P2 and P6 were assessed for redifferentiation capacity in a pellet culture system treated with either TGF- $\beta 1$ or serum-containing medium for 14 days, using histology, immunohistochemistry, biochemistry, western blot and real time PCR. We found that ECM not only greatly enhanced chondrocyte expansion but also delayed dedifferentiation of expanded chondrocytes. Intriguingly, compared to a dramatic decrease in CD90+/CD105+ cells and CD90+ cells, CD105+ cells dramatically increased when chondrocytes were plated on Plastic; on the contrary, ECM expansion dramatically increased CD90+ cells and delayed the decrease of CD90+/CD105+ cells. Interestingly, expanded chondrocytes on ECM also acquired a strong redifferentiation capacity, particularly in the pellets treated with TGF- $\beta 1$. In conclusion, the ratio of CD90 to CD105 may serve as a marker indicative of proliferation and redifferentiation capacity of dedifferentiated chondrocytes. ECM deposited by SDSCs provides a tissue-specific 3D microenvironment for ex vivo expansion of articular chondrocytes while retaining redifferentiation capacity, suggesting that ECM may provide a novel approach for autologous chondrocyte - based cartilage repair.
\end{abstract}




\section{INTRODUCTION}

Articular cartilage is a unique, hypocellular and avascular tissue, mostly made of extracellular collagens and proteoglycans (PGs); it has a limited ability to self heal after trauma and degenerative disease. ${ }^{1}$ Since the last century, various clinical and research approaches have been adopted to repair articular cartilage damage, including arthroscopic abrasion, transplantation of chondrocytes, periosteum graft, mesenchymal stem cells (MSCs), or tissue constructs derived from MSCs. Each method, however, has disadvantages in achieving satisfactory effects in patients or animal studies. Autologous chondrocyte transplantation (ACT), the most current and clinically accepted method for cartilage repair, depends on the introduction of autologous chondrocytes into the defect area. ${ }^{2,3}$ The big hurdle for this technique is the limited expandability and dedifferentiation of chondrocytes characterized by losing round morphology and changing to a spindle shape. Meanwhile, collagen II and aggrecan dramatically decrease in contrast to the increase in collagen $\mathrm{I}^{4,5}$ Dedifferentiated chondrocytes are able to redifferentiate to express cartilage-specific genes and proteins in a three-dimensional (3D) culture system, such as pellet, ${ }^{6}$ agarose gel, ${ }^{4}$ alginate beads, ${ }^{7}$ or hybrid scaffold ${ }^{8}$; however, late-passage chondrocytes tend to lose their capacity to generate cartilage tissue. $^{9}$

The prevention of dedifferentiation or the preservation of chondrocyte quality during in vitro expansion is one of the major tasks necessary for the innovation of regenerative cartilage medicine. Traditional cell culture on two-dimensional (2D) substrate lacks a proper microenvironment and is proposed to be responsible for the dedifferentiation of chondrocytes; this problem can be overcome by using in vitro 3D models because of its ability to mimic the in vivo environment. ${ }^{10}$ There is increasing evidence demonstrating that superficial zone protein (SZP) synthesized by both chondrocytes and synovial cells bordering the joint cavity ${ }^{11}$ provides a protective microenvironment for cartilage progenitor cells at the surface of articular cartilage. ${ }^{12}$ Our most recent study developed a novel 3D expansion system based on the extracellular matrix (ECM) deposited by synovium-derived stem cells (SDSCs), which dramatically enhanced SDSC proliferation and chondrogenic differentiation potential. ${ }^{13}$ In this 
study, SDSCs serve as a tissue-specific stem cell for chondrogenesis; ${ }^{14}$ we hypothesized that SDSC-derived ECM could provide a tissue-specific microenvironment by improving articular chondrocyte proliferation, delaying dedifferentiation, and enhancing redifferentiation potential. 


\section{MATERIALS AND METHODS}

Isolation of porcine articular chondrocytes and SDSCS

Two 3-month-old pigs were collected from a local slaughterhouse and harvested to provide articular cartilage and synovial tissue from the knee joints. The minced cartilage was digested in $0.2 \%$ collagenase $P$ (Roche, Indianapolis, $I N$ ) at $37^{\circ} \mathrm{C}$ overnight. The finely minced synovial tissue was digested in $0.1 \%$ trypsin (Roche) and then placed in $0.1 \%$ collagenase $\mathrm{P}$ at $37^{\circ} \mathrm{C}$ for $2 \mathrm{~h}$. After filtration through $70-\mu \mathrm{m}$ nylon filter, both cells were plated in culture medium [DMEM containing 10\% fetal bovine serum (FBS), $100 \mathrm{U} / \mathrm{mL}$ penicillin, $100 \mu \mathrm{g} / \mathrm{mL}$ streptomycin, and $0.25 \mu \mathrm{g} / \mathrm{mL}$ fungizone (Invitrogen, Carlsbad, CA)]. Synovial fibroblasts were negatively isolated from primary culture and characterized as SDSCs according to our previous study. ${ }^{14}$

\section{Preparation of SDSC-derived ECM}

The preparation of SDSC-derived ECM was described in our previous study (13). Briefly, cell culture flasks were pre-coated with fibronectin (BD Biosciences, Bedford, MA) for $1 \mathrm{~h}$ at $37^{\circ} \mathrm{C}$. When the plated SDSCs reached 90\% confluence, $50 \mu \mathrm{M}$ L-ascorbic acid phosphate (Wako, Richmond, VA) was added for eight days. After washing with phosphate buffered saline (PBS), ECM was incubated in $0.5 \%$ Triton X-100 containing $20 \mathrm{mM}$ ammonium hydroxide for 5 min followed by100 units/mL DNase (Sigma-Aldrich, St.Louis, MO) for $60 \mathrm{~min}$.

\section{Ex vivo expansion of chondrocytes}

Passage 0 (P0) chondrocytes were expanded for six passages on two substrates: plastic flasks ("Plastic") and SDSC-derived ECM ("ECM"). To identify if the substrate switch would affect cell expansion and redifferentiation capacity (short-term effect), P4 chondrocytes initially expanded on Plastic were plated on ECM for two more passages ("PtoE") or from ECM to Plastic for two more passages ("EtoP"). Chondrocyte morphology expanded on Plastic or ECM was assessed using phase contrast microscope and immunoflurescent microscope. Briefly, P4 chondrocytes were stained with Vybrant Dil cell-labeling solution (Invitrogen) and plated on Plastic or ECM for three days. The samples were fixed in $4 \%$ 
paraformaldehyde for 15 min and blocked in 1\% BSA (Sigma) for $30 \mathrm{~min}$, followed by one-hour-incubation in primary antibody against collagen I (Abcam, Cambridge, MA) and then secondary antibody of Alexa Fluor ${ }^{\circledR} 488$ donkey anti-mouse IgG (H+L) (Invitrogen). The samples were counterstained by mounting in Slow Fade ${ }^{\circledR}$ Gold anti-fade reagent with DAPI (Invitrogen). Immunofluorescent images were obtained with a Nikon Eclipse E800 microscope (Japan) and processed by SPOT software (Diagnostic Instruments Inc., Sterling Heights, MI).

\section{Flow cytometry}

The expanded chondrocytes from each passage were analyzed using the following primary antibodies: CD14 (Abcam), CD90 (BD Biosciences Pharmingen, San Diego, CA), and CD105 (GeneTex Inc., San Antonio, TX). IgG1 and IgG2a (Beckman Coulter, Fullerton, CA) were used as the isotype controls, and R-Phycoerythrin Conjugated Goad Anti-Mouse IgG $(\mathrm{H}+\mathrm{L})$ was used as the secondary antibody. Samples $(n=3)$ of each $0.2 \times 10^{6}$ chondrocytes were incubated in cold PBS containing 0.1\% ChromPure Swine IgG (Jackson ImmunoResearch Laboratories, West Grove, PA) and $1 \% \mathrm{NaN}_{3}$ for $30 \mathrm{~min}$. Then chondrocytes were incubated in the primary antibodies followed by the secondary antibody for $30 \mathrm{~min}$ in the dark. The labeled chondrocytes were analyzed on a BD dual laser FACS Calibur (BD Biosciences) using FCS Express software package (De Novo Software, Los Angeles, CA).

\section{Redifferentiation of expanded chondrocytes in a pellet culture system}

The expanded chondrocytes $\left(3 \times 10^{5}\right)$ from P0, P2 and P6 were centrifuged in a 15-mL polypropylene tube at $500 \mathrm{~g}$ for $5 \mathrm{~min}$. After 24-hour-incubation (day 0), the pellets were incubated in two culture mediums: one was serum-free chondrogenic medium [high-glucose DMEM, $40 \mu \mathrm{g} / \mathrm{mL}$ proline (Sigma), $100 \mathrm{nM}$ dexamethasone (Sigma), $100 \mathrm{U} / \mathrm{mL}$ penicillin, 100 $\mu \mathrm{g} / \mathrm{mL}$ streptomycin, $0.1 \mathrm{mM}$ ascorbic acid-2-phosphate, and ITS ${ }^{\mathrm{TM}}$ Premix (BD Biosciences) with the supplementation of $10 \mathrm{ng} / \mathrm{mL}$ of transforming growth factor beta1 (TGF- $\beta 1, R \& D$ Systems, Minneapolis, MN)] (14) ("TGF- $\beta 1$-containing medium") and the other culture medium was regular medium for chondrocyte-based tissue construct culture [high-glucose DMEM, 10\% FBS, $100 \mathrm{U} / \mathrm{mL}$ penicillin, $100 \mu \mathrm{g} / \mathrm{mL}$ streptomycin, $10 \mathrm{mM}$ HEPES (Sigma), 0.1 
$\mathrm{mM}$ non-essential amino acids (Sigma), $0.4 \mathrm{mM}$ proline, and $50 \mathrm{mg} / \mathrm{L}$ L-ascorbic acid] (8) ("serum-containing medium"). The pellets were incubated at $5 \% \mathrm{CO}_{2}, 5 \% \mathrm{O}_{2}, 37^{\circ} \mathrm{C}$ for 14 days.

\section{Histology and Immunohistochemistry}

The chondrocyte pellets $(n=2)$ were fixed in $4 \%$ paraformaldehyde at $4^{\circ} \mathrm{C}$ overnight, followed by dehydration with a gradient ethanol series, clearing with xylene, and embedding in paraffin blocks. Sections $5 \mu \mathrm{m}$ thick were stained with Alcian blue to detect sulfated glycosaminoglycans (GAGs) with fast red as a counterstain. For immunohistochemical analysis, the sections were immunolabeled with primary antibodies against collagen I (Sigma), collagen II (II-II6B3, DSHB, lowa City, IA), and collagen X (Sigma), respectively. A secondary antibody of biotinylated horse anti-mouse IgG (Vector, Burlingame, CA) was incubated on the sections for 30 min. Immunoactivity was detected using Vectastain $A B C$ reagent (Vector) with 3,3'-diaminobenzidine (DAB) as a substrate. Hematoxylin (Vector) served as a counterstain.

\section{Biochemical analysis for DNA and GAG content}

For biochemical analysis, pellets $(n=5)$ were digested at $60^{\circ} \mathrm{C}$ for $6 \mathrm{~h}$ with $125 \mu \mathrm{g} / \mathrm{mL}$ papain in PBE buffer (100 mM phosphate, $10 \mathrm{mM}$ EDTA, pH 6.5) containing $10 \mathrm{mmol} / \mathrm{L}$ cysteine. The amount of DNA in each pellet was measured using the Quanti ${ }^{\mathrm{TM}}$ PicoGreen $^{\circledR}$ dsDNA assay kit (Invitrogen) with a CytoFluor ${ }^{\circledR}$ Series 4000 (Applied Biosystems, Foster City, CA). Sulfated GAGs were measured using $16 \mu \mathrm{g} / \mathrm{mL}$ of dimethylmethylene blue (DMMB) dye and a Spectronic $^{\mathrm{TM}}$ BioMate $^{\mathrm{TM}} 3$ Spectrophotometer (Thermo Scientific, Milford, MA) with bovine chondroitin sulfate (Sigma) as a standard.

\section{TaqMan $^{\circledR}$ quantitative real-time PCR}

Total mRNA from pellets $(n=5)$ was extracted using TRIzol $^{\circledR}$ (Invitrogen) and $1 \mu \mathrm{g}$ of mRNA was converted to cDNA with High-Capacity cDNA Archive Kit as recommended by the manufacturer (Applied Biosystems). Chondrogenic (aggrecan, collagen I and II) and hypertrophy [collagen X, matrix metalloproteinase 13 (MMP13), and alkaline phosphate 
(ALP)] marker genes were customized by Applied Biosystems as part of the Custom $\operatorname{TaqMan}^{\circledR}$ Gene Expression Assays (Table 1). Eukaryotic $18 \mathrm{~S}$ RNA (Assay ID: HS99999901_S1 ABI) was carried out as the endogenous control gene. Real-time PCR was performed with the iCycler $\mathrm{iQ}^{\mathrm{TM}}$ Multi Color RT-PCR Detection and calculated by computer software (Perkin-Elmer, Waltham, MA).

\section{Western blot}

To assess non-dissolvable collagen II expression in extracellular matrix, SDSC pellets $(n=3)$ were lyophilized and incubated in $4 \mathrm{M}$ guanidine hydrochloride ( $\mathrm{GuHCl}$, Sigma) for $20 \mathrm{~h}$ at $4^{\circ} \mathrm{C}$, then digested with $0.6 \mathrm{mg} / \mathrm{ml}$ of pepsin (Sigma) in $0.5 \mathrm{M}$ acetic acid at $4^{\circ} \mathrm{C}$ for $48 \mathrm{~h}$ at a ratio of 10:1 (mg/mg) dry sample to pepsin. The samples were centrifuged at $48,000 \mathrm{~g}$ for $1 \mathrm{~h}$, and the supernatant was lyophilized and dissolved in radioimmunoprecipitation assay (RIPA) buffer with protease inhibitors (Pierce, Rockford, IL). To assess dissolvable collagen II expression in cytoplasm, the pellets were homogenized and dissolved in RIPA buffer with protease inhibitors. Total proteins were quantified using BCA ${ }^{\mathrm{TM}}$ Protein Assay Kit (Pierce). 40 $\mu \mathrm{g}$ of protein from each sample was denatured and separated using NuPAGE ${ }^{\circledR}$ Novex $^{\circledR}$ Bis-Tris Mini Gels (Invitrogen) in the XCell SureLock ${ }^{\mathrm{TM}}$ Mini-Cell (Invitrogen) at 120 volts for 3 $\mathrm{h}$ at $4^{\circ} \mathrm{C}$. Bands were transferred onto a nitrocellulose membrane (Invitrogen) using an XCell $\mathrm{II}^{\mathrm{TM}}$ Blot module (Invitrogen) at 15 volts overnight at $4^{\circ} \mathrm{C}$. The membrane was incubated with a primary monoclonal antibody against collagen II (II-II6B3) in 1\% non-fat milk in TBST for $1 \mathrm{~h}$ at room temperature ( $\beta$-actin served as an internal control), followed by the secondary antibody of HRP-conjugated goat anti-mouse (Pierce) for $40 \mathrm{~min}$ at room temperature and exposure using SuperSignal West Femto Maximum Sensitivity Substrate (Pierce) and CL-XPosure Film (Pierce).

\section{Statistical Analysis}

The Kruskal-Wallis test was used to determine significant differences among all groups and the Mann-Whitney $U$ test was used for pairwise comparison. All statistical analysis was performed with SPSS 13.0 statistical software (SPSS Inc., Chicago, IL). P values less than 0.05 were considered statistically significant. 


\section{RESULTS}

Expansion on ECM acquired a large number of chondrocytes with delayed dedifferentiation To identify the effect of ECM on chondrocyte proliferation and marker gene stability, P0 chondrocytes were expanded on ECM for six passages with Plastic substrate as a control. Compared to the original round morphology, chondrocytes expanded on Plastic exhibited flattened and broad morphology (Figure $4.1 \mathrm{~A}$ and $4.1 \mathrm{~B}_{\mathrm{iii}}$ ); in contrast, ECM-expanded chondrocytes displayed a smaller size with a glistening surface and fibroblast-like shape that were aligned in rows (Figure $4.1 \mathrm{~A}$ and $4.1 \mathrm{~B}_{\mathrm{ii}}$ ). ECM was positively stained with collagen I (Figure 4.1B $\mathrm{B}_{\mathrm{i}}$ ). From P1 to P5, the cell number fold-change in the Plastic group continued to decrease (on average 5-fold); surprisingly, chondrocyte expansion on ECM yielded 4 to 10 times more cells than on Plastic (Figure 4.1C). Despite a sharp decrease of collagen II mRNA in chondrocytes from both groups, ECM expanded chondrocytes restored more collagen II mRNA than those plated on Plastic (Figure 4.1D). In contrast, collagen I mRNA dramatically increased in chondrocytes after isolation from cartilage despite a burst increase at P1 and P4 and a decrease at P6 in the ECM group (Figure 4.1E).

To evaluate the effect of ECM on immunophenotypic changes of articular chondrocytes during in vitro expansion, CD14, CD90 and CD105 were chosen to identify differentiation status of expanded chondrocytes (Figure 4.2A-C and 4.2a-c). After an instant increase at P1, both CD14+ cells and CD90+ cells decreased with passage when chondrocytes were expanded on Plastic; intriguingly, ECM expansion dramatically increased CD14+ cells and CD90+ cells up to P6. On the contrary, compared to an increase in CD105+ cells after expansion on Plastic, ECM expansion exhibited a lower expression of CD105+ cells. The change of culture substrate from either PtoE or EtoP resulted in the same conclusion.

To identify the effect of ECM on CD90+ cells and/or CD105+ cells during in vitro expansion, single positive (CD90+/CD105- or CD105+/CD90-), dual positive (CD90+/CD105+), and dual negative (CD90-/CD105-) cells were measured in expanded chondrocytes at P0, P2, and P6 using flow cytometry (Figure 4.2D). Interestingly, when chondrocytes were expanded on 
Plastic, both CD90+/CD105+ cells and CD90+/CD105- cells decreased dramatically with a sharp increase in CD105+/CD90- cells and CD90-/CD105- cells. However, ECM expansion delayed a decrease in CD90+/CD105+ cells but increased CD90+/CD105- cells despite a concomitant increase in CD90-/CD105- cells.

\section{Chondrocytes expanded on ECM acquired enhanced redifferentiation capacity}

To evaluate the redifferentiation capacity of expanded chondrocytes, pellets from P0, P2 (Plastic and ECM), and P6 (Plastic, PtoE, ECM, and EtoP) were incubated in either TGF- $\beta 1$ or serum-containing medium for 14 days.

Redifferentiation of expanded chondrocytes in a TGF- $\beta 1$-containing medium Histology and immunostaining was performed to evaluate the density and distribution of chondrogenic markers in pellets from expanded chondrocytes. At Day 0 (Supplemental Figure 4.S1), there was no detectable staining for sulfated GAGs or collagen except in the pellets from P0, P2.ECM, and P6.ECM. At day 7 (Supplemental Figure 4.S1) and day 14 (Figure 4.3A), sulfated GAGs and collagen II were distributed intensively throughout the pellets from P0, P2.Plastic, P2.ECM and P6.ECM despite a weaker and comparable staining of collagen I in all pellets except from P0. At P6, the pellet sizes from Plastic and EtoP were smaller than those from ECM and PtoE. There was no detectable collagen $X$ in any pellets (data not shown).

To confirm our histology data, western blot was used to semi-quantify the expression of collagen II at day 7 (Supplemental Figure 4.S2A) and day 14 (Figure 4.3B). We found that collagen II was intensively expressed in both cytoplasm (immature) and extracellular matrix (mature) in the pellets from P0, P2.Plastic, P2.ECM, and P6.ECM but not P6.Plastic. P6 chondrocytes expanded on either PtoE or EtoP yielded pellets with enhanced collagen II expression compared to those from P6.Plastic. Biochemical analysis was used to quantify GAGs in the pellets from expanded chondrocytes at day 0 (Figure 4.3C), day 7 (Supplemental Figure 4.S2B), and day 14 (Figure 4.3C). At day 0, pellets of P0 chondrocytes contained the highest GAGs compared to other groups. After incubation in TGF- $\beta 1$-containing 
medium for 14 days, pellets of P2 chondrocytes expanded on ECM exhibited a comparable GAG amount to those plated on Plastic. In contrast, pellets of P6 chondrocytes expanded on ECM yielded the highest level of GAGs, which was 7.9-fold greater than those from P6.Plastic. Intriguingly, pellets of P6 chondrocytes from EtoP exhibited higher GAGs than those from P6.Plastic but lower than those from P6.PtoE. Meanwhile, compared to a time-dependent increase in cell number of pellets from P0 chondrocytes (" $162 \% \pm 7 \%$ " at Day 7 and "204\% $\pm 4 \%$ " at Day 14), the cell number in the pellets from P6.Plastic dropped to $50 \%$ even after a 7-day incubation; however, the pellets from P6 chondrocytes expanded on ECM maintained the cell number at $124 \%$ after a 14-day incubation. The pellets of P6 chondrocytes from EtoP exhibited an increased expansion rate compared to those of P6.Plastic. Expanded chondrocytes from P2.Plastic and P6.ECM yielded pellets with the highest chondrogenic index whereas the pellets from P6.Plastic had the lowest chondrogenic index after 14-day incubation with TGF- $\beta 1$. The pellets of P6 chondrocytes from EtoP yielded a higher chondrogenic index compared to those from P6.Plastic but was lower than those from PtoE.

To assess the effect of ECM on redifferentiation capacity of expanded chondrocytes at the mRNA level, real-time PCR was used to quantify chondrogenic marker genes (Figure 4.4A collagens I and II, and aggrecan) and hypertrophic marker genes (Figure 4.4B - collagen X, ALP, and MMP13) at day 0 (Figure 4.4), day 7 (Supplemental Figure 4.S2C), and day 14 (Figure 4.4). Our data showed that collagen II and aggrecan presented a similar profile of gene expression without significant variations. Despite the highest expression of both genes in pellets from P0 chondrocytes at day 0, 14-day-incubation with TGF- $\beta 1$ yielded pellets with a higher expression of both genes in pellets from chondrocytes of P0, P2.ECM, and P6.ECM. At P6, pellets from chondrocytes expanded on Plastic exhibited the lowest expression of both genes. As an early marker of chondrogenic differentiation, collagen I mRNA decreased in all pellets when incubated in a TGF- $\beta 1$-containing medium. Despite the highest collagen $X$ in chondrocytes from P2.ECM after 14-day incubation with TGF- $\beta 1$, expanded chondrocytes from P6.ECM had comparable collagen X mRNA to those from P6.Plastic. Another two hypertrophic markers, ALP and MMP13, both displayed a higher expression in chondrocytes 
from P6.Plastic than those from P6.ECM (6.1-fold and 9.0-fold, respectively).

\section{Redifferentiation of expanded chondrocytes in a serum-containing medium}

Although the pellets from PO chondrocytes incubated in a serum-containing medium were strongly stained by Alcian blue for sulfated GAGs and immunostained for collagen II as well as non-detectable staining for collagen I as those treated with TGF- $\beta 1$, the pellets from P2 chondrocytes were smaller in size and weaker in staining for both GAGs and collagen II, especially for the pellets from P2.Plastic. More interestingly, 14-day-incubated pellets (Figure 4.5A) were weaker in chondrogenic marker staining than those from 7-day-incubated pellets (Supplemental Figure 4.S1). At P6, only pellets from ECM maintained their size and were positive for sulfated GAGs, collagens I and II; the pellets from other groups were tiny and less stained.

To confirm our histology data, western blot was used to semi-quantify collagen II in pellets incubated in a serum-containing medium (Figure 4.5B). Only three groups, the pellets from P0, P2.ECM, and P6.ECM, could be extracted to yield sufficient proteins for immunoblotting. The data showed that the pellets from PO chondrocytes exhibited the highest expression of collagen II in both cytoplasm and extracellular matrix. Despite undetectable collagen II in those from P6.ECM, the pellets from P2.ECM exhibited detectable collagen II expression only in extracellular matrix. Our biochemical analysis data (Figure 4.5C) showed that, compared to those treated by TGF- $\beta 1$, the pellets from P0 chondrocytes incubated in a serum-containing medium yielded more GAG amounts and a higher DNA ratio as well as chondrogenic index. However, the pellets from P2 chondrocytes incubated in a serum-containing medium exhibited much less redifferentiation response despite the fact that 14-day-pellets from P2.ECM had higher GAG amounts and maintained higher DNA ratio compared to those from P2.Plastic.

Our mRNA data (Figure 4.6) showed that collagen II and aggrecan presented a similar profile of gene expression without significant variation. Both genes exhibited a positive response to serum incubation despite an initial increase at day 7 and a later decrease at day 14 . The 
pellets from P2.ECM and P6.ECM displayed higher expression levels of both marker genes not only before but also after serum incubation compared to the chondrocytes expanded on Plastic. However, all pellets from expanded chondrocytes incubated in a serum-containing medium displayed much less collagen II and aggrecan gene expression compared to culture in a TGF- $\beta 1$-containing medium except the pellets from P0 chondrocytes. There was no significant difference in collagen I expression in the pellets from expanded chondrocytes, either before or after serum treatment. 


\section{DISCUSSION}

A generally accepted opinion is that passage 3-4 is considered as a threshold for irreversible dedifferentiation of chondrocytes. ${ }^{9,11}$ Although multiple chondrocyte passaging decreases their redifferentiation capacity, ${ }^{15}$ our study suggested that the sequential application of ECM (seeding substrate for cell proliferation) and TGF- $\beta$ (growth factor for differentiation) affected chondrocyte expansion and redifferentiation capacity. In our study, chondrocytes expanded on SDSC-derived ECM showed a robust proliferation capacity in a 3D format. The expanded cells on ECM retained more chondrocyte gene expression profiles and immunophenotypes compared to expansion on Plastic. When incubated in a serum-free TGF- $\beta 1$-containing chondrogenic medium, chondrocytes expanded on ECM exhibited enhanced redifferentiation for up to passage 6 compared to expansion on Plastic that retained their redifferentiation only in the early passages.

We found that signs of dedifferentiation appeared even after one passage on Plastic, evidenced by dramatically decreased collagen II mRNA and increased collagen I mRNA compared with the native cartilage; this outcome is in agreement with previous studies. ${ }^{16,17}$ We also found that expansion on ECM not only increased cell number but also delayed the decrease of a chondrocyte differentiation marker - collagen II. Consistent with a previous study, ${ }^{18}$ our data showed that CD14+ cells decreased when chondrocytes were incubated on Plastic. Since CD14 is normally expressed by articular chondrocytes ${ }^{19}$ instead of by MSCs ${ }^{18}$, expansion on ECM boosted CD14+ cells, indicating that ECM was able to rescue differentiated chondrocytes and prevent dedifferentiation during in vitro expansion. This also can be explained by a recent report that chondrocytes cultured on collagen I-coated substrate showed a tendency of suppressed dedifferentiation. ${ }^{20}$ Our previous study demonstrated that ECM deposited by SDSCs, a tissue-specific stem cell for chondrogenesis, provides a 3D microenvironment composed of collagen I-dominated nano-structure fibers; SDSC expansion on ECM exhibited an enhanced proliferation capacity and chondrogenic potential, ${ }^{13}$ which is in accordance with this study and indicative that ECM expanded 
chondrocytes exhibit an enhanced redifferentiation capacity.

Our data also showed that expansion on Plastic instantly increased CD90+ cells at P1 followed by a gradual decrease while continuously increasing CD105+ cells. On the contrary, expansion on ECM increased CD90+ cells while decreasing CD105+ cells. A recent report showed that during monolayer culture, articular chondrocytes expressed higher levels of CD90 and lower levels of CD105 compared to MSCs. ${ }^{21}$ An interesting phenomenon was that there was a phenotype switch in expanded chondrocytes from CD90+/CD105+ to CD105+/CD90- when plated on Plastic or a gradual decrease in CD90+/CD105+ cells concomitant with an increase in CD90+/CD105- when expanded on ECM. As we know, CD90 and CD105 are MSC markers; ${ }^{22}$ increased CD90+ cells are also associated with cell proliferation capacity and chondrogenic potential ${ }^{23,24}$ as well as the induction of stress fibers in articular chondrocytes ${ }^{19}$. CD105 (also termed endoglin or $\mathrm{SH}_{2}{ }^{25}$ ) was expressed on articular chondrocytes at a high intensity, where endoglin forms a heteromeric complex with betaglycan. ${ }^{26}$ Endoglin-betaglycan association may be critical for achieving a balance between the positive and negative regulation of TGF- $\beta$ signaling. Compared to overexpression of betaglycan enhanced TGF- $\beta$ responses, overexpression of endoglin resulted in an inhibition of TGF- $\beta$ signaling. ${ }^{27}$ Like bone marrow MSCs, dedifferentiated chondrocytes were positive for the CD105 marker. This suggests that, within a chondrocyte preparation, subpopulations with higher capacity to form cartilage might correspond to those with progenitor characteristics. Our pellet culture data suggested that ECM expanded chondrocytes acquired not only a high proliferation capacity but also an enhanced redifferentiation capacity, indicating that the CD90/CD105 ratio may serve as a useful index to show the proliferation and redifferentiation capacity of dedifferentiated chondrocytes.

To stimulate chondrocyte redifferentiation after in vitro expansion, TGF- $\beta 1$ was chosen to facilitate the formation of cartilage-specific extracellular matrix ${ }^{28}$ with serum-containing medium as a control. Culture medium supplemented with serum was proven to effectively induce freshly isolated chondrocytes to differentiate into cartilage tissue constructs in $3 \mathrm{D}$ biodegradable scaffolds. ${ }^{8}$ Under the serum treatment, however, our study showed that 
chondrocytes expanded on Plastic lose their capacity for redifferentiation as early as P2, suggesting that serum could only maintain the redifferentiation potential of early-expanded chondrocytes. The chondrocytes cultured on ECM were able to maintain cell viability and generate cartilage-specific matrix up to $\mathrm{P} 6$, albeit much weaker than those treated by TGF- $\beta 1$-containing medium. A recent study showed that there is the existence of a certain subpopulation of multipotent MSCs or progenitor cells in the cartilage tissue besides terminally differentiated chondrocytes. ${ }^{29}$ In our study, chondrocytes expanded on ECM behaved more like stem cells or progenitors rather than terminally differentiated cells in terms of rapid proliferation rate and high level of CD90 surface marker. When culturing on ECM, stem cells or progenitors in mixed chondrocyte populations would accelerate the expansion rate compared with mature chondrocytes and those cells have strong chondrogenic potential, evidenced by generating cartilage-specific matrix in response to stimulation by TGF- $\beta 1$ in a pellet culture system. A potential mechanism underlying ECM enhancing chondrocyte proliferation and redifferentiation capacity may be explained by ECM acting through integrin-mediated extracellular signal-activated kinase (ERK) signal transduction pathway. Chondrocyte expansion in monolayer culture shows the expression of osteoblast cadherin (Ob-cadherin) and abundant cell-to-cell contacts, indicative of dedifferentiation. ${ }^{30}$ On the contrary, the 3D expanded cells exhibit lower expression of cadherins than did monolayer expanded cells, especially that of Ob-cadherin, which was inhibited to the level of native chondrocytes. $^{31}$ Silencing of Ob-cadherin expression in monolayer cells induced redifferentiation in dedifferentiated cells, implying that the 3D microenvironment for cell expansion diminishes cell-to-cell contacts like native cartilage, which may result in resistance to dedifferentiation. ${ }^{31}$ ECM would interact with chondrocytes through integrins and other signaling receptors, in which specifically $\alpha_{5} \beta_{1}$ and $\alpha_{v} \beta_{3}$ integrins were proven highly expressed in the cells attaching on the $3 \mathrm{D} \mathrm{ECM}{ }^{32,33}$ Afterwards the phosphorylation of ERK1/2 is upregulated by the activated $\beta_{1}$ integrin via Src/Ras/Raf signaling pathway; ${ }^{34}$ then the high level of phosphorylated ERK1/2 would enhance TGF- $\beta 1$-mediated redifferentiation of expanded chondrocytes on ECM. ${ }^{35,36}$

Our study also investigated the short-term effect of culture substrate on chondrocyte 
expansion and redifferentiation capacity by switching either from Plastic to ECM (PtoE) or from ECM to Plastic (EtoP). Our data suggested that expanded chondrocytes from either PtoE or EtoP acquired higher proliferation and redifferentiation capacity compared to those plated on Plastic but lower than those seeded on ECM. Chondrocytes expanded on PtoE acquired a higher redifferentiation capacity than those on EtoP, suggesting that once the chondrocytes adapt to the ECM environment, the transition from EtoP would deprive the chondrocytes of their expandability and redifferentiation potential. Our study indicated that dedifferentiated or aged chondrocytes would regain their redifferentiation capacity with the help of ECM deposited by SDSCs. ECM provides an in vitro 3D expansion system that may play an important role in autologous chondrocyte transplantation-based cartilage repair.

In summary, we found that ECM deposited by a tissue-specific stem cell was able to efficiently expand primary chondrocytes; ECM-expanded chondrocytes retained their significant redifferentiation capacity. These results demonstrated that SDSC-derived ECM can function as a new ex vivo expansion system to enhance chondrocyte proliferation and redifferentiation potential for future clinical applications. Further studies are needed to investigate whether redifferentiated chondrocytes from expansion on ECM are capable of forming cartilage tissue in vivo, which would ensure the stability and functionality of the acquired chondrocyte phenotype. The mechanisms underlying the interaction between chondrocytes and ECM also need to be further investigated. 


\section{ACKNOWLEDGEMENTS}

The authors thank Suzanne Smith for her help in editing the manuscript and Dr. Kathleen Brundage for her help with the experiments using flow cytometry, which were performed in the West Virginia University Flow Cytometry Core Facility and supported in part by NIH grants RR106440 


\section{REFERENCES}

1. Redman, S.N., Oldfield, S.F., and Archer, C.W. Current strategies for articular cartilage repair. Eur. Cell Mater. 9, 23, 2005.

2. Brittberg, M., Lindahl, A., Nilsson, A., Ohlsson, C., Isaksson, O., and Peterson, L. Treatment of deep cartilage defects in the knee with autologous chondrocyte transplantation. N. Engl. J. Med. 331, 889, 1994.

3. Peterson, L., Minas, T., Brittberg, M., Nilsson, A., Sjogren-Jansson, E., and Lindahl, A. Two- to 9-year outcome after autologous chondrocyte transplantation of the knee. Clin. Orthop. Relat. Res. 374, 212, 2000.

4. Benya, P.D., and Shaffer, J.D. Dedifferentiated chondrocytes reexpress the differentiated collagen phenotype when cultured in agarose gels. Cell 30, 215, 1982.

5. von der, M.K., Gauss, V., von der, M.H., and Muller, P. Relationship between cell shape and type of collagen synthesised as chondrocytes lose their cartilage phenotype in culture. Nature 267, 531, 1977.

6. Pei, M., He, F., Wei, L., and Rawson, A. Melatonin enhances cartilage matrix synthesis by porcine articular chondrocytes. J. Pineal Res. 46, 181, 2009.

7. Hauselmann, H.J., Fernandes, R.J., Mok, S.S., Schmid, T.M., Block, J.A., Aydelotte, M.B., Kuettner K.E., and Thonar, E.J. Phenotypic stability of bovine articular chondrocytes after long-term culture in alginate beads. J. Cell Sci. 107, 17, 1994.

8. Pei, M., Solchaga, L.A., Seidel, J., Zeng, L., Vunjak-Novakovic, G., Caplan, A.I., and Freed, L.E. Bioreactors mediate the effectiveness of tissue engineering scaffolds. FASEB J. 16, 1691, 2002.

9. Dell'Accio, F., De, B.C., and Luyten, F.P. Molecular markers predictive of the capacity of expanded human articular chondrocytes to form stable cartilage in vivo. Arthritis Rheum. 44, 1608, 2001.

10. Yamada, K.M., and Cukierman, E. Modeling tissue morphogenesis and cancer in 3D. Cell 130, 601, 2007.

11. Schumacher, B.L., Hughes, C.E., Kuettner, K.E., Caterson, B., and Aydelotte, M.B. Immunodetection and partial cDNA sequence of the proteoglycan, superficial zone 
protein, synthesized by cells lining synovial joints. J. Orthop. Res. 17, 110, 1999.

12. Dowthwaite, G.P., Bishop, J.C., Redman, S.N., Khan, I.M., Rooney, P., Evans, D.J., Haughton, L., Bayer, S., Thomson, B., Wolfe, M.S., and Archer, C.W. The surface of articular cartilage contains a progenitor cell population. J. Cell Sci. 117, 889, 2004.

13. He, F., Chen, X., and Pei, M. Reconstruction of an in vitro tissue-specific microenvironment to rejuvenate synovium-derived stem cells for cartilage tissue engineering. Tissue Eng. Part A 15, 3809, 2009.

14. Pei, M., He, F., and Vunjak-Novakovic, G. Synovium-derived stem cell-based chondrogenesis. Differentiation 76, 1044, 2008.

15. Mandl, E.W., van der Veen, S.W., Verhaar, J.A., and van Osch, G.J. Multiplication of human chondrocytes with low seeding densities accelerates cell yield without losing redifferentiation capacity. Tissue Eng. 10, 109, 2004.

16. Cournil-Henrionnet, C., Huselstein, C., Wang, Y., Galois, L., Mainard, D., Decot, V., Netter, P., Stoltz, J.F., Muller, S., Gillet, P., and Watrin-Pinzano, A. Phenotypic analysis of cell surface markers and gene expression of human mesenchymal stem cells and chondrocytes during monolayer expansion. Biorheology 45, 513, 2008.

17. Lin, L., Zhou, C., Wei, X., Hou, Y., Zhao, L., Fu, X., Zhang, J., and Yu, C. Articular cartilage repair using dedifferentiated articular chondrocytes and bone morphogenetic protein 4 in a rabbit model of articular cartilage defects. Arthritis Rheum. 58, 1067, 2008.

18. Diaz-Romero, J., Nesic, D., Grogan, S.P., Heini, P., and Mainil-Varlet, P. Immunophenotypic changes of human articular chondrocytes during monolayer culture reflect bona fide dedifferentiation rather than amplification of progenitor cells. J. Cell Physiol. 214, 75, 2008.

19. Diaz-Romero, J., Gaillard, J.P., Grogan, S.P., Nesic, D., Trub, T., and Mainil-Varlet, P. Immunophenotypic analysis of human articular chondrocytes: changes in surface markers associated with cell expansion in monolayer culture. J. Cell Physiol. 202, 731, 2005.

20. Kino-Oka, M., Yashiki, S., Ota, Y., Mushiaki, Y., Sugawara, K., Yamamoto, T., Takezawa, T., and Taya, M. Subculture of chondrocytes on a collagen type I-coated 
substrate with suppressed cellular dedifferentiation. Tissue Eng. 11, 597, 2005.

21. Bocelli-Tyndall, C., Barbero, A., Candrian, C., Ceredig, R., Tyndall, A., and Martin, I. Human articular chondrocytes suppress in vitro proliferation of anti-CD3 activated peripheral blood mononuclear cells. J. Cell Physiol. 209, 732, 2006.

22. Pittenger, M.F., Mackay, A.M., Beck, S.C., Jaiswal, R.K., Douglas, R., Mosca, J.D., Moorman, M.A., Simonetti, D.W., Craig, S., and Marshak, D.R. Multilineage potential of adult human mesenchymal stem cells. Science 284, 143, 1999.

23. Grogan, S.P., Barbero, A., Diaz-Romero, J., Cleton-Jansen, A.M., Soeder, S., Whiteside, R., Hogendoom, P.C., Farhadi, J., Aigner, T., Martin, I., and Mainil-Varlet, P. Identification of markers to characterize and sort human articular chondrocytes with enhanced in vitro chondrogenic capacity. Arthritis Rheum. 56, 586, 2007.

24. Nagase, T., Muneta, T., Ju, Y.J., Hara, K., Morito, T., Koga, H., Nimura, A., Mochizuki, T., and Sekiya, I. Analysis of the chondrogenic potential of human synovial stem cells according to harvest site and culture parameters in knees with medial compartment osteoarthritis. Arthritis Rheum. 58, 1389, 2008.

25. Barry, F.P., Boynton, R.E., Haynesworth, S., Murphy, J.M., and Zaia, J. The monoclonal antibody SH-2, raised against human mesenchymal stem cells, recognizes an epitope on endoglin (CD105). Biochem. Biophys. Res. Commun. 265, 134, 1999.

26. Parker, W.L., Goldring, M.B., and Philip, A. Endoglin is expressed on human chondrocytes and forms a heteromeric complex with betaglycan in a ligand and type II TGFbeta receptor independent manner. J. Bone Miner. Res. 18, 289, 2003.

27. Letamendía, A., Lastres, P., Botella, L.M., Raab, U., Langa, C., Velasco, B., Attisano, L., and Bernabeu, C. Role of endoglin in cellular responses to transforming growth factor-beta. A comparative study with betaglycan. J. Biol. Chem. 273, 33011, 1998.

28. Morales, T.I., and Roberts, A.B. Transforming growth factor beta regulates the metabolism of proteoglycans in bovine cartilage organ cultures. J. Biol. Chem. 263, 12828, 1988.

29. Barbero, A., Ploegert, S., Heberer, M., and Martin, I. Plasticity of clonal populations of dedifferentiated adult human articular chondrocytes. Arthritis Rheum. 48, 1315, 2003. 
30. Stokes, D.G., Liu, G., Coimbra, I.B., Piera-Velazquez, S., Crowl, R.M., and Jiménez, S.A. Assessment of the gene expression profile of differentiated and dedifferentiated human fetal chondrocytes by microarray analysis. Arthritis Rheum. 46, 404, 2002.

31. Takahashi, T., Ogasawara, T., Asawa, Y., Mori, Y., Uchinuma, E., Takato, T., and Hoshi, K. Three-dimensional microenvironments retain chondrocyte phenotypes during proliferation culture. Tissue Eng. 13, 1583, 2007.

32. Cukierman, E., Pankov, R., Stevens, D.R., and Yamada, K.M. Taking cell-matrix adhesions to the third dimension. Science 294, 1708, 2001.

33. Yamada, K.M., Pankov, R., and Cukierman, E. Dimensions and dynamics in integrin function. Braz. J. Med. Biol. Res. 36, 959, 2003.

34. Damianova, R., Stefanova, N., Cukierman, E., Momchilova, A., and Pankov, R. Three-dimensional matrix induces sustained activation of ERK1/2 via Src/Ras/Raf signaling pathway. Cell Biol. Int. 32, 229, 2008.

35. Li, T.F., O'Keefe, R.J., and Chen, D. TGF-beta signaling in chondrocytes. Front Biosci 10, 681, 2005.

36. Watanabe, H., de Caestecker, M.P., and Yamada, Y. Transcriptional cross-talk between Smad, ERK1/2, and p38 mitogen-activated protein kinase pathways regulates transforming growth factor-beta-induced aggrecan gene expression in chondrogenic ATDC5 cells. J. Biol. Chem. 276, 14466, 2001. 


\section{FIGURE LEGENDS}

Figure 4.1. In vitro expansion of articular chondrocytes on Plastic, ECM, PtoE or EtoP from P0 to P6. (A) Representative cell morphology from expanded chondrocytes at P0, P1, P3 and P6. (B) Dil-stained chondrocytes (red) with DAPI as a nuclear counterstain (blue) were expanded on either ECM [immunostained with $\left(B_{i}\right)$ or without $\left(B_{i i}\right)$ collagen I antibody (green)] or Plastic ( $B_{\text {iii) }}$. (C) Fold-change of expanded cell number was compared for plating on either ECM or Plastic. (D) Fold-change of collagen II (Col II) mRNA was compared in cartilage tissue and chondrocytes expanded on ECM or Plastic. (E) Fold-change of collagen I (Col I) mRNA was compared in cartilage tissue and chondrocytes expanded on ECM or Plastic. Data are shown as average \pm SD for $n=4$.

Figure 4.2. Immunophenotype changes of chondrocytes expanded on ECM, Plastic, PtoE, or EtoP from P0 to P6. Flow cytometry was used to evaluate the percentage ( $\mathrm{A}-\mathrm{C}$ ) and mean fluorescence intensity (MFI, a - c) of CD14+, CD90+, and CD105+ chondrocytes during cell expansion. Dual positive chondrocytes of CD90+ and CD105+ were represented at P0, P2 and P6 (D). Data are shown as average \pm SD for $n=3$.

Figure 4.3. Redifferentiation capacity of expanded chondrocytes was assessed at the protein level using chondrocyte-pellets after incubation in a TGF- $\beta 1$-containing medium for 14 days. (A) Alcian blue staining was for sulfated GAGs with fast red as a counterstain, and immunohistochemistry (IHC) was for collagens I and II (DAB substrate had a brown color suggestive of positive staining) with hematoxylin as a counterstain. The scale bar was 800 $\mu \mathrm{m}$. (B) Western blot was used to detect collagen II in extracellular matrix (mature) and cytoplasm (immature) with $\beta$-actin as an internal control. (C) Biochemical analysis was used to quantitatively measure DNA and GAG amounts in the pellets. DNA data were represented by DNA ratio adjusted by DNA amount at day 0 . Ratio of GAG to DNA was used as a chondrogenic index. Data are shown as average \pm SD for $n=5$. Groups not connected by the same letter are significantly different $(p<0.05)$. 
Figure 4.4. Redifferentiation capacity of expanded chondrocytes was assessed at the mRNA level using chondrocyte-pellets after incubation in a TGF- $\beta 1$-containing medium for 14 days. TaqMan ${ }^{\circ}$ RT-PCR was used to quantitatively measure chondrogenic marker genes (A - collagen II, aggrecan, and collagen I) and hypertrophic marker genes (B - collagen X, ALP, and MMP13) in the pellets of expanded chondrocytes at days 0 and 14. Data are shown as average $\pm S D$ for $n=5$. Groups not connected by the same letter are significantly different $(p<0.05)$.

Figure 4.5. Redifferentiation capacity of expanded chondrocytes was assessed at the protein level using chondrocyte-pellets after incubation in a serum-containing medium for 14 days. (A) Alcian blue staining was used for sulfated GAGs with fast red as a counterstain, and immunohistochemistry (IHC) was for collagens I and II (DAB substrate had a brown color suggestive of positive staining) with hematoxylin as a counterstain. The scale bar was 800 $\mu \mathrm{m}$. (B) Western blot was used to detect collagen II in extracellular matrix (mature) and cytoplasm (immature) of pellets with $\beta$-actin as an internal control at days 7 and 14. (C) Biochemical analysis was used to quantitatively measure DNA and GAG amounts in the 7and 14-day pellets. DNA data were represented by DNA ratio adjusted by DNA amount at day 0 . Ratio of GAG to DNA was used as a chondrogenic index. Data are shown as average \pm SD for $n=5$. Groups not connected by the same letter are significantly different $(p<0.05)$.

Figure 4.6. Redifferentiation capacity of expanded chondrocytes was assessed at the mRNA level using chondrocyte-pellets after incubation in a serum-containing medium for 14 days. TaqMan® RT-PCR was used to quantitatively measure chondrogenic marker genes (collagen II, aggrecan, and collagen I) in the pellets of expanded chondrocytes at days 7 and 14. Data are shown as average \pm SD for $n=5$. Groups not connected by the same letter are significantly different $(p<0.05)$.

Figure 4.S1. Redifferentiation capacity of expanded chondrocytes was assessed at the protein and mRNA level using chondrocyte-pellets after incubation in a TGF- $\beta 1$ - or serum-containing medium at Day 7. 
Figure 4.S2. Redifferentiation capacity of expanded chondrocytes was assessed at the protein and mRNA level using chondrocyte-pellets after incubation in a TGF- $\beta 1$-containing medium at Day 7. (A) Western blot was used to detect collagen II in extracellular matrix (mature) and cytoplasm (immature) of pellets with $\beta$-actin as an internal control at Day 7 in the group of TGF- $\beta 1$ treatment. (B) Biochemical analysis was used to quantitatively measure DNA and GAG amounts in the 7-day pellets treated by TGF- $\beta 1$. (C) mRNA level using chondrocyte-pellets after incubation in a TGF- $\beta 1$-containing medium at Day 7. 
Figure 4.1

A

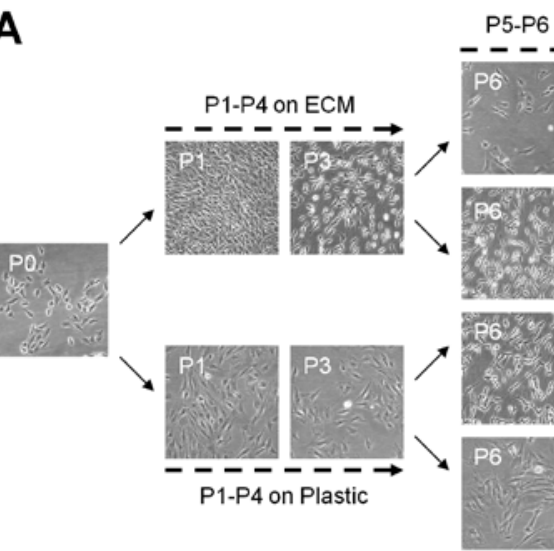

B

EtoP

ECM

Plastic

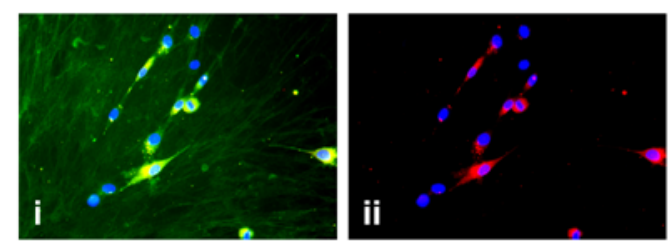

C

D
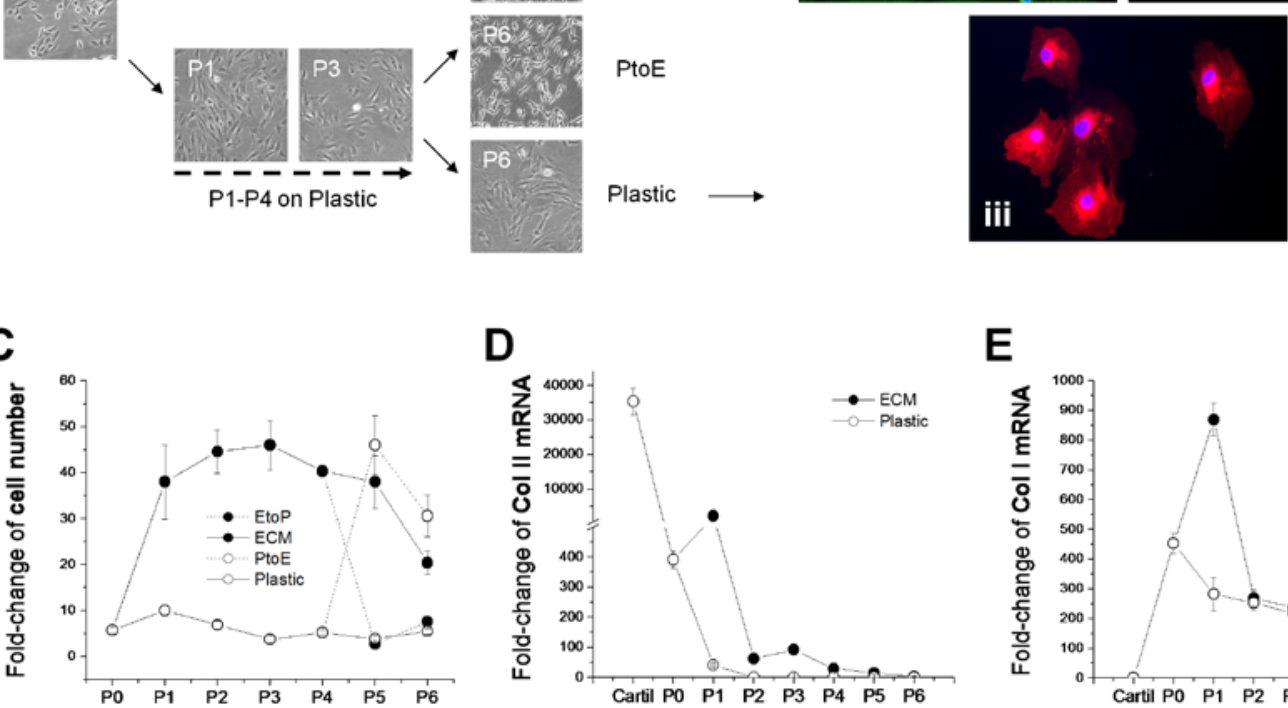

E

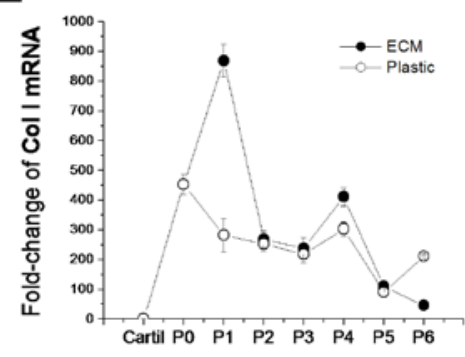


Figure 4.2

A
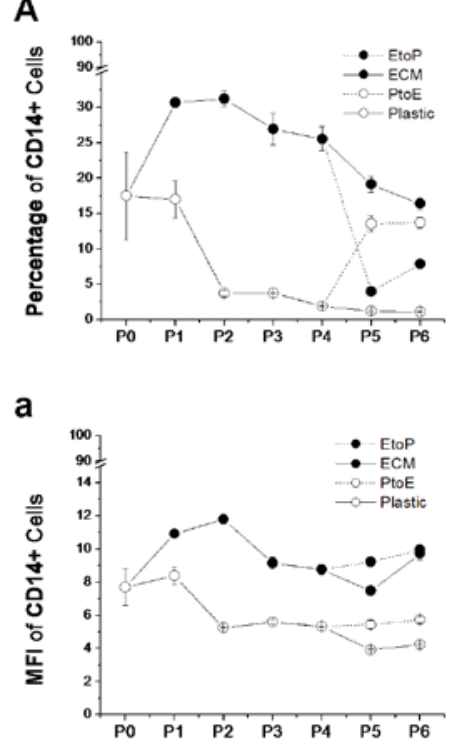

D

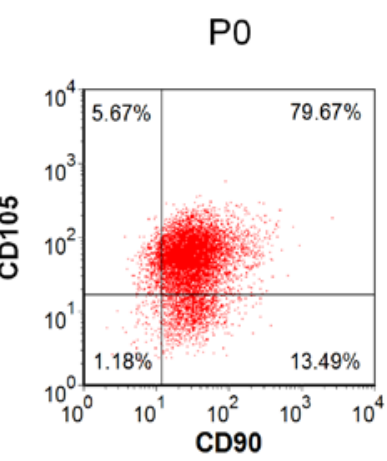

B

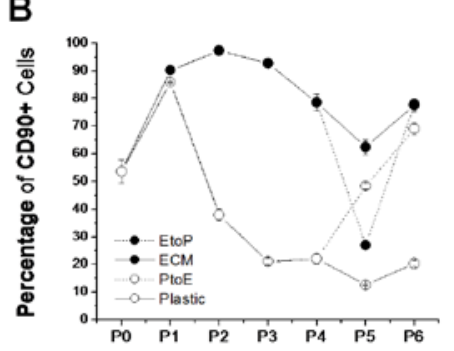

b

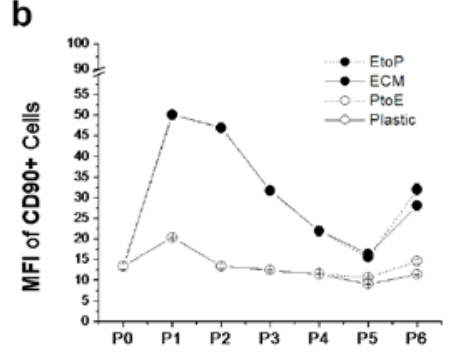

P2
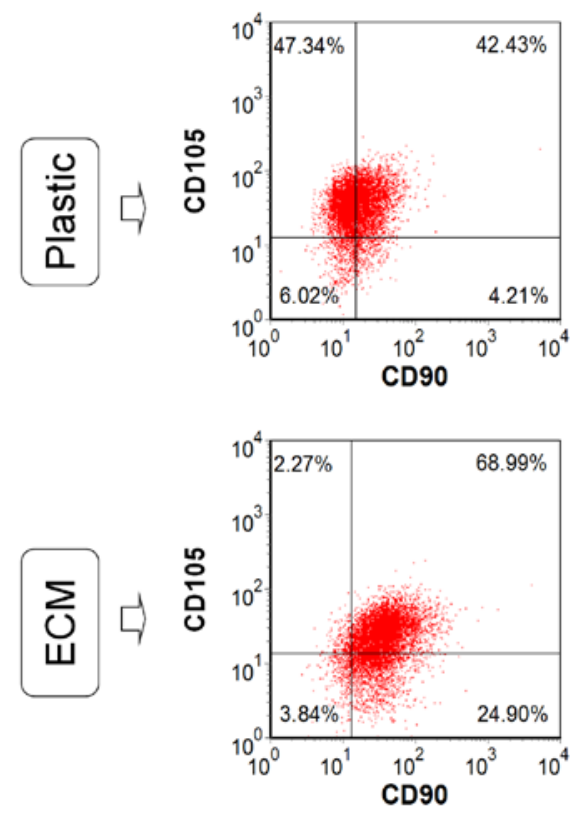
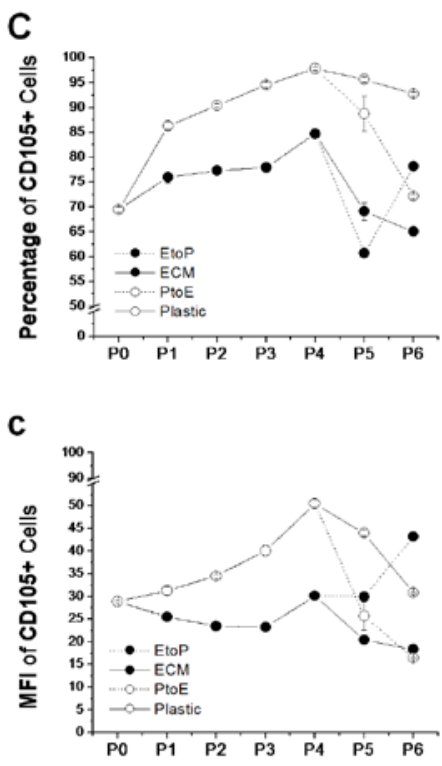

P6
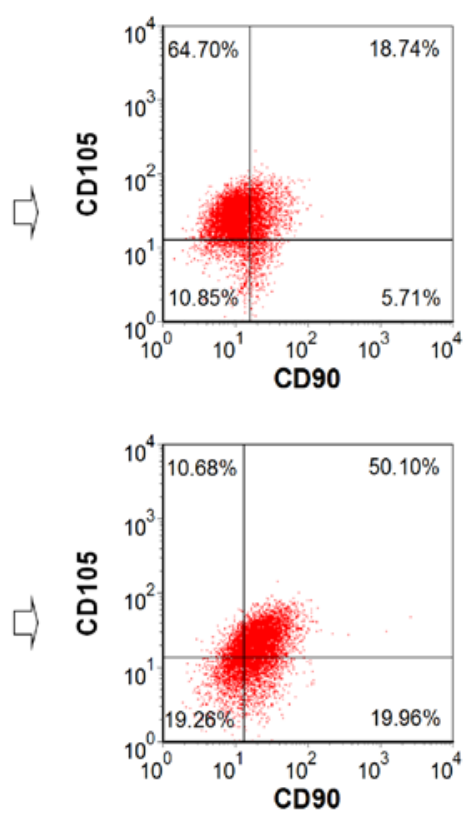
Figure 4.3

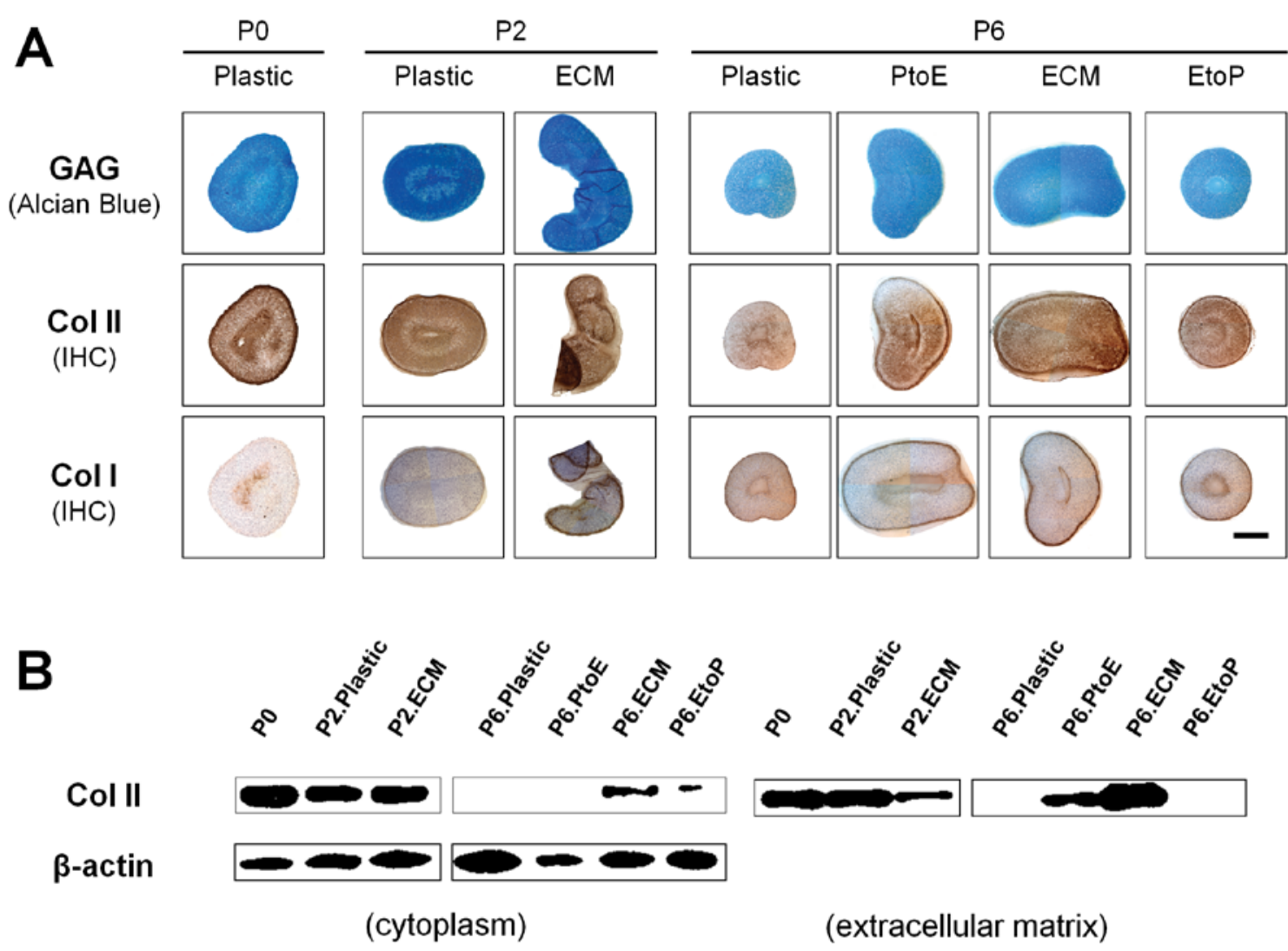

C
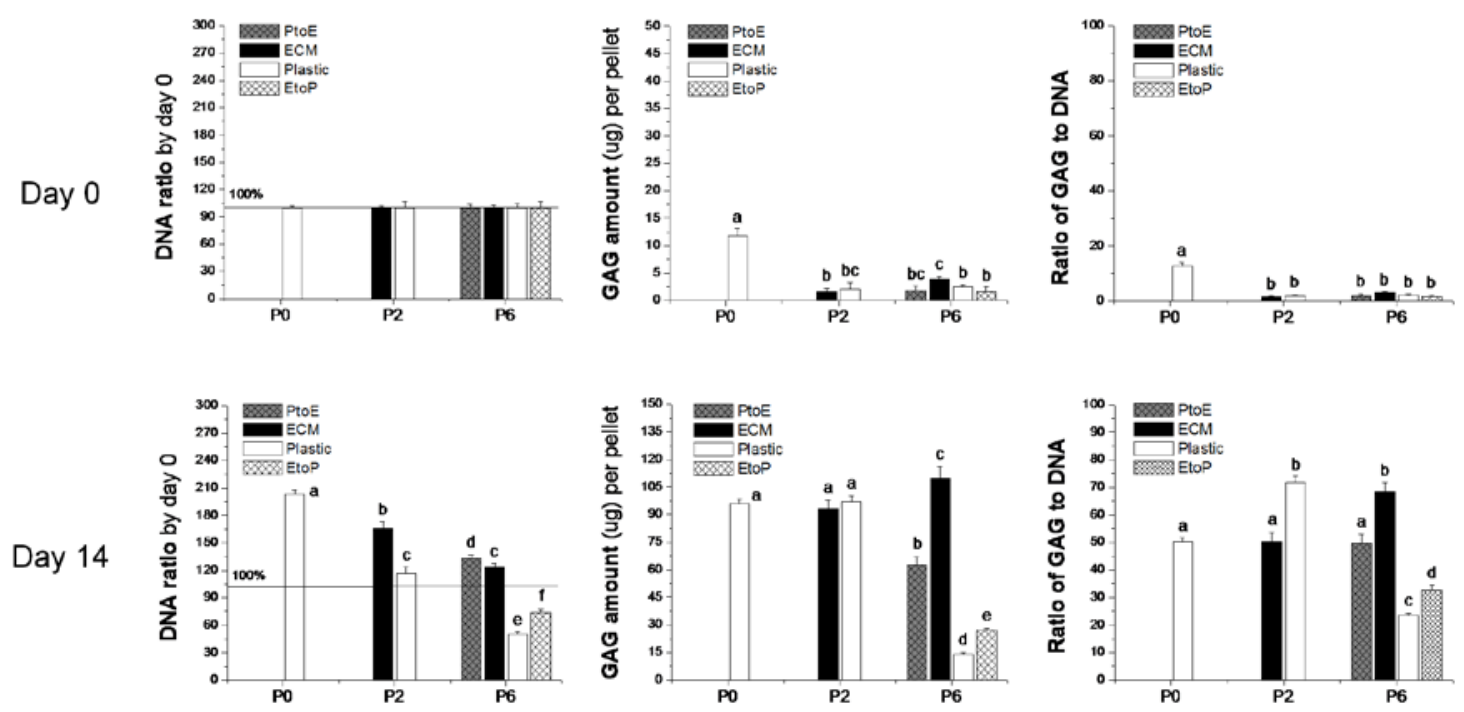
Figure 4.4

A

Collagen II

Aggrecan

Collagen I
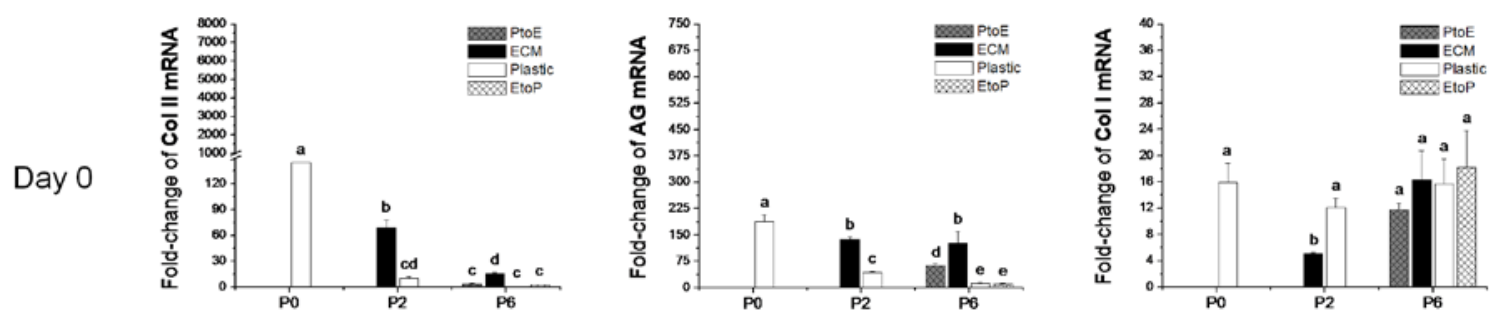

Day 14
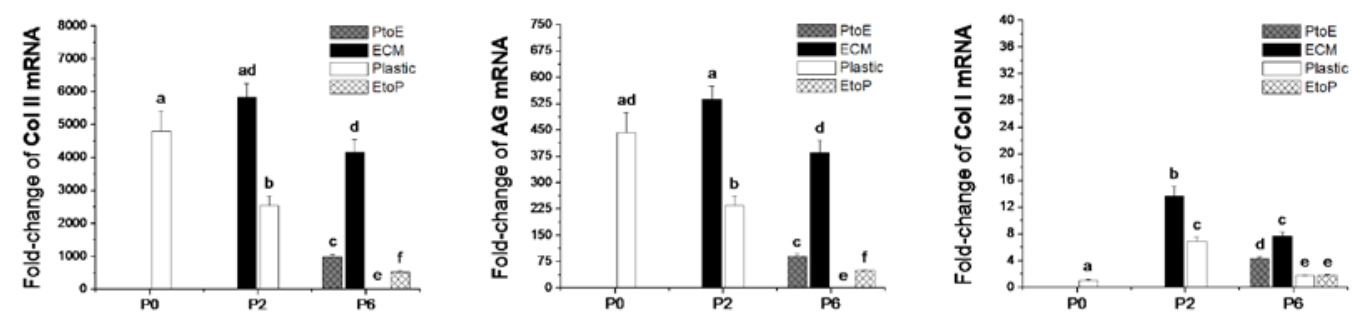

B

Collagen $X$

ALP

MMP13
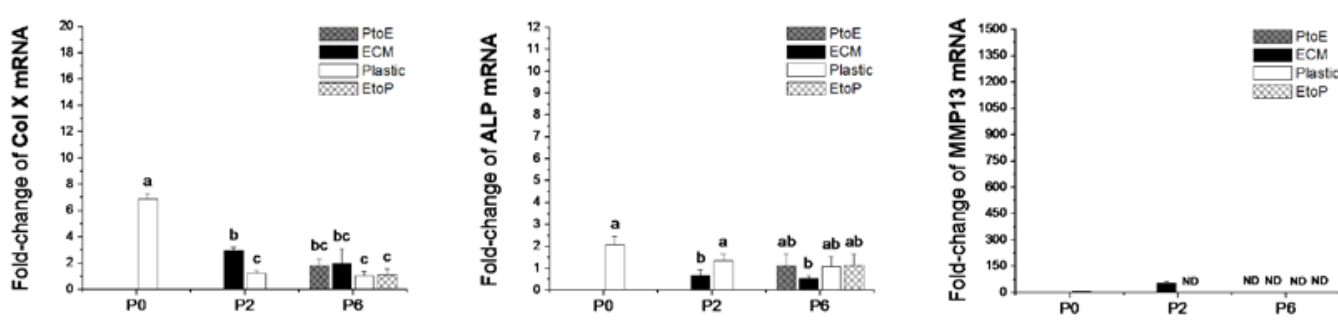

Day 14
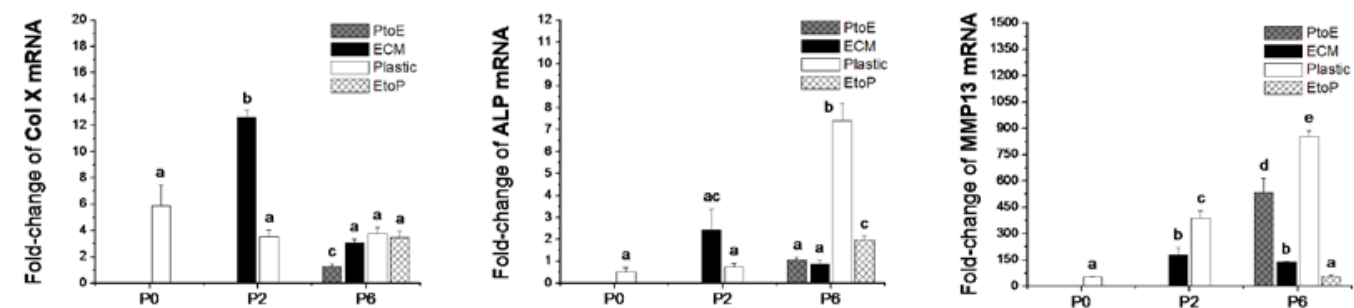
Figure 4.5

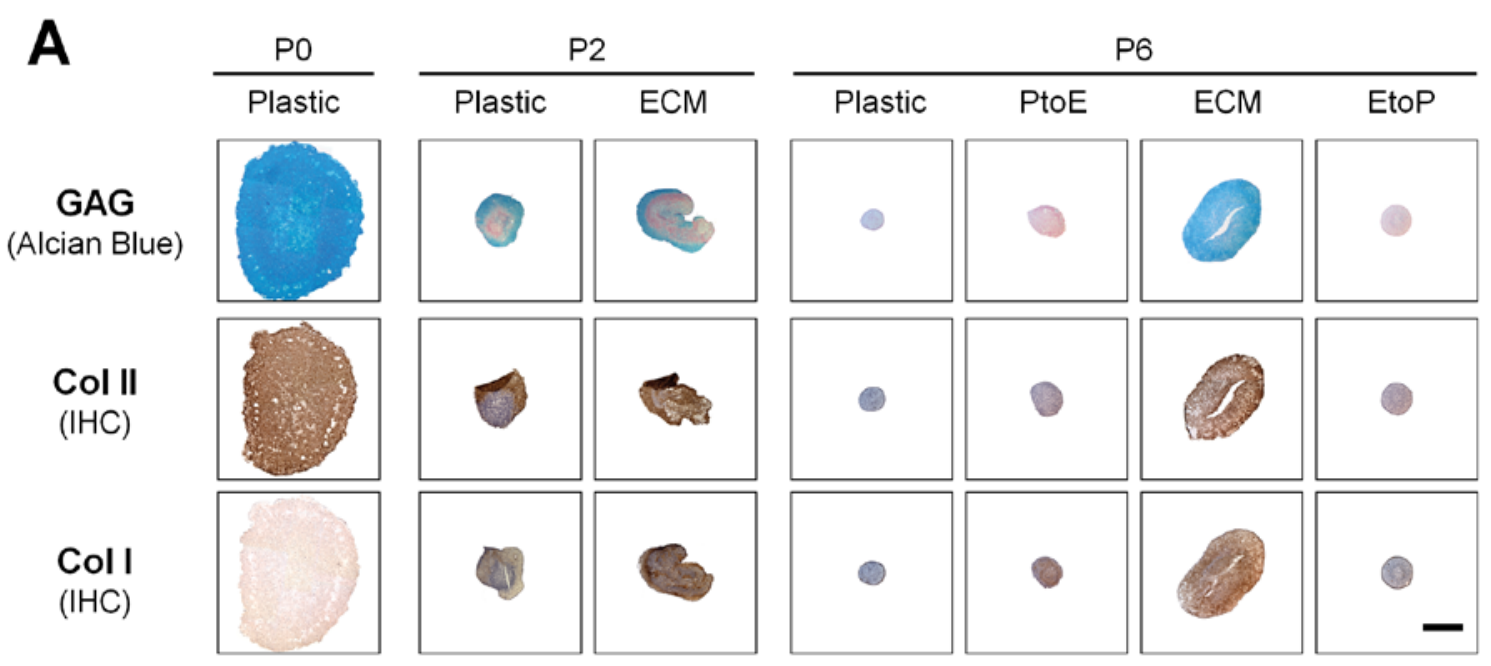

B Day 7

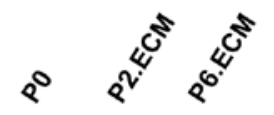

$e^{\circ}$

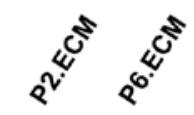

Col II

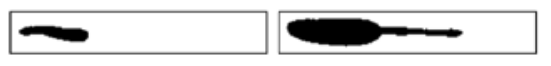

$\beta$-actin

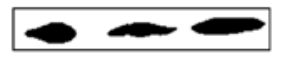

(cytoplasm) (extracellular matrix)

Day 14
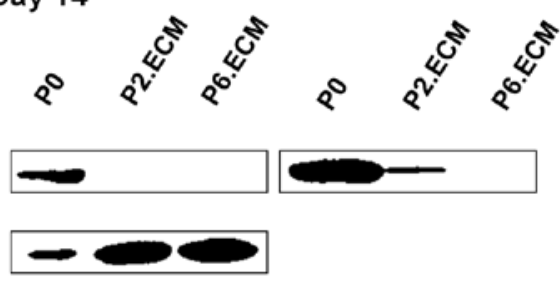

(cytoplasm) (extracellular matrix)

C

Day 7
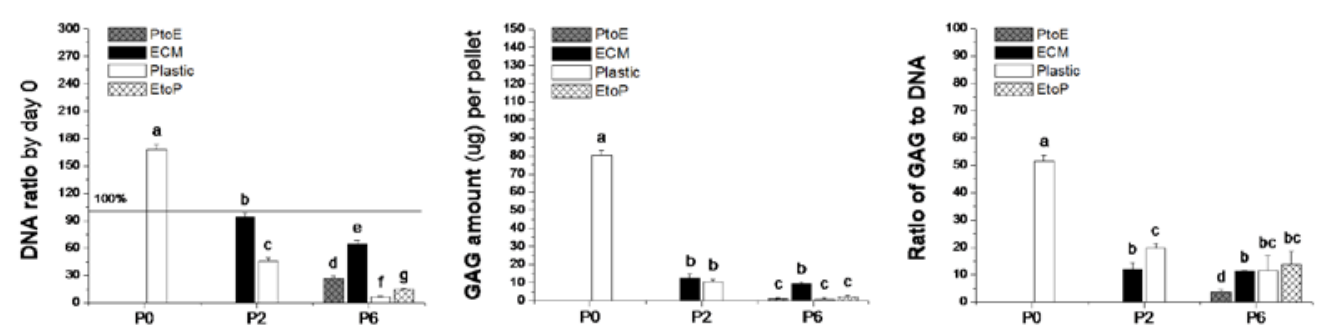

Day 14
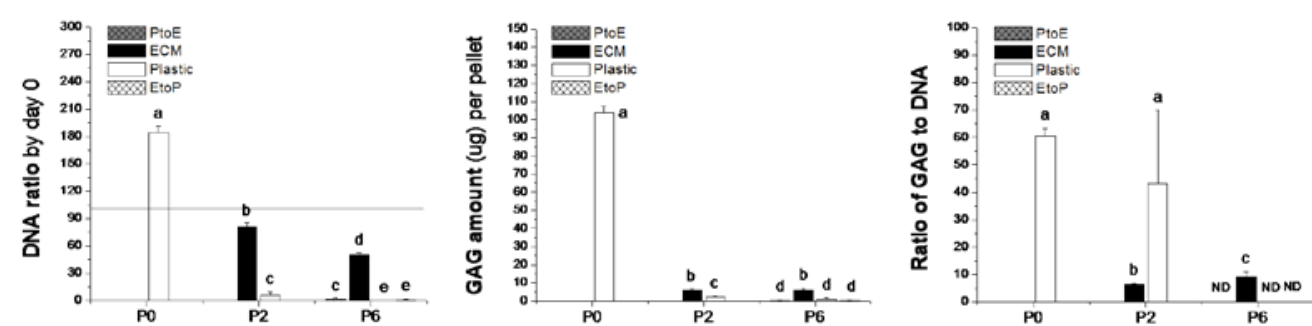
Figure 4.6

Collagen II
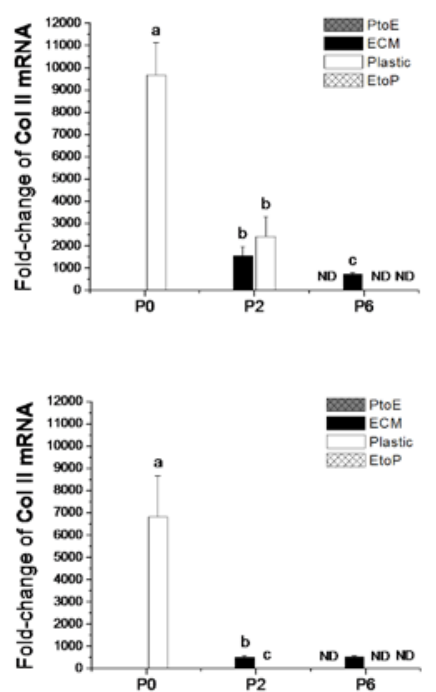

Aggrecan
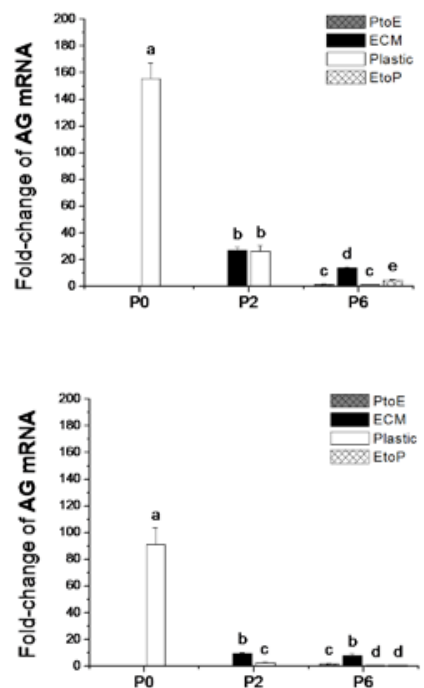

Collagen I
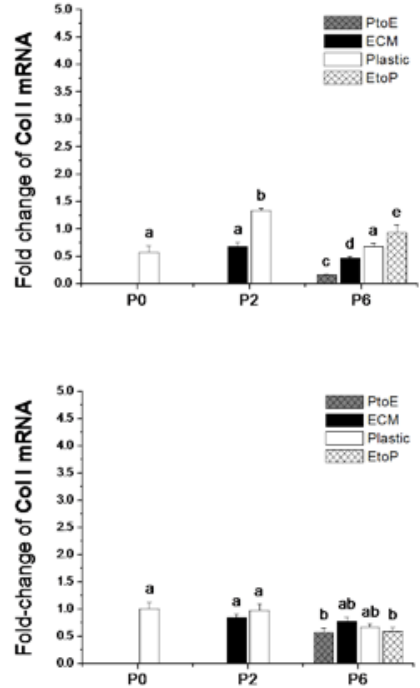
Figure 4.S1

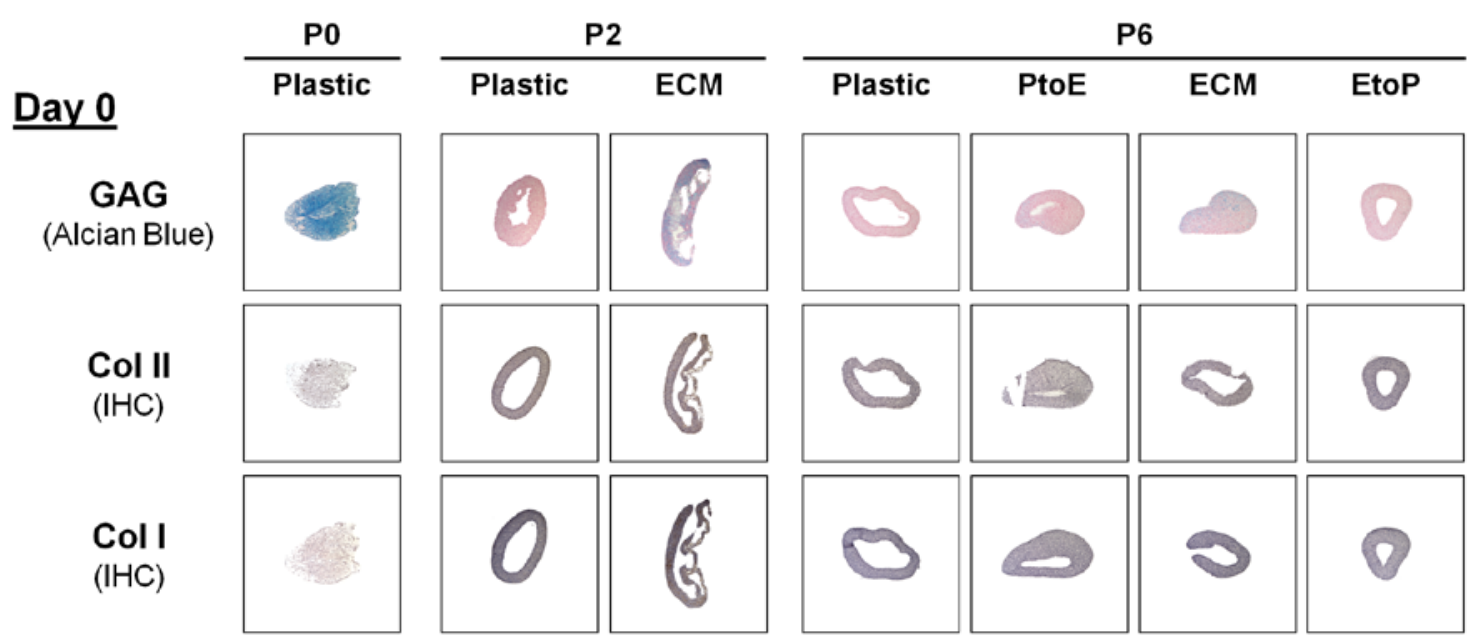

\section{Day 7}

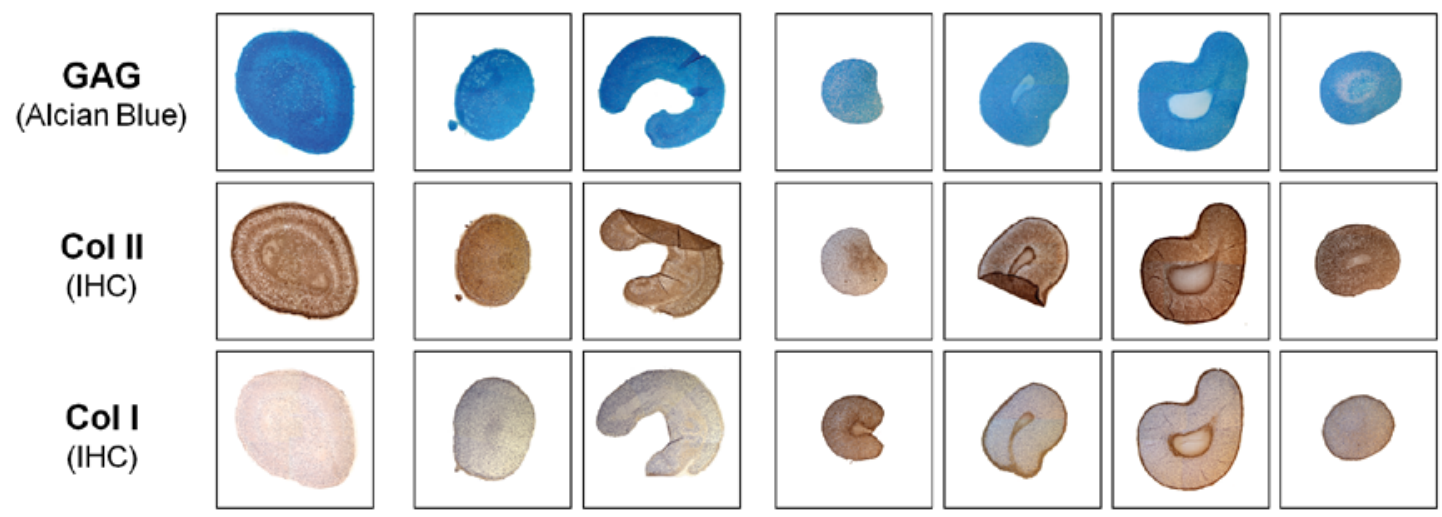

Serum-containing medium

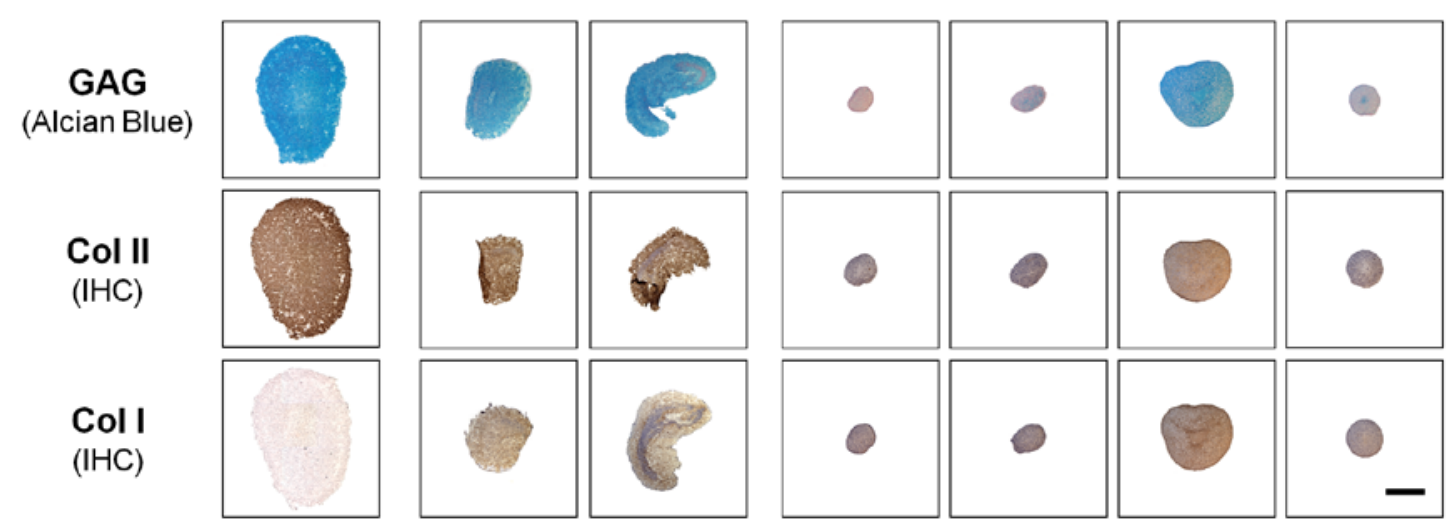


Figure 4.S2

A

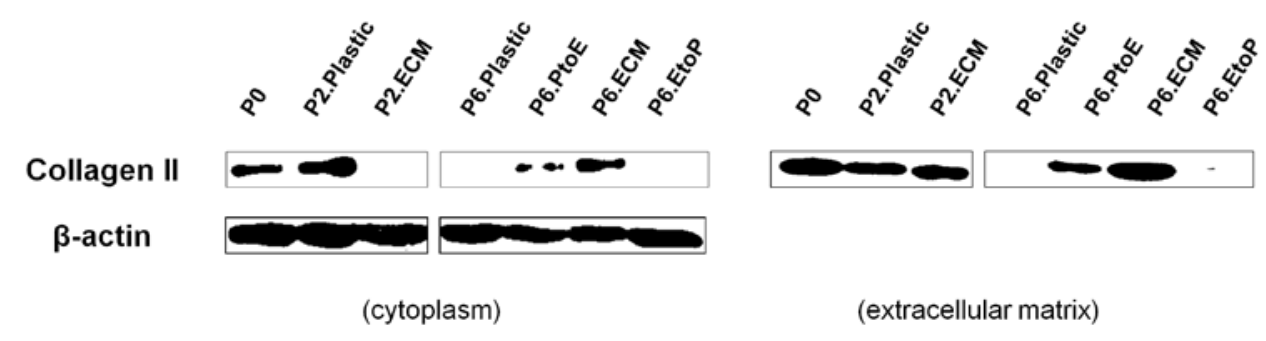

B
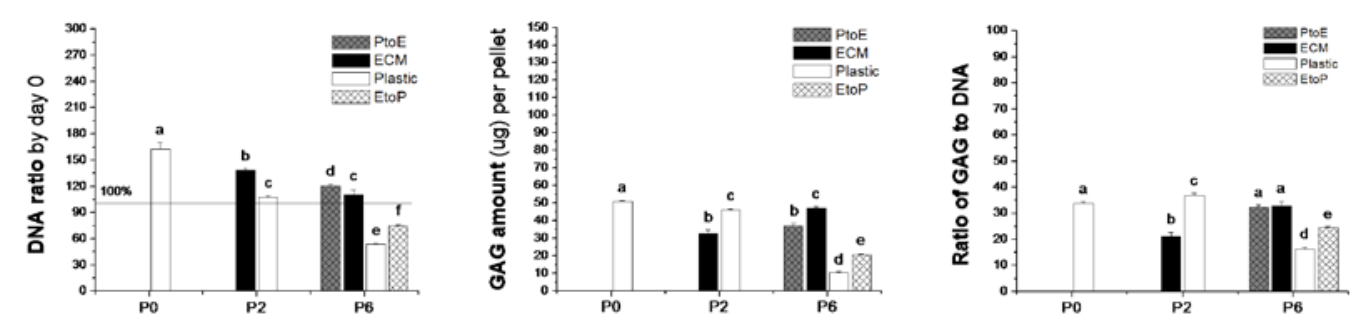

C
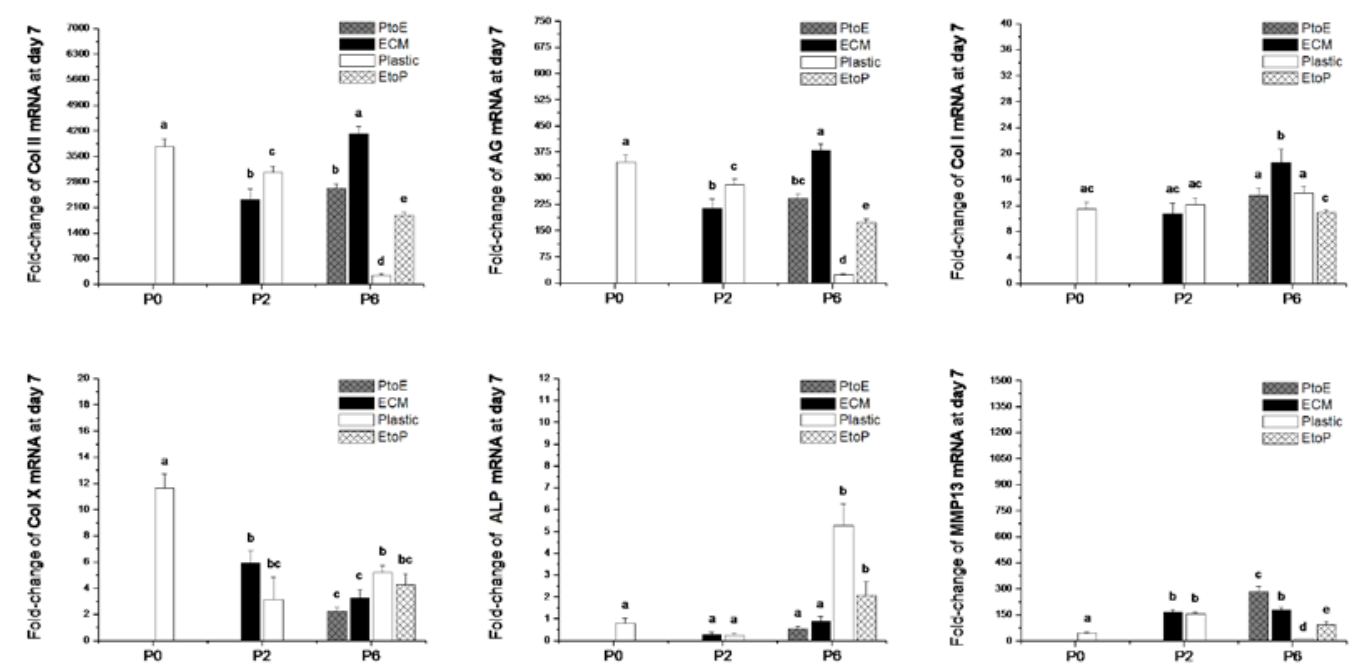


\title{
Chapter 5
}

\section{Rejuvenation of Nucleus Pulposus Cells Using Extracellular Matrix Deposited by Synovium-Derived Stem Cells}

\author{
Fan He, BS, and Ming Pei, MD, PhD
}

Stem Cell and Tissue Engineering Laboratory, Department of Orthopaedics and Division of Exercise Physiology, West Virginia University, Morgantown, West Virginia 26506 


\begin{abstract}
Study Design. After plating for six passages on either plastic flasks or extracellular matrix (ECM) deposited by synovium-derived stem cells (SDSCs), expanded nucleus pulposus (NP) cells were evaluated for redifferentiation capacity.

Objective. The aim was to assess the feasibility of using ECM deposited by a tissue-specific stem cell to provide a 3D microenvironment for NP cell rejuvenation.

Summary of Background Data. Autologous disc cell-based therapy is a promising approach for intervertebral disc (IVD) regeneration. Unfortunately, the current in vitro expansion of NP cells in monolayer results in dedifferentiation of these cells.

Methods. Primary NP cells were plated on either plastic flasks or ECM for six consecutive passages. At each passage, cell numbers were counted for proliferation rate, cell phenotype was evaluated using flow cytometry and cell differentiation status was assessed using real-time PCR. The pellets from expanded NP cells at passages 1, 4 and 6 were incubated in a serum-free defined medium for 14 days. Redifferentiation capacity of the expanded NP cells was evaluated using histology, biochemistry, and real-time PCR.

Results. NP cells expanded on ECM grew much faster with a smaller size and fibroblast-like shape compared to those on plastic flasks. ECM-treated NP cells acquired an enhanced CD90 expression and higher mRNA levels of types I, II, and X collagen and aggrecan, as well as a robust redifferentiation capacity, evidenced by dramatically increased type II collagen, aggrecan and Sox9 and decreased type I collagen for up to six passages.

Conclusion. SDSC-derived ECM can provide a tissue-specific microenvironment for the rejuvenation of NP cells with a higher proliferation rate and redifferentiation capacity, which may play a role in improving an autologous disc cell-based minimally invasive therapeutic approach toward physiological reconstruction of a biologically functional disc in the clinical setting.
\end{abstract}

Key words: 
Extracellular matrix, stem cell niche, proliferation, dedifferentiation, redifferentiation, synovium, nucleus pulposus cell

Key points:

- Monolayer culture can result in NP cell dedifferentiation, evidenced by losing cell proliferation and gene expression profiles as well as redifferentiation capacity

- ECM deposited by SDSCs provides a tissue-specific 3D microenvironment for NP cell large-scale expansion

- NP cells expanded on ECM acquire a higher expression of CD90 and differentiated gene profiles

- NP cells expanded on ECM also acquire a robust redifferentiation capacity

- The expansion microenvironment switched from plastic flasks to ECM can improve NP cell rejuvenation 


\section{INTRODUCTION}

Approximately $80 \%$ of the adult population is affected by low back pain (LBP); this has a massive economic impact due to both health care costs and loss of productivity. ${ }^{1}$ Although the causes of LBP are complicated and not fully understood, degeneration of the intervertebral disc (IVD) is an important underlying cause. ${ }^{2 ; 3}$ IVD degeneration begins in the nucleus pulposus (NP) with a progressive decrease in proteoglycan content, leading to a loss of hydrogel properties of the NP. ${ }^{4}$ Clinical treatments of IVD disease include various noninvasive and invasive therapies, but are limited to symptomatic relief. Much of the recent research in this area has focused on biological restoration of the IVD. ${ }^{5 ; 6}$ Although human IVD cells share matrix-producing properties with chondrocytes, studies have shown that there are indeed phenotypic differences between NP cells and articular chondrocytes. ${ }^{7}$ Stem cells seem to be another candidate for cell transplantation; however, the lack of a definitive cell marker to distinguish NP cells from other cells has made clinical application more difficult and less realistic.

Animal studies ${ }^{8-10}$ and an early clinical trial (Euro Disc Randomized Trial) ${ }^{11}$ have indicated that autologous disc cell transplantation (ADCT) is technically feasible and biologically relevant to repairing disc damage and retarding disc degeneration. However, one concern of critics of ADCT is that cells isolated from degenerative discs may lack the potential of healthy counterparts, making them poorly suited for repair in an inhospitable degenerated disc environment. ${ }^{12}$ In addition, the preparation of NP cells for reimplantation is problematic because autologous transplantation requires more cells than can be harvested from a small biopsy. With low cellular yields and low proliferative activity of NP cells in monolayer culture, further enhancement of the biological and metabolic viability of NP cells is desirable.

Our recent study has shown that synovium-derived stem cells (SDSCs) can be used to prepare a 3D microenvironment for SDSC proliferation while maintaining and enhancing their chondrogenic differentiation capacity. ${ }^{13}$ Since NP cells are chondrocyte-like cells, in this study, we hypothesize that SDSC-derived ECM can serve as a tissue-specific microenvironment for 
ex vivo expansion of NP cells while maintaining NP cell differentiated phenotype and redifferentiation capacity. Our long-term goal is to develop an autologous disc cell-based minimally invasive therapeutic approach toward physiological reconstruction of a biologically functional disc in the clinical setting. 


\section{METHODS}

Isolation and culture of SDSCS and NP cells

Two 3-month-old pigs were collected from a local slaughterhouse and harvested to provide synovial tissue from both knees and NP tissue from the lumbar spine $\left(L_{1}-L_{5}\right)$. The synovial tissue was finely minced and digested at $37^{\circ} \mathrm{C}$ for $30 \mathrm{~min}$ in $0.1 \%$ trypsin (Roche, Indianapolis, $\mathrm{IN}$ ) and then for $2 \mathrm{~h}$ in $0.1 \%$ collagenase $\mathrm{P}$ (Roche) to release synovial cells. The NP tissue was digested with $0.1 \%$ collagenase A (Sigma, St. Louis, MO) and $10 \mathrm{U} / \mathrm{mL}$ hyaluronidase (Sigma) at $37^{\circ} \mathrm{C}$ for $4 \mathrm{~h}$. Synovial cells and NP cells were collected from the filtrate by centrifugation and plated in complete medium [ $\alpha$ MEM containing $10 \%$ fetal bovine serum (FBS), $100 \mathrm{U} / \mathrm{mL}$ penicillin, $100 \mu \mathrm{g} / \mathrm{mL}$ streptomycin and $0.25 \mu \mathrm{g} / \mathrm{ml}$ fungizone]. Synovial fibroblasts were negatively isolated from primary culture and characterized as SDSCS according to our previous study ${ }^{14}$.

\section{Preparation of SDSC-derived ECM}

The preparation of SDSC-derived ECM was described in our previous study. ${ }^{13}$ Briefly, fibronectin was pre-coated on plastic flasks at $37^{\circ} \mathrm{C}$ for $1 \mathrm{~h}$. When the plated SDSCs reached 90\% confluence, $50 \mu \mathrm{M}$ L-ascorbic acid phosphate (Wako, Richmond, VA) was added for eight days. ECM was washed with phosphate buffered saline (PBS), and incubated with 0.5\% Triton X-100 containing $20 \mathrm{mM}$ ammonium hydroxide in PBS at $37^{\circ} \mathrm{C}$ for $5 \mathrm{~min}$ and stored in PBS.

NP cells grown on SDSC-derived ECM

Passage one (P1) NP cells were plated at 3,000 cells $/ \mathrm{cm}^{2}$ in plastic flasks with ("ECM") or without ECM ("Plastic") -coating for six consecutive passages. The third group was grown on plastic flasks for five passages and then switched on ECM for one passage ("PtoE"). Complete medium was replaced every three days. Cell number was quantified using a counting hemocytometer. 


\section{FACS analysis}

Triple individual samples of $0.3 \times 10^{6}$ cells each were incubated on ice in PBS containing 0.1\% ChromPure Swine IgG (Jackson Immuno Research, West Grove, PA) and $1 \% \mathrm{NaN}_{3}$ for 30 min. Primary monoclonal antibodies were applied for 30 min, including CD29 (Abcam, Cambridge, MA), CD44 (Abcam), CD90 (BD Pharmingen, San Jose, CA), CD105 (GeneTex Inc., San Antonio, TX) and isotype-matched IgGs (Beckman Coulter, Fullerton, CA). After washing with cold PBS, NP cells were incubated with the secondary antibody [FITC-conjugated goat anti-mouse Ig (Abcam)] for $30 \mathrm{~min}$ in the dark followed by fixation in $400 \mu \mathrm{L}$ of $0.4 \%$ paraformaldehyde. NP cells were analyzed on a BD dual laser FACS Calibur (BD Biosciences) using the FCS Express Version 3 (De Novo Software, Los Angeles, CA) software package.

\section{Redifferentiation of expanded NP cells}

$0.3 \times 10^{6}$ of NP cells from P1, P4, and P6 were centrifuged at $500 \mathrm{~g}$ for $5 \mathrm{~min}$ to form a pellet. After $24 \mathrm{~h}$ incubation (day 0 ), the pellets were cultured in a defined medium consisting of high-glucose DMEM, $40 \mu \mathrm{g} / \mathrm{mL}$ proline, $100 \mathrm{nM}$ dexamethasone, $100 \mathrm{U} / \mathrm{mL}$ penicillin, 100 $\mu \mathrm{g} / \mathrm{mL}$ streptomycin, $0.1 \mathrm{mM}$ ascorbic acid-2-phosphate and $1 \times$ ITS $^{\text {TM }}$ Premix (BD Biosciences) with $10 \mathrm{ng} / \mathrm{mL}$ transforming growth factor $\beta 1$ (TGF- $\beta 1$ ) (R\&D Systems, Minneapolis, MN) for 14 days.

\section{Histochemistry and immunohistochemistry}

The pellets $(n=3)$ were fixed in $4 \%$ paraformaldehyde, dehydrated in a gradient ethanol series, cleared with xylene, and embedded in paraffin blocks. $5-\mu \mathrm{m}$ sections were histochemically stained with Safranin-O (Sigma). For immunohistochemical analysis, the sections were immunolabeled with primary antibodies against collagen I (Sigma) and collagen II (II-II6B3, DSHB, lowa City, IA), respectively. A secondary antibody of biotinylated horse anti-mouse IgG (Vector, Burlingame, CA) was incubated on the sections for 30 min. Immunoactivity was detected using Vectastain $A B C$ reagent (Vector) with 3,3'-diaminobenzidine (DAB) as a substrate. Hematoxylin (Vector) served as a counterstain. 
Biochemical analysis for DNA and GAG content

The pellets $(n=6)$ were digested for $4 \mathrm{~h}$ at $60^{\circ} \mathrm{C}$ with $125 \mu \mathrm{g} / \mathrm{mL}$ papain in PBE buffer (100 $\mathrm{mmol} / \mathrm{L}$ phosphate, $10 \mathrm{mmol} / \mathrm{L}$ EDTA, $\mathrm{pH}$ 6.5) containing $10 \mathrm{mmol} / \mathrm{L}$ cysteine using $100 \mu \mathrm{L}$ enzyme per sample. To quantify cell density, the amount of DNA in the papain digestion was measured using the QuantiT ${ }^{\mathrm{TM}}$ PicoGreen $^{\circledR}$ dsDNA assay kit (Invitrogen) with a CytoFluor ${ }^{\circledR}$ Series 4000 (Applied Biosystems, Foster City, CA). GAG was measured using dimethylmethylene blue dye and a Spectronic ${ }^{\mathrm{TM}}$ BioMate $^{\mathrm{TM}} 3$ Spectrophotometer with bovine chondroitin sulfate as a standard.

\section{TaqMan ${ }^{\circledR}$ Quantitative PCR}

The total RNA was extracted from samples $(n=5)$ using an RNase-free pestle in TRIzol ${ }^{\circledR}$ (Invitrogen). $1 \mu \mathrm{g}$ of mRNA was used for reverse transcriptase (RT) with a High-Capacity cDNA Archive Kit at $37^{\circ} \mathrm{C}$ for $120 \mathrm{~min}$ as recommended by the manufacturer (Applied Biosystems). Chondrogenic marker genes (types I, II, and X collagen, Sox9, and aggrecan) were customized by Applied Biosystems as part of the Custom Taqman ${ }^{\circledR}$ Gene Expression Assays. ${ }^{13}$ Eukaryotic 18S RNA (Assay ID: HS99999901_s1 ABI) was carried out as the endogenous control gene. Real-time PCR was performed with the iCycler iQ ${ }^{\mathrm{TM}}$ Multi Color RT-PCR Detection and calculated by computer software (Perkin-Elmer, Waltham, MA). Relative transcript levels were calculated as $\mathrm{X}=2^{-\Delta \Delta \mathrm{Ct}}$, in which $\Delta \Delta \mathrm{Ct}=\Delta \mathrm{E}-\Delta \mathrm{C}, \Delta \mathrm{E}=\mathrm{Ct}_{\mathrm{exp}}-\mathrm{Ct}_{18 \mathrm{~s}}$, and $\Delta \mathrm{C}=\mathrm{Ct}_{\mathrm{ct1} 1}-\mathrm{Ct}_{18 \mathrm{~s}}$.

\section{Statistics}

The Kruskal-Wallis test was used to test for significant differences among all groups and the Mann-Whitney $U$ test was used for pairwise comparison in flow cytometry data, biochemistry analysis, and real-time PCR data. All statistical analyses were performed with SPSS 13.0 statistical software (SPSS Inc., Chicago, IL). $P$ values less than 0.05 were considered statistically significant. 


\section{RESULTS}

SDSC-derived ECM enhances NP cell proliferation while retaining their differentiation phenotypes

Primary NP cells were plated in plastic flasks without or with ECM coating for six passages. NP cells plated on plastic flasks were shown to proliferate slowly; in contrast, NP cells grown on ECM had their cell number increased dramatically by overlapping in a 3D format after reaching confluence (Figure 5.1A). Relative to NP cells expanded on plastic flasks, ECM-treated NP cells yielded 7.8-fold greater cell numbers at P1. Despite a decreased proliferation rate with passage in both groups, NP cells expanded on ECM yielded 10.8-fold at P2, 19.1-fold at P3, 13.9-fold at P4, and 15.0-fold at P6 greater cell numbers than those plated on plastic flasks (Figure 5.1B).

To identify if NP cells expanded on ECM acquired mesenchymal stem cell (MSC) phenotypes, CD29, CD44 and CD90 were assessed using flow cytometry (Figure 5.2A). Despite a comparable percentage of CD29+ cells (Figure 5.2B), NP cells expanded on ECM were lower in mean fluorescent intensity (MFI) than those on plastic flasks (Figure 5.2C). Interestingly, NP cells expanded on ECM acquired CD90+ cells greater than those grown on plastic flasks in both percentage (Figure 5.2D) and MFI (Figure 5.2E), indicating that SDSC-derived ECM provides a 3D microenvironment to enhance NP cell "stemness" toward chondrogenic differentiation. ${ }^{15}$ For CD44, ECM-treated NP cells shared a similar expression phenotype as those grown on plastic flasks except slightly higher at P3 and P6 in both percentage (Figure 5.2F) and MFI (Figure 5.2G). Noticeably, NP cells expanded from PtoE acquired immunophenotypes close to those on ECM, particularly in MFI (Figure 5.2C, 5.2E, 5.2G).

To identify differentiation status of expanded NP cells, types I, II, and X collagen and aggrecan were assessed using real-time PCR. Chondrogenic marker genes [collagen II (Figure 5.3A) and aggrecan (Figure 5.3B)] decreased their expression with passage, particularly in the early subculture of NP cells on plastic flasks. In contrast, ECM-treated NP cells showed greater collagen II and aggrecan mRNA in all passages than those seeded on 
plastic flasks. Intriguingly, ECM-treated NP cells also exhibited higher collagen I mRNA (Figure 5.3C) and collagen $X$ mRNA (Figure 5.3D) than those grown on plastic flasks. Collagen I mRNA was elevated in the initial passage followed by a gradual decrease until P6 in both groups.

In vitro tissue-specific microenvironment restores redifferentiation capacity of expanded NP cells

Despite no positive staining at day 0 , the pellets from expanded NP cells on either plastic flasks or ECM at P1 were stained intensively with sulfated GAGs and types I and II collagen at day 7 while type I collagen became weaker with the maturation of pellets. Fourteen-day-pellets from NP cells expanded on ECM were larger in size and more homogeneous in the distribution of sulfated GAGs and type II collagen than those on plastic flasks. More evidently, ECM-treated NP cells at P4 yielded pellets with more sulfated GAGs and types I and II collagen at day 7 than those grown on plastic flasks. ECM-treated NP cells at P6 yielded pellets with more intense staining of sulfated GAGs and collagen II at days 7 and 14 than those from NP cells expanded on plastic flasks. The pellets of NP cells expanded from PtoE exhibited more intense expression of collagen II and sulfated GAGs than those on plastic flasks (Figure 5.4).

To evaluate redifferentiation capacity of expanded NP cells, biochemical analysis was used to quantify GAG amount, DNA amount and ratio of GAG to DNA (chondrogenic index) in pellets. Adjusted by those at day 0 , pellets from P1 NP cells expanded on ECM exhibited a huge increase in DNA and GAG amounts compared to a drop in those grown on plastic flasks despite the fact that both groups had a comparable chondrogenic index at day 14 (Figure 5.5A, 5.5B, 5.5C). After six consecutive passages, NP cells expanded on either ECM or PtoE maintained their cell numbers in pellets more than the corresponding NP cells on plastic flasks. NP cells expanded on ECM also exhibited the highest GAG amount compared to the other two groups, which contributed to the highest chondrogenic index in pellets of ECM-treated NP cells at P6 (Figure 5.5D, 5.5E, 5.5F). 
Redifferentiation capacity of expanded NP cells was also evaluated at the mRNA level (Figure 5.6). At P1, ECM-treated NP cells had a comparable collagen II but higher aggrecan mRNA than those expanded on plastic flasks after a 14-day incubation in chondrogenic medium. Although both collagen II and aggrecan mRNA levels decreased with passage, ECM-treated NP cells at P6 exhibited 10.8-fold of collagen II and 10.0-fold of aggrecan mRNA levels compared to those expanded on plastic flasks. ECM-treated NP cells also displayed a higher expression of Sox9 mRNA, which may be responsible for the enhanced expression of collagen II and aggrecan mRNAs. Our data also showed that ECM-treated NP cells at P6 exhibited about half the collagen I mRNA but comparable collagen X mRNA compared to those expanded on plastic flasks when incubated in chondrogenic medium for 14 days. 


\section{DISSCUSSION}

IVD degeneration is associated with a loss of matrix molecules resulting in altered biochemical and biomechanical properties of the tissue..$^{4 ; 16 ; 17}$ Since there is no risk of disease transmission and immune rejection, autologous IVD cells are a promising cell source for disc regeneration. However, quality and quantity of NP cells from patients are a concern. ${ }^{18}$ To make clinical application a reality, in vitro expansion cultures are expected to yield large amounts of cells in a short period of time. Moreover, these expanded NP cells should express or be able to reexpress their NP cell phonotype (redifferentiation capacity) facilitating a functional extracellular matrix. Thus, cell numbers and redifferentiation capacity are two important parameters in disc regeneration. Our study indicated, for the first time, that ECM deposited by SDSCs can provide such an in vitro microenvironment to rejuvenate NP cells by greatly enhancing cell proliferation ability, differentiation status and redifferentiation capacity.

After notochordal cells disappear during childhood, lumbar discs show evidence of degeneration with age. ${ }^{19}$ Interestingly, some species that retain their notochordal cells in adulthood do not show age-related IVD degeneration, ${ }^{20}$ suggesting that notochordal cells can provide a microenvironment preventing NP cells from degenerating. During in vitro expansion, NP cells undergo a characteristic process of dedifferentiation, marked by a loss in the expression of collagen $\mathrm{II}^{21 ; 22}$ and $\operatorname{aggrecan}^{23}$ as well as the induction of collagen I expression $^{24 ; 25}$. There is a discrepancy in the dedifferentiation process between articular chondrocytes and NP cells; the former accumulates collagen I while the later loses collagen $\mathrm{I}^{26}$. Many reports demonstrate that cells grown in a more physiological 3D environment can differ considerably in their morphology, cell-cell and cell-matrix interactions, and differentiation from those grown on flat 2D tissue culture substrates ${ }^{27 ; 28}$, suggesting that there exists a critical feature of the specialized environment (niche) in ECM that is responsible for the maintenance of MSC "stemness" by supporting their self-renewal capability and maintaining multipotentiality while facilitating differentiation in response to appropriate signals. ${ }^{29}$ However, this feature is missing in standard culture systems. 
It is postulated that ECM deposited by stem cells provides a microenvironment that can benefit adult cells in proliferation and redifferentiation capacity. Studies from our group and other groups suggest that a tissue-specific stem cell can be used to prepare its own in vitro niche for stem cell proliferation while maintaining and enhancing its lineage-specific stemness. ${ }^{13 ; 30}$ A great challenge during chondrogenesis is to generate articular cartilage-like chondrocytes that do not undergo hypertrophy as a terminal differentiation stage..$^{31-33}$ Cartilage and synovial tissue are derived from a common precursor pool ${ }^{34}$ and share similar profiles. $^{35-37}$ Recent studies demonstrate that synovial tissue can provide a superior type of MSC for chondrogenesis compared to other sources. ${ }^{38-41}$ Since NP cells are chondrocyte-like cells, SDSC-derived ECM may provide a tissue-specific microenvironment for the rejuvenation of NP cells.

In our study, NP cells grown on SDSC-derived ECM yielded greater cell numbers and higher chondrogenic marker genes than those plated on plastic flasks despite the declining trend with passage in both groups. In contrast, NP cells grown on plastic flasks resulted in cell dedifferentiation; our finding also demonstrated that these dedifferentiated cells were no longer capable of redifferentiation, especially for NP cells expanded more than four passages, which is consistent with a previous study. ${ }^{42}$ Interestingly, ECM-expanded NP cells acquired a higher redifferentiation capacity for up to six passages. Compared to being plated on plastic flasks, NP cells grown on SDSC-derived ECM regained a higher CD90 percentage and median as well as a lower CD29 median, which is consistent with our previous study in which SDSCs displayed a similar change in phenotypes when grown on SDSC-derived ECM. ${ }^{13}$ CD90 expression was reported to be positively related with MSC chondrogenic capacity ${ }^{43}$ and immunosuppressive capacity ${ }^{44}$, suggesting that ECM-treated NP cells not only acquire enhanced redifferentiation capacity but also are able to better survive the immune reaction after implantation.

There is a recent report indicating that the pathologically degenerate human disc contained populations of skeletal progenitor cells. ${ }^{45}$ These stem cells or progenitor cells would be responsible for the NP cell redifferentiation and IVD regeneration, evidenced by a higher 
proliferation capacity and multi-lineage differentiation potentials into adipogenesis, osteogenesis and chondrogenesis. ${ }^{46 ; 47}$ The nucleus pulposus cells expanded on our ECM deposited by SDSCs behaved more like stem cells or progenitor cells rather than terminally differentiated cells, suggested by the fast proliferation rate, the high expression of CD90 surface marker, and redifferentiation capacity to generate tissue-specific matrix. The specialized microenvironment is able to up-regulate a decapentaplegic (dpp) niche signaling pathway of incoming cells. Empty niches have the potential to alter cell fate as well as the growth properties of already committed and maturing cells, ${ }^{48 ; 49}$ and therefore may be able to impose a stem cell state on more mature cells, enhancing cell proliferation and differentiation potential.

Our study demonstrated that SDSC-derived ECM may provide a tissue-specific microenvironment to rejuvenate NP cells for proliferation and redifferentiation. Our study also indicated that the in vitro microenvironment from young donors may reprogram and rejuvenate seeded cells from elderly donors that have lower proliferation and differentiation capacity. ${ }^{50 ; 51}$ In clinics, most patients are adults or elders. Stem cells from older patients are not able to provide a high-quality "soil" (microenvironment) for cell rejuvenation. Stem cells from young donors in a human tissue bank could be utilized to prepare a high-quality microenvironment on which NP cells from patients can be greatly expanded while maintaining their differentiation capacity. 


\section{ACKNOWLEDGEMENTS}

We would like to thank Suzanne Smith and Mark Shoukry for editing the manuscript and Dr. Kathleen Brundage for her help with the experiments using flow cytometry, which were performed in the West Virginia University Flow Cytometry Core Facility and supported in part by NIH grant RR106440.

The manuscript submitted does not contain information about medical device(s)/drug(s). No benefits in any form have been or will be received from a commercial party related directly or indirectly to the subject of this manuscript. 


\section{REFERENCE}

1. Macfarlane GJ, Thomas E, Croft PR et al. Predictors of early improvement in low back pain amongst consulters to general practice: the influence of pre-morbid and episode-related factors. Pain 1999;80:113-9.

2. Salminen JJ, Erkintalo MO, Pentti $\mathrm{J}$ et al. Recurrent low back pain and early disc degeneration in the young. Spine (Phila Pa 1976.) 1999;24:1316-21.

3. Luoma K, Riihimaki $\mathrm{H}$, Luukkonen $\mathrm{R}$ et al. Low back pain in relation to lumbar disc degeneration. Spine (Phila Pa 1976.) 2000;25:487-92.

4. Pearce RH, Grimmer BJ, Adams ME. Degeneration and the chemical composition of the human lumbar intervertebral disc. J.Orthop.Res. 1987;5:198-205.

5. Alini $\mathrm{M}$, Roughley $\mathrm{PJ}$, Antoniou $\mathrm{J}$ et al. A biological approach to treating disc degeneration: not for today, but maybe for tomorrow. Eur.Spine J. 2002;11 Suppl 2:S215-S220.

6. An HS, Thonar EJ, Masuda K. Biological repair of intervertebral disc. Spine (Phila Pa 1976.) 2003;28:S86-S92.

7. Mwale F, Roughley P, Antoniou J. Distinction between the extracellular matrix of the nucleus pulposus and hyaline cartilage: a requisite for tissue engineering of intervertebral disc. Eur.Cell Mater. 2004;8:58-63.

8. Hohaus C, Ganey TM, Minkus $Y$ et al. Cell transplantation in lumbar spine disc degeneration disease. Eur.Spine J. 2008;17 Suppl 4:492-503.

9. Ganey $\mathrm{T}$, Libera J, Moos $\vee$ et al. Disc chondrocyte transplantation in a canine model: a treatment for degenerated or damaged intervertebral disc. Spine (Phila Pa 1976.) 2003;28:2609-20.

10. Gruber HE, Johnson TL, Leslie $\mathrm{K}$ et al. Autologous intervertebral disc cell implantation: a model using Psammomys obesus, the sand rat. Spine (Phila Pa 1976.) 2002;27:1626-33.

11. Hohaus C, Ganey TM, Minkus $Y$ et al. Cell transplantation in lumbar spine disc degeneration disease. Eur.Spine J. 2008;17 Suppl 4:492-503.

12. Evans C. Potential biologic therapies for the intervertebral disc. J.Bone Joint Surg.Am. 
2006;88 Suppl 2:95-8.

13. He F, Chen X, Pei M. Reconstruction of an in vitro tissue-specific microenvironment to rejuvenate synovium-derived stem cells for cartilage tissue engineering. Tissue Eng Part A 2009;15:3809-21.

14. Pei M, He F, Vunjak-Novakovic G. Synovium-derived stem cell-based chondrogenesis. Differentiation 2008;76:1044-56.

15. Nagase $\mathrm{T}$, Muneta $\mathrm{T}, \mathrm{Ju} \mathrm{YJ}$ et al. Analysis of the chondrogenic potential of human synovial stem cells according to harvest site and culture parameters in knees with medial compartment osteoarthritis. Arthritis Rheum. 2008;58:1389-98.

16. Buckwalter JA, Roughley PJ, Rosenberg LC. Age-related changes in cartilage proteoglycans: quantitative electron microscopic studies. Microsc.Res.Tech. 1994;28:398-408.

17. Cs-Szabo G, Ragasa-San JD, Turumella $\vee$ et al. Changes in mRNA and protein levels of proteoglycans of the anulus fibrosus and nucleus pulposus during intervertebral disc degeneration. Spine (Phila Pa 1976.) 2002;27:2212-9.

18. Singh K, Masuda K, Thonar EJ et al. Age-related changes in the extracellular matrix of nucleus pulposus and anulus fibrosus of human intervertebral disc. Spine (Phila Pa 1976.) 2009;34:10-6.

19. Miller JA, Schmatz C, Schultz AB. Lumbar disc degeneration: correlation with age, sex, and spine level in 600 autopsy specimens. Spine (Phila Pa 1976.) 1988;13:173-8.

20. Hunter CJ, Matyas JR, Duncan NA. The notochordal cell in the nucleus pulposus: a review in the context of tissue engineering. Tissue Eng 2003;9:667-77.

21. Kluba T, Niemeyer T, Gaissmaier $\mathrm{C}$ et al. Human anulus fibrosis and nucleus pulposus cells of the intervertebral disc: effect of degeneration and culture system on cell phenotype. Spine (Phila Pa 1976.) 2005;30:2743-8.

22. Tsai TT, Guttapalli A, Oguz E et al. Fibroblast growth factor-2 maintains the differentiation potential of nucleus pulposus cells in vitro: implications for cell-based transplantation therapy. Spine (Phila Pa 1976.) 2007;32:495-502.

23. Kluba T, Niemeyer $\mathrm{T}$, Gaissmaier $\mathrm{C}$ et al. Human anulus fibrosis and nucleus 
pulposus cells of the intervertebral disc: effect of degeneration and culture system on cell phenotype. Spine (Phila Pa 1976.) 2005;30:2743-8.

24. Kluba T, Niemeyer $T$, Gaissmaier $C$ et al. Human anulus fibrosis and nucleus pulposus cells of the intervertebral disc: effect of degeneration and culture system on cell phenotype. Spine (Phila Pa 1976.) 2005;30:2743-8.

25. Tsai TT, Guttapalli A, Oguz E et al. Fibroblast growth factor-2 maintains the differentiation potential of nucleus pulposus cells in vitro: implications for cell-based transplantation therapy. Spine (Phila Pa 1976.) 2007;32:495-502.

26. Tsai TT, Guttapalli A, Oguz E et al. Fibroblast growth factor-2 maintains the differentiation potential of nucleus pulposus cells in vitro: implications for cell-based transplantation therapy. Spine (Phila Pa 1976.) 2007;32:495-502.

27. Cukierman E, Pankov R, Yamada KM. Cell interactions with three-dimensional matrices. Curr.Opin. Cell Biol. 2002;14:633-9.

28. Griffith LG, Swartz MA. Capturing complex 3D tissue physiology in vitro. Nat.Rev.Mol.Cell Biol. 2006;7:211-24.

29. Moore KA, Lemischka IR. Stem cells and their niches. Science 2006;311:1880-5.

30. Chen XD, Dusevich $\mathrm{V}$, Feng JQ et al. Extracellular matrix made by bone marrow cells facilitates expansion of marrow-derived mesenchymal progenitor cells and prevents their differentiation into osteoblasts. J.Bone Miner.Res. 2007;22:1943-56.

31. De BC, Dell'Accio F, Karystinou A et al. A biomarker-based mathematical model to predict bone-forming potency of human synovial and periosteal mesenchymal stem cells. Arthritis Rheum. 2008;58:240-50.

32. Muraglia A, Cancedda R, Quarto R. Clonal mesenchymal progenitors from human bone marrow differentiate in vitro according to a hierarchical model. J.Cell Sci. 2000;113 ( Pt 7):1161-6.

33. Pelttari K, Winter A, Steck $\mathrm{E}$ et al. Premature induction of hypertrophy during in vitro chondrogenesis of human mesenchymal stem cells correlates with calcification and vascular invasion after ectopic transplantation in SCID mice. Arthritis Rheum. 2006;54:3254-66.

34. Pacifici M, Koyama E, Iwamoto M et al. Development of articular cartilage: what do we 
know about it and how may it occur? Connect.Tissue Res. 2000;41:175-84.

35. Fife RS, Caterson B, Myers SL. Identification of link proteins in canine synovial cell cultures and canine articular cartilage. J.Cell Biol. 1985;100:1050-5.

36. Hamerman D, Smith C, Keiser HD et al. Glycosaminoglycans produced by human synovial cell cultures. Coll.Relat Res. 1982;2:313-29.

37. Recklies $A D$, Baillargeon $L$, White $C$. Regulation of cartilage oligomeric matrix protein synthesis in human synovial cells and articular chondrocytes. Arthritis Rheum. 1998;41:997-1006.

38. Mochizuki T, Muneta T, Sakaguchi $Y$ et al. Higher chondrogenic potential of fibrous synovium- and adipose synovium-derived cells compared with subcutaneous fat-derived cells: distinguishing properties of mesenchymal stem cells in humans. Arthritis Rheum. 2006;54:843-53.

39. Sakaguchi $\mathrm{Y}$, Sekiya I, Yagishita $\mathrm{K}$ et al. Comparison of human stem cells derived from various mesenchymal tissues: superiority of synovium as a cell source. Arthritis Rheum. 2005;52:2521-9.

40. Shirasawa S, Sekiya I, Sakaguchi $Y$ et al. In vitro chondrogenesis of human synovium-derived mesenchymal stem cells: optimal condition and comparison with bone marrow-derived cells. J. Cell Biochem. 2006;97:84-97.

41. Yoshimura $H$, Muneta $T$, Nimura $A$ et al. Comparison of rat mesenchymal stem cells derived from bone marrow, synovium, periosteum, adipose tissue, and muscle. Cell Tissue Res. 2007;327:449-62.

42. Schulze-Tanzil G, de SP, Villegas $\mathrm{CH}$ et al. Redifferentiation of dedifferentiated human chondrocytes in high-density cultures. Cell Tissue Res. 2002;308:371-9.

43. Nagase $\mathrm{T}$, Muneta $\mathrm{T}, \mathrm{Ju} \mathrm{YJ}$ et al. Analysis of the chondrogenic potential of human synovial stem cells according to harvest site and culture parameters in knees with medial compartment osteoarthritis. Arthritis Rheum. 2008;58:1389-98.

44. Campioni D, Rizzo R, Stignani M et al. A decreased positivity for CD90 on human mesenchymal stromal cells (MSCs) is associated with a loss of immunosuppressive activity by MSCs. Cytometry B Clin. Cytom. 2009;76:225-30.

45. Risbud MV, Guttapalli A, Tsai TT et al. Evidence for skeletal progenitor cells in the 
degenerate human intervertebral disc. Spine (Phila Pa 1976.) 2007;32:2537-44.

46. Risbud MV, Guttapalli A, Tsai TT et al. Evidence for skeletal progenitor cells in the degenerate human intervertebral disc. Spine (Phila Pa 1976.) 2007;32:2537-44.

47. Henriksson $\mathrm{H}$, Thornemo $\mathrm{M}$, Karlsson $\mathrm{C}$ et al. Identification of cell proliferation zones, progenitor cells and a potential stem cell niche in the intervertebral disc region: a study in four species. Spine (Phila Pa 1976.) 2009;34:2278-87.

48. Kai T, Spradling A. An empty Drosophila stem cell niche reactivates the proliferation of ectopic cells. Proc.NatI.Acad.Sci.U.S.A 2003;100:4633-8.

49. Kai T, Spradling A. Differentiating germ cells can revert into functional stem cells in Drosophila melanogaster ovaries. Nature 2004;428:564-9.

50. Barbero A, Grogan S, Schafer D et al. Age related changes in human articular chondrocyte yield, proliferation and post-expansion chondrogenic capacity. Osteoarthritis. Cartilage. 2004;12:476-84.

51. Conboy IM, Conboy MJ, Wagers AJ et al. Rejuvenation of aged progenitor cells by exposure to a young systemic environment. Nature 2005;433:760-4. 


\section{FIGURE LEGENDS}

Figure 5.1. Effect of SDSC-derived ECM on NP cell morphology and proliferation. NP cells were expanded on either plastic flasks or ECM for six consecutive passages. NP cell morphology at P1 and P4 was shown at day 6 using a phase contrast microscope (A). Cell number from both groups was compared with passage (B). Data are shown as average \pm SD for $n=3$.

Figure 5.2. Flow cytometry analysis of typical MSC surface antigens (CD29, CD44, and CD90) with isotype-specific control (A). The percentage (B, D, F) and mean fluorescent intensity (MFI, C, E, G) of positive cells were compared for MSC phenotype changes in NP cells grown on plastic flasks or ECM for six passages.

Figure 5.3. Quantitative real-time PCR of differentiated (A: collagen II mRNA, B: aggrecan mRNA, and C: collagen X mRNA) and dedifferentiated (D: collagen I mRNA) phenotypes of NP cells expanded on plastic flasks or ECM for six passages. The data were adjusted by mRNA level of P1 NP cells expanded on plastic flasks. Data are shown as average \pm SD for $n$ $=5$.

Figure 5.4. Histological evaluation of redifferentiation capacity of NP cells expanded on plastic flasks or ECM for six passages using safranin $O$ staining for sulfated GAGs and immunostaining for types I and II collagen at days 0,7 , and 14. NP cells grown on plastic flasks for five passages and switched to ECM for one passage (PtoE) served as the third group. The scale bar is $800 \mu \mathrm{m}$.

Figure 5.5. Biochemical analyses were used to detect DNA $(A, D)$ and $G A G$ amounts $(B, E)$ per pellet at $P 1(A, B, C)$ and $P 6(D, E, F)$. DNA amount at days 7 and 14 was adjusted by that at day 0 to minimize the initial difference in pellet preparation. Chondrogenic index was presented as ratio of GAG to DNA at P1 (C) and P6 (F). Data are shown as average \pm SD for $\mathrm{n}=6$. Significant differences are indicated as ${ }^{*} p<0.05,{ }^{* *} p<0.01$ and ${ }^{* *} p<0.001$. 
Figure 5.6. $\operatorname{TaqMan}^{\circledR}$ real-time PCR was used to quantitatively assess chondrogenic markers [collagen II (A/B), aggrecan (C/D), Sox9 (E/F), collagen I (G/H), and collagen X (I/J)] at mRNA level. All marker gene expression was presented as relative mRNA level adjusted with $18 \mathrm{~S}$ RNA. Data are shown as average \pm SD for $n=5$. Significant differences are indicated as ${ }^{*} p<$ $0.05,{ }^{* *} p<0.01$ and ${ }^{* *} p<0.001$. 
Figure 5.1

A

Passage 1

Passage 4
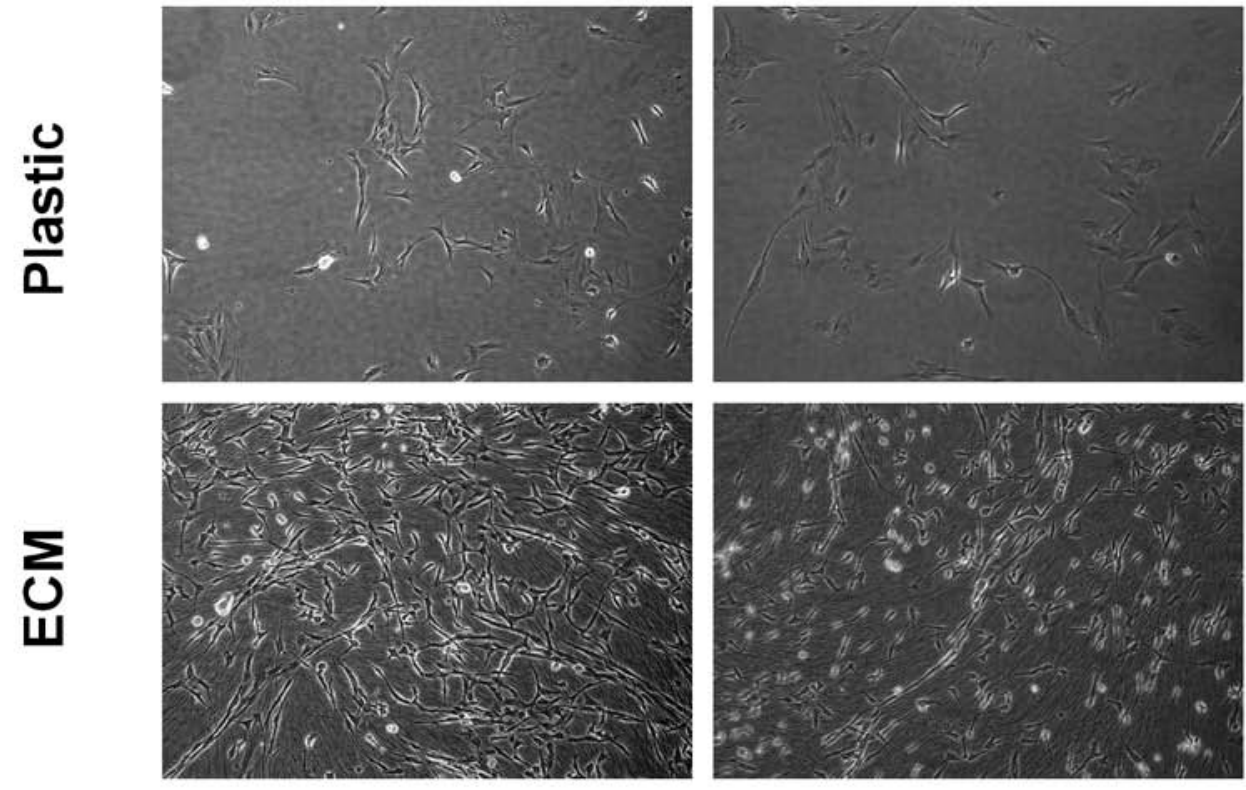

B

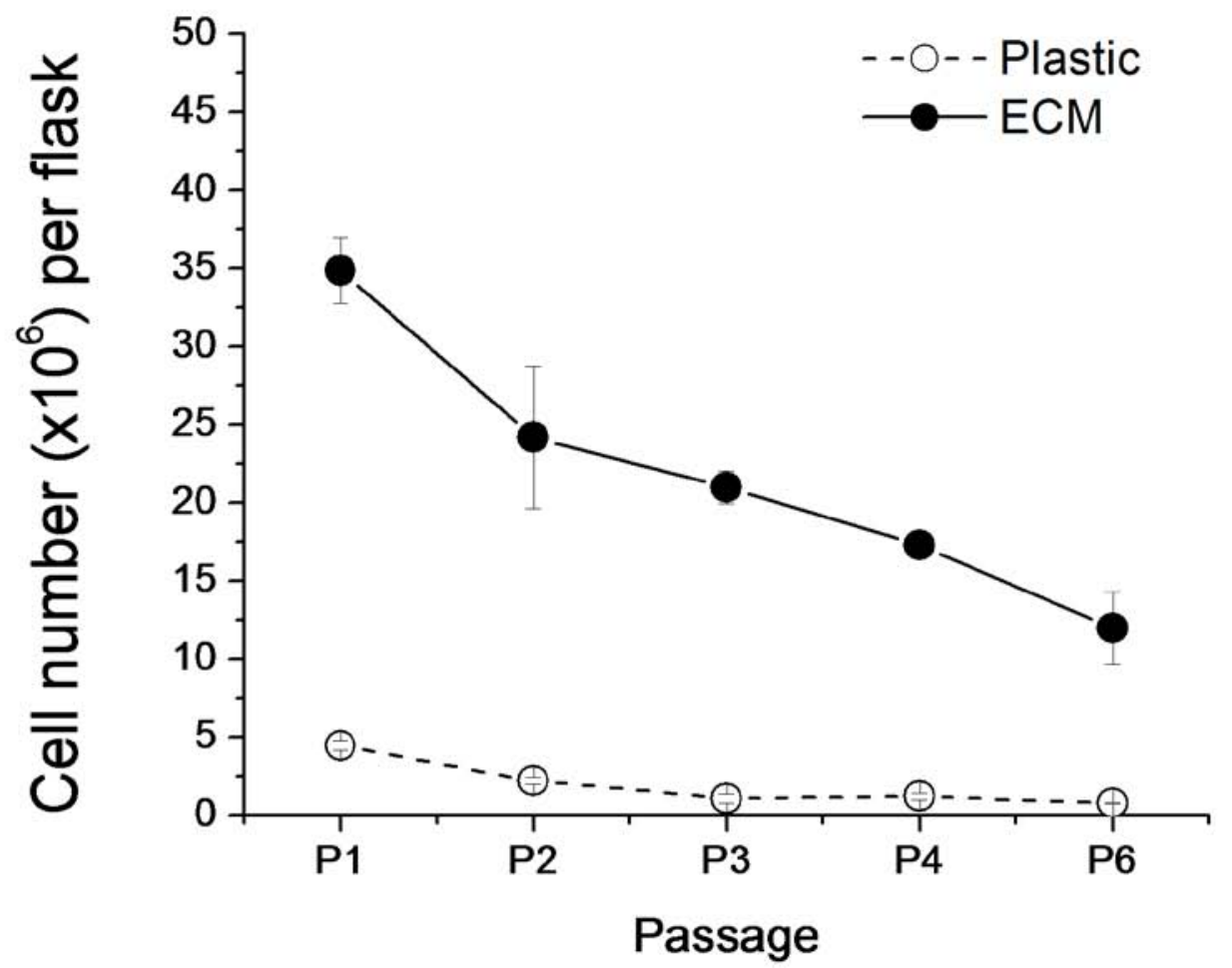


Figure 5.2

A

Passage 1

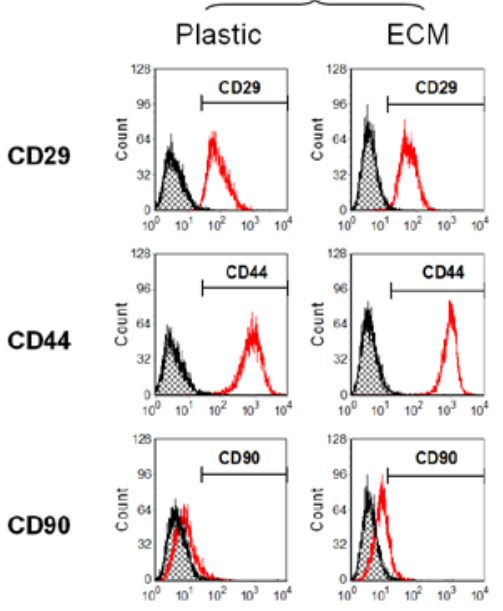

Passage 4
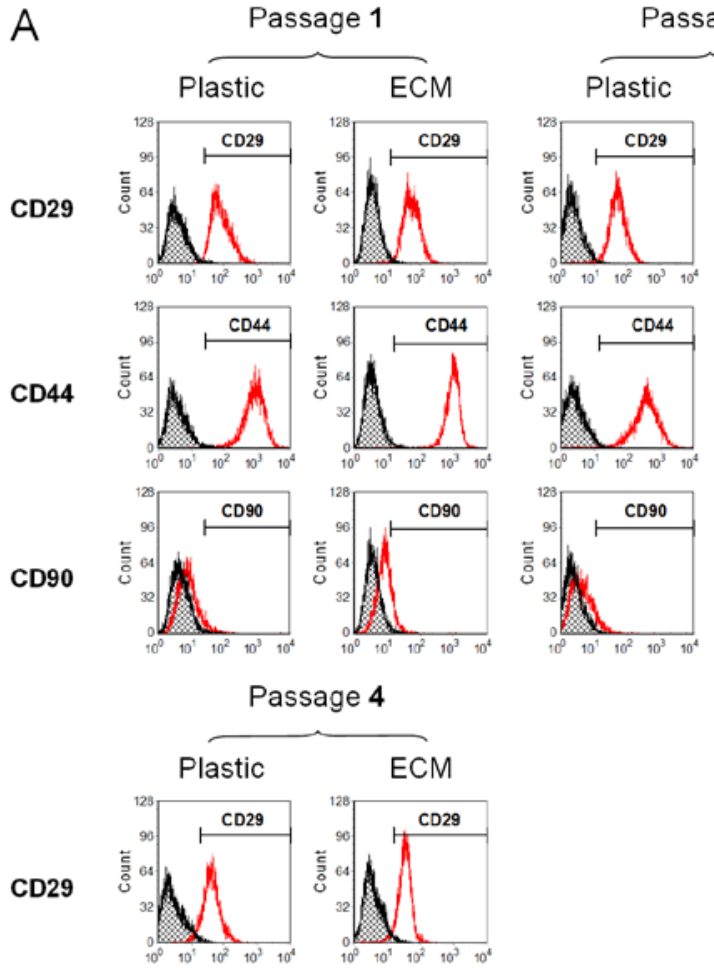

assage 2
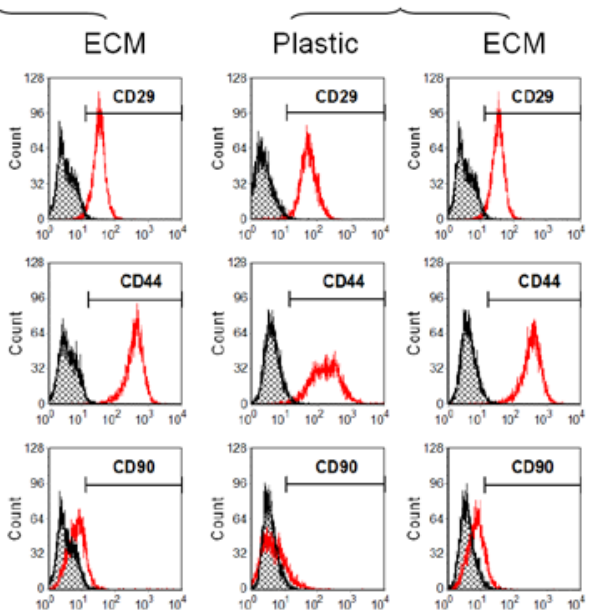

Passage 6
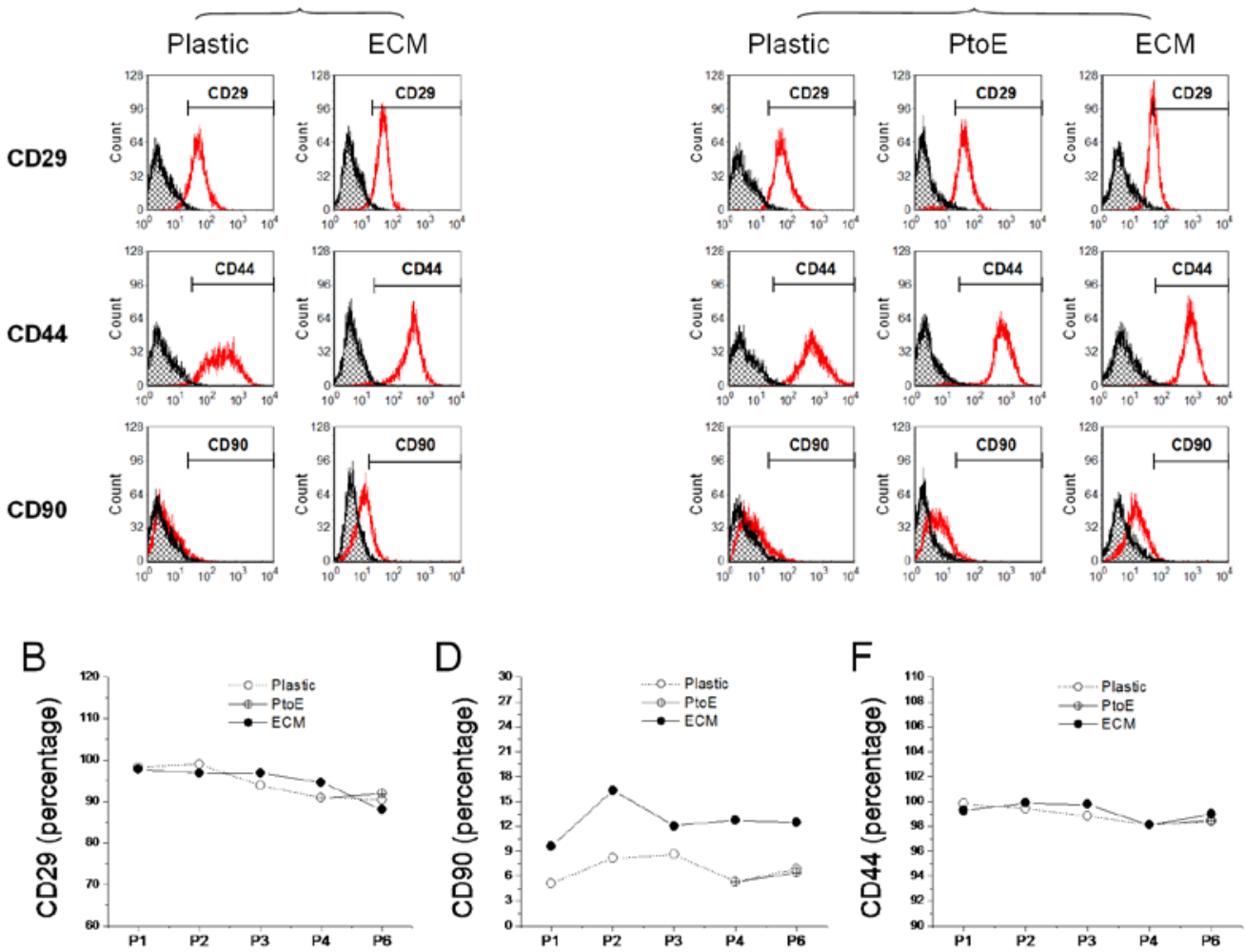

C

E
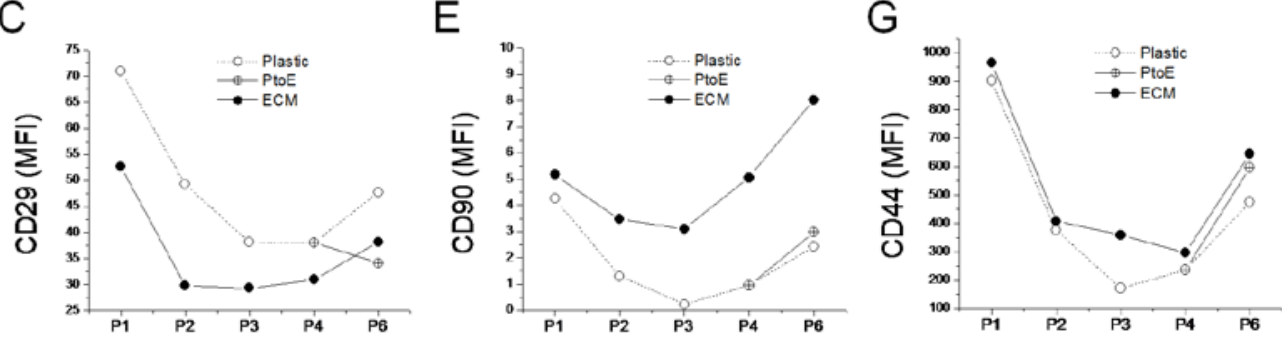
Figure 5.3
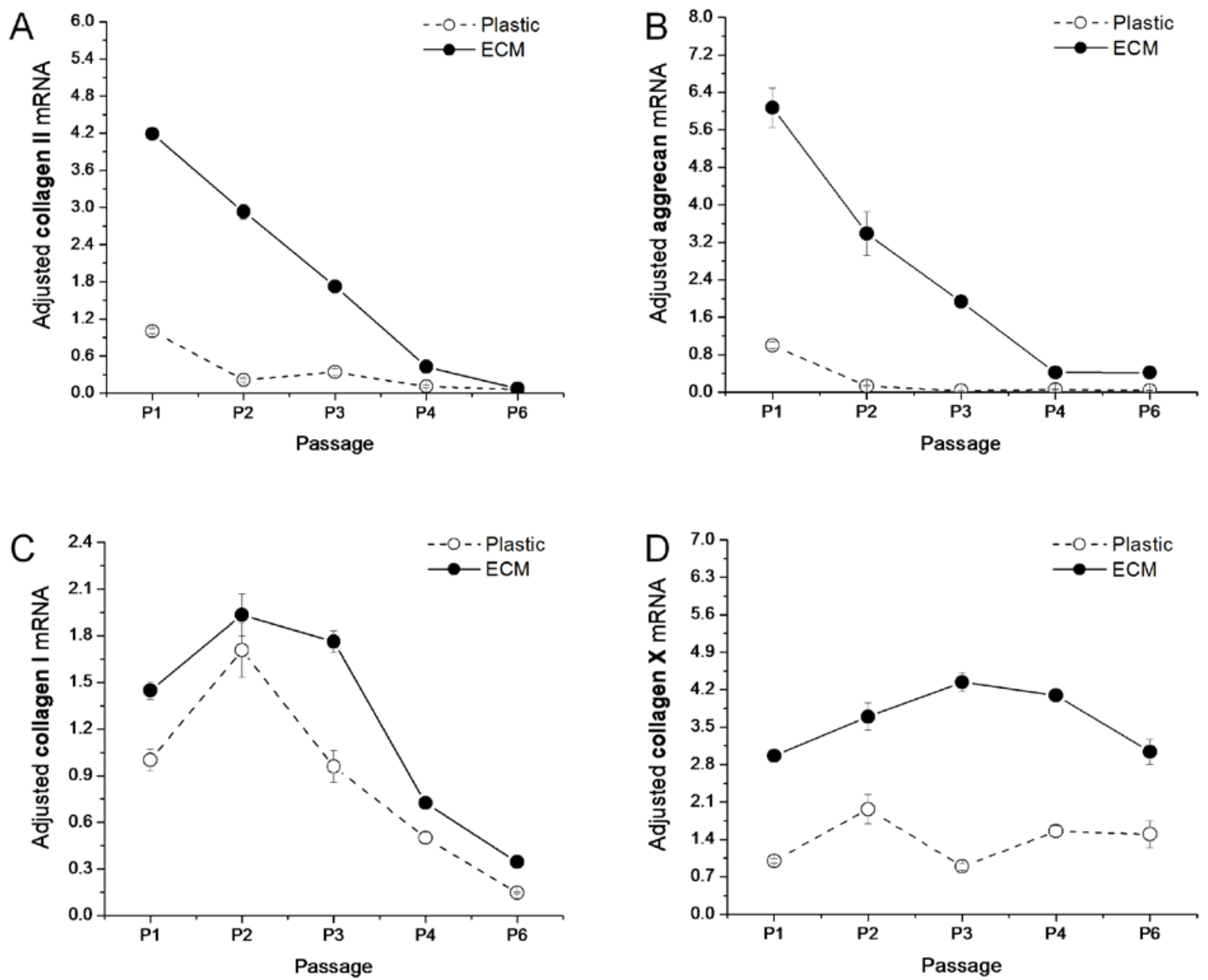
Figure 5.4

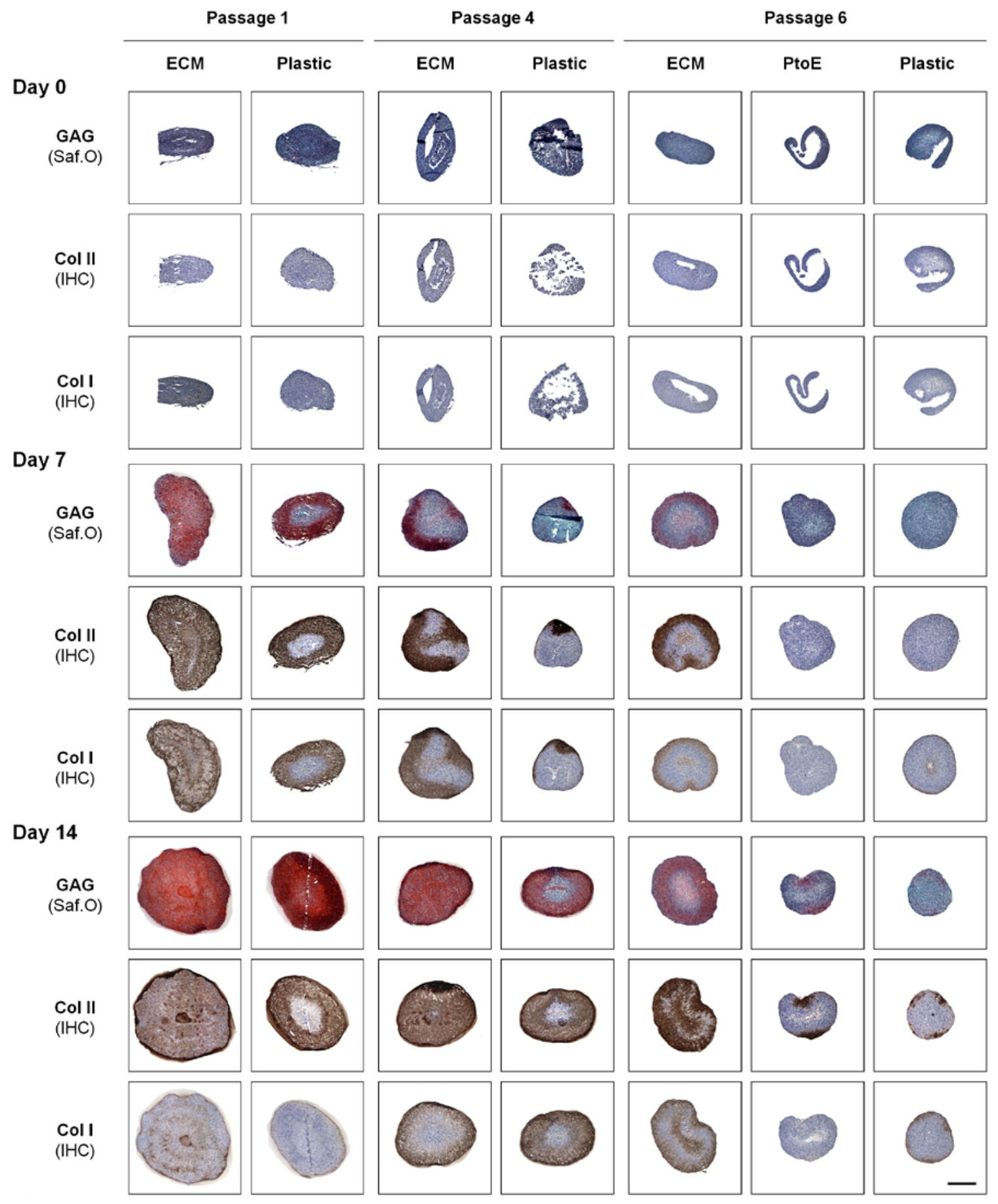


Figure 5.5
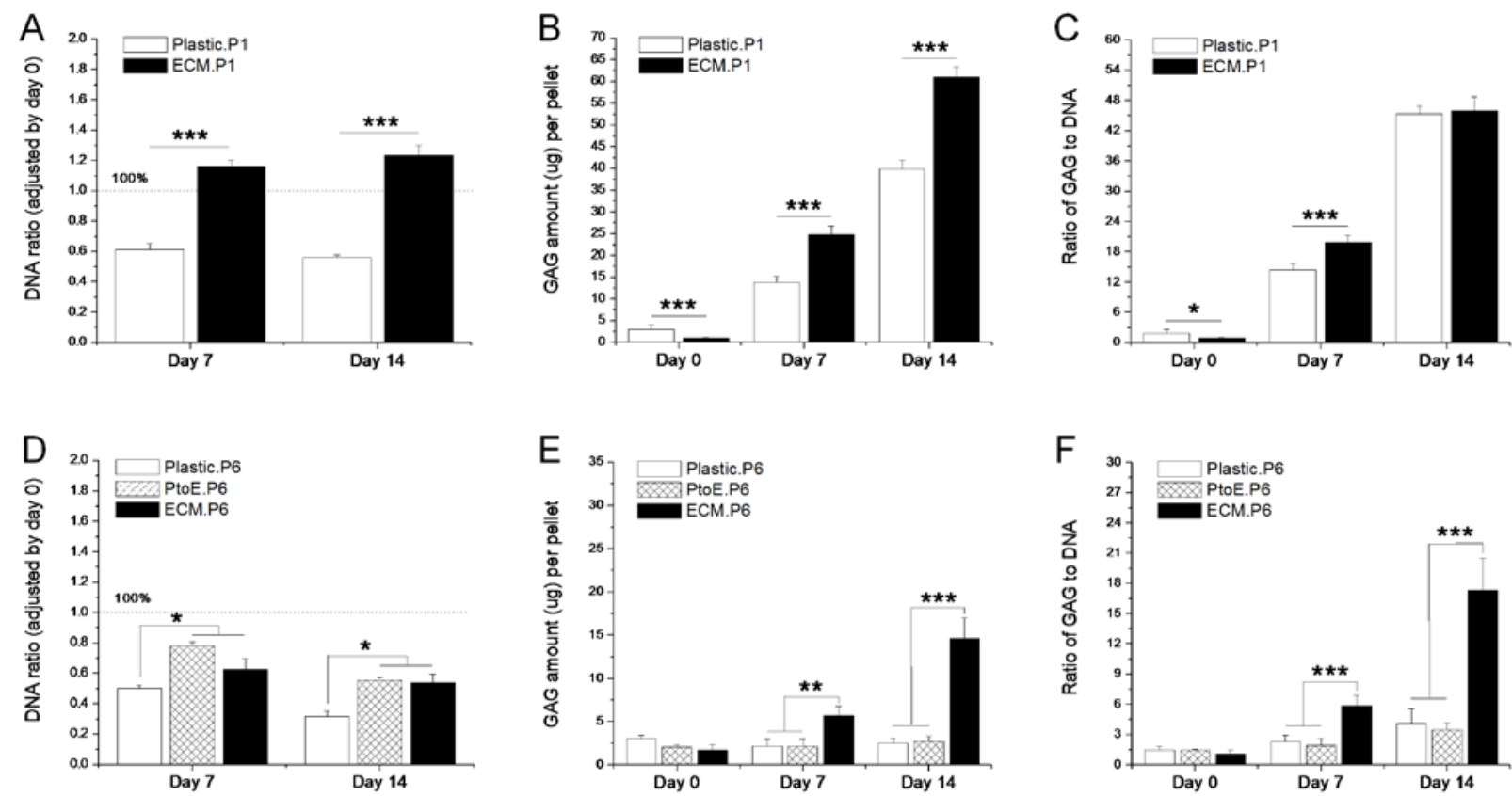
Figure 5.6
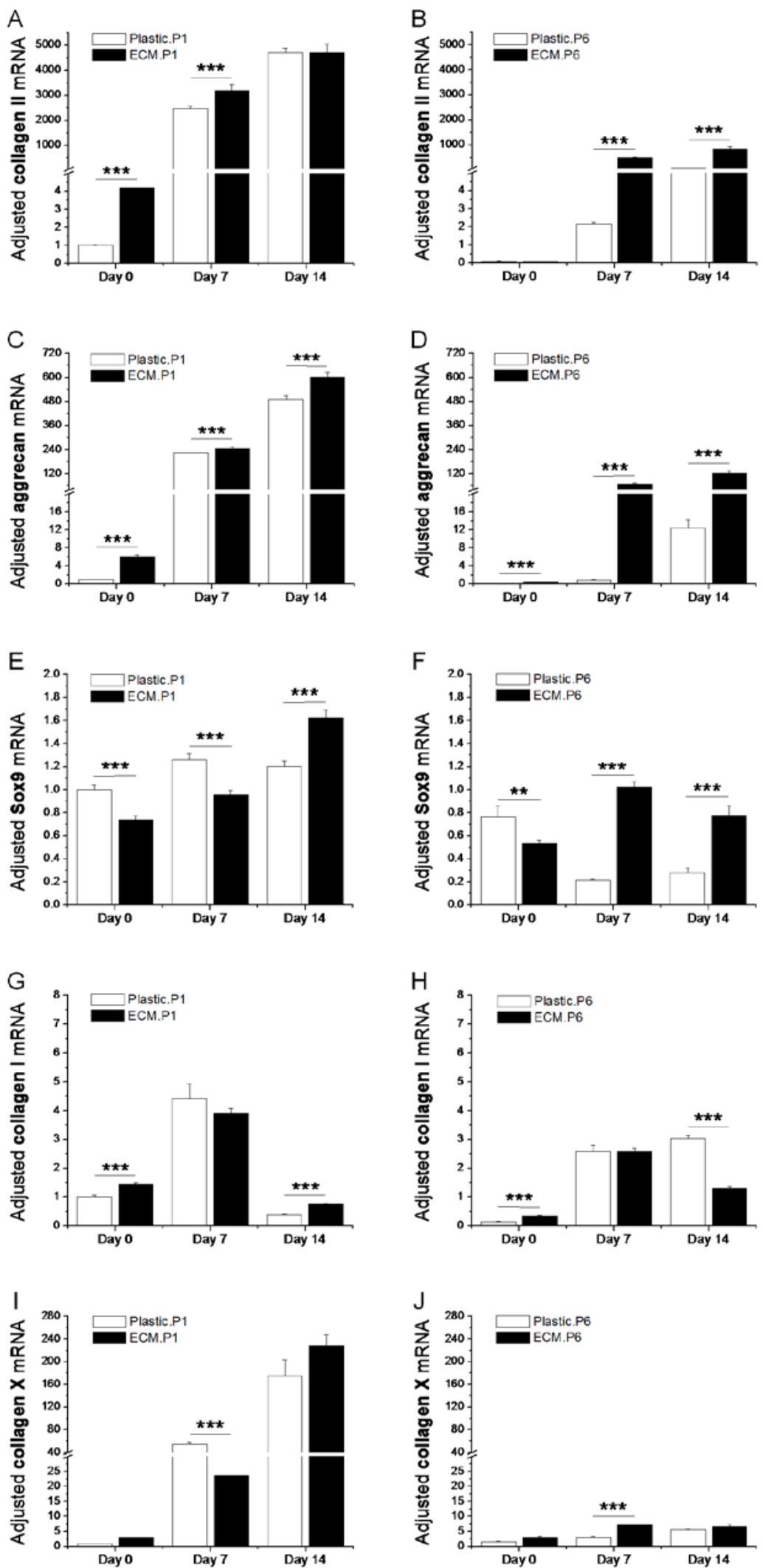


\section{Chapter 6}

Three-dimensional extracellular matrix deposited by human bone marrow stem cells promotes cell proliferation and chondrogenesis

ECM interacts with cells via integrin/Src/ERK signaling pathway and enhances the sensitivity of transforming growth factor beta receptor II

Fan He, B.S. and Ming Pei, M.D., Ph.D.

Stem Cell and Tissue Engineering Laboratory, Department of Orthopaedics and Division of Exercise Physiology, West Virginia University, Morgantown, West Virginia 26506

Key words: Bone marrow stem cells, Extracellular matrix, Chondrogenesis, Cyclin D1, ERK 


\begin{abstract}
The three-dimensional (3D) cell-deposited extracellular matrix (ECM) was previously reported as a new expansion system in vitro mimicking tissue-specific mesenchymal stem cell microenvironment, but the mechanism of the interaction between ECM and cells and the intracellular signaling transduction pathway was not clear due to the complexity of the 3D ECM compared with the 2D conventional monolayer culture. In this study we expanded human bone marrow stem cells (hBMSCs) in vitro under the 3D hBMSC-deposited ECM environment (ECM) or conventional plastic flasks (Plastic). The 3D ECM greatly improved hBMSC proliferative rate and attenuated the intracellular reactive oxygen species but increased the cell apoptosis. Chondrogenic differentiation was induced by transforming growth factor - $\beta 3$ (TGF- $\beta 3$ ) and hBMSCs expanded on 3D ECM showed robust chondrogenic potential to generate cartilage-specific genes and matrix, including sulfated glycosaminoglycans, Sox9 and type II collagen. We compared several key signaling kinases expression and activity in hBMSCs cultured on ECM and Plastic. The 3D ECM increased the expression of SSEA-4, integrin $\alpha_{2}, \alpha_{4}$, and $\beta_{5}$, and induced sustained activation of Src kinase, ERK1/2 and cyclin D1. Not only the expression but also the activity of TGF- $\beta$ receptor II was enhanced by 3D ECM in hBMSCs. These observations suggested that the 3D cell-deposited ECM promoted hBMSC proliferative capacity may through integrin/Src/ERK1/2 signaling pathway and enhanced the chondrogenic potential by improving the responsiveness of TGF- $\beta$ receptor.
\end{abstract}




\section{INTRODUCTION}

Mesenchymal stem cells (MSCs) are considered a promising source for cell therapy and regenerative medicine, because not only they can be isolated from various adult tissues, but also they have excellent self-renewal potential and multipotent differentiation capacities. ${ }^{1-4}$ The current expansion technique in vitro, however, limits the utilization of MSCs due to inadequate cell quantity and impaired multipotent differentiation after monolayer passages. ${ }^{5 ; 6}$ In previous studies, we developed a novel mesenchymal stem cells expansion system based on the three-dimensional (3D) extracellular matrix (ECM) deposited by synovium-derived stem cells (SDSCS). This system dramatically improved the proliferation and chondrogenic potential of SDSCs ${ }^{7}$ and adipose-derived stem cells (ADSCs).

These natural ECM were first used to investigate tumor progression ${ }^{8 ; 9}$ and provided an excellent model to study cell behaviors in the $3 D$ environment. The native $3 D$ ECM is proposed to mimic the in vivo microenvironment, thus directing MSCs in morphology, cell-cell and cell-matrix interaction, self-renewal and differentiation. ${ }^{\text {7:10;11 }}$ Compared to conventional 2D reconstructed ECM, which is usually coated with several protein monolayers, including fibronectin, ${ }^{12}$ type I collagen ${ }^{13}$ or laminins, ${ }^{14}$ the native cell-deposited ECM not only provides 3D structure but also has multiply matrix proteins to affect the cell signaling pathway, including type I, III and V collagens, syndecan-1, perlecan, fibronectin, laminin, biglycan and decorin. $^{7 ; 10 ; 15}$

Integrins are heterodimeric transmembrane receptors that are composed by an $\alpha$ and a $\beta$ subunit. They mediate the interaction between ECM and cells, thus affecting the cell adhesion, survival, self-renewal, apoptosis, migration and differentiation ${ }^{16}$. Attaching to various ECM proteins, integrins combine two different $\alpha$ and $\beta$ subunits. $\beta 1$ integrin are proven to bind collagen and fibronectin ${ }^{17}$ and $\alpha_{5} \beta_{1}$ and $\alpha_{v} \beta_{3}$ integrins were reported in the process of cells attaching on the 3D ECM. ${ }^{15 ; 18} \mathrm{ECM}$ provides a structural scaffold to resist tensile and compressive stress and functions as a tight connection to cell cytoskeleton through cell-surface receptors enabling cells to sense and respond to the extracellular 
mechanical and chemical signals. ${ }^{16 ; 19 ; 20}$

However, the cell signaling pathway associated with 3D native ECM is still unclear and different from that on $2 \mathrm{D}$ reconstructed $\mathrm{ECM}$. It is reported that the activity of focal adhesion kinase (FAK) which mediates integrin activation is suppressed by 3D ECM. But ECM induced sustained phosphorylation of Src kinase, whose downstream extracellular signal regulated kinases 1 and $2(E R K 1 / 2)$ thus is elevated. ${ }^{11 ; 15 ; 21}$ Integrins also play an important role in cell proliferation through ERK1/2 dependant pathway by activating cyclin D1 to promote cell division. ${ }^{19 ; 22 ; 23}$ Several integrins, including $\alpha_{5} \beta_{1}, \alpha_{v} \beta_{3}$ and $\alpha_{4} \beta_{1}$ were reported to be activated when binding to ECM components such as fibronectin and collagens. ${ }^{16 ; 20 ; 24-27}$

In this study, we expanded human bone marrow stem cells (hBMSCs) under two different conditions: (1) cells grown on conventional plastic flasks were used as the control group (Plastic); (2) cells were cultured on 3D native hBMSC-deposited ECM (ECM). Considering the importance of integrins and ERK1/2 in mediating cell proliferation, we tested 10 integrin subunits immunophenotype, and the activation level of Src kinase, ERK1/2 and cyclin D1. After separate expansion on ECM and Plastic, hBMSCs were induced to chondrogenesis and several key proteins were examined including transforming growth factor $\beta$ receptor II (TGF- $\beta$ receptor II), Sox9, type II collagen and ERK1/2. Interestingly, we found that 3D hBMSC-deposited ECM induced sustained activation of ERK1/2 and cyclin D1 and enhanced TGF $\beta$ receptor II activity during chondrogenesis. 


\section{MATERIALS AND METHODS}

\section{Preparation of hBMSC-derived extracellular matrix (ECM)}

Human bone marrow stem cells (hBMSCs) were purchased from Lonza (Lonza Group Ltd., Switzerland). The procedure of obtaining cell-free extracellular matrix was described previously ${ }^{7} .75 \mathrm{~cm}^{2}$ plastic flasks were pretreated with $0.2 \%$ gelatin solution (Sigma, St. Louis, $\mathrm{MO}$ ) for 1 hour at 36 , followed by $1 \%$ glutaraldehyde (Sigma) and $1 \mathrm{M}$ ethanolamine (Sigma) for 30 minutes separately at room temperature. Human BMSCs were seeded on pretreated plastic flasks at the density of $3,000 \mathrm{cells} / \mathrm{cm}^{2}$ in culture medium $[\alpha-$ minimum essential medium (Invitrogen, Carlsbad, CA) containing 10\% fetal bovine serum (FBS, Atlanta Biologicals, Lawrenceville, GA), $100 \mathrm{U} / \mathrm{ml}$ penicillin, $100 \mu \mathrm{g} / \mathrm{ml}$ streptomycin and 0.25 $\mu \mathrm{g} / \mathrm{ml}$ fungizone (Invitrogen)] for 7 days. After reaching $90 \%$ confluence, $50 \mathrm{mg} / \mathrm{mL}$ of L-ascorbic acid phosphate (Wako, Richmond, VA) was added and continued to culture for an additional 8 days. To obtain cell-free ECM, ECM deposited by hBMSCs was incubated with 0.5\% Triton X-100 (Sigma) containing 20 mM ammonium hydroxide (Sargent-Welch, Skokie, IL) for 5 minutes at $37^{\circ} \mathrm{C}$, followed by $100 \mathrm{u} / \mathrm{mL}$ DNase I (Sigma) for 1 hour åC 3 and $^{2}$ stored at $4^{\circ} \mathrm{C}$.

\section{In vitro culture of hBMSCS on Plastic and ECM}

Passage 6 BMSCs were expanded under two different conditions: conventional plastic flasks (Plastic) and flasks coated with hBMSC-derived ECM (ECM). Non-adherent cells were removed by medium change every three days. At each passage, cell number was calculated by a counting hemacytometer (Hausser Scientific, Horsham, PA) for cell number fold change.

\section{Cell proliferation assay}

Before subculturing on Plastic and ECM, passage 6 hBMSCs were labeled with CellVue ${ }^{\circledR}$ Claret (Sigma) at $2 \times 10^{-6} \mathrm{M}$ for 5 minutes according to the manufacture protocol. After the 7-day expansion, cells were collected and measured by a BD dual laser FACS Calibur (BD Biosciences). Cell proliferation index was analyzed using CellQuest Pro software (BD Biosciences). 
Measurement of intracellular reactive oxygen species (ROS)

Intracellular ROS generation was measured with 2',7'-dichlorofluorescein diacetate (DCF-DA) (Sigma). In brief, after detachment, $2 \times 10^{5}$ of cells $(n=3)$ were incubated with $10 \mu \mathrm{M}$ of DCF-DA for 10 minutes at $37^{\circ} \mathrm{C}$. After washing twice with PBS, the DCF fluorescence was measured by a BD dual laser FACS Calibur (BD Biosciences) using FCS Express software package (De Novo Software, Los Angeles, CA) and 10000 events of each sample were analyzed.

\section{Apoptosis analysis of hBMSCs on Plastic and ECM}

Vybrant $^{\circledR}$ Apoptosis Assay Kit (Invitrogen) was used to detect hBMSCs apoptosis. By following the manual, samples $(n=3)$ of each $2 \times 10^{5}$ cells were labeled with FITC annexin $V$ and propidium iodide for 15 minutes at room temperature. After washing with PBS the fluorescence was measured by FACS Calibur (BD Biosciences) and 10000 events of each sample were analyzed.

\section{Flow cytometry analysis}

The following primary antibodies were used in flow cytometry analysis to detect hBMSCs surface immunophenotype profiles: CD14, CD24, integrin $\beta_{1}$ (CD29), CD44, CD45, CD71, CD73, CD166 were purchased from Abcam (Abcam, Cambridge, MA); CD105, SSEA-4, integrins of $\alpha_{1}$ (CD49a), $\alpha_{2}$ (CD49b), $\alpha_{3}$ (CD49c), $\alpha_{4}$ (CD49d), $\alpha_{5}$ (CD49e), $\alpha_{6}$ (CD49f), $\beta_{3}$ (CD61), and $\beta_{4}$ (CD104) were from Santa Cruz (Santa Cruz Biotechnology, Santa Cruz, CA); CD90 (BD Biosciences); Integrin $\beta_{5}$ (Cell Signaling Technology, Danvers, MA). IgG1 and IgG2a (Beckman Coulter, Fullerton, CA) were used as the isotype controls, and the secondary antibody was goat anti-mouse IgG $(\mathrm{H}+\mathrm{L})$ R-phycoerythrin conjugated (Invitrogen). Samples $(n=3)$ of each $0.2 \times 10^{6}$ chondrocytes were incubated on ice in cold PBS containing 0.1\% ChromPure Human IgG whole molecule (Jackson ImmunoResearch Laboratories, West Grove, PA) and $1 \% \mathrm{NaN}_{3}$ (Sigma) for 30 minutes. After that cells were incubated in the primary antibodies and the secondary antibodies for 30 minutes separately in the dark. The fluorescence was analyzed by a FACS Calibur (BD Biosciences) using FCS Express software 
package (De Novo Software).

\section{Chondrogenic differentiation of hBMSCs}

$3 \times 10^{5}$ of hBMSCs at passage 6 cultured on Plastic and ECM were centrifuged to form pellets in a 15-ml polypropylene tube at $500 \mathrm{~g}$ for 5 minutes. After 24 hours the pellets were incubated in chondrogenic medium [high-glucose DMEM, $40 \mu \mathrm{g} / \mathrm{ml}$ proline, $100 \mathrm{nM}$ dexamethasone (Sigma), $100 \mathrm{U} / \mathrm{ml}$ penicillin, $100 \mu \mathrm{g} / \mathrm{ml}$ streptomycin, $0.1 \mathrm{mM}$ ascorbic acid-2-phosphate and ITS ${ }^{\mathrm{TM}}$ Premix (BD Biosciences) with the supplementation of $10 \mathrm{ng} / \mathrm{ml}$ of TGF- $\beta 3$ (PeproTech Inc., Rocky Hill, NJ)]. Pellets at day 0, day 7 and day 15 were collected for further analysis.

\section{Histology and Immunohistochemistry}

The chondrogenic pellets $(n=2)$ were fixed in $4 \%$ paraformaldehyde, embedded in paraffin blocks and cut into 5- $\mu \mathrm{m}$ thick sections. To detect glycosaminoglycans, sections were stained with $0.2 \%$ Alcian blue solution (Sigma). For immunohistochemistry the sections were incubated with primary antibodies against type II collagen (II-II6B3, DSHB, Iowa City, IA), type I collagen (Sigma) and type $X$ collagen (Sigma), followed by the secondary antibody of biotinylated horse anti-mouse IgG (Vector, Burlingame, CA) and detected by using Vectastain $A B C$ reagent (Vector) with 3,3'-diaminobenzidine (DAB) as the substrate.

\section{Biomechanical analysis for hBMSC pellets}

The pellets $(n=4)$ were placed into a reservoir filled with PBS and loaded onto the custom miniature stepper motor driven compression device. This device used a $10 \mathrm{gram}$ load and a miniature displacement readout. A small preload of $10^{-4}$ Newton was applied before a 10 percent strain at a 1 second duration. From the linear portion of the load displacement curve, the stiffness and Young's modulus were calculated for the spherical shaped pellet. ${ }^{28}$

\section{Biochemical analysis for DNA and glycosaminoglycan content}

To exam DNA and sulfated glycosaminoglycans (GAGs) content, pellets $(n=4)$ were digested for 6 hours at $60^{\circ} \mathrm{C}$ with $125 \mu \mathrm{g} / \mathrm{ml}$ papain in PBE buffer (100 mM phosphate, $10 \mathrm{mM}$ 
EDTA, pH 6.5) containing $10 \mathrm{mmol} / \mathrm{L}$ cysteine. The amount of DNA was measured by $\mathrm{e}$ QuantiT $^{\mathrm{TM}}$ PicoGreen $^{\circledR}$ dsDNA assay kit (Invitrogen) with a CytoFluor ${ }^{\circledR}$ Series 4000 (Applied Biosystems, Foster City, CA) followed by the protocol. Sulfated GAGs were measured by using $16 \mu \mathrm{g} / \mathrm{ml}$ of dimethylmethylene blue dye and a Spectronic $^{\mathrm{TM}}$ BioMate $^{\mathrm{TM}} 3$ Spectrophotometer (Thermo Scientific, Milford, MA) with bovine chondroitin sulfate (Sigma) as the standard.

\section{Real-time polymerase chain reaction (Real-time PCR)}

The total mRNA from pellets $(n=4)$ was extracted by using TRIzol ${ }^{\circledR}$ (Invitrogen) and converted to cDNA with High-Capacity cDNA Archive Kit as recommended by the manufacturer (Applied Biosystems). Chondrogenic marker genes (aggrecan, type I and II collagen, and Sox9) and hypertrophy marker genes (type $X$ collagen, MMP13) are customized by Applied Biosystems as part of the Custom Taqman ${ }^{\circledR}$ Gene. Expression Assays Eukaryotic 18S rRNA (Assay ID HS99999901_s1 ABI) was carried out as the endogenous control gene. Real-time PCR was performed with the iCycler $\mathrm{iQ}^{\mathrm{TM}}$ Multi Color RT-PCR Detection and calculated by computer software (Perkin-Elmer, Waltham, MA). Relative transcript levels were calculated as $\mathrm{X}=2^{-\Delta \Delta \mathrm{Ct}}$, in which $\Delta \Delta \mathrm{Ct}=\Delta \mathrm{E}-\Delta \mathrm{C}, \Delta \mathrm{E}=\mathrm{Ct}_{\mathrm{exp}}-\mathrm{Ct}_{18 \mathrm{~s}}$, and $\Delta \mathrm{C}=\mathrm{Ct}_{\mathrm{ct1}}-\mathrm{Ct}_{18 \mathrm{~s}}$.

\section{Immunoblotting}

RIPA lysis buffer (Thermo Scientific) supplemented with halt protease and phosphatase inhibitor cocktail (Thermo Scientific) was used to extract protein from the cells. The protein concentration was quantified using $\mathrm{BCA}^{\mathrm{TM}}$ Protein Assay Kit (Thermo Scientific). The samples was denatured and separated by NuPAGE ${ }^{\circledR}$ Novex $^{\circledR}$ Bis-Tris Mini Gels (Invitrogen) at $200 \mathrm{~V}$ for 45 minutes at $4^{\circ} \mathrm{C}$, then transferred onto a nitrocellulose membrane (Invitrogen) using XCell II ${ }^{\mathrm{TM}}$ blot module (Invitrogen) at $30 \mathrm{~V}$ for 1 hour at $4^{\circ} \mathrm{C}$. The membranes were firstly blocked in SuperBlock (TBS) Blocking Buffer (Thermo Scientific), then incubated with the primary monoclonal antibodies at recommended dilution overnight at $4^{\circ} \mathrm{C}$. The membranes were incubated in the secondary antibody of Goat Anti-Mouse $\lg G(\mathrm{H}+\mathrm{L})$ and Goat Anti-Rabbit IgG $(\mathrm{H}+\mathrm{L})$ (Thermo Scientific) for 40 minutes at room temperature and exposed 
using SuperSignal ${ }^{\circledR}$ West Pico Chemiluminescent Substrate (Thermo Scientific) and CL-XPosure $^{\mathrm{TM}}$ Film (Thermo Scientific). The following primary antibodies were used in immunoblotting: integrin $\alpha_{2}$, integrin $\alpha_{5}$, cyclin D1, transforming growth factor - $\beta$ receptor II (TGF- $\beta$ RII), and phospho-TGF $\beta$ receptor II $\left(\mathrm{Tyr}^{424}\right)$ were purchased from Santa Cruz; integrin $\beta_{5}$, phospho-cyclin D1 $\left(\mathrm{Thr}^{286}\right)$, phospho-p44/42 MAPK (Erk1/2) $\left(\mathrm{Thr}^{202} / \mathrm{Tyr}^{204}\right), \mathrm{p} 44 / 42$ MAPK (Erk1/2), phospho-Src family $\left(\mathrm{Tyr}^{416}\right)$, and Src were from Cell Signaling; Sox9 and $\beta$-actin were from Abcam; type II collagen (CIIC1) was from DSHB.

\section{Statistical Analysis}

The Kruskal-Wallis test was used to determine significant differences among all groups, and Mann-Whitney U test was used for pairwise comparison. Statistical analyses were performed with SPSS 13.0 statistical software (SPSS Inc., Chicago, IL). P values less than 0.05 were considered statistically significant. 


\section{RESULTS}

ECM promoted hBMSC proliferative rate and induced directional migration

Passage 6 hBMSCs were expanded on conventional plastic flasks (Plastic) and flasks coated with extracellular matrix (ECM) at the initial density of 3,000 cells $/ \mathrm{cm}^{2}$ for 7 days. In regard to cell morphology, hBMSCs on Plastic appeared large and flattened, consistent with our previous study on SDSCs. In contrast, hBMSCs on ECM were smaller and spindle-like during the whole expansion period; the cells overlapped but maintained the same morphological appearance. It was observed that hBMSCs grown on ECM underwent directional migration along the ECM fibrils whereas the cells grown on Plastic showed random movement (Figure $6.1 A)$.

After 7-day expansion, hBMSCs on ECM yielded $16.8 \pm 2.3$ fold change of the cell number, while Plastic group produced $6.7 \pm 0.4$ fold increase (Figure $6.1 \mathrm{~B}$ ). The proliferation index of ECM group created by Proliferation Wizard Basic Model was $11.2 \pm 0.2$ and that of Plastic group was $5.3 \pm 0.1$ (Figure $6.1 \mathrm{C}$ ). The proliferation index is the sum of all hBMSC generations divided by the parent cells measured at the start of expansion.

ECM attenuated the stress of intracellular reactive oxygen species but increased apoptosis We examined the intracellular level of ROS stress in hBMSCs cultured on Plastic and ECM by staining the cells with DCF-DA. The hBMSCs cultured on Plastic showed a markedly higher level of DCF fluorescence compared with those cultured on ECM. At the start of passage 6 expansion, the mean fluorescence intensity (MFI) of DCF was $184 \pm 15$. At day 4 the MFI of Plastic group was $202 \pm 5$ which was 2.1-fold that on ECM (98 \pm 12$)$. At day 7 the MFI of Plastic group reached 241 \pm 15 which was 1.7-fold that on ECM (139 \pm 5$)$ (Figure 6.1D). In the regard to apoptosis analysis, however, ECM group showed higher apoptotic stress than Plastic group. The percentage of both annexin $\mathrm{V}$ and propidium iodide positive cells on ECM was $14 \%-144 \%$ higher than that on Plastic (Figure 6.1E).

Immunophenotype profile of hBMSCs on Plastic and ECM 
Flow cytometry was used to identify the immunophenotype profile of hBMSCs expanded on Plastic and ECM. Five-surface antigen expression level (Mean florescence intensity) increased in ECM group compared to Plastic group Figure 6.2A): SSEA-4 (100 vs. 48), CD71 (12 vs. 9), CD49b (integrin $\alpha_{2}, 40$ vs. 15), CD49d (integrin $\alpha_{4}, 15$ vs. 12) and integrin $\beta_{5}$ (374 vs.74). Ten-antigen expression level decreased after culturing on ECM (Figure 6.2B): CD90 (367 vs. 502), CD105 (177 vs. 273), CD166 (58 vs. 66), CD49a (integrin $\alpha_{1}, 12$ vs. 22), CD49c (integrin $\alpha_{3}$, 227 VS. 695), CD49e (integrin $\alpha_{5}, 68$ vs. 103), CD29 (integrin $\beta_{1}, 141$ vs. 210), and CD61 (integrin $\beta_{3}, 14$ vs. 19). However, seven-antigen expression level showed no difference between the two groups (Figure 6.2C): CD14, CD44, CD45, CD73, CD24, CD49f (integrin $\alpha_{6}$ ) and CD104 (integrin $\left.\beta_{4}\right)$.

Extracellular matrix induced changes of integrin and TGF $\beta$ receptor II expression and promoted phosphorylation of Src, ERK1/2 and cyclin D1

Immunoblotting analysis demonstrated that the expression of integrin $\alpha_{2}$ and $\beta_{5}$ was up-regulated when hBMSCs were expanded on ECM, while the level of integrin $\alpha_{5}$ was down-regulated (Figure 6.3), consistent with the immunophenotype profile. Interestingly, the expression of TGF- $\beta$ receptor II (TGF- $\beta$ RII) dramatically increased after treatment with ECM rather than Plastic, and TGF- $\beta$ receptor I showed the same tendency (data not shown). Considering the relationship between integrin and extracellular-signal regulated kinases (ERK), we tested the activity of Src kinase and ERK1/2. The phosphorylation level of Src and ERK1/2 was dramatically enhanced after hBMSC culturing on ECM compared with the control group (Plastic). The Src level was lower in ECM group at Day 5 but higher at Day 9, whereas ERK1/2 total protein content was continuously higher in ECM group. In order to illuminate the potential mechanism that ECM improved hBMSC proliferative rate, we used antibodies to probe the steady-level and phosphorylation of cyclin D1. After ECM expansion, we found a higher level of cyclin D1 expression and phosphorylation in BMSCs during the whole expansion period. Since the expression and activation of cyclin D1 was directly related to cell division and proliferation, the enhanced level of cyclin D1 induced by ECM was possibly responsible for the improved proliferative rate. 
ECM enhanced hBMSC chondrogenic differentiation potential

To compare the chondrogenic potentials of hBMSCs cultured on Plastic and ECM, cells were induced to differentiate in the pellet culture system by $10 \mathrm{ng} / \mathrm{mL}$ of TGF- $\beta 3$. After 15 -day differentiation, pellets of ECM group exhibited a larger size than those of Plastic group (Figure 6.4A). At Day 0 no positive histology staining was detectable in either groups (data not shown). At Day 7 Plastic group was barely positive for Alcian blue, which stained sulfated glycosaminoglycans (GAGs). However, pellets of ECM group were strongly positive for sulfated GAGs. The results of type II collagen immunohistochemistry were similar to those of Alcian blue staining, in which pellets cultured with ECM treatment displayed stronger expression than those on Plastic. Immunohistochemistry of type I collagen was nearly negative except for positive area on the edge of the pellets of ECM group. At Day 15 pellets of ECM group were more intensive in Alcian blue staining and type II and type I collagen immunohistochemistry than those of Plastic. Type $X$ collagen immunohistochemistry was negative in all pellets (Figure 6.4B).

Pellets of ECM group yielded more sulfated GAGs than Plastic group $(7.7 \mu \mathrm{g}$ vs. $4.7 \mu \mathrm{g}$ at Day 7 and $34.4 \mu \mathrm{g}$ vs. $9.9 \mu \mathrm{g}$ at Day 15) (Figure 6.5A). With respect to DNA amount, at Day $15 \mathrm{hBMSC}$ of ECM group maintained the DNA level better than Plastic group (79\% vs. $61 \%$ at Day 15) (Figure 6.5B). The chondrogenesis index, defined by the ratio of GAGs and DNA, illustrated the grade of hBMSC chondrogenic potential. Similar to the GAG amount, hBMSCs of ECM group showed stronger chondrogenic differentiation than the cells of Plastic group (3.6 vs. 1.6 at Day 7 and 16.6 vs. 3.8 at Day 15) (Figure 6.5C).

To obtain mechanical properties, chondrogenic pellets were examined for stiffness and Young's modulus. At Day 15, pellets of ECM group were $39.9 \pm 9.5 \mathrm{~N} / \mathrm{M}$ in stiffness compared with $24.9 \pm 4.7$ of Plastic (Figure 6.5D). In regard to Young's modulus, ECM group was $0.6 \pm$ $0.1 \mathrm{MPa}$ and Plastic group was $0.3 \pm 0.1 \mathrm{MPa}$ (Figure 6.5E).

Four typical chondrogenic marker genes were detected in hBMSCs expanded on ECM and Plastic. At the level of type II collagen mRNA expression, pellets of ECM group produced 
217-fold at Day 7 and 9-fold at Day 15 compared to Plastic group (Figure 6.6A). Aggrecan mRNA expression showed the same tendency; ECM group yielded a 5.5-fold increase compared with Plastic group at Day 7 (Figure 6.6B). Type I collagen was the chondrogenic maker at the early stage, which was expressed higher in ECM group at Day 7 but lower at Day 15 (Figure 6.6C). In regard to Sox9 mRNA level, pellets of Plastic group produced 5\% at Day 7 and 36\% at Day 14 more than ECM group (Figure 6.6D).

The level of phosphorylation of TGF- $\beta$ receptor II and expression of Sox 9 was enhanced after expansion on ECM in the process of chondrogenic differentiation In the expansion period, we found that hBMSCs expressed a higher level of TGF- $\beta$ RII after culturing on ECM. To determine if the high level of TGF- $\beta$ RIl would improve hBMSC chondrogenic potential, we tested the phosphorylated level of TGF- $\beta$ RII during chondrogenic induction. At Day 7 and Day 15, hBMSC pellets of ECM group showed a higher level of activation of TGF- $\beta$ RII than that of Plastic group. Sox9, as a chondrogenic-related transcription factor, had stronger expression in ECM group. The higher level of phosphorylation of TGF- $\beta$ RII and Sox9 would be responsible for the improvement of chondrogenic differentiation potential. Type II collagen was examined by immunoblotting and hBMSCs of ECM group yielded more amount than Plastic group, which was consistent with immunohistochemistry data. The activity of ERK1/2 was found suppressed in ECM group rather than Plastic group (Figure 6.7). 


\section{DISCUSSION}

In this study we expanded human bone marrow stem cells in conventional plastic flasks and on native 3D hBMSC-deposited extracellular matrix. Human BMSCs cultured on ECM had a higher proliferative rate, attenuated stress of intracellular ROS, a different immunophenotype profile from those expanded on Plastic, and gained an enhanced chondrogenic differentiation potential. We also explored the activity of several key signaling molecules of hBMSCs on Plastic and ECM during in vitro expansion and chondrogenesis. The results demonstrated that native 3D hBMSC-deposited ECM induced an increase of integrin $\alpha_{2}$ and $\beta_{5}$, and enhanced the activity of tyrosine kinase Src, the downstream of ERK1/2 and cyclin D1. At the same time, 3D ECM also elevated the expression of TGF- $\beta$ receptor II in hBMSCs and promoted the phosphorylation of TGF- $\beta$ receptor in chondrogenic differentiation.

Consistent with our previous study of synovium-derived stem cells (SDSCs), ${ }^{7}$ the 3D ECM improved hBMSC proliferative rate that was 2.5 -fold that of Plastic group. Cyclin D1 is proven to control the cell cycle progression through G1 phase and entry into S phase. ${ }^{22 ; 29}$ The steady-state level of activation of ERK1/2 is necessary for cyclin D1 phosphorylation and translocation, while the inhibition of ERK1/2 and the upstream kinases results in the decreased activity of cyclin D1. ${ }^{22 ; 23}$ Not only the expression level of cyclin D1 but also the phosphorylation was greatly increased in hBMSCs after expanding on 3D ECM; this could be one of the reasons for the higher proliferative rate induced by ECM. In this study the higher activation of cyclin D1 possibly resulted from increased Src kinase and ERK1/2. ${ }^{29}$ A similar 3D ECM deposited from NIH 3T3 cells also induced sustained activation of Ras and ERK1/2 through Src kinase and the blockage of Src resulted in suppression of ERK1/2. ${ }^{11}$

Intracellular ROS plays an important role in cell adhesion, migration, and proliferation and thus is essential for cell survival. ${ }^{30}$ The low concentration of ROS has a promotional effect on cell adhesion, whereas the high level of ROS would result in cell death. ${ }^{31}$ A high level of ROS has an inhibitory influence on cell proliferation by arresting the cell cycle at the $\mathrm{G} 1, \mathrm{~S}$, and $\mathrm{G} 2$

phases through down-regulation of cyclin D1 and D3 signaling. ${ }^{32}$ Kim et al. showed that an 
increase of intracellular ROS generation arrested the cell cycle at $G_{0}-G_{1}$ phase by inhibiting the cyclin D1/CDK4 complex. ${ }^{33}$ The attenuated level of ROS by 3D ECM was reported previously, ${ }^{34}$ and the suppression of ROS production resulted in the activation of FAK and SrC kinases to improve the cell adhesion and survival. ${ }^{35}$

The 3D ECM induced changes in the cell immunophenotype profile that was reported in our previous and other studies. ${ }^{7 ; 34}$ The cell surface marker expression is closely related to cell proliferation, differentiation and immunosuppression of mesenchymal stem cells. ${ }^{36}$ SSEA-4 that was originally discovered as an early embryonic glycolipid antigen in embryonic stem cells, was recently reported to express in mesenchymal stem cells and indicated that SSEA-4 would be a promising cell surface marker for cell identification and isolation ${ }^{37-39}$. In this study the increase of SSEA-4 positive cell population induced by 3D ECM might be correlated to the improved chondrogenic differentiation capacity, consistent with other studies. ${ }^{40}$ However, CD90 immunofluorescence intensity was attenuated in hBMSCs expanded on ECM which was inconsistent with our previous study on SDSCs; the difference could be due to the different MSC tissue sources. ${ }^{41-44}$

The interaction between ECM and cells is mainly dependent on integrin-induced signaling transduction. The conventional 2D ECM was proven to induce integrin activation through focal adhesion kinase (FAK), Src family kinase, and integrin-linked kinase (ILK) signaling pathways. ${ }^{16 ; 45-47}$ The FAK pathway was activated by integrin $\alpha_{5}, \alpha_{V}$, and $\beta_{1}$ in the process of cell adhesion to fibronectin and collagen substrates ${ }^{48}$ and the FAK autophosphorylation at Tyr ${ }^{397}$ to induce the activation of Src family kinase and mitogen-activated protein kinase (MAPK) cascades. $^{20 ; 49-52}$ However Damianova et al. reported on 3D ECM the autophosphorylated level of FAK was attenuated. ${ }^{11 ; 15}$ The three-dimensional structure and the complex components of the native cell-deposited ECM resulted in a distinct intracellular signaling pathway compared to that in an $2 \mathrm{D}$ conventional environment. ${ }^{11 ; 15 ; 16 ; 53}$ The increase of integrin $\alpha_{2}$ and $\beta_{5}$ and the decrease of integrin $\alpha_{5}$ and $\beta_{1}$ was confirmed by FACS and immunoblotting in this study. Integrin $\alpha_{2}$ and $\beta_{5}$ contribute significantly to cell focal adhesion and survival on fibrillar collagen substrate through Src and ERK-mediated signaling 
pathways. ${ }^{26 ; 54-56}$ Meanwhile the activated Src increased the expression of $\alpha_{2}$ integrin to improve cell survival. ${ }^{57}$ However, Src is also activated by other receptor kinases, ${ }^{27 ; 58 ; 59}$ so it is still unclear how the sustained activation of Src and ERK1/2 was induced by 3D ECM through certain specific integrins or other signaling receptors.

The greatly enhanced chondrogenic differentiation capacity of hBMSCs after expansion on 3D ECM was similar to our previous study on $\operatorname{SDSCs}^{7}$, adipose-derived stem cells and articular chondrocytes. We first demonstrated that the expression of TGF- $\beta$ receptor II was increased by the 3D ECM expansion system in hBMSCs. The high expression of TGF- $\beta$ receptor II in hBMSCs after culturing on ECM and the sustained steady-state phosphorylated level of TGF- $\beta$ receptor II induced by TGF- $\beta 1$ in chondrogenesis would be responsible for the strong expression of Sox 9 and type II collagen. ${ }^{60}$ Moreover, decorin, fibronectin, laminins, and type I and III collagens, identified in the 3D hBMSC-deposited $\mathrm{ECM}^{10 ; 34}$, played an important role in TGF- $\beta$ receptor responsiveness and modulating TGF- $\beta$ bioactivity during cell proliferation and differentiation ${ }^{61-64}$. Decorin was also reported to promote $\alpha_{2} \beta_{1}$ integrin-dependant cell adhesion and migration ${ }^{65}$ that was related in this study to the elevated level of integrin $\alpha_{2}$. The improved chondrogenic differentiation potential of BMSCs expanded on the 3D ECM is possibly due to the high responsiveness and sensitivity to TGF- $\beta$. Integrins also played a crucial role in chondrogenesis such as integrin $\beta_{1}{ }^{66}$ and interacted with type II collagen through integrin $\alpha_{2}$ when differentiating to mature chondrocytes ${ }^{67}$. The mechanism of elevated TGF- $\beta$ receptor II expression is still unclear but possibly related to the crosstalk between TGF- $\beta$ receptors and integrin. Reynolds et al. reported that, in dermal fibroblasts, the deficiency of integrin $\beta_{3}$ caused the increased expression of both TGF- $\beta$ receptor I and receptor $11 .{ }^{68}$ Myofibroblastic differentiation in dermal fibroblasts was induced by $\alpha_{\mathrm{v}} \beta_{5}$ integrin because of the recruitment of latent TGF- $\beta 1$ and activation of TGF- $\beta$ receptors. ${ }^{69}$ However the mechanism of integrin interaction with TGF- $\beta$ receptors induced by 3D ECM remains to be established.

The role of ERK1/2 in chondrogenic differentiation is controversial. The conventional opinion is that MAPK cascade was activated by TGF- $\beta$ and the phosphorylated ERK1/2 promoted the 
expression of chondrogenic genes such as aggrecan and type II collagen. ${ }^{70 ; 71}$ However recent research suggested that the inhibition of $E R K 1 / 2^{72-74}$ or $\mathrm{JNK}^{75}$ can improve the chondrogenic differentiation of MSCs that were considered as two important TGF- $\beta$ non-Smad signaling pathways. We found similar decreased activity of ERK1/2 during the chondrogenic differentiation especially in hBMSCs expanded on ECM. The possible explanation is that the activation of ERK1/2 initiates the transcription of chondrogenic-specific genes such as aggrecan and type II collagen but, in the long-term induction to chondrogenesis, ERK1/2 would give way to other signaling pathways such as PI3K/Akt to generate cartilage-specific matrix. ${ }^{73 ; 75}$

In summary our study demonstrated that the utilization of the new 3D cell-deposited ECM on hBMSC in vitro expansion was able to dramatically increase the cell proliferative rate, attenuate the stress of intracellular ROS, and improve the chondrogenic differentiation potential. We demonstrated that 3D ECM induced sustained activation of Src kinase and ERK1/2 and phosphorylated cyclin D1 to promote the cell-cycle and proliferation. The enhanced chondrogenic differentiation ability of hBMSCs by the 3D ECM was because of the high expression and sensitivity of TGF- $\beta$ receptor II. Due to the complexity of the 3D ECM, the cell signaling transduction is different from that in 2D monolayer culture condition and needs to be illuminated in the future. 


\section{ACKOWLWDGEMENTS}

The authors thank Suzanne Smith for her help in editing the manuscript and Dr. Kathleen Brundage for her help with the experiments using flow cytometry, which were performed in the West Virginia University Flow Cytometry Core Facility and supported in part by NIH grants RR106440 


\section{REFERENCES}

1. Friedenstein AJ, Gorskaja JF, Kulagina NN. Fibroblast precursors in normal and irradiated mouse hematopoietic organs. Exp.Hematol. 1976;4:267-74.

2. Liechty KW, MacKenzie TC, Shaaban AF et al. Human mesenchymal stem cells engraft and demonstrate site-specific differentiation after in utero transplantation in sheep. Nat.Med. 2000;6:1282-6.

3. Pei M, He F, Vunjak-Novakovic G. Synovium-derived stem cell-based chondrogenesis. Differentiation 2008;76:1044-56.

4. Pereira RF, Halford KW, O'Hara MD et al. Cultured adherent cells from marrow can serve as long-lasting precursor cells for bone, cartilage, and lung in irradiated mice. Proc.Natl.Acad.Sci.U.S.A 1995;92:4857-61.

5. Sakaguchi Y, Sekiya I, Yagishita K et al. Comparison of human stem cells derived from various mesenchymal tissues: superiority of synovium as a cell source. Arthritis Rheum. 2005;52:2521-9.

6. Jones EA, Kinsey SE, English A et al. Isolation and characterization of bone marrow multipotential mesenchymal progenitor cells. Arthritis Rheum. 2002;46:3349-60.

7. He F, Chen X, Pei M. Reconstruction of an in vitro tissue-specific microenvironment to rejuvenate synovium-derived stem cells for cartilage tissue engineering. Tissue Eng Part A 2009;15:3809-21.

8. Castello-Cros R, Cukierman E. Stromagenesis during tumorigenesis: characterization of tumor-associated fibroblasts and stroma-derived 3D matrices. Methods Mol.Biol. 2009;522:275-305.

9. Castello-Cros R, Khan DR, Simons J et al. Staged stromal extracellular 3D matrices differentially regulate breast cancer cell responses through PI3K and beta1-integrins. 
BMC.Cancer 2009;9:94.

10. Chen XD, Dusevich $\vee$, Feng JQ et al. Extracellular matrix made by bone marrow cells facilitates expansion of marrow-derived mesenchymal progenitor cells and prevents their differentiation into osteoblasts. J.Bone Miner.Res. 2007;22:1943-56.

11. Damianova R, Stefanova N, Cukierman E et al. Three-dimensional matrix induces sustained activation of ERK1/2 via Src/Ras/Raf signaling pathway. Cell Biol.Int. 2008;32:229-34.

12. Dao MA, Hashino $\mathrm{K}$, Kato I et al. Adhesion to fibronectin maintains regenerative capacity during ex vivo culture and transduction of human hematopoietic stem and progenitor cells. Blood 1998;92:4612-21.

13. Eslaminejad MB, Mirzadeh H, Nickmahzar A et al. Type I collagen gel in seeding medium improves murine mesencymal stem cell loading onto the scaffold, increases their subsequent proliferation, and enhances culture mineralization. J.Biomed.Mater.Res.B Appl.Biomater. 2009;90:659-67.

14. Klees RF, Salasznyk RM, Kingsley $\mathrm{K}$ et al. Laminin-5 induces osteogenic gene expression in human mesenchymal stem cells through an ERK-dependent pathway. Mol.Biol.Cell 2005;16:881-90.

15. Cukierman E, Pankov R, Stevens DR et al. Taking cell-matrix adhesions to the third dimension. Science 2001;294:1708-12.

16. Giancotti FG, Ruoslahti E. Integrin signaling. Science 1999;285:1028-32.

17. Heino J, Kapyla J. Cellular receptors of extracellular matrix molecules. Curr.Pharm.Des 2009;15:1309-17.

18. Yamada KM, Pankov R, Cukierman E. Dimensions and dynamics in integrin function. Braz.J.Med.Biol.Res. 2003;36:959-66. 
19. Zhu X, Assoian RK. Integrin-dependent activation of MAP kinase: a link to shape-dependent cell proliferation. Mol.Biol.Cell 1995;6:273-82.

20. Schlaepfer DD, Hanks SK, Hunter $\mathrm{T}$ et al. Integrin-mediated signal transduction linked to Ras pathway by GRB2 binding to focal adhesion kinase. Nature 1994;372:786-91.

21. Zhao JH, Reiske H, Guan JL. Regulation of the cell cycle by focal adhesion kinase. J.Cell Biol. 1998;143:1997-2008.

22. Roovers K, Davey G, Zhu X et al. Alpha5beta1 integrin controls cyclin D1 expression by sustaining mitogen-activated protein kinase activity in growth factor-treated cells. Mol.Biol.Cell 1999;10:3197-204.

23. Weber JD, Raben DM, Phillips $P J$ et al. Sustained activation of extracellular-signal-regulated kinase 1 (ERK1) is required for the continued expression of cyclin D1 in G1 phase. Biochem.J. 1997;326 (Pt 1):61-8.

24. Gordon MY. Extracellular matrix of the marrow microenvironment. Br.J.Haematol. $1988 ; 70: 1-4$

25. Bi Y, Ehirchiou D, Kilts TM et al. Identification of tendon stem/progenitor cells and the role of the extracellular matrix in their niche. Nat.Med. 2007;13:1219-27.

26. Holtkotter $\mathrm{O}$, Nieswandt $\mathrm{B}$, Smyth $\mathrm{N}$ et al. Integrin alpha 2-deficient mice develop normally, are fertile, but display partially defective platelet interaction with collagen. J.Biol.Chem. 2002;277:10789-94.

27. Cabodi S, Moro L, Bergatto $\mathrm{E}$ et al. Integrin regulation of epidermal growth factor (EGF) receptor and of EGF-dependent responses. Biochem.Soc.Trans. 2004;32:438-42.

28. Rodriguez F, Patel SK, Cohen C. Measuring the modulus of a sphere by squeezing between parallel plates. Journal of Applied Polymer Science 1990;5:285-95. 
29. Jirmanova L, Afanassieff M, Gobert-Gosse $S$ et al. Differential contributions of ERK and PI3-kinase to the regulation of cyclin D1 expression and to the control of the G1/S transition in mouse embryonic stem cells. Oncogene 2002;21:5515-28.

30. Rollet-Labelle E, Grange MJ, Elbim C et al. Hydroxyl radical as a potential intracellular mediator of polymorphonuclear neutrophil apoptosis. Free Radic.Biol.Med. 1998;24:563-72.

31. Lamari F, Braut-Boucher F, Pongnimitprasert $\mathrm{N}$ et al. Cell adhesion and integrin expression are modulated by oxidative stress in EA.hy 926 cells. Free Radic.Res. $2007 ; 41: 812-22$.

32. Barnouin K, Dubuisson ML, Child ES et al. H2O2 induces a transient multi-phase cell cycle arrest in mouse fibroblasts through modulating cyclin D and p21Cip1 expression. J.Biol.Chem. 2002;277:13761-70.

33. Kim $\mathrm{TH}$, Oh S, Kim SS. Recombinant human prothrombin kringle-2 induces bovine capillary endothelial cell cycle arrest at G0-G1 phase through inhibition of cyclin D1/CDK4 complex: modulation of reactive oxygen species generation and up-regulation of cyclin-dependent kinase inhibitors. Angiogenesis. 2005;8:307-14.

34. Lai Y, Sun Y, Skinner CM et al. Reconstitution of Marrow-derived Extracellular Matrix Ex Vivo: a Robust Culture System for Expanding Large-scale Highly Functional Human Mesenchymal Stem Cells. Stem Cells Dev. 2009.

35. Song $\mathrm{H}$, Cha $\mathrm{MJ}$, Song BW et al. Reactive oxygen species inhibit adhesion of mesenchymal stem cells implanted into ischemic myocardium via interference of focal adhesion complex. Stem Cells 2010;28:555-63.

36. Pittenger MF, Mackay AM, Beck SC et al. Multilineage potential of adult human mesenchymal stem cells. Science 1999;284:143-7.

37. Foster LJ, Zeemann PA, Li C et al. Differential expression profiling of membrane 
proteins by quantitative proteomics in a human mesenchymal stem cell line undergoing osteoblast differentiation. Stem Cells 2005;23:1367-77.

38. Kawanabe $\mathrm{N}$, Murata $\mathrm{S}$, Murakami $\mathrm{K}$ et al. Isolation of multipotent stem cells in human periodontal ligament using stage-specific embryonic antigen-4. Differentiation 2010;79:74-83.

39. Riekstina U, Cakstina I, Parfejevs $\vee$ et al. Embryonic stem cell marker expression pattern in human mesenchymal stem cells derived from bone marrow, adipose tissue, heart and dermis. Stem Cell Rev. 2009;5:378-86.

40. Gang EJ, Bosnakovski D, Figueiredo CA et al. SSEA-4 identifies mesenchymal stem cells from bone marrow. Blood 2007;109:1743-51.

41. Campioni D, Rizzo R, Stignani M et al. A decreased positivity for CD90 on human mesenchymal stromal cells (MSCs) is associated with a loss of immunosuppressive activity by MSCs. Cytometry B Clin.Cytom. 2009;76:225-30.

42. Djouad F, Bony $\mathrm{C}$, Haupl $\mathrm{T}$ et al. Transcriptional profiles discriminate bone marrow-derived and synovium-derived mesenchymal stem cells. Arthritis Res. Ther. 2005;7:R1304-R1315.

43. Nasef A, Mathieu N, Chapel A et al. Immunosuppressive effects of mesenchymal stem cells: involvement of HLA-G. Transplantation 2007;84:231-7.

44. Rizzo R, Campioni D, Stignani $M$ et al. A functional role for soluble HLA-G antigens in immune modulation mediated by mesenchymal stromal cells. Cytotherapy. 2008;10:364-75.

45. Guan JL, Shalloway D. Regulation of focal adhesion-associated protein tyrosine kinase by both cellular adhesion and oncogenic transformation. Nature 1992;358:690-2.

46. Wary KK, Mainiero F, Isakoff SJ et al. The adaptor protein Shc couples a class of 
integrins to the control of cell cycle progression. Cell 1996;87:733-43.

47. Xing Z, Chen HC, Nowlen JK et al. Direct interaction of V-Src with the focal adhesion kinase mediated by the Src SH2 domain. Mol.Biol.Cell 1994;5:413-21.

48. Green JA, Yamada KM. Three-dimensional microenvironments modulate fibroblast signaling responses. Adv.Drug Deliv.Rev. 2007;59:1293-8.

49. Chen HC, Appeddu PA, Isoda $\mathrm{H}$ et al. Phosphorylation of tyrosine 397 in focal adhesion kinase is required for binding phosphatidylinositol 3-kinase. J.Biol.Chem. 1996;271:26329-34.

50. Parsons JT, Schaller MD, Hildebrand J et al. Focal adhesion kinase: structure and signalling. J.Cell Sci.Suppl 1994;18:109-13.

51. Schaller MD, Hildebrand JD, Shannon JD et al. Autophosphorylation of the focal adhesion kinase, pp125FAK, directs SH2-dependent binding of pp60src. Mol.Cell Biol. 1994;14:1680-8.

52. Schlaepfer DD, Broome MA, Hunter T. Fibronectin-stimulated signaling from a focal adhesion kinase-c-Src complex: involvement of the Grb2, p130cas, and Nck adaptor proteins. Mol.Cell Biol. 1997;17:1702-13.

53. Cukierman E, Pankov R, Yamada KM. Cell interactions with three-dimensional matrices. Curr.Opin.Cell Biol. 2002;14:633-9.

54. Sawhney RS, Cookson MM, Omar $Y$ et al. Integrin alpha2-mediated ERK and calpain activation play a critical role in cell adhesion and motility via focal adhesion kinase signaling: identification of a novel signaling pathway. J.Biol.Chem. 2006;281:8497-510.

55. Inoue O, Suzuki-Inoue K, Dean WL et al. Integrin alpha2beta1 mediates outside-in regulation of platelet spreading on collagen through activation of Src kinases and PLCgamma2. J.Cell Biol. 2003;160:769-80. 
56. Eliceiri BP, Puente XS, Hood JD et al. Src-mediated coupling of focal adhesion kinase to integrin alpha(v)beta5 in vascular endothelial growth factor signaling. J.Cell Biol. 2002;157:149-60.

57. Park HB, Golubovskaya V, Xu L et al. Activated Src increases adhesion, survival and alpha2-integrin expression in human breast cancer cells. Biochem.J. 2004;378:559-67.

58. Moro L, Dolce L, Cabodi S et al. Integrin-induced epidermal growth factor (EGF) receptor activation requires $\mathrm{c}-\mathrm{Src}$ and $\mathrm{p} 130 \mathrm{Cas}$ and leads to phosphorylation of specific EGF receptor tyrosines. J.Biol.Chem. 2002;277:9405-14.

59. Lee JW, Juliano R. Mitogenic signal transduction by integrin- and growth factor receptor-mediated pathways. Mol.Cells 2004;17:188-202.

60. Grimaud E, Heymann D, Redini F. Recent advances in TGF-beta effects on chondrocyte metabolism. Potential therapeutic roles of TGF-beta in cartilage disorders. Cytokine Growth Factor Rev. 2002;13:241-57.

61. Li X, McFarland DC, Velleman SG. Extracellular matrix proteoglycan decorin-mediated myogenic satellite cell responsiveness to transforming growth factor-beta1 during cell proliferation and differentiation Decorin and transforming growth factor-beta1 in satellite cells. Domest.Anim Endocrinol. 2008;35:263-73.

62. Ferdous Z, Wei VM, Iozzo R et al. Decorin-transforming growth factor- interaction regulates matrix organization and mechanical characteristics of three-dimensional collagen matrices. J.Biol.Chem. 2007;282:35887-98.

63. Bi $\mathrm{Y}$, Stuelten $\mathrm{CH}$, Kilts $\mathrm{T}$ et al. Extracellular matrix proteoglycans control the fate of bone marrow stromal cells. J.Biol.Chem. 2005;280:30481-9.

64. Li X, McFarland DC, Velleman SG. Effect of transforming growth factor-beta on decorin and beta1 integrin expression during muscle development in chickens. 
Poult.Sci. 2006;85:326-32.

65. Fiedler LR, Schonherr E, Waddington $\mathrm{R}$ et al. Decorin regulates endothelial cell motility on collagen I through activation of insulin-like growth factor I receptor and modulation of alpha2beta1 integrin activity. J.Biol.Chem. 2008;283:17406-15.

66. Shakibaei M. Inhibition of chondrogenesis by integrin antibody in vitro. Exp.Cell Res. 1998;240:95-106.

67. Loeser RF. Chondrocyte integrin expression and function. Biorheology 2000;37:109-16.

68. Reynolds LE, Conti FJ, Lucas $M$ et al. Accelerated re-epithelialization in beta3-integrin-deficient- mice is associated with enhanced TGF-beta1 signaling. Nat.Med. 2005;11:167-74.

69. Asano $\mathrm{Y}$, Ihn $\mathrm{H}$, Yamane $\mathrm{K}$ et al. Increased expression of integrin alphavbeta5 induces the myofibroblastic differentiation of dermal fibroblasts. Am.J.Pathol. 2006;168:499-510.

70. Watanabe $\mathrm{H}$, de Caestecker MP, Yamada Y. Transcriptional cross-talk between Smad, ERK1/2, and p38 mitogen-activated protein kinase pathways regulates transforming growth factor-beta-induced aggrecan gene expression in chondrogenic ATDC5 cells. J.Biol.Chem. 2001;276:14466-73.

71. Zhang YE. Non-Smad pathways in TGF-beta signaling. Cell Res. 2009;19:128-39.

72. Bobick BE, Kulyk WM. The MEK-ERK signaling pathway is a negative regulator of cartilage-specific gene expression in embryonic limb mesenchyme. J.Biol.Chem. 2004;279:4588-95.

73. Kim HJ, Im GI. The effects of ERK1/2 inhibitor on the chondrogenesis of bone marrow- and adipose tissue-derived multipotent mesenchymal stromal cells. Tissue Eng Part A 2010;16:851-60. 
74. Li J, Zhao Z, Liu J et al. MEK/ERK and p38 MAPK regulate chondrogenesis of rat bone marrow mesenchymal stem cells through delicate interaction with TGF-beta1/Smads pathway. Cell Prolif. 2010;43:333-43.

75. Caerteling SB, van der Kraan PM, Vitters EL et al. Inhibition of JNK enhances chondrogenesis of mesenchymal progenitor cells by increasing autocrine TGFbeta signaling. Arthritis Rheum. 2010. 


\section{FIGURE LEDGENS}

Figure 6.1 Extracellular matrix (ECM) induces changes in the human bone marrow stem cells (hBMSCs) affacting cell morphology, proliferative rate, intracellular reactive oxygen species (ROS) and apoptosis level in the period of monolayer expansion. (A) Representative phase contrast images of hBMSC in the middle of expansion; (B) hBMSC cell number fold change after 7-day monolayer expansion under Plastic and ECM culture conditions; (C) the proliferation index of hBMSC on Plastic and ECM analyzed by flow cytometry; (D) intracellular ROS level decreases when culturing on ECM; (E) the percentage of apoptotic cells varies differently on Plastic and ECM. Data are shown as average \pm SD.

Figure 6.2 Immunophenotype profile of hBMSCs cultured on Plastic and ECM. (A) hBMSCs cultured on ECM showed increase in the positive-percentage and fluorescence intensity of SSEA-4, CD71, CD49b, CD49d, and integrin 35 ; (B) hBMSCs cultured on Plastic have higher expression of CD73, CD90, CD105, CD166, CD44, CD49a, CD49c, CD49e, CD29 and CD61; (C) the expression level of CD14, CD45, CD24, CD49f and CD104 shows no difference between Plastic and ECM groups.

Figure 6.3 Immunoblotting of hBMSCs when expanding on Plastic and ECM. Total lysates of hBMSCs were detected to the antibodies of integrin $\alpha_{2}$, integrin $\beta_{5}$, integrin $\alpha_{5}$, transforming growth factor $\beta$ receptor II (TGF $\beta$ receptor II). The lysates were also probed to phospho-Cyclin D1 $\left(\mathrm{Thr}^{286}\right)$, Cyclin D1, phospho-Src family $\left(\mathrm{Tyr}^{416}\right)$, Src, phospho-p44/42 MAPK (Erk1/2) $\left(\mathrm{Thr}^{202} / \mathrm{Tyr}^{204}\right)$, and p44/42 MAPK (Erk1/2). $\beta$ actin was used as an internal control for protein loading.

Figure 6.4 Phase image, histology and immunohistochemistry of hBMSC pellets under chondrogenic stimulation. (A) typical hBMSC pellets of Plastic and ECM groups phase images; (B) Alcian blue staining was used to detect sulfated glycosaminoglycans (GAGs), and immunohistochemistry (IHC) was for type I (COL I), type II (COL II) and type X (COL X) collagens. 
Figure 6.5 Biochemistry to analyze glycosaminoglycans (GAGs) and DNA content of hBMSCs chondrogenic differentiation of Plastic and ECM groups. (A), GAGs of hBMSC pellets were measured at day 0 , day 7 and day 15; (B), DNA content of hBMSC pellets changes during 15 days normalized by the content of pellets at day 0 ; $(C)$, chondrogenesis index (the ratio of GAGs and DNA) represented the degree of chondrogenic differentiation of hBMSCs; mechanical testing was used to examine the stiffness (D) and Young's modulus (E) of hBMSC pellet after chondrogenic differentiation. Data are shown as average \pm SD for $n=5$ for DNA and GAGs analysis and $n=4$ for mechanical testing.

Figure 6.6 (A) to (D) Real-time PCR to examine the chondrogenic marker gene expression level (type I and II collagen, aggrecan, sox-9) normalized by 18S rRNA. (E) and (F) the hypertrophic gene expression (type X collagen and MMP13) was tested in hBMSC pellets. Data are shown as average \pm SD for $n=4$.

Figure 6.7 Immunoblotting of hBMSC chondrogenic pellets was used to detect phospho-TGF $\beta$ receptor II (Tyr 424), Sox9, type II collagen, phosphorylated ERK1/2 and ERK1/2 expression level. $\beta$ actin was used as an internal control for protein loading. 
Figure 6.1
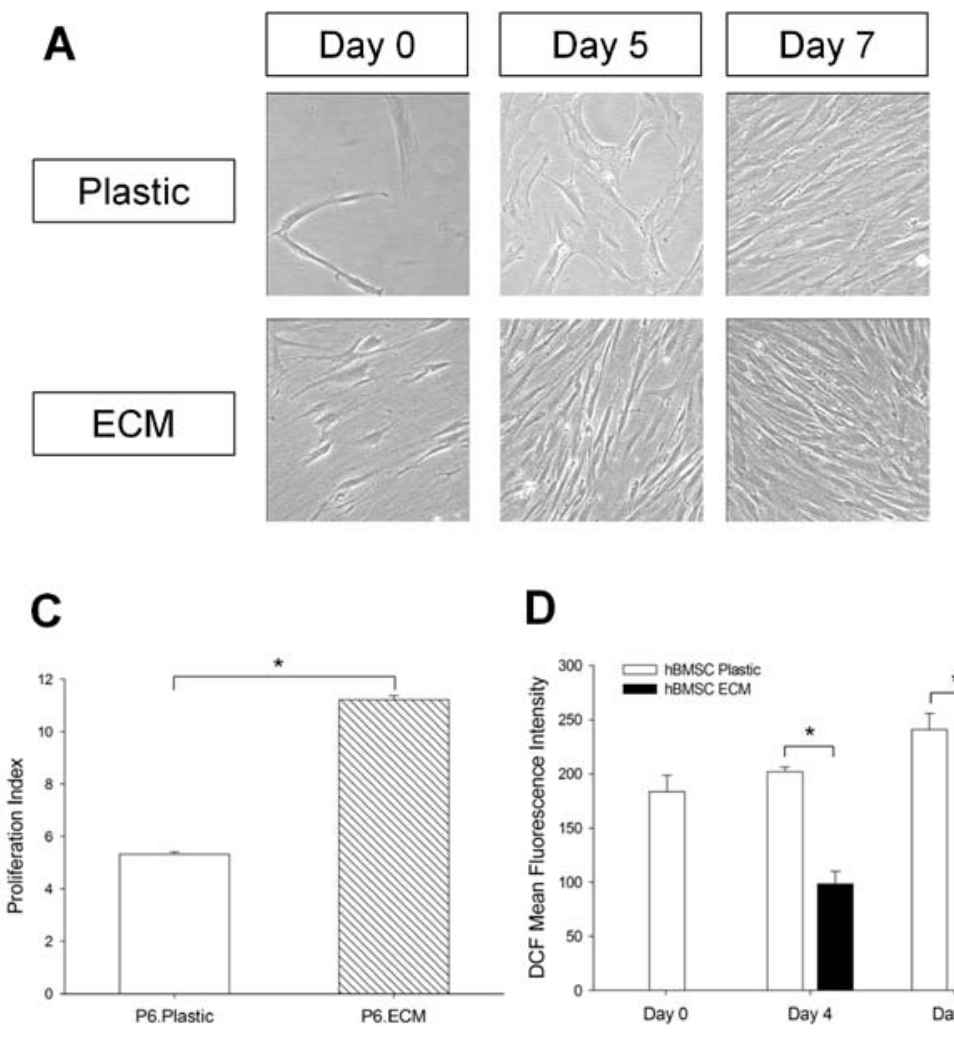

D

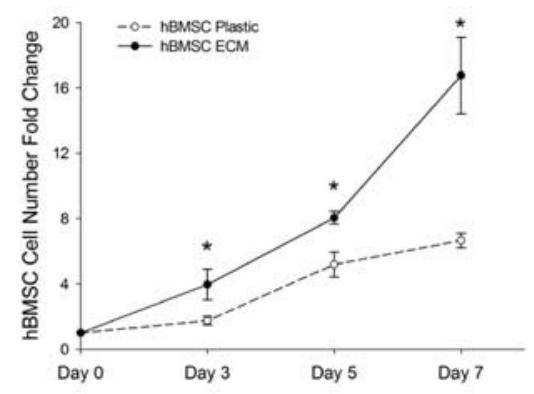

E

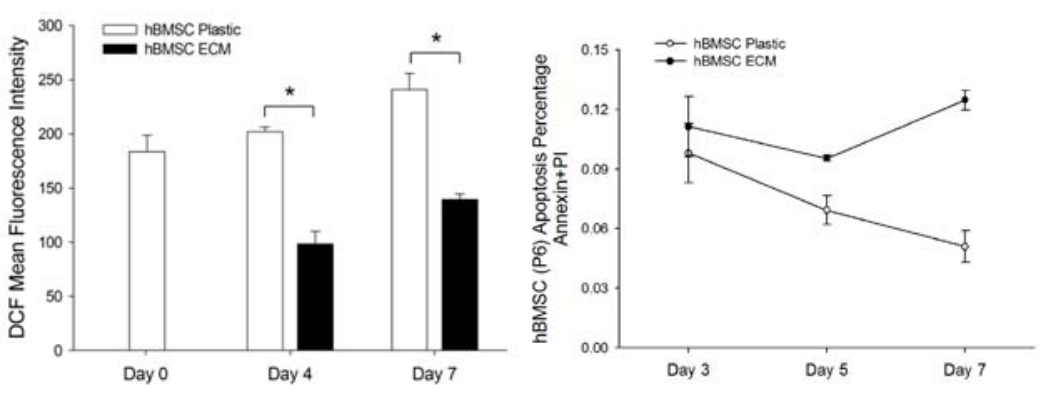


Figure 6.2

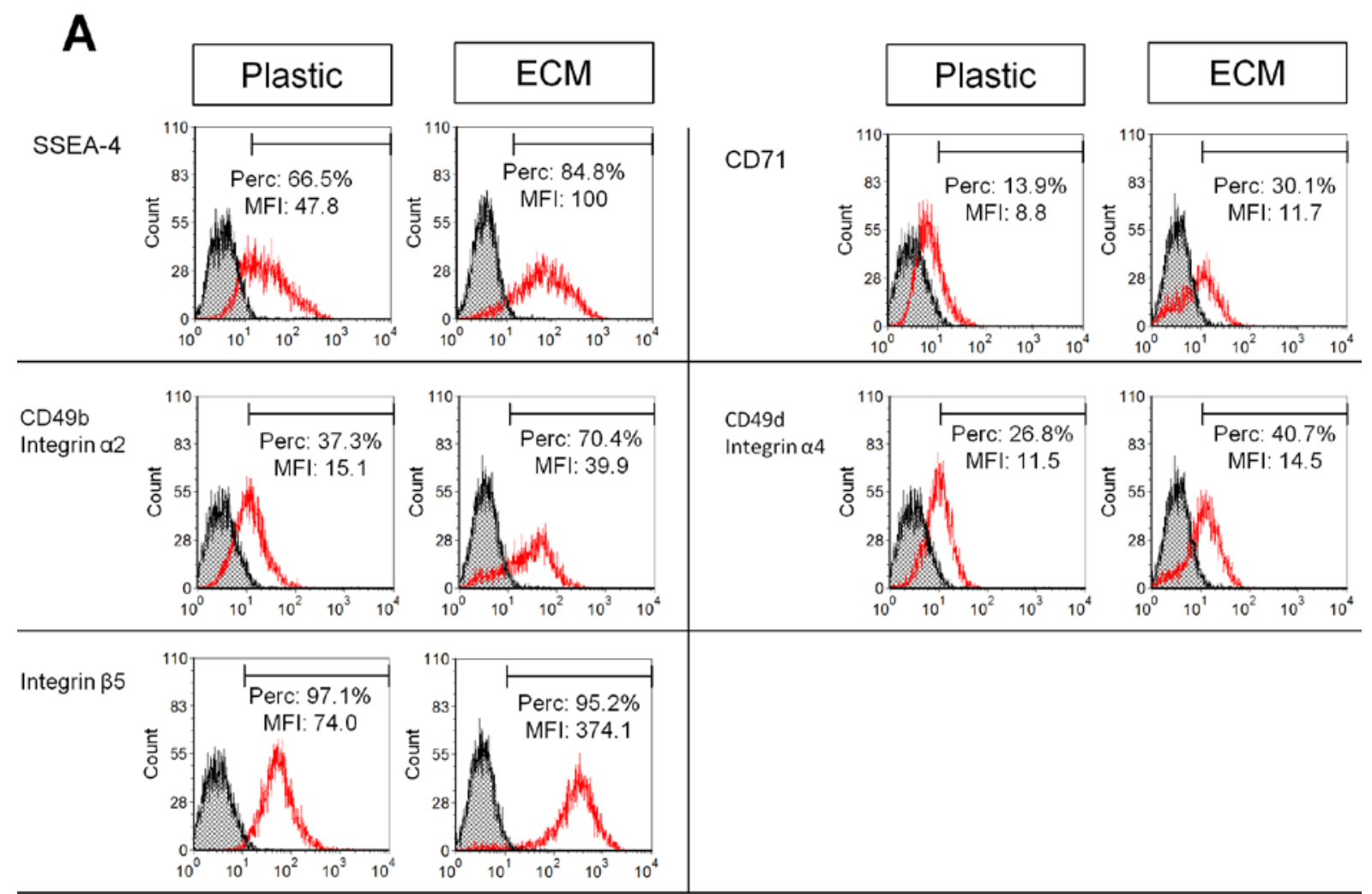




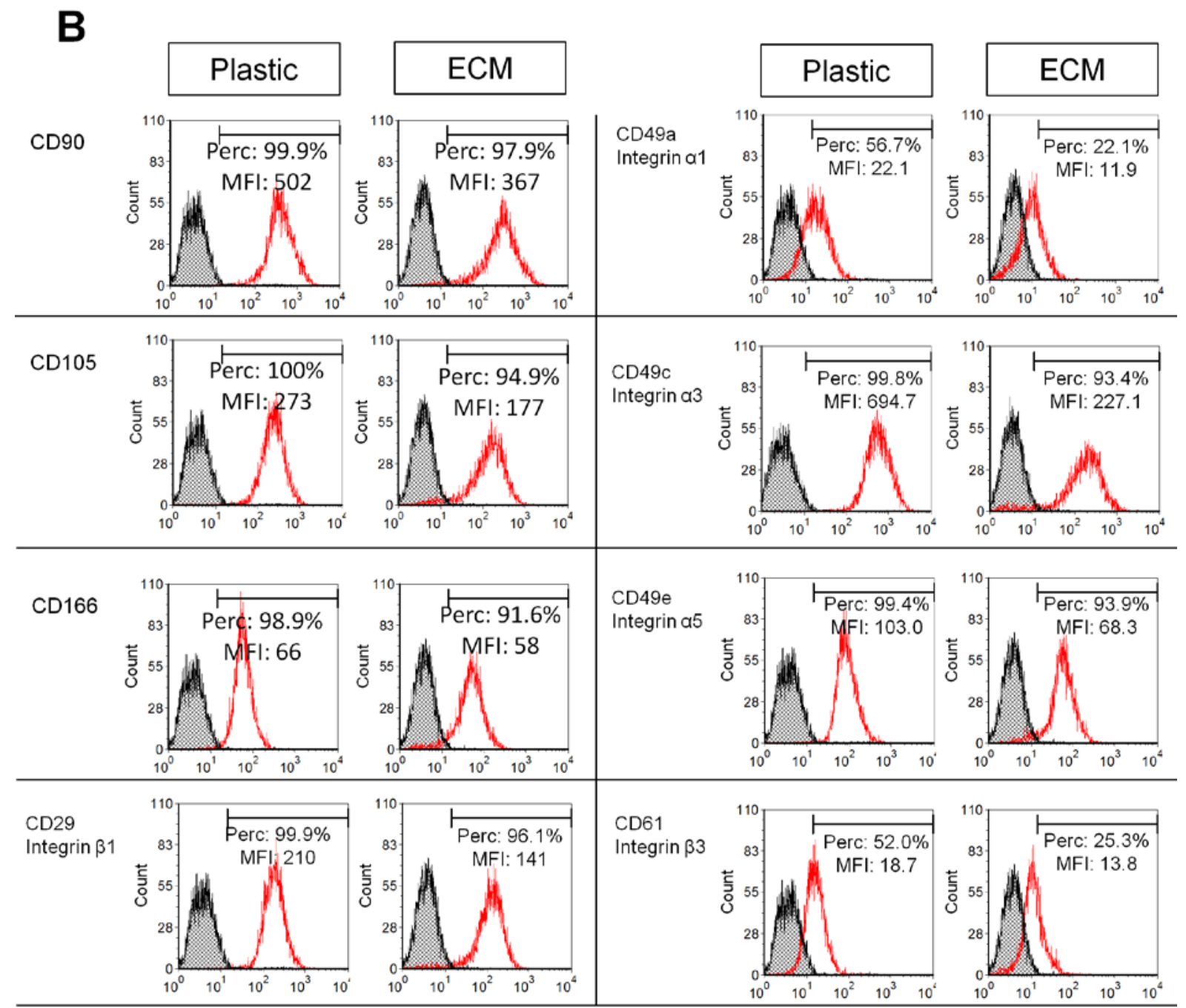




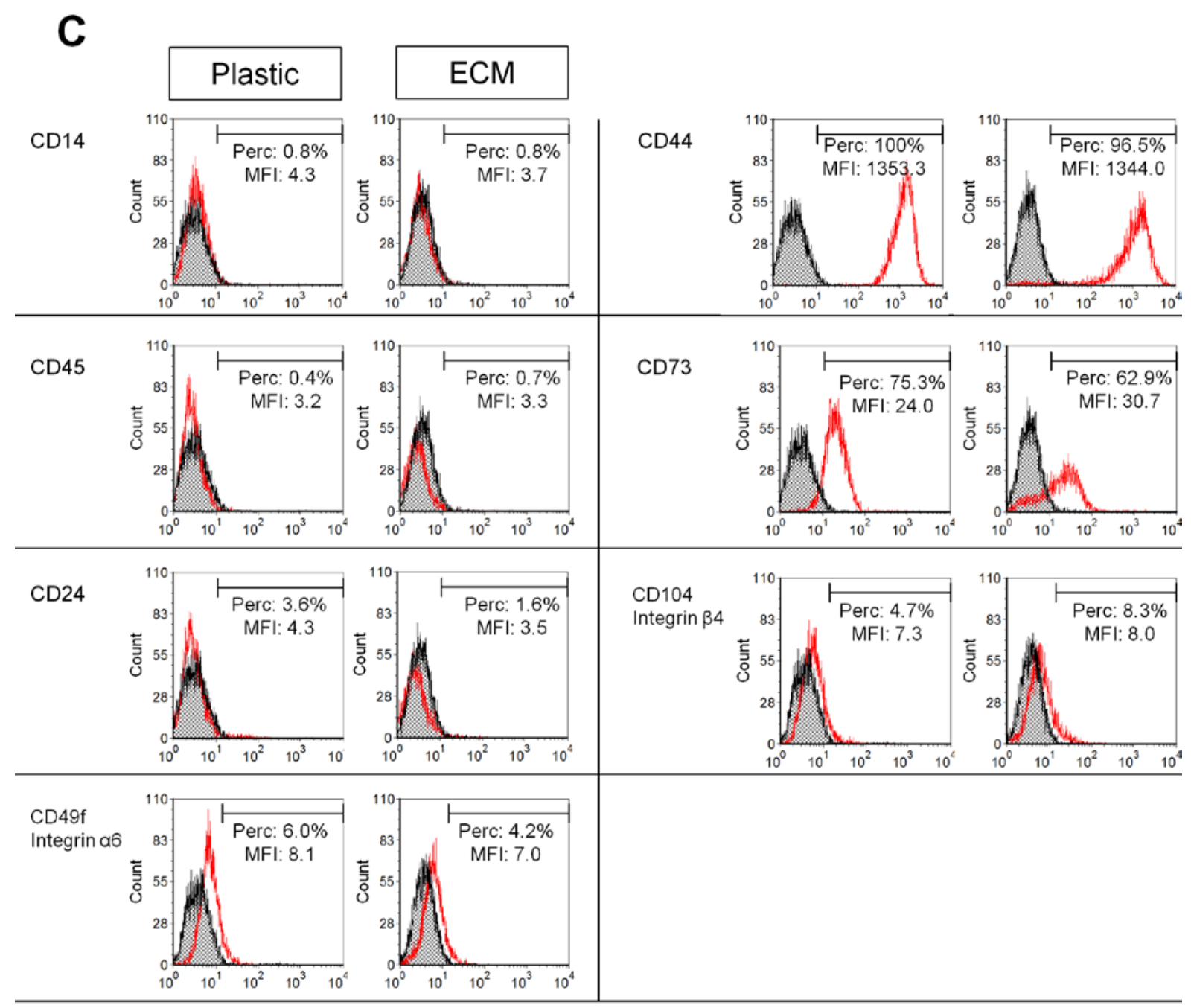


Figure 6.3

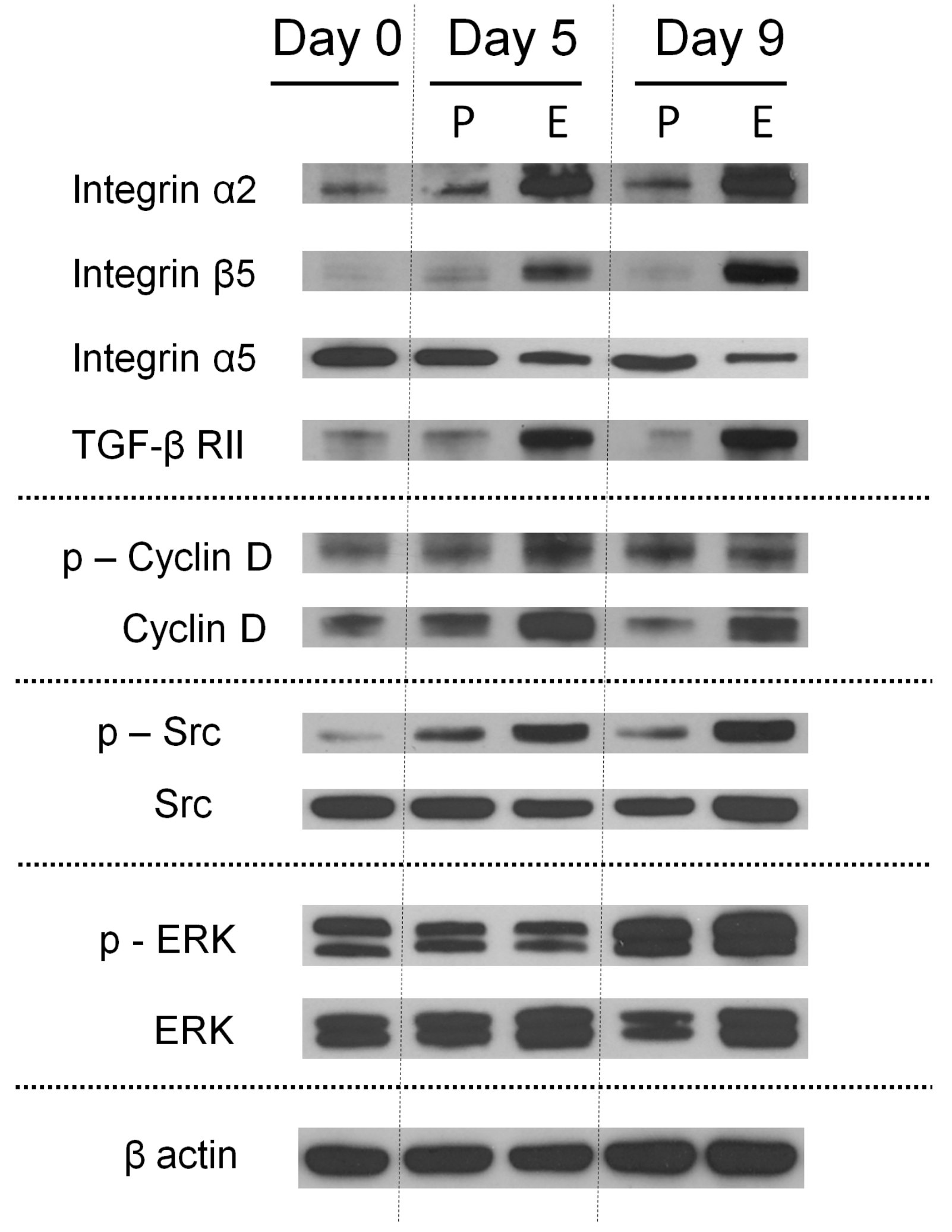


Figure 6.4

A
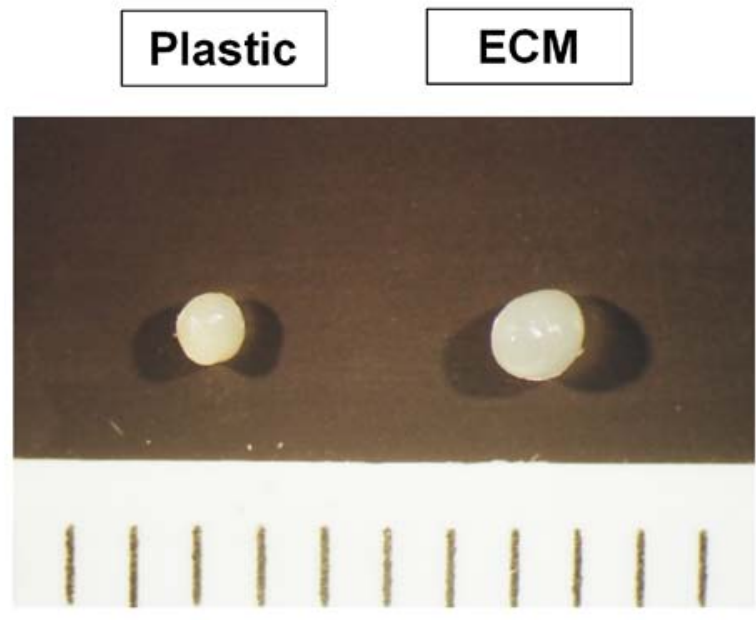

B

\section{Alcian Blue}
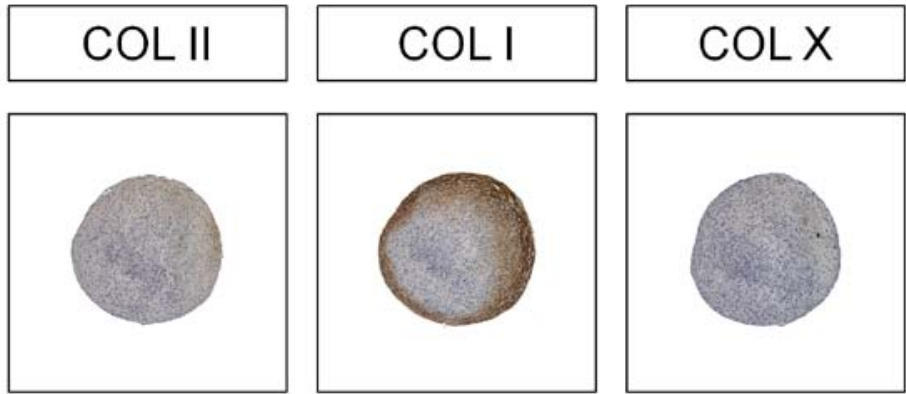

\section{Plastic}

ECM
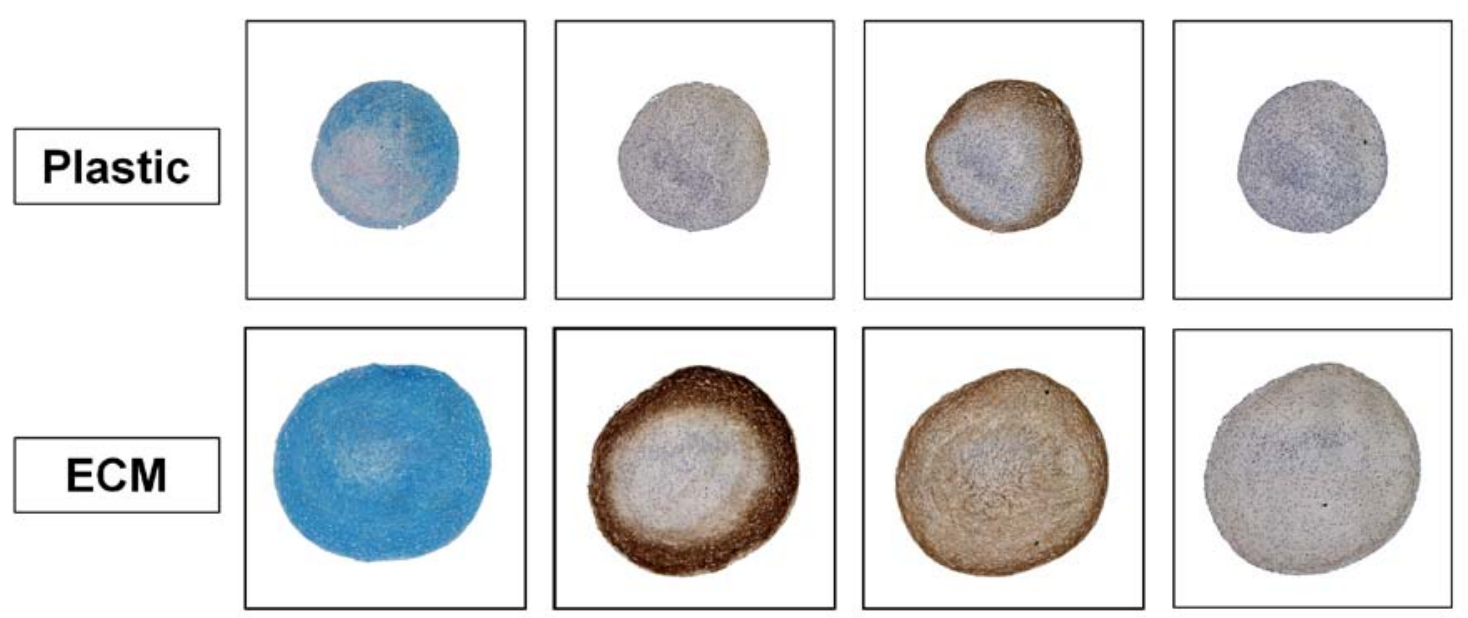
Figure 6.5

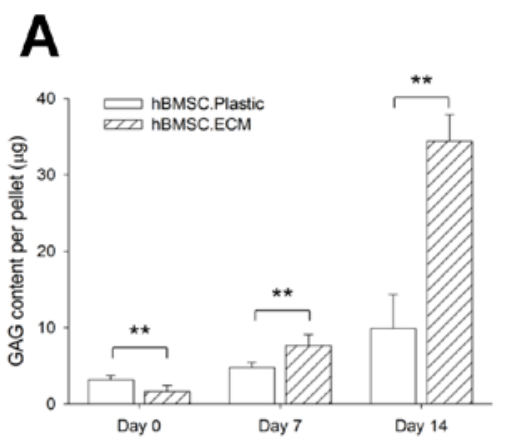

B

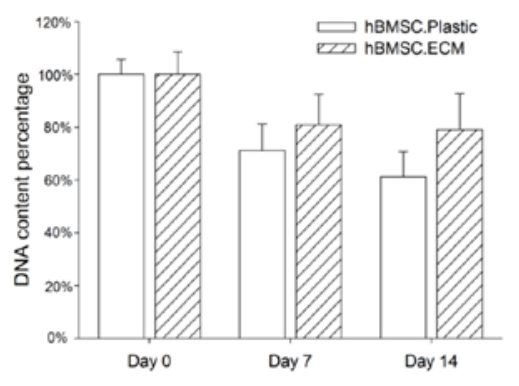

C

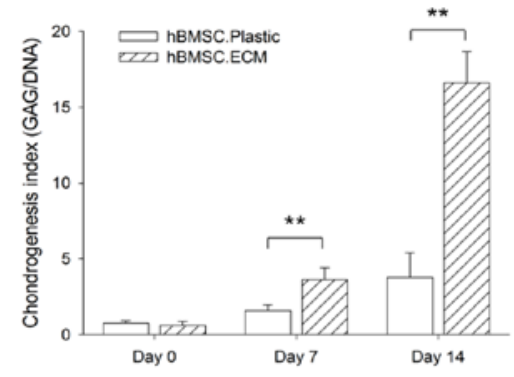

D

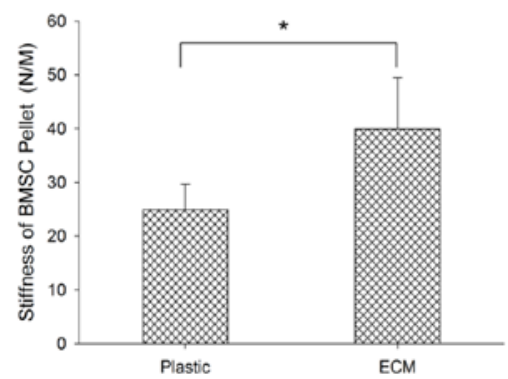

E

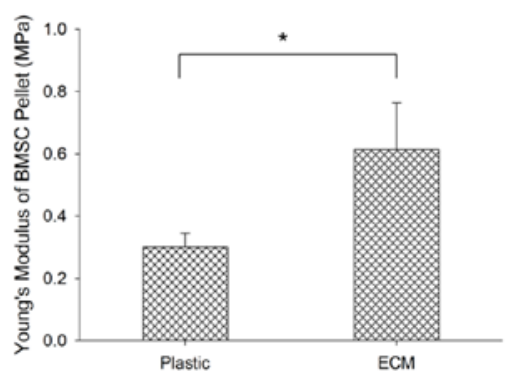


Figure 6.6

A

B
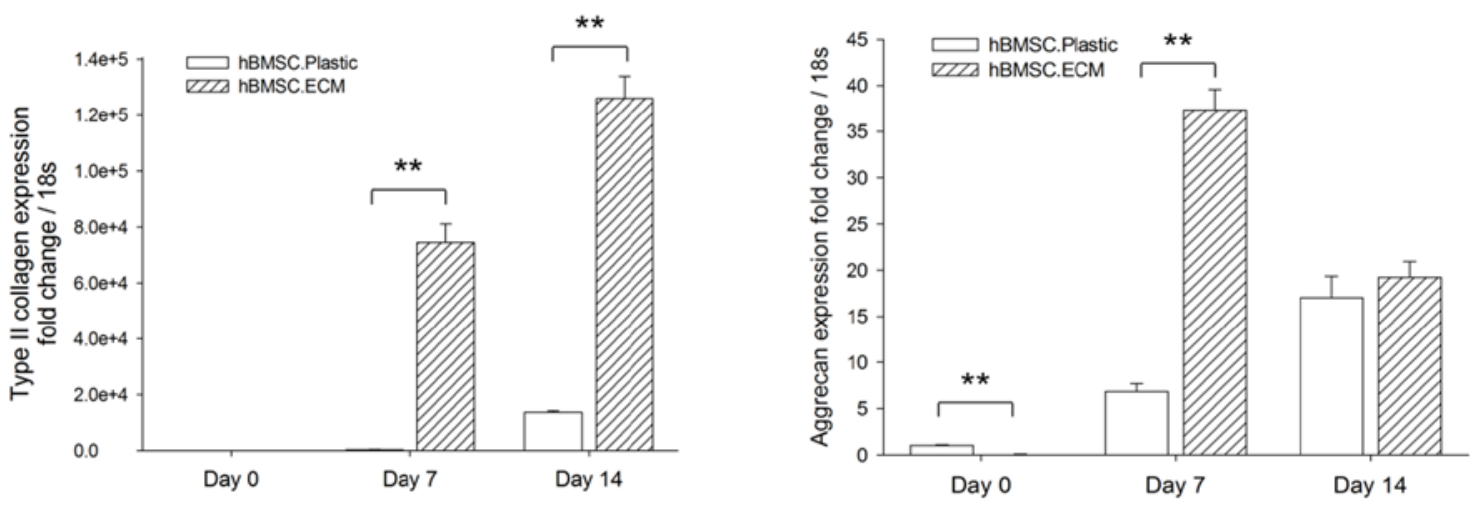

C
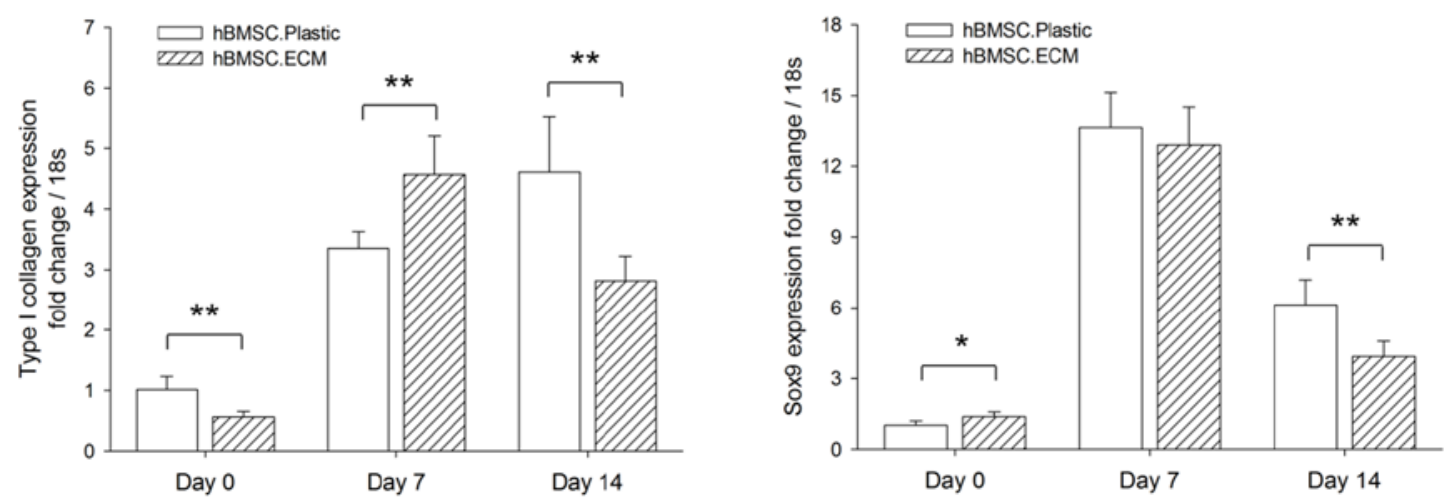
Figure 6.7

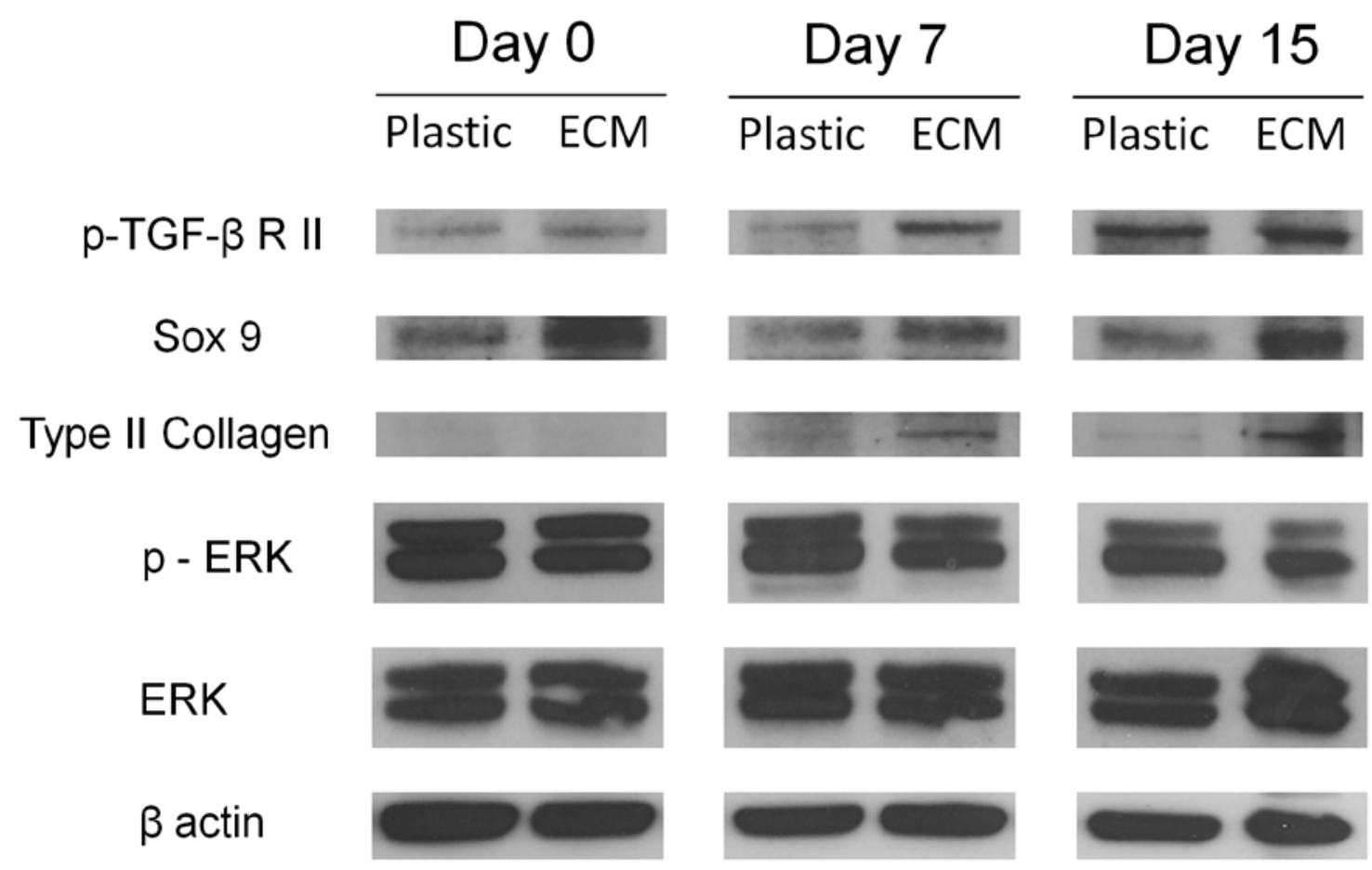




\section{Chapter 7}

\section{Comparison of three-dimensional extracellular matrices deposited by synovium-derived stem cells or articular chondrocytes}

Superiority of SDSC-deposited ECM as a universal novel expansion system in vitro to promote cell self-renewal and chondrogenic potential

Fan He, B.S. and Ming Pei, M.D., Ph.D.

Stem Cell and Tissue Engineering Laboratory, Department of Orthopaedics and Division of Exercise Physiology, West Virginia University, Morgantown, West Virginia 26506

Key words: Synovium, Chondrocyte, Extracellular matrix, Chondrogenesis, Cartilage regeneration, TGF- $\beta$ 


\begin{abstract}
Three-dimensional extracellular matrix (ECM) has been used as an in vitro expansion system for mesenchymal stem cells. However it is unknown whether different ECMs distinctly affect cell proliferation and differentiation. We generated two types of ECM separately from synovium-derived stem cells (SDSCs) and articular chondrocytes. SDSC-deposited ECM (S-ECM) contains fibrillar type I collagen whereas chondrocyte-deposited ECM (C-ECM) composes fibrillar type I and II collagens with proteoglycans. SDSCs and chondrocytes were separately expanded on three substrates: conventional plastic culture flasks (Plastic), S-ECM and C-ECM. The cell proliferative rate was improved by the two ECMs compared with the conventional plastic culture system. S-ECM promoted cell self-renewal of SDSCs and chondrocytes but C-ECM showed strong support on chondrocytes only. SDSC and chondrocyte pellets were induced to chondrogenic differentiation with transforming growth factor $\beta$-1. The chondrogenic potential of SDSCs and chondrocytes after expansion on ECM was enhanced. However SDSCs expanded on S-ECM expressed a higher level of chondrogenic genes and generated more cartilage-specific matrix than those on C-ECM. Chondrocytes expanded on S-ECM and C-ECM showed a similar chondrogenic differentiation level. The enhanced chondrogenic potential by 3D ECM is partially related to the high sensitivity of TGF- $\beta$ receptor II. In conclusion, we demonstrated that SDSC-deposited ECM was superior to chondrocyte-deposited ECM and functions as a universal expansion system in vitro for MSCs and differentiated cells to promote proliferative and chondrogenic potentials.
\end{abstract}




\section{INTRODUCTION}

Articular cartilage is a unique tissue with an avascular structure. ${ }^{1}$ Due to the limited self-repair mechanism, cartilage is vulnerable to degenerative disease after trauma or osteoarthritis. ${ }^{2 ; 3}$ Autologous chondrocyte transplantation (ACT) has been successfully used in clinical practice to repair cartilage defects. ${ }^{4 ; 5} \mathrm{ACT}$ relies on a biopsy from a non-weight bearing cartilage area, expansion of articular chondrocytes in vitro, and transplantation into the defect site which is sealed with a periosteal graft or collagen membrane. ${ }^{6 ; 7}$ The disadvantages of ACT includes the rapid dedifferentiation of chondrocytes during in vitro expansion, which is the process of cell morphology changing to a fibroblast-like shape and an elevated expression of type I collagen instead of type II collagen. ${ }^{5-7}$

Adult mesenchymal stem cells (MSCs) provide a promising approach for the cartilage regeneration; these cells are derived from various tissues including bone marrow, ${ }^{8 ; 9}$ adipose tissue,${ }^{10}$ synovium, ${ }^{11}$ periosteum, ${ }^{12}$ skeletal muscle $^{13}$ and other connective tissues ${ }^{14}$. Numerous researchers have reported the successful generation of hyaline cartilage-like tissue in vitro ${ }^{15 ; 16}$ and the use of this tissue to repair partial-thickness or full-thickness cartilage defects in animal models. ${ }^{17 ; 18}$ Among the various MSCs, synovium-derived stem cells (SDSCs) are considered to have superior proliferative ability and chondrogenic potential. ${ }^{19 ; 20}$

MSC-based cartilage regeneration, however, faces similar challenges as ACT. The limited self-renewal ability of MSCs is one of disadvantages compared with embryonic stem cells (ESCs) and induced pluripotent stem (iPS) cells. ${ }^{21 ; 22}$ The proliferative ability of MSCs usually dramatically decreases after 10 passages and eventually disappears. ${ }^{23 ; 24}$ To retain the proliferative ability of MSCs, cells are required to be cultured from a relatively low seeding density $^{25 ; 26}$ or stimulated with growth factors such as fibroblast growth factor 2 (FGF-2). ${ }^{27}$ The conventional in vitro culture of MSCs requires weekly passage in order to maintain their proliferative and multipotential abilities. ${ }^{28}$ However MSCs of late passages ( $\left.\geq 15\right)$ still lose their multipotency and only differentiate to adipocytes. ${ }^{29}$ The limitation of the current in vitro culture 
of MSCs is that, once isolated from the in vivo microenvironment, they lose their proliferation and multipotent differentiation potentials similar to aged cells. ${ }^{30-32}$

Our previous work demonstrated that the utilization of a natural, three-dimensional (3D), cell-deposited extracellular matrix $(\mathrm{ECM})^{33}$ in the expansion of SDSCs can dramatically improve SDSC self-renewal and chondrogenic potential. This native 3D ECM has also been deployed in the culture of articular chondrocytes that successfully delayed chondrocyte dedifferentiation and enhanced redifferentiation. However this proposed another question whether tissue-specific generated ECM would have a similar or opposite effect on cell proliferation and differentiation.

Type I collagen was confirmed to be one of the components in SDSC-deposited $\mathrm{ECM}^{33}$ that has been used to promote proliferation and differentiation of MSCs and articular chondrocytes in two-dimensional (2D) substrate ${ }^{34}$ and 3D gel format. ${ }^{35}$ Type II collagen is another candidate to improve cell attachment and chondrogenesis; it is less commonly used than type I collagen. Hyaline cartilage is composed of type II collagen and sulfated proteoglycans (mainly aggrecan) that support chondrocyte morphology and gene expressions. ${ }^{36}$ Therefore, chondrocytes or MSCs in scaffolds made by type II collagen coated with proteoglycans have a better potential to promote cell proliferative rate and chondrogenic differentiation potential than type I collagen gel. ${ }^{37 ; 38}$

In the current study, we hypothesized that ECM deposited by different cells can influence cell proliferation and differentiation potentials in distinct ways. We manufactured two distinct ECMs deposited by SDSCs or articular chondrocytes. Porcine SDSCs and chondrocytes were separately expanded under three conditions: the conventional plastic culture flasks (Plastic) serving as the control group and SDSC-deposited ECM (S-ECM) and chondrocyte-deposited ECM (C-ECM) serving as experimental groups. The data showed that, when culturing SDSCS, S-ECM showed superiority in cell proliferation and chondrogenesis over C-ECM and Plastic. Surprisingly, we found that chondrocytes expanded on S-ECM and C-ECM showed similar improvement that was much greater than cells grown on Plastic. 


\section{MATERIALS and METHODS}

Isolation and culture of SDSCS and chondrocytes

Synovium and cartilage tissue were collected from two 3-month-old miniature pigs as previously described. ${ }^{20 ; 39}$ Briefly, chondrocytes were digested with $0.2 \%$ collagenase $P$ (Roche, Indianapolis, IN) at $37^{\circ} \mathrm{C}$ overnight after being finely minced into pieces with a scalpel. Synovium membrane was digested at $37^{\circ} \mathrm{C}$ for 30 minutes in $0.1 \%$ trypsin (Roche) and then for 2 hours in $0.1 \%$ collagenase $\mathrm{P}$ to release SDSCs. Chondrocytes and SDSCs were resuspended and plated in culture medium [ $\alpha$-minimum essential medium (Invitrogen, Carlsbad, CA) containing 10\% fetal bovine serum (FBS, Atlanta Biologicals, Lawrenceville, GA), $100 \mathrm{U} / \mathrm{ml}$ penicillin, $100 \mu \mathrm{g} / \mathrm{ml}$ streptomycin and $0.25 \mu \mathrm{g} / \mathrm{ml}$ fungizone (Invitrogen)] at $37^{\circ} \mathrm{C}$ in a humidified atmosphere of $5 \%$ carbon dioxide and $95 \%$ air. Non-adherent cells were removed by medium change every three days. For negative isolation of SDSCs from primary cultures, cell suspension was incubated with Dynabeads ${ }^{\circledR}$ M-450 CD14 conjugated with a monoclonal antibody specific for macrophages (Dynal Biotech, Oslo, Norway) at $4^{\circ} \mathrm{C}$ for 1 hour. The conjugated cells and the unbound Dynabeads ${ }^{\circledR}$ were collected by using the Dynal Magnetic Particle Concentrator ${ }^{\circledR}$ (Dynal Biotech) and the depleted supernatant with SDSCs was saved for further passaging.

\section{Preparation of SDSC-ECM (S-ECM) and Chondrocyte-ECM (C-ECM)}

The procedure of obtaining cell-free extracellular matrix was described previously ${ }^{33} .75 \mathrm{~cm}^{2}$ plastic flasks were pretreated with $0.2 \%$ gelatin solution (Sigma, St. Louis, MO) for 1 hour at $37^{\circ} \mathrm{C}$, followed by $1 \%$ glutaraldehyde (Sigma) and $1 \mathrm{M}$ ethanolamine (Sigma) for 30 minutes separately at room temperature. SDSCs were seeded on pretreated plastic flasks at the density of 3,000 cells $/ \mathrm{cm}^{2}$ in culture medium for 7 days. After reaching $90 \%$ confluence, 50 $\mathrm{mM}$ of L-ascorbic acid phosphate (Wako, Richmond, VA) was added and culturing was continued for an additional 8 days. Chondrocytes were seeded on pretreated plastic flasks at the density of 3,000 cells $/ \mathrm{cm}^{2}$ to reach $90 \%$ confluence and changed to complete medium [high-glucose DMEM (Cellgro, Herdon, VA), 10\% FBS, $100 \mathrm{U} / \mathrm{ml}$ penicillin, $100 \mu \mathrm{g} / \mathrm{ml}$ streptomycin, 10 mM HEPES (Sigma), 0.1 mM non-essential amino acids (Sigma), 0.4 mM 
proline (Sigma), and $50 \mathrm{mg} / \mathrm{L}$ l-ascorbic acid] for an additional 8 days under $5 \%$ oxygen. To obtain cell-free ECM, S-ECM and C-ECM were incubated with 0.5\% Triton X-100 (Sigma) containing $20 \mathrm{mM}$ ammonium hydroxide (Sargent-Welch, Skokie, IL) for 5 minutes at $37^{\circ} \mathrm{C}$, followed by $100 \mathrm{u} / \mathrm{mL}$ DNase I (Sigma) for 1 hour at $37^{\circ} \mathrm{C}$ and stored at $4^{\circ} \mathrm{C}$.

In vitro culture of SDSCS and chondrocytes on Plastic and ECM

SDSCs were expanded from passage 4 to passage 6 under three conditions: plastic flasks (Plastic), flasks coated with SDSC-deposited ECM (S-ECM), and flasks coated with chondrocyte-deposited ECM (C-ECM). Chondrocytes were cultured similarly to SDSCs for 3 further passages. The culture period of each passage was 7 days and the medium was changed every 3 days. At each passage, cell number was calculated by a counting hemacytometer (Hausser Scientific, Horsham, PA) for cell proliferative rate.

In vitro chondrogenic differentiation of SDSCs and chondrocytes

$3 \times 10^{5}$ of SDSCs and chondrocytes of passage 6 cultured on Plastic, S-ECM and C-ECM were centrifuged to form pellets in a 15-ml polypropylene tube at $500 \mathrm{~g}$ for 5 minutes. After 24 hours, the pellets (Day 0) were incubated in chondrogenic medium [high-glucose DMEM, 40 $\mu \mathrm{g} / \mathrm{ml}$ proline, $100 \mathrm{nM}$ dexamethasone (Sigma), $100 \mathrm{U} / \mathrm{ml}$ penicillin, $100 \mu \mathrm{g} / \mathrm{ml}$ streptomycin, $0.1 \mathrm{mM}$ ascorbic acid-2-phosphate and ITS ${ }^{\mathrm{TM}}$ Premix (BD Biosciences, Bedford, MA) with the supplementation of $10 \mathrm{ng} / \mathrm{ml}$ of TGF- $\beta 1$ (Pepro Tech Inc., Rocky Hill, NJ)]. Pellets at Day 0, Day 7 and Day 14 were collected for further analysis.

\section{Histology and immunohistochemistry}

The ECM deposited by SDSCs and chondrocytes and the chondrogenic pellets $(n=2)$ were fixed in $4 \%$ paraformaldehyde, embedded in paraffin blocks and cut into $5-\mu \mathrm{m}$ thick sections. To detect glycosaminoglycans, sections were stained with $0.2 \%$ Alcian blue solution (Sigma). For immunohistochemistry, the sections were incubated with primary antibodies against type II collagen (II-II6B3, Developmental Studies Hybridoma Bank DSHB, lowa City, IA), type I collagen (Sigma) and type $X$ collagen (Sigma), followed by the secondary antibody of biotinylated horse anti-mouse IgG (Vector, Burlingame, CA) and detected by using Vectastain 
$A B C$ reagent (Vector) with 3,3'-diaminobenzidine (DAB) as a substrate.

Biochemical analysis for DNA and glycosaminoglycan (GAG) content To examine DNA and glycosaminoglycan content in pellets, samples $(n=4)$ were digested for 6 hours at $60^{\circ} \mathrm{C}$ by $125 \mu \mathrm{g} / \mathrm{ml}$ papain in PBE buffer ( $100 \mathrm{mM}$ phosphate, $10 \mathrm{mM}$ EDTA, pH 6.5) containing $10 \mathrm{mmol} / \mathrm{L}$ cysteine. The amount of DNA was measured by QuantiT ${ }^{\mathrm{TM}}$ PicoGreen $^{\circledR}$ dsDNA assay kit (Invitrogen) and CytoFluor ${ }^{\circledR}$ Series 4000 (Applied Biosystems, Foster City, CA) following by the protocol. The GAG content was measured by using $16 \mu \mathrm{g} / \mathrm{ml}$ of dimethylmethylene blue dye and a Spectronic ${ }^{\mathrm{TM}}$ BioMate $^{\mathrm{TM}} 3$ Spectrophotometer (Thermo Scientific, Milford, MA) with bovine chondroitin sulfate (Sigma) as the standard.

\section{Real-time polymerase chain reaction (Real-time PCR)}

The total mRNA from pellets $(n=4)$ was extracted using TRIzol ${ }^{\circledR}$ (Invitrogen) and converted to cDNA with High-Capacity cDNA Archive Kit as recommended by the manufacturer (Applied Biosystems). Chondrogenic marker genes (aggrecan, type I and II collagen, and Sox9) and hypertrophy marker genes (type X collagen, MMP13) were customized by Applied Biosystem as part of the Custom $\operatorname{Taqman}^{\circledR}$ Gene. Expression Assays Eukaryotic 18S rRNA (Assay ID HS99999901_S1 ABI) was carried out as the endogenous control gene. Real-time PCR was performed with the iCycler $\mathrm{iQ}^{\mathrm{TM}}$ Mutil Color RT-PCR Detection and calculated by computer software (Perkin-Elmer, Waltham, MA). Relative transcript levels were calculated as $X=2^{-\Delta \Delta C t}$, in which $\Delta \Delta \mathrm{Ct}=\Delta \mathrm{E}-\Delta \mathrm{C}, \Delta \mathrm{E}=\mathrm{Ct}_{\exp }-\mathrm{Ct}_{18 \mathrm{~s}}$, and $\Delta \mathrm{C}=\mathrm{Ct}_{\mathrm{ct1}}-\mathrm{Ct}_{18 \mathrm{~s}}$.

\section{Immunoblotting}

The cytoplasm protein of pellets was extracted by using RIPA lysis buffer (Thermo Scientific) supplemented with halt protease and phosphatase inhibitor cocktail (Thermo Scientific). The protein concentration was quantified using the $\mathrm{BCA}^{\mathrm{TM}}$ Protein Assay Kit (Thermo Scientific). The samples was denatured and separated by NuPAGE ${ }^{\circledR}$ Novex $^{\circledR}$ Bis-Tris Mini Gels (Invitrogen) at $200 \mathrm{~V}$ for 45 minutes at $4^{\circ} \mathrm{C}$, then transferred onto a nitrocellulose membrane (Invitrogen) using $\mathrm{XCell} \mathrm{II}^{\mathrm{TM}}$ blot module (Invitrogen) at $30 \mathrm{~V}$ for 1 hour at $4^{\circ} \mathrm{C}$. The membranes were first blocked in SuperBlock (TBS) Blocking Buffer (Thermo Scientific), then 
incubated with the primary monoclonal antibodies of phospho-TGF $\beta$ RII (Tyr 424) (Santa Cruz Biotechnology, Santa Cruz, CA), Sox9 (Abcam, Cambridge, MA), type II collagen (II-II6B3), phospho-p44/42 MAPK (Erk1/2) (Thr202/Tyr204) (Cell Signaling Technology, Danvers, MA), p44/42 MAPK (Erk1/2) (Cell Signaling) and $\beta$-actin (Abcam) at the recommended dilution overnight at $4^{\circ} \mathrm{C}$. The membranes were incubated in the secondary antibody of Goat Anti-Mouse IgG $(\mathrm{H}+\mathrm{L})$ or Goat Anti-Rabbit IgG $(\mathrm{H}+\mathrm{L})$ (Thermo Scientific) for 40 minutes at room temperature and exposured using SuperSignal ${ }^{\circledR}$ West Pico Chemiluminescent Substrate (Thermo Scientific) and CL-XPosure ${ }^{\mathrm{TM}}$ Film (Thermo Scientific).

\section{Statistical Analysis}

The Kruskal-Wallis test was used to test for significant differences among all groups and the Mann-Whitney $U$ test was used for pairwise comparison. All statistical analyses were performed with SPSS 13.0 statistical software (SPSS Inc., Chicago, IL). P values less than 0.05 were considered statistically significant. 


\section{RESULTS}

Immunohistochemistry of SDSC-deposited ECM and chondrocyte-deposited ECM

S-ECM showed strong expression of type I collagen but absence of sulfated GAGs or type II collagen. The composition of S-ECM was consistent with previous studies on ECM derived from bone marrow stem cells and fibroblasts. ${ }^{33 ; 40}$ The components of C-ECM were revealed to be more complex than S-ECM, which contained sulfated GAGs, type I and II collagen.(Figure 7.1).

In vitro expansion of SDSCS and chondrocytes on Plastic, S-ECM and C-ECM

The cell morphology of SDSCs and chondrocytes on Plastic is flat and large, but when cells were cultured on S-ECM and C-ECM, they appeared spindle-like and small. The morphologic appearance of S-ECM and C-ECM was unchanged within 3 passages. Moreover, cell migration was directional on S-ECM and C-ECM compared to the random movement on Plastic (Figure 7.1B).

The proliferative rate of SDSCs on S-ECM had a 2.4- to 7.2- fold increase compared to cells on Plastic and average 2-fold higher than cells on C-ECM (Figure 7.1C). At passage 4 chondrocytes on S-ECM and C-ECM yielded a similar cell number that was 6-fold that of Plastic. At passage 5 and 6, chondrocytes on S-ECM exhibited 20\%-40\% higher proliferative ability than that on C-ECM, and maintained 4- to 12-fold that on Plastic (Figure 7.1D). Cells on S-ECM regained the highest proliferative rate; the proliferation ability was improved on C-ECM but still lower than S-ECM and both S-ECM and C-ECM were higher than Plastic.

\section{S-ECM mostly improved chondrogenic differentiation potential of SDSCS}

Passage 6 SDSCs expanded on Plastic, S-ECM and C-ECM were centrifuged to form pellets and induced to chondrogenic differentiation by TGF- $\beta 1$. SDSCs of S-ECM group showed strong expression of sulfated GAGs stained by Alcian blue, and extensive expression of type I and II collagen. SDSCs of C-ECM group were positive for Alcian blue staining; immunohistochemistry showed type I and II collagens at Day 14 but the results were weaker 
than S-ECM group. SDSCs of Plastic group had the worst chondrogenic differentiation potential as proven by the partial positive staining at Day 14 (Figure 7.2).

At Day 14 pellets of S-ECM group generated $23.5 \pm 1.6 \mu \mathrm{g}$ of sulfated GAGs compared to $11.1 \pm 1.6 \mu \mathrm{g}$ in C-ECM group and $2.5 \pm 0.8 \mu \mathrm{g}$ in Plastic group (Figure 7.3A). The DNA content was maintained in S-ECM group; it was 81\% at Day 14 which is higher than that of C-ECM (45\%) and Plastic groups (21\%) (Figure 7.3B). The chondrogenic index, defined by the ratio of sulfated GAGs to DNA, had the highest level in S-ECM group (39.3 \pm 3.1 at Day 14), with lower level in C-ECM $(27.8 \pm 4.4)$ and Plastic groups (7.6 \pm 2.2$)$ (Figure 7.3C).

The expression of chondrogenic and hypertrophic genes was quantified by real-time PCR. The level of type II collagen mRNA in S-ECM was 464-fold and 4-fold that of Plastic group and C-ECM group at Day 7, respectively (Figure 7.3D). The expression of aggrecan mRNA was highest in S-ECM; it was 11-fold and 2-fold that of Plastic and C-ECM at Day 7, respectively (Figure 7.3E). The expression of type I collagen mRNA was similar in S-ECM and C-ECM groups and was at least 30\% higher than that of Plastic group (Figure 7.3F). Sox-9, the transcription factor in chondrogenesis, mRNA expression was highest in S-ECM group (Figure 7.3G). During chondrogenesis the level of type $X$ collagen mRNA was not significantly different between the three groups (Figure $7.3 \mathrm{H}$ ), but the level of MMP13 mRNA was highest in S-ECM group at Day 7 and higher in S-ECM and C-ECM than Plastic group (Figure 7.3I).

\section{S-ECM and C-ECM elevated the activity of receptors of TGF- $\beta$ in SDSCS}

During the induction of chondrogenesis, immunoblotting showed that the TGF- $\beta$ receptor II (TGF- $\beta$ RII) in the three groups was activated but the activity of TGF- $\beta$ RII was higher in S-ECM and C-ECM groups than that in Plastic group. Correspondingly, the expressions of Sox9 and type II collagen were stronger in S-ECM and C-ECM groups than in Plastic group. However, ERK1/2 activity was suppressed during chondrogenesis except the high phosphorylation level of C-ECM group at Day 14 (Figure 7.4). 
The chondrogenic potential of chondrocytes was improved by S-ECM and C-ECM

After expansion on Plastic, S-ECM and C-ECM, chondrocytes of passage 6 were induced to chondrogenesis in the pellet culture system. At Day 7 and 14, chondrocyte pellets of S-ECM and C-ECM groups showed stronger expression of sulfated GAGs, type I and II collagens than those of Plastic group. The chondrogenic differentiation potential was improved in chondrocytes after S-ECM and C-ECM expansion (Figure 7.5).

To compare the chondrogenic differentiation effect, biochemistry was used to quantify the content of sulfated GAGs and DNA in each group. At Day 14 chondrocyte pellets generated high amount of sulfated GAGs in S-ECM and C-ECM groups at the similar level $(58.1 \pm 2.5$ $\mu \mathrm{g}$ vs. $56.1 \pm 3.0 \mu \mathrm{g}$ ), but the level of GAGs in Plastic group was only $47 \%$ of that in ECM groups $(27.2 \pm 1.6 \mu \mathrm{g})$ (Figure 7.6A). At Day 14 the DNA content of chondrocyte pellets in Plastic group dropped to $35 \%$ but increased to $111 \%$ in S-ECM group and $119 \%$ in C-ECM group (Figure 7.6B). The chondrogenic index in the three groups at Day 14 was relatively high and that of Plastic group reached $92.7 \pm 3.0$. The chondrogenic index of C-ECM group was $8.3 \%$ higher than that of S-ECM $(105.5 \pm 4.9$ vs. $97.5 \pm 5.2)$ and both were higher than Plastic group (Figure 7.6C).

At the level of mRNA expression chondrocyte pellets of S-ECM and C-ECM groups had a higher level than those of Plastic group in chondrogenic and hypertrophic genes (Figure 7.6 D-I). But the difference between S-ECM and C-ECM was not significant. At the level of type II collagen and aggrecan mRNA, pellets of S-ECM and C-ECM groups expressed 5-fold and 7-fold, respectively, as that of Plastic group (Figure 7.7 D\&E). At Day 7 the expression of type $X$ collagen in S-ECM and C-ECM groups was $80 \%$ higher than that of Plastic group (Figure 7.6H). At Day 14 the level of MMP13 mRNA in S-ECM and C-ECM groups was 6.2-fold that of Plastic group (Figure 7.6I).

\section{S-ECM and C-ECM improved the activity of receptors of TGF- $\beta$ in chondrocytes}

Before treatment with TGF- $\beta 1$, the level of phosphorylated TGF- $\beta$ RII in Plastic group was much higher than that in ECM groups. After the chondrogenic induction the activity of TGF- $\beta$ 
RII in S-ECM and C-ECM groups was higher than that of Plastic group. The expression of Sox9 and type II collagen in S-ECM and C-ECM groups was stronger than that of Plastic group. The phosphorylation of ERK1/2 was decreased after expansion on S-ECM and C-ECM and the activity was suppressed during chondrogenic differentiation (Figure 7.7). 


\section{DISCUSSION}

In this study we used porcine SDSCs to deposit S-ECM composed of a fibrillar type I collagen network and articular chondrocytes to generate C-ECM that contained a type I and II collagen network with proteoglycans. Though the two types of ECMs had three-dimensional structure and were deprived of the original feeder cells, they showed a distinct influence on cell proliferative rate and chondrogenic potential. SDSC-deposited ECM improved the proliferation rate and chondrogenic differentiation of both SDSCs and chondrocytes, while chondrocyte-deposited ECM only significantly affected chondrocyte expansion rate and chondrogenesis. However both S-ECM and C-ECM promoted cell self-renewal and chondrogenic potential compared to the conventional plastic culture system.

The traditional 2D reconstructed ECM uses monomeric proteins to coat substrates such as fibronectin, laminin, gelatin, and denatured collagens, to improve MSC proliferation and differentiation. However, the 3D culture system has been proven more efficient than 2D culture substrates in cell expansion and preserving the multipotent capability for MSCs in vitro. The 3D natural cell-deposited ECM used in this study has been adopted to study the signaling pathway in cell adhesion ${ }^{41}$ and cell invasion. ${ }^{42 ; 43}$ Recent studies showed that the utilization of 3D ECM in expansion of MSCs in vitro can greatly improved cell proliferation and enhanced multipotential differentiation..$^{33 ; 40 ; 44}$ Not only MSCs but also terminally differentiated cells such as adult articular chondrocytes and nucleus pulposus cells exhibited enhanced self-renewal and redifferentiation potential after expansion on 3D ECM. However it is important to establish a tissue-specific microenvironment in vitro to direct MSC differentiation. Due to the similar properties shared by MSCs from many sources and the same procedure to generate ECM, MSC-deposited ECMs are identical in structure and composition. ${ }^{33 ; 40 ; 41 ; 45}$ We chose articular chondrocytes to reconstruct the native 3D ECM that mimics hyaline cartilage tissue. The component of fibrillar type II collagen associated with proteoglycans in chondrocyte-deposited ECM was one of the major differences from SDSC-deposited ECM. The presence of type I collagen in C-ECM was partially due to the dedifferentiation of articular chondrocytes in the in vitro culture. ${ }^{5 ; 7}$ 
Type I collagen was used as a substrate and gel to facilitate cell expandability and differentiation and achieved better results than other ECM proteins. Compared to type I collagen, type II collagen was less commonly used for cell expansion but was proven to induce chondrocyte redifferentiation more effectively. Bosnakovski et al. provided evidence that bone marrow stem cells seeded in type II collagen hydrogel generated more cartilage-specific genes and matrix than cells in type I collagen gel. ${ }^{46}$ Moreover, Buma et al. reported that using type II collagen scaffold to repair full-thickness articular cartilage defects generated hyaline cartilage-like tissue instead of fibro-cartilaginous tissue by type I collagen. ${ }^{47}$ Moreover, proteoglycans were found in C-ECM rather than S-ECM. Previous studies showed that type II collagen-GAG scaffolds were able to enhance cell chondrogenesis $^{48}$ and had more stiffness than a type I collagen scaffold. ${ }^{49}$ Besides the different composition, the rigidity of the two ECMs caused distinct proliferation and differentiation potentials in SDSCs and chondrocytes. Winer et al. compared the effect of substrates with different rigidity on human BMSCs and demonstrated that cells became quiescent on the soft matrix (250 Pa) but resumed the proliferation when transferred to a stiff substrate. $^{50}$

The great improvement on cell proliferative rate after culturing on the two types of ECM was partially induced by the activation of the integrin/Src/ERK1/2 signaling pathway and the activation of cyclin D1. ${ }^{51-53}$ However, the different proliferation rates of SDSCs on the two ECMs suggested that the signaling pathway may not be exactly the same. Cells recognize fibrillar collagens mainly through integrin $\alpha_{2} \beta_{1}$ and $\alpha_{5} \beta_{1}{ }^{54-57}$ but recently it was reported that Rock 2 expression was suppressed in type II collagen-rich gel instead of type I collage gel. ${ }^{58}$ Besides the increased proliferative rate, SDSCs and chondrocytes showed directional migration along the fibrils of ECM rather than the random migration found on Plastic. One possible explanation is that ECM can effectively reduce the activity of Rac that is responsible for the random cell migration..$^{59-61}$

Induced by TGF- $\beta$, SDSCs showed higher chondrogenic differentiation potential after 
expansion on S-ECM and chondrocytes cultured on S-ECM and C-ECM showed a similar level of chondrogenic genes and matrix. The enhanced chondrogenic potential caused by ECM is explained by the high activity of TGF- $\beta$ RII when induced with TGF- $\beta$. More interestingly, chondrocytes cultured on Plastic had a highly phosphorylated level of TGF- $\beta$ RII before chondrogenesis that was possibly autophosphorylation and induced spontaneous differentiation in the monolayer culture period. ${ }^{40 ; 62 ; 63}$ The spontaneous differentiation was reported by Chen et al. and it suppressed the later growth factor-induced specific differentiation. ${ }^{40} \mathrm{ECM}$ significantly reduced the degree of autophosphorylation, increased the sensitivity of TGF- $\beta$ RII and enhanced the effectiveness of TGF- $\beta$ induction to chondrogenesis. Among the various ECM proteins, decorin may play an important role in preventing TGF- $\beta$ receptor autophosphorylation that was proven to exist in BMSC-deposited $\mathrm{ECM}^{40}$ Decorin, often found in type I collagen-rich matrices and composed of a leucine repeated core protein attached with a glycosaminoglycan chain of varying length and composition, is considered as the antagonist of TGF- $\beta$, masks the binding site of TGF- $\beta$ receptors $^{64 ; 65}$ and contributes to the low level of autophosphorylation of TGF- $\beta$ receptor.

The MAPK signaling pathway was previously considered to promote chondrogenic transcription factors and induce the expression of chondrogenic genes. ${ }^{66 ; 67}$ Recently several studies suggested that the inhibition of the MAPK signaling pathway can improve MSC chondrogenic differentiation. ${ }^{68-70}$ Consistent with our immunoblotting data, ERK1/2 activity was suppressed during chondrogenesis and was significantly lower in SDSCs and chondrocytes expanded on ECM than those expanded on Plastic. The robust elevation of phosphorylated ERK1/2 at Day 14 in SDSCs of C-ECM and chondrocytes in S-ECM is possibly related to the increased expression of hypertrophic genes. ${ }^{71}$ Type $X$ collagen is the well-accepted hypertrophic marker and in late chondrogenesis MMP13 and ALP are proposed as other markers for hypertrophy. ${ }^{72 ; 73}$ The increase of hypertrophic genes after ECM expansion is not consistent with our previous study ${ }^{33}$ and due to the complex mechanism of chondrocyte hypertrophic differentiation, future studies will be conducted on the hypertrophic issue by applying different growth factors in vitro and conducting transplantation in vivo. 
In summary, we compared the influence of two natural 3D extracellular matrices, deposited by SDSCs and chondrocytes, on cell proliferation and chondrogenic potential. SDSC-deposited ECM can improve the proliferative and chondrogenic capacities of SDSCS and chondrocytes. Chondrocyte-deposited ECM can only promote chondrocyte proliferation and chondrogenesis. The enhanced chondrogenic potential of cells expanded on 3D ECM is related to the high sensitivity of TGF- $\beta$ receptor II. Together with previous studies, we demonstrate that SDSC-deposited extracellular matrix functions as a universal expansion system in vitro for MSCs and differentiated cells to promote proliferative and chondrogenic potentials. 


\section{REFERENCES}

1. Muir H. The chondrocyte, architect of cartilage. Biomechanics, structure, function and molecular biology of cartilage matrix macromolecules. Bioessays 1995;17:1039-48.

2. Beris $A E$, Lykissas MG, Papageorgiou $C D$ et al. Advances in articular cartilage repair. Injury 2005;36 Suppl 4:S14-S23.

3. Mollenhauer JA. Perspectives on articular cartilage biology and osteoarthritis. Injury 2008;39 Suppl 1:S5-12.

4. Brittberg M, Lindahl A, Nilsson A et al. Treatment of deep cartilage defects in the knee with autologous chondrocyte transplantation. N.Engl.J.Med. 1994;331:889-95.

5. Lin L, Zhou C, Wei X et al. Articular cartilage repair using dedifferentiated articular chondrocytes and bone morphogenetic protein 4 in a rabbit model of articular cartilage defects. Arthritis Rheum. 2008;58:1067-75.

6. Dell'Accio F, Vanlauwe J, Bellemans $\mathrm{J}$ et al. Expanded phenotypically stable chondrocytes persist in the repair tissue and contribute to cartilage matrix formation and structural integration in a goat model of autologous chondrocyte implantation. J.Orthop.Res. 2003;21:123-31.

7. Marlovits $S$, Zeller $\mathrm{P}$, Singer $\mathrm{P}$ et al. Cartilage repair: generations of autologous chondrocyte transplantation. Eur.J.Radiol. 2006;57:24-31.

8. Piersma $\mathrm{AH}$, Brockbank KG, Ploemacher RE et al. Characterization of fibroblastic stromal cells from murine bone marrow. Exp. Hematol. 1985;13:237-43.

9. Pittenger MF, Mackay AM, Beck SC et al. Multilineage potential of adult human mesenchymal stem cells. Science 1999;284:143-7.

10. Zuk PA, Zhu M, Ashjian P et al. Human adipose tissue is a source of multipotent stem cells. Mol.Biol.Cell 2002;13:4279-95.

11. De BC, Dell'Accio F, Tylzanowski P et al. Multipotent mesenchymal stem cells from adult human synovial membrane. Arthritis Rheum. 2001;44:1928-42.

12. De BC, Dell'Accio F, Luyten FP. Human periosteum-derived cells maintain phenotypic stability and chondrogenic potential throughout expansion regardless of donor age. Arthritis Rheum. 2001;44:85-95. 
13. Asakura A, Komaki M, Rudnicki M. Muscle satellite cells are multipotential stem cells that exhibit myogenic, osteogenic, and adipogenic differentiation. Differentiation 2001;68:245-53.

14. Krampera M, Pizzolo G, Aprili G et al. Mesenchymal stem cells for bone, cartilage, tendon and skeletal muscle repair. Bone 2006;39:678-83.

15. Kuo CK, Li WJ, Mauck RL et al. Cartilage tissue engineering: its potential and uses. Curr.Opin.Rheumatol. 2006;18:64-73.

16. Pei M, He F, Kish VL et al. Engineering of functional cartilage tissue using stem cells from synovial lining: a preliminary study. Clin.Orthop.Relat Res. 2008;466:1880-9.

17. Pei M, He F, Boyce BM et al. Repair of full-thickness femoral condyle cartilage defects using allogeneic synovial cell-engineered tissue constructs. Osteoarthritis.Cartilage. 2009;17:714-22.

18. Gelse K, Muhle C, Franke O et al. Cell-based resurfacing of large cartilage defects: long-term evaluation of grafts from autologous transgene-activated periosteal cells in a porcine model of osteoarthritis. Arthritis Rheum. 2008;58:475-88.

19. Sakaguchi $\mathrm{Y}$, Sekiya I, Yagishita $\mathrm{K}$ et al. Comparison of human stem cells derived from various mesenchymal tissues: superiority of synovium as a cell source. Arthritis Rheum. 2005;52:2521-9.

20. Pei M, He F, Vunjak-Novakovic G. Synovium-derived stem cell-based chondrogenesis. Differentiation 2008;76:1044-56.

21. Jukes JM, Both SK, Leusink A et al. Endochondral bone tissue engineering using embryonic stem cells. Proc.Natl.Acad.Sci.U.S.A 2008;105:6840-5.

22. Yu J, Vodyanik MA, Smuga-Otto $\mathrm{K}$ et al. Induced pluripotent stem cell lines derived from human somatic cells. Science 2007;318:1917-20.

23. Koga $\mathrm{H}$, Muneta $\mathrm{T}$, Nagase $\mathrm{T}$ et al. Comparison of mesenchymal tissues-derived stem cells for in vivo chondrogenesis: suitable conditions for cell therapy of cartilage defects in rabbit. Cell Tissue Res. 2008;333:207-15.

24. Yoshimura $H$, Muneta $T$, Nimura $A$ et al. Comparison of rat mesenchymal stem cells derived from bone marrow, synovium, periosteum, adipose tissue, and muscle. Cell Tissue Res. 2007;327:449-62. 
25. Sekiya I, Larson BL, Vuoristo JT et al. Comparison of effect of BMP-2, -4 , and -6 on in vitro cartilage formation of human adult stem cells from bone marrow stroma. Cell Tissue Res. 2005;320:269-76.

26. Eslaminejad MB, Nadri S. Murine mesenchymal stem cell isolated and expanded in low and high density culture system: surface antigen expression and osteogenic culture mineralization. In Vitro Cell Dev.Biol.Anim 2009;45:451-9.

27. Lee SY, Lim J, Khang $G$ et al. Enhanced ex vivo expansion of human adipose tissue-derived mesenchymal stromal cells by fibroblast growth factor-2 and dexamethasone. Tissue Eng Part A 2009;15:2491-9.

28. Javazon EH, Beggs KJ, Flake AW. Mesenchymal stem cells: paradoxes of passaging. Exp. Hematol. 2004;32:414-25.

29. Vacanti V, Kong E, Suzuki G et al. Phenotypic changes of adult porcine mesenchymal stem cells induced by prolonged passaging in culture. J.Cell Physiol 2005;205:194-201.

30. Bonab MM, Alimoghaddam K, Talebian F et al. Aging of mesenchymal stem cell in vitro. BMC.Cell Biol. 2006;7:14.

31. Liu L, DiGirolamo CM, Navarro PA et al. Telomerase deficiency impairs differentiation of mesenchymal stem cells. Exp.Cell Res. 2004;294:1-8.

32. Minguell JJ, Erices A, Conget P. Mesenchymal stem cells. Exp.Biol.Med.(Maywood.) 2001;226:507-20.

33. He F, Chen X, Pei M. Reconstruction of an in vitro tissue-specific microenvironment to rejuvenate synovium-derived stem cells for cartilage tissue engineering. Tissue Eng Part A 2009;15:3809-21.

34. Kihara T, Hirose M, Oshima A et al. Exogenous type I collagen facilitates osteogenic differentiation and acts as a substrate for mineralization of rat marrow mesenchymal stem cells in vitro. Biochem.Biophys. Res.Commun. 2006;341:1029-35.

35. Wakitani S, Goto T, Young RG et al. Repair of large full-thickness articular cartilage defects with allograft articular chondrocytes embedded in a collagen gel. Tissue Eng 1998;4:429-44.

36. Martel-Pelletier J, Boileau C, Pelletier JP et al. Cartilage in normal and osteoarthritis 
conditions. Best.Pract.Res.Clin.Rheumatol. 2008;22:351-84.

37. Ochi $\mathrm{M}$, Uchio $\mathrm{Y}, \mathrm{Kawasaki} \mathrm{K}$ et al. Transplantation of cartilage-like tissue made by tissue engineering in the treatment of cartilage defects of the knee. J.Bone Joint Surg.Br. 2002;84:571-8.

38. Lee CR, Grodzinsky AJ, Hsu HP et al. Effects of a cultured autologous chondrocyte-seeded type II collagen scaffold on the healing of a chondral defect in a canine model. J.Orthop. Res. 2003;21:272-81.

39. Pei $M, H e F$, Wei $L$ et al. Melatonin enhances cartilage matrix synthesis by porcine articular chondrocytes. J.Pineal Res. 2009;46:181-7.

40. Chen XD, Dusevich V, Feng JQ et al. Extracellular matrix made by bone marrow cells facilitates expansion of marrow-derived mesenchymal progenitor cells and prevents their differentiation into osteoblasts. J.Bone Miner.Res. 2007;22:1943-56.

41. Cukierman E, Pankov R, Stevens DR et al. Taking cell-matrix adhesions to the third dimension. Science 2001;294:1708-12.

42. Castello-Cros R, Cukierman E. Stromagenesis during tumorigenesis: characterization of tumor-associated fibroblasts and stroma-derived 3D matrices. Methods Mol.Biol. $2009 ; 522: 275-305$.

43. Castello-Cros R, Khan DR, Simons J et al. Staged stromal extracellular 3D matrices differentially regulate breast cancer cell responses through PI3K and beta1-integrins. BMC.Cancer 2009;9:94.

44. Lai Y, Sun Y, Skinner CM et al. Reconstitution of Marrow-derived Extracellular Matrix Ex Vivo: a Robust Culture System for Expanding Large-scale Highly Functional Human Mesenchymal Stem Cells. Stem Cells Dev. 2009.

45. Cukierman E, Pankov R, Yamada KM. Cell interactions with three-dimensional matrices. Curr.Opin. Cell Biol. 2002;14:633-9.

46. Bosnakovski D, Mizuno M, Kim G et al. Chondrogenic differentiation of bovine bone marrow mesenchymal stem cells (MSCs) in different hydrogels: influence of collagen type II extracellular matrix on MSC chondrogenesis. Biotechnol.Bioeng. 2006;93:1152-63.

47. Buma P, Pieper JS, van TT et al. Cross-linked type I and type II collagenous matrices 
for the repair of full-thickness articular cartilage defects--a study in rabbits. Biomaterials 2003;24:3255-63.

48. Vickers SM, Gotterbarm T, Spector M. Cross-linking affects cellular condensation and chondrogenesis in type II collagen-GAG scaffolds seeded with bone marrow-derived mesenchymal stem cells. J.Orthop.Res. 2010;28:1184-92.

49. Lee CR, Breinan HA, Nehrer S et al. Articular cartilage chondrocytes in type I and type II collagen-GAG matrices exhibit contractile behavior in vitro. Tissue Eng 2000;6:555-65.

50. Winer JP, Janmey PA, McCormick ME et al. Bone marrow-derived human mesenchymal stem cells become quiescent on soft substrates but remain responsive to chemical or mechanical stimuli. Tissue Eng Part A 2009;15:147-54.

51. Schlaepfer DD, Hanks SK, Hunter T et al. Integrin-mediated signal transduction linked to Ras pathway by GRB2 binding to focal adhesion kinase. Nature 1994;372:786-91.

52. Zhao JH, Reiske H, Guan JL. Regulation of the cell cycle by focal adhesion kinase. J.Cell Biol. 1998;143:1997-2008.

53. Zhu X, Assoian RK. Integrin-dependent activation of MAP kinase: a link to shape-dependent cell proliferation. Mol.Biol.Cell 1995;6:273-82.

54. Eliceiri BP, Puente XS, Hood JD et al. Src-mediated coupling of focal adhesion kinase to integrin alpha(v)beta5 in vascular endothelial growth factor signaling. J.Cell Biol. 2002;157:149-60.

55. Holtkotter $\mathrm{O}$, Nieswandt $\mathrm{B}$, Smyth $\mathrm{N}$ et al. Integrin alpha 2-deficient mice develop normally, are fertile, but display partially defective platelet interaction with collagen. J.Biol.Chem. 2002;277:10789-94.

56. Inoue O, Suzuki-Inoue K, Dean WL et al. Integrin alpha2beta1 mediates outside-in regulation of platelet spreading on collagen through activation of Src kinases and PLCgamma2. J. Cell Biol. 2003;160:769-80.

57. Sawhney RS, Cookson MM, Omar $Y$ et al. Integrin alpha2-mediated ERK and calpain activation play a critical role in cell adhesion and motility via focal adhesion kinase signaling: identification of a novel signaling pathway. J.Biol.Chem. 2006;281:8497-510. 
58. Lu Z, Doulabi BZ, Huang C et al. Collagen type II enhances chondrogenesis in adipose tissue-derived stem cells by affecting cell shape. Tissue Eng Part A 2010;16:81-90.

59. Even-Ram S, Yamada KM. Cell migration in 3D matrix. Curr.Opin.Cell Biol. 2005;17:524-32.

60. Green JA, Yamada KM. Three-dimensional microenvironments modulate fibroblast signaling responses. Adv.Drug Deliv. Rev. 2007;59:1293-8.

61. Pankov R, Endo $\mathrm{Y}$, Even-Ram $\mathrm{S}$ et al. A Rac switch regulates random versus directionally persistent cell migration. J. Cell Biol. 2005;170:793-802.

62. Rubio D, Garcia-Castro J, Martin MC et al. Spontaneous human adult stem cell transformation. Cancer Res. 2005;65:3035-9.

63. Deng J, Petersen BE, Steindler DA et al. Mesenchymal stem cells spontaneously express neural proteins in culture and are neurogenic after transplantation. Stem Cells 2006;24:1054-64.

64. Kinsella MG, Bressler SL, Wight TN. The regulated synthesis of versican, decorin, and biglycan: extracellular matrix proteoglycans that influence cellular phenotype. Crit Rev.Eukaryot.Gene Expr. 2004;14:203-34.

65. Kresse H, Schonherr E. Proteoglycans of the extracellular matrix and growth control. J.Cell Physiol 2001;189:266-74.

66. Zhang YE. Non-Smad pathways in TGF-beta signaling. Cell Res. 2009;19:128-39.

67. Watanabe H, de Caestecker MP, Yamada Y. Transcriptional cross-talk between Smad, ERK1/2, and p38 mitogen-activated protein kinase pathways regulates transforming growth factor-beta-induced aggrecan gene expression in chondrogenic ATDC5 cells. J.Biol.Chem. 2001;276:14466-73.

68. Bobick BE, Kulyk WM. The MEK-ERK signaling pathway is a negative regulator of cartilage-specific gene expression in embryonic limb mesenchyme. J.Biol.Chem. 2004;279:4588-95.

69. Kim HJ, Im GI. The effects of ERK1/2 inhibitor on the chondrogenesis of bone marrow- and adipose tissue-derived multipotent mesenchymal stromal cells. Tissue Eng Part A 2010;16:851-60. 
70. Li J, Zhao Z, Liu J et al. MEK/ERK and p38 MAPK regulate chondrogenesis of rat bone marrow mesenchymal stem cells through delicate interaction with TGF-beta1/Smads pathway. Cell Prolif. 2010;43:333-43.

71. Miedlich SU, Zalutskaya A, Zhu ED et al. Phosphate-induced apoptosis of hypertrophic chondrocytes is associated with a decrease in mitochondrial membrane potential and is dependent upon Erk1/2 phosphorylation. J.Biol.Chem. 2010;285:18270-5.

72. Pelttari K, Winter A, Steck E et al. Premature induction of hypertrophy during in vitro chondrogenesis of human mesenchymal stem cells correlates with calcification and vascular invasion after ectopic transplantation in SCID mice. Arthritis Rheum. 2006;54:3254-66.

73. Pelttari K, Steck E, Richter W. The use of mesenchymal stem cells for chondrogenesis. Injury 2008;39 Suppl 1:S58-S65. 


\section{FIGURE LEGENDS}

Figure 7.1 (A) Histology and immunohistochemistry of SDSC-derived ECM (S-ECM) and chondrocyte-derived ECM (C-ECM). Alcian blue and alcian blue staining was used to detect sulfated glycosaminoglycans (GAGs), and immunohistochemistry staining (IHC) was for type I (COL I) and type II (COL II) collagen; (B) S-ECM and C-ECM induces a cell morphology change in SDSCs and chondrocytes in monolayer expansion; (C) the mean cell number of SDSCs harvested from passage 4 to passage 6 on the three different substrates (Plastic, S-ECM and C-ECM); (D) the mean cell number of chondrocytes harvested on Plastic, S-ECM and C-ECM. Data are shown as average \pm SD for $n=4$.

Figure 7.2 Histology and immunohistochemistry of SDSC pellets of Plastic, S-ECM and C-ECM groups induced chondrogenic differentiation. Alcian blue (blue) staining was used to detect sulfated glycosaminoglycans (GAGs), and immunohistochemistry was for type I (COL I), II (COL II) and X collagens (COL X).

Figure 7.3 (A) Biochemistry analysis for GAGs of SDSC pellets were measured at day 0, day 7 and day 14. (B), DNA content of SDSC pellets changes during 14 days normalized by the content of pellets at day $0 .(C)$, chondrogenic index (the ratio of GAGs and DNA) showed the degree of chondrogenic differentiation of SDSCs. (D) to (G) Real-time PCR to examine the chondrogenic marker gene expression level (type I and II collagen, aggrecan, sox-9) normalized by 18S rRNA. $(\mathrm{H})$ and $(\mathrm{I})$ the hypertrophic gene expression (type $\mathrm{X}$ collagen and MMP13) was tested in the SDSC pellets. Data are shown as average \pm SD for $n=4$.

Figure 7.4 Immunoblotting of the SDSC chondrogenic pellet was used to detect phospho-TGF $\beta$ RII (Tyr 424), Sox9 and type II collagen expression level. $\beta$ actin was used as an internal control for protein loading.

Figure 7.5 (A) Histology and immunohistochemistry of chondrocyte pellets of Plastic, S-ECM and C-ECM groups that underwent induced chondrogenic differentiation. Alcian blue (blue) 
staining was used to detect GAGs, and immunohistochemistry determined type I (COL I), II (COL II) and X collagen (COL X).

Figure 7.6 (A) Biochemistry analysis for GAGs of SDSC pellets were measured at day 0, day 7 and day 14. (B), DNA content of chondrocyte pellets changes during 14 days normalized by the content of pellets at day $0 .(C)$, chondrogenic index (the ratio of GAGs and DNA) showed the degree of chondrogenic differentiation of chondrocytes. (D) to (G) Real-time PCR to exam the chondrogenic marker gene expression level (type I and II collagen, aggrecan, sox-9) normalized by $18 \mathrm{~S}$ rRNA. $(\mathrm{H})$ and $(\mathrm{I})$ the hypertrophic gene expression (type $\mathrm{X}$ collagen and MMP13) was tested in the chondrocyte pellets. Data are shown as average \pm SD for $n=4$.

Figure 7.7 Immunoblotting of chondrocyte chondrogenic pellet was used to detect phospho-TGF $\beta$ RII (Tyr 424), Sox9 and type II collagen expression level. $\beta$ actin was used as an internal control for protein loading. 
Figure 7.1

A
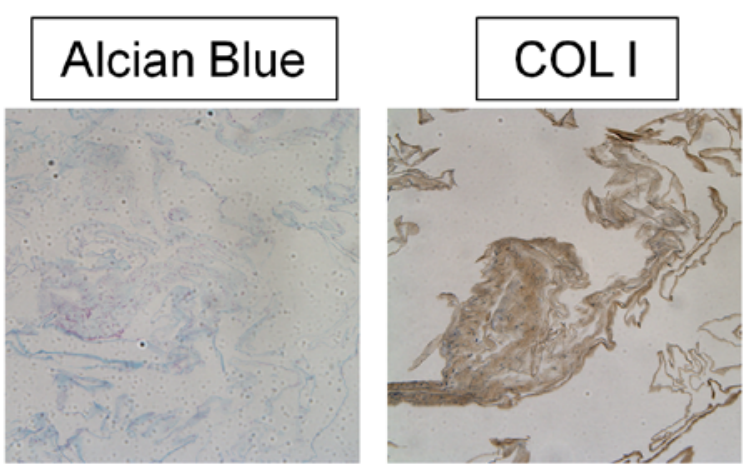

COL II

S-ECM

C-ECM
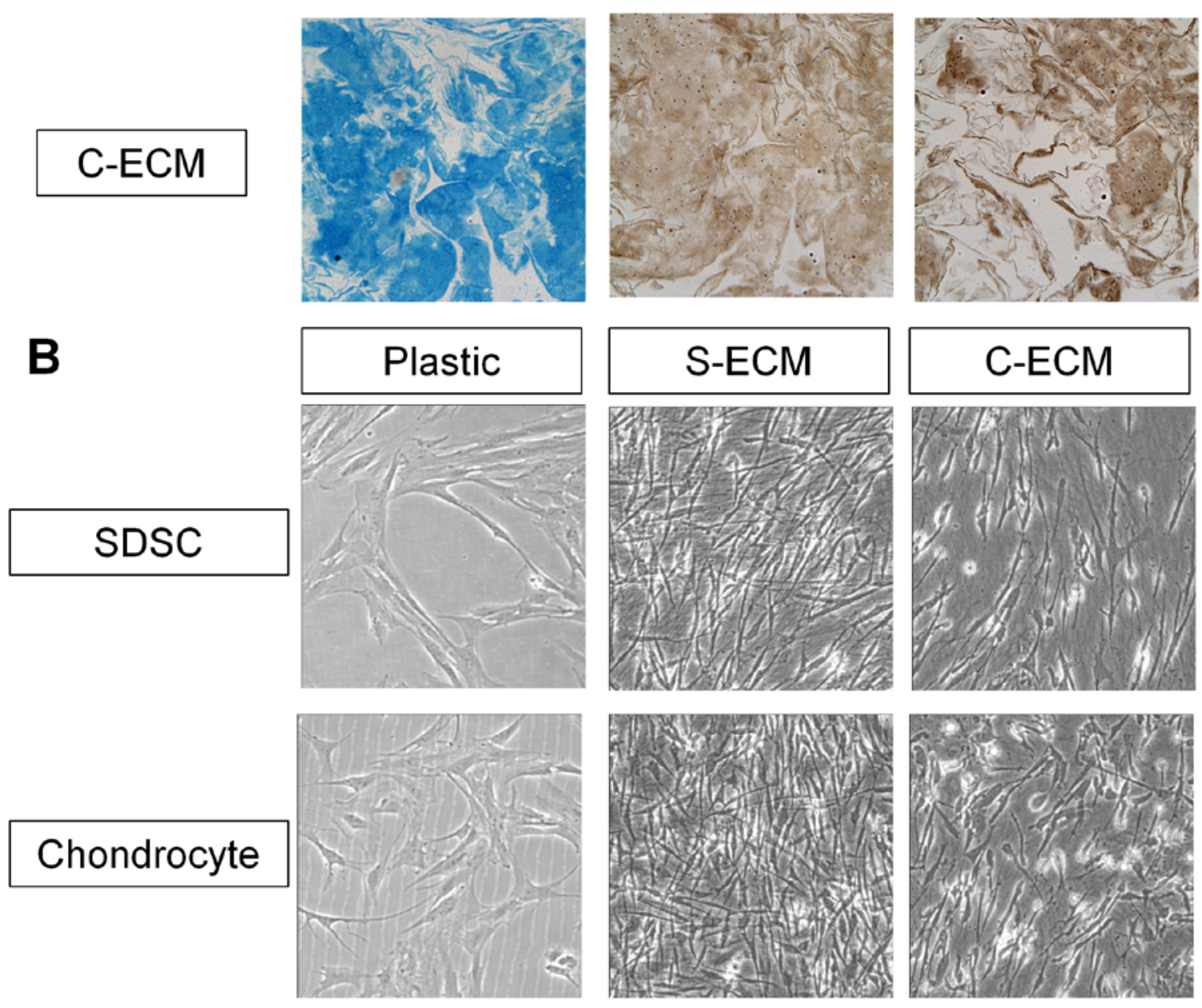

C
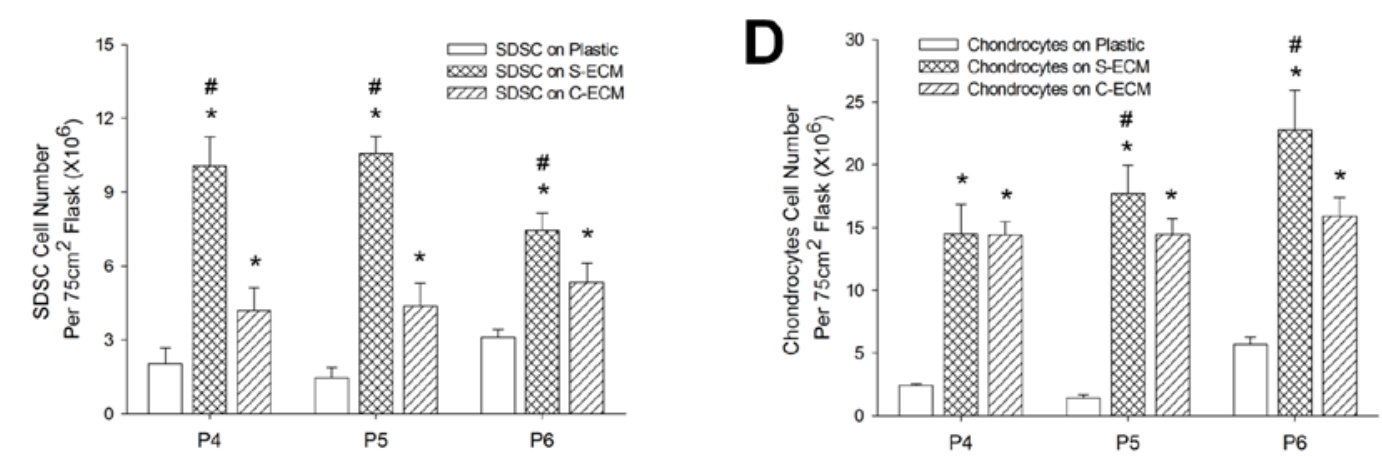
Figure 7.2

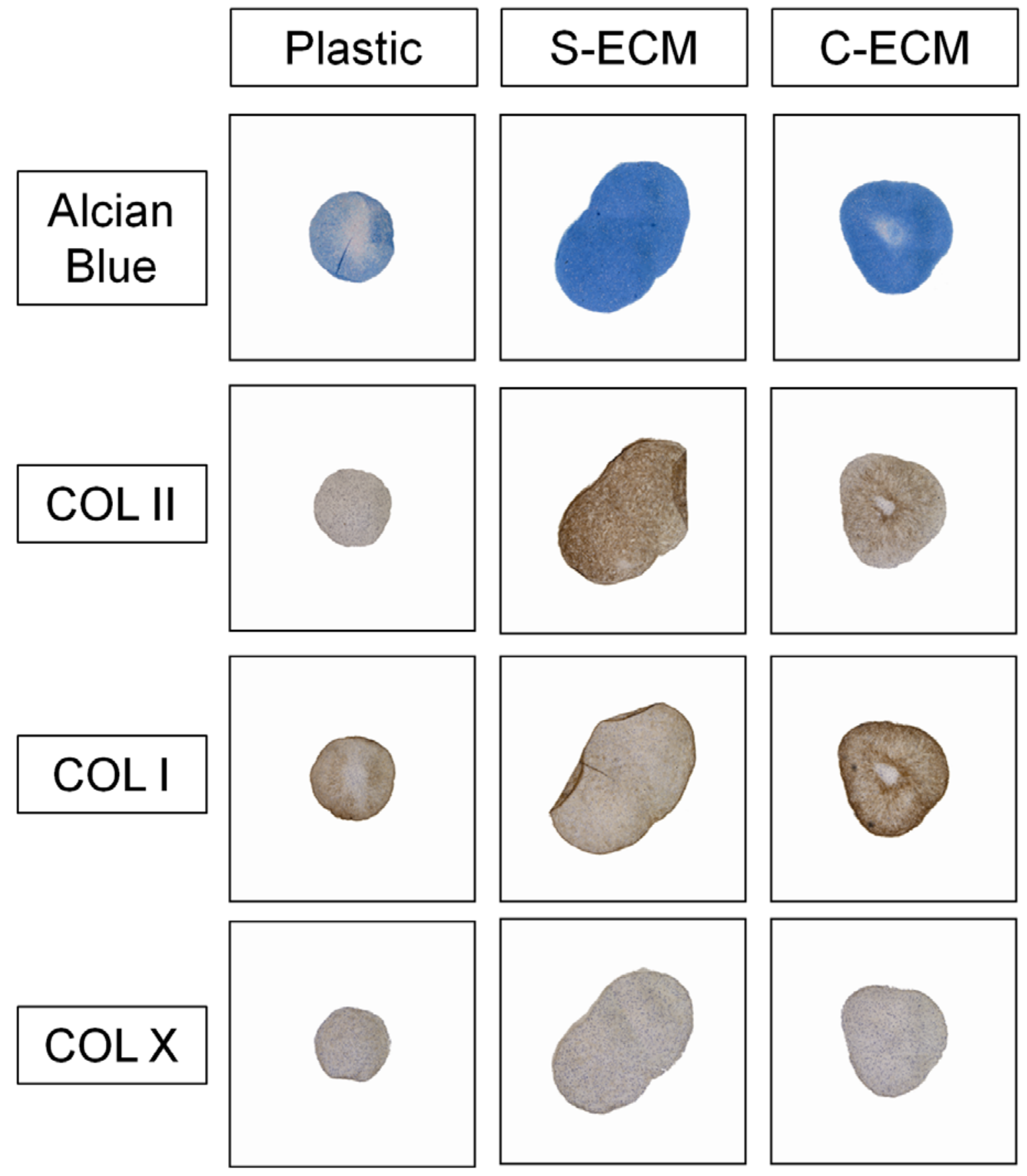




\section{Figure 7.3}

A

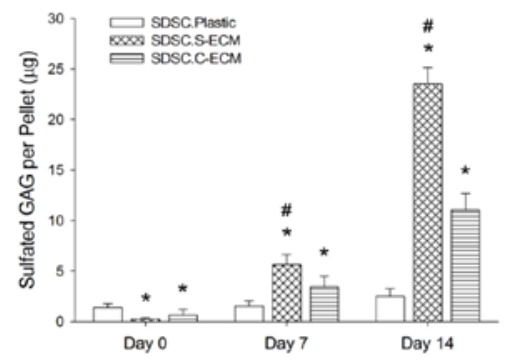

D

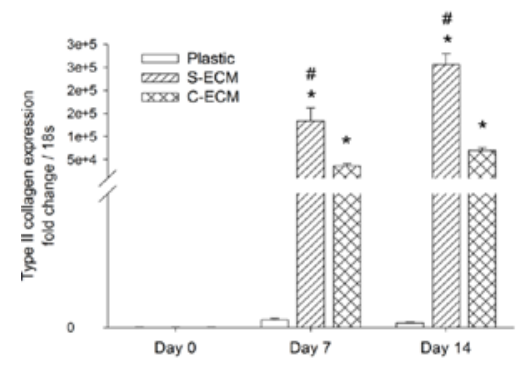

G

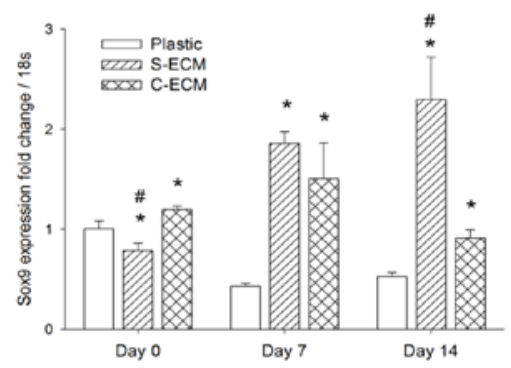

B

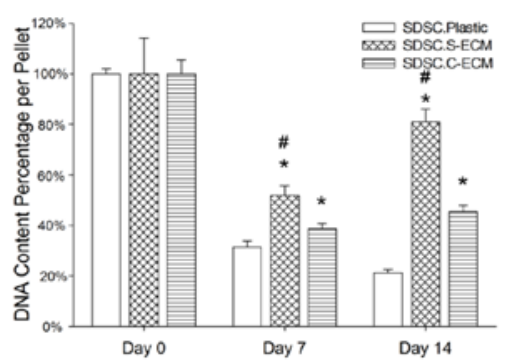

E

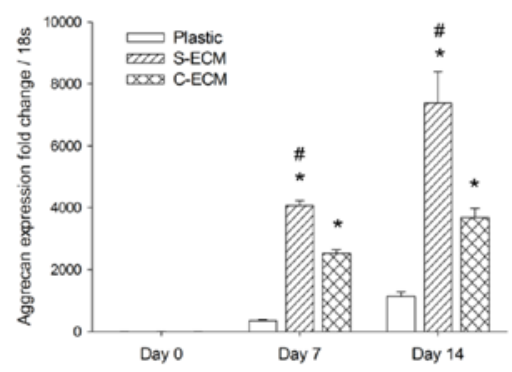

H

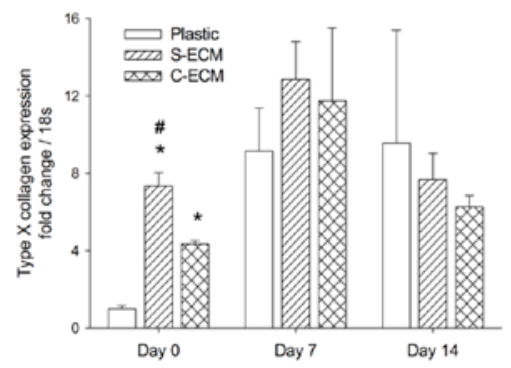

C

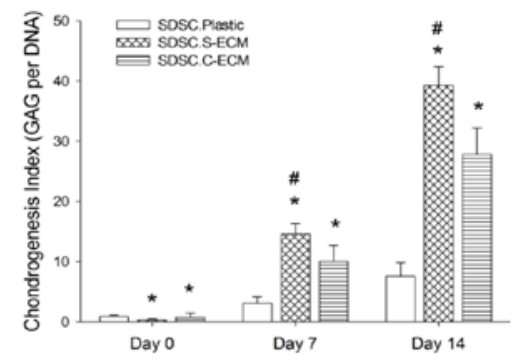

F

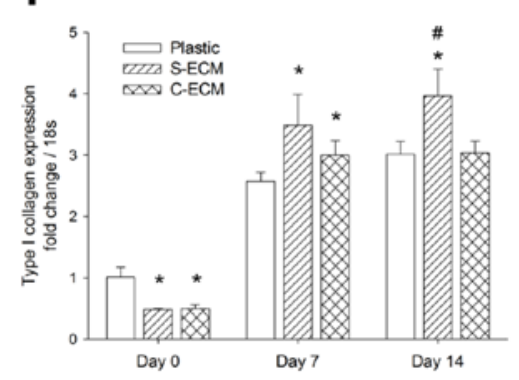

I

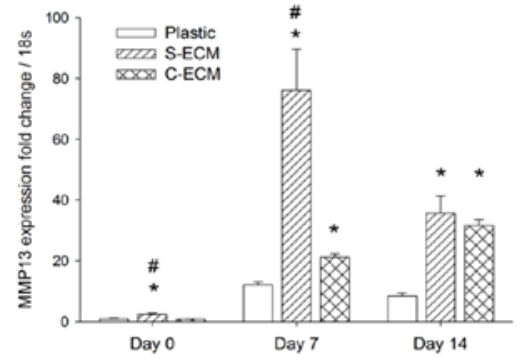


Figure 7.4

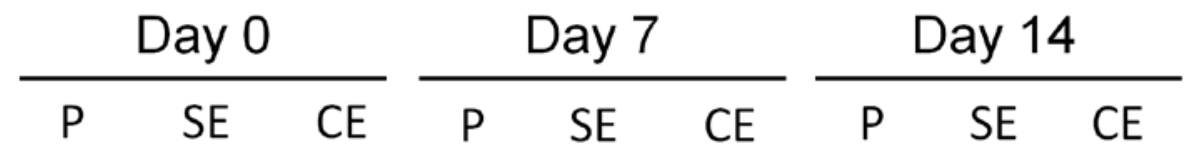

$p$-TGF- $\beta R$ R II

Sox 9

Type II Collagen

$p-E R K$
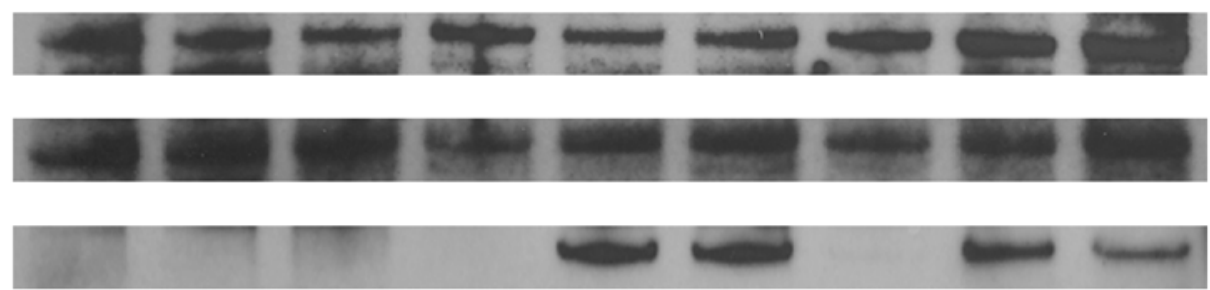

ERK

$\beta$ actin 
Figure 7.5

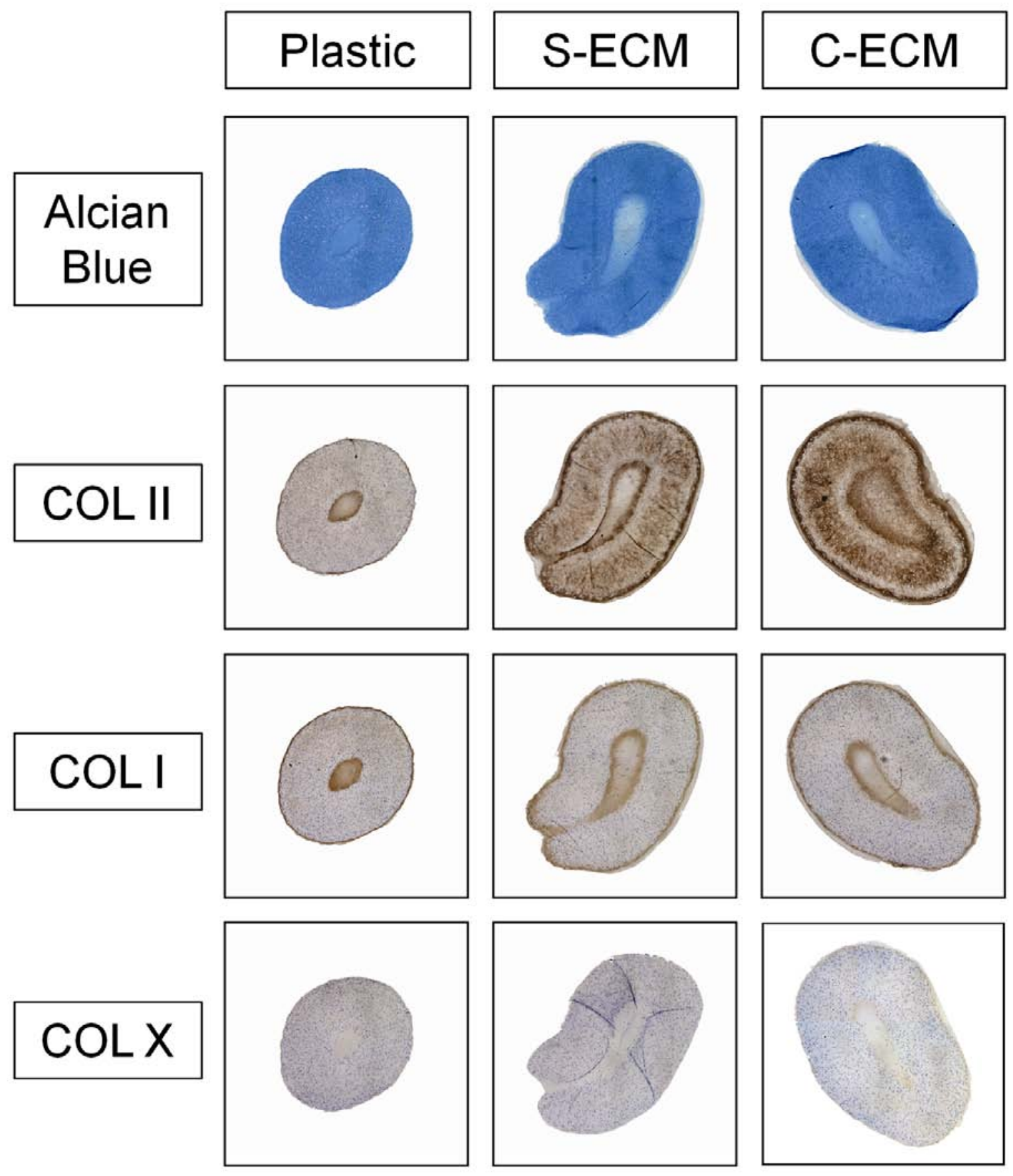


Figure 7.6

A

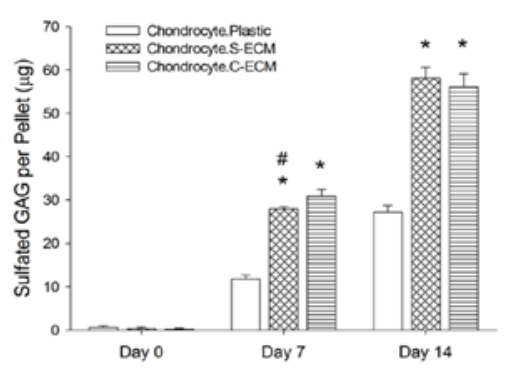

D

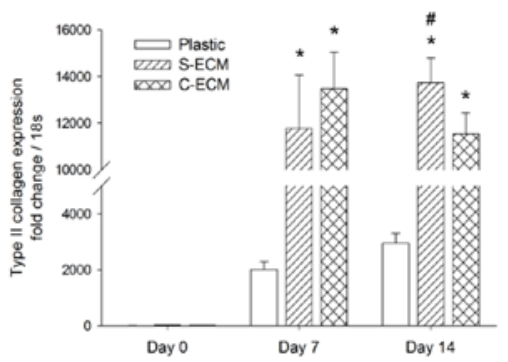

G

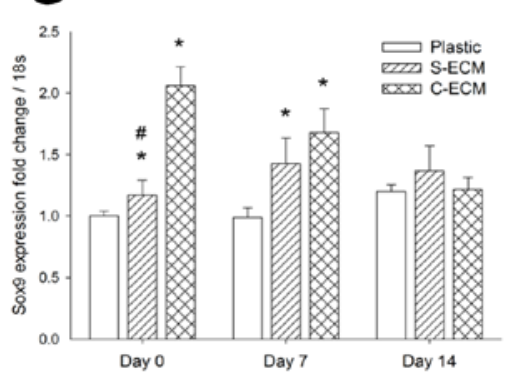

B

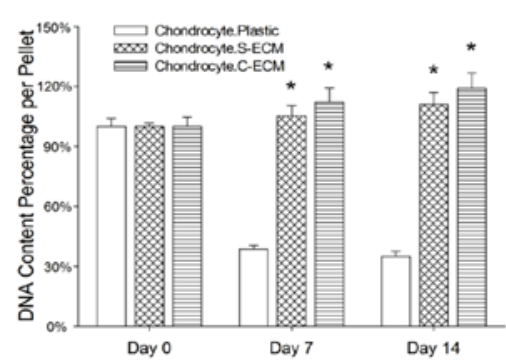

E

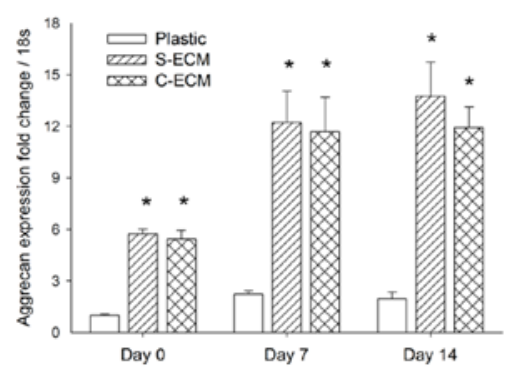

H

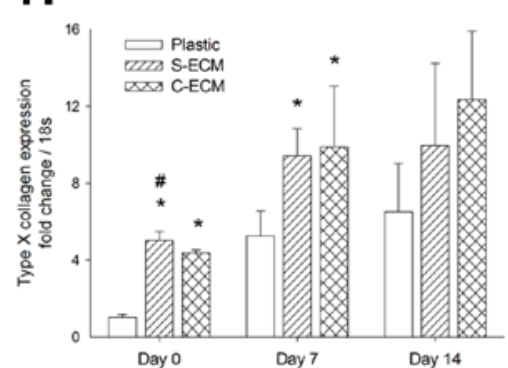

C

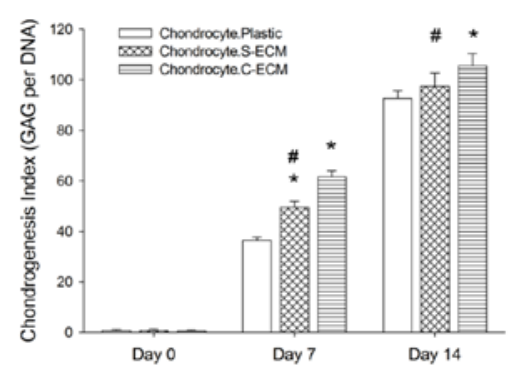

F
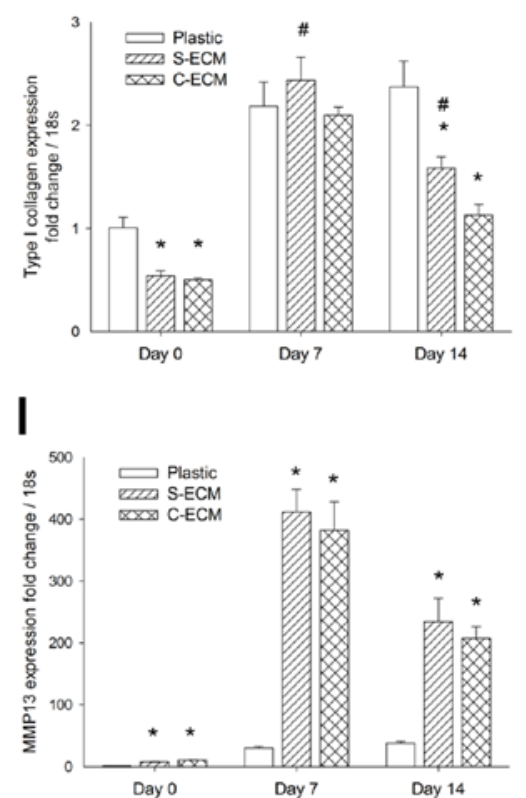
Figure 7.7

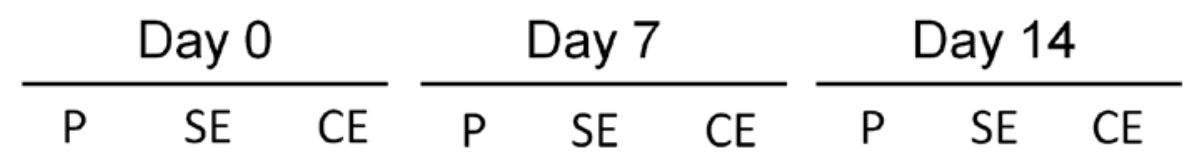

p-TGF- $\beta$ R II

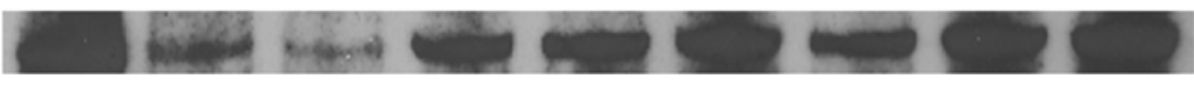

Sox 9

Type II Collagen

$$
p \text { - ERK }
$$

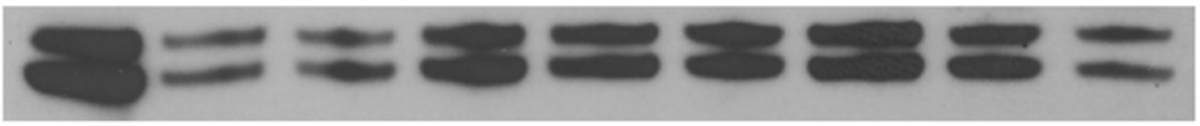

\section{ERK}

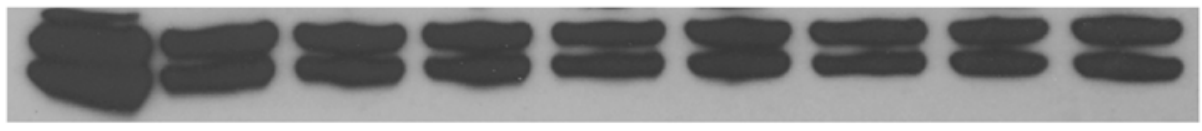

$\beta$ actin 


\section{Chapter 8:}

\section{General Discussion}


The objective of this dissertation is to develop a novel strategy for cell-based cartilage regeneration (including mesenchymal stem cells and autologous differentiated cells) that can promote cell expandability, enhance chondrogenic differentiation, and improve the repair effect for cartilage defects in animal studies and clinical trials. A native cell-deposited three-dimensional (3D) extracellular matrix (ECM) provides a promising approach to improve current in vitro MSC expansion system not only increasing cell proliferative ability but also enhancing chondrogenic differentiation potential. The central hypothesis of this dissertation is that the natural three-dimensional cell-deposited extracellular matrix can provide a proper microenvironment to improve MSC self-renewal and multipotent differentiation. This natural 3D ECM can also improve the current procedures of autologous cell transplantation for cartilage defects and intervertebral disc degeneration.

\subsection{Extracellular matrix improves MSC self-renewal}

Mesenchymal stem cells are attractive for tissue repair and regeneration, because of their multipotential differentiation to various lineages, including chondrocytes for cartilage repair, ${ }^{1 ; 2}$ osteoblasts for bone reconstitution, ${ }^{3}$ adipocytes for adipose tissue regeneration, ${ }^{4}$ cardiac myoblasts for cardiovascular repair, ${ }^{5 ; 6}$ myoblasts for skeletal muscle regeneration ${ }^{7}$ and neuronal cell for neurological system repair. ${ }^{8}$ It is extremely important for MSC to obtain sufficient cell numbers and to maintain their stemness in the in vitro culture process. ${ }^{9}$ Sufficiency of MSCs is a basic requirement for MSC-based tissue regeneration. Typically the intracellular injection therapy to repair partial-thickness cartilage defects requires minimal 10 million cells for a single injection. ${ }^{10}$ For infracted heart repair 4 million cells are necessary for a single injection. ${ }^{11}$ Moreover, in cartilage repair the loss of regenerative cells will occur due to leakage or cell apoptosis. ${ }^{12}$ In autologous chondrocyte transplantation, the leakage of injected chondrocytes from repaired sites usually results in the failure of implantation, multiple complications and extra cost of patients. In clinical practice, cartilage repair using autologous chondrocyte transplantation usually requires several injections instead of a single injection to

enhance the repair effect. ${ }^{12-14}$ Besides, apoptosis or cell death are also induced by inflammatory cytokines, metalloproteinase, intracellular oxygen species or mechanical 
forces. ${ }^{9}$ Osteoarthritis, which was considered a degenerative disease, now is more realized as an immune disorder. It is evident that several inflammatory factors, including interleukin I $\beta$ (IL-I $\beta$ ), IL-I7, IL-I8, TNF- $\alpha$ and oncostatin M (OSM), are responsible mediators for cartilage degeneration and osteoarthritis. These inflammatory factors not only stimulate the turnover of cartilage extracellular matrix but also increase chondrocyte apoptosis. ${ }^{15}$ Overall, to obtain adequate cell number is one of the most important issues in cell-based tissue regeneration.

The current in vitro culture condition limits the use of MSC-based tissue regeneration. Once isolated from the in vivo microenvironment, MSCs rapidly lost the ability of proliferation and multipotent differentiation like aged cells. ${ }^{16-18}$ In this dissertation, we tried to use a native 3D cell-deposited ECM to replace conventional MSC expansion process, not only shortening expansion time but also increasing yielded cell numbers. Starting at a relatively high seeding density $\left(3,000\right.$ cells $\left./ \mathrm{cm}^{2}\right)$, it only took 5 - 6 days to reach $90 \%$ confluence, while at low seeding density $\left(1,000\right.$ cells $\left./ \mathrm{cm}^{2}\right)$ the expansion period extented to 7 to 10 days depending on specific cell types. More importantly, the complete confluence did not inhibit cell proliferation, since cells continued to grow overlaply in 3D format. ${ }^{19 ; 20}$ The adequate cell number produced by ECM expansion system provided sufficient cells for transplantation.

The high proliferative rate on ECM is related to activated cyclin D1 and reduced intracellular reactive oxygen species (Chapter 3 and Chapter 6). Cyclin D1 is proven to control cell cycle progression through $\mathrm{G} 1$ phase into S phase. The steady-state level of phosphorylated ERK1/2 is essential for cyclin D1 phosphorylation and translocation to promote cell cycle from G1 to S phase, while inhibition of ERK1/2 or upstream kinases such as MAPK results in decreased activity of cyclin D1. ${ }^{21}$ Except MAPK cascade, Rac1 is another distinct mediator to activate cyclin D1 and promote cell-cycle. ${ }^{22 ; 23}$ Page et al. showed that overexpression of activated Rac increased the activity of cyclin D1, but the transcription of mutant Rac attenuated cyclin D1 activity. The Rac1 signaling pathway is completely independent of ERK activation. Westwick et al. showed that Rac1 regulated the activity of at least four protein kinases including PAK, JNK, p38 and serum response factor (SRF), among which Rac1 stimulated the transcription of cyclin D1 promoter associated with PAK binding. ${ }^{22}$ Besides, they also showed that low level of intracellular ROS induced 
activation of cyclin D1 and antioxidant treatment attenuated Rac1-induced cyclin D1 promotor activity. ${ }^{24}$ In this dissertation, human BMSCs expanded on ECM showed a sustained activation of ERK1/2 and cyclin D1. Besides, phosphatidylinositol 3-kinase (PI3-kinase) signaling pathway also modulates the activity of cyclin D1 and the progression from $\mathrm{G} 1$ to $\mathrm{S}$ phase of cell cycle. Jirmanova et al. showed the evidence that activated PI3-kinase induced an increased steady-state level of cyclin D1 and the decrease of GSK3 $\beta$-dependent rate of cyclin D1 degradation. ${ }^{25}$ Considering the regulatory effect of PI3-kinase/Akt signaling pathway in TGF- $\beta$ induced chondrogenesis, it is possible that PI3-kinase influences cell proliferation and chondrogenic potential when expanding on ECM; this particular signaling pathway will be examined in the future studies. One of the limitations in this dissertation is that the assay to study the effect of inhibition of Src or ERK1/2 on cell proliferation is not available due to serious apoptotic response in a short time.

Intracellular reactive oxygen species (ROS) are proven to play an important role in cell adhesion, migration, proliferation and cell survival. ${ }^{26-29}$ The low concentration of ROS had a promotional effect on cell adhesion and the threshold, demonstrated by Lamari et al., was between 5 to 7 nanmoles / $\mathrm{mL}$ of superoxide anions, whereas the high level of ROS resulted in cell death. ${ }^{30}$ High level of ROS had an inhibitory influence on cell proliferation by arresting cell cycle at G1, S, and G2 phases through down-regulation of cyclin D1 and D3. ${ }^{31}$ The high level of ROS or treatment of hydrogen peroxide resulted in cyclin D1 degradation, which was reversed in the presence of antioxidants or overexpression of hypoxia-inducible factor (HIF) $1 \alpha^{32} \mathrm{Kim}$ et al. also showed that the increase of intracellular ROS generation arrested cell cycle at $G_{0}-G_{1}$ phase by inhibition of cyclin D1/CDK4 complex. ${ }^{33}$ Besides, Lai et al. reported an elevated activity of telomerase in human BMSCs expanded on a similar natural 3D ECM that might also promote cell proliferation. ${ }^{20}$

\subsection{D cell-deposited ECM is different from 2D reconstructed ECM}

Traditional 2D reconstructed ECM uses monomeric proteins such as fibronectin and laminin-1 to coat substrates and showed little effect on cell morphology or proliferation. ${ }^{34}$ Tsai 
et al. provided evidence that type I collagen achieved better result in promoting human MSC proliferation and osteogenesis than fibronectin, laminins or gelatin. The improved osteogenic differentiation was related to activated ERK and Akt pathways instead of FAK; the inhibition of ERK attenuated osteogenic process. ${ }^{35}$ More importantly, the 3D environment can result in different cell responses and behaviors from the 2D ones. Fassett et al. showed that hepatocytes in type I collagen gel remained round and arrested growth, whereas on the monomeric type I collagen coating film the cells spreaded and proliferated. The different activity of cAMP-dependent protein kinase (PKA) was responsible for the distinct cell proliferation responses, because inhibition of PAK restored ERK activation and cyclin D1 to promote cell cycle from $\mathrm{G} 1$ to $\mathrm{S}$ phase. ${ }^{36}$ However, 2D ECM containing multiple proteins was able to induce stem cell self-renewal and to promote chondrogenic differentiation. ${ }^{34} 3 \mathrm{D}$ culture system has been proven more efficient in cell expansion and preserving multipotent characters of MSCs in vitro. Majd et al. recently developed a new 3D dynamic culture for MSCs that yielded 10-fold cells more than conventional plastic culture in nine weeks; it also maintained MSC multipotential to differentiate into osteoblasts, adipocytes, chondrocytes and myoblasts that was lost in the conventional culture. ${ }^{37}$

The compositions of MSC-deposited ECM included type I collagen, fibronectin, laminins and decorin detected by immunohistochemistry..$^{19 ; 20 ; 38 ; 39}$ Proteomics provides a novel method to analyze the components in ECM. A detailed analysis of the compositions in ECM can help us understand the interactions between cells and ECM. Overall, the improved proliferative and chondrogenic potential of MSCs induced by ECM is related to the integration of multiple matrix molecules and the unique three-dimensional structure.

MSCs on 3D ECM exhibited distinct behaviors compared to conventional plastic flasks or constructed 2D ECM. Cells migrated along the fibrils of ECM instead of random movement on plastic flasks. The directional migration was closely related to decreased activity of Rac1 that was demonstrated to control cell migration. ${ }^{40}$ Moreover, the uneven distribution of matrix components resulted in different rigidity of ECM. Cells liked to move from an area with higher flexibility to a region with more rigidity. ${ }^{41}$ On conventional plastic flasks or reconstructed 2D 
ECM the well-distribution of molecules leaded to random cell migration. Also the elasticity of ECM affected cell shape and proliferation. For example, fibroblasts appeared round shape on soft substrates but changed to stressed shape when they were cultured on matrix with high rigidity. Winer et al. compared the effect of substrates with different rigidity on human BMSCs and demonstrated that cells became quiescent on soft matrix (250 Pa) but resumed proliferation when transferring to a stiff substrate. ${ }^{42}$

During cell adhesion, 3D ECM induced decreased FAK related to focal adhesion and formed new contacts with cells. The MAPK cascade and ERK1/2 in cell adhesion, migration and proliferation has been studied in depth. Sustained activation of ERK1/2 was necessary for cell cycle progession by induction of cyclin D1; the inhibition of ERK1/2 resulted in reduction of cyclin D1 and arrest at $G_{1}$ phase. ${ }^{43}$ The attachment of cells onto ECM, including fibronectin, vitronectin, laminins and collagens, induced the activation of integrin $\alpha_{1}, \alpha_{2}, \alpha_{5}, \alpha_{v}, \beta_{1}, \beta_{3}$ and $\beta_{5}$. Integrin system transduced extracellular signals into intracellular pathways by activating FAK, Src, MAPK cascade, ERK1/2 and cyclin proteins to control cell cycles. ${ }^{44 ; 45}$ Park et al. cultured embryonic stem cells on fibronectin-coated surfaces and showed an upregulation of integrin $\beta_{1}, F A K$, Src, ERK1/2, Akt and caveolin-1. The intracellular signaling pathways induced by fibronectin increased cell cycle regulatory proteins such as cyclin D1 and cyclin E. The inhibition of integrin $\beta_{1}$ or blockage of kinases such as Src, FAK, caveolin-1, Akt or ERK1/2 resulted in significantly decreased $\left[{ }^{3} \mathrm{H}\right]$-thymidine incorporation and attenuated cell cycle regulatory proteins. ${ }^{46}$ Roovers et al. showed that integrin $\alpha_{5} \beta_{1}$ controlled cyclin D1 expression through sustained activation of ERK1/2 on fibronectin to promote cell cycle. ${ }^{47}$ Cukierman et al. used a similar 3D ECM deposited by fibroblasts to study cell adhesion and demonstrated that integrin $\alpha_{5} \beta_{1}$ translocated to the region of fibronectin and induced intracellular signaling transduction. ${ }^{39}$ On fibronectin-coated surface, integrin $\alpha_{5} \beta_{1}$ mediated epithelial cell proliferation through epithelial growth factor (EGF) receptor. Kuwada et al. showed that, in the presence of EGF receptor antagonistic antibody, epithelia cells overexpressed with integrin $\alpha_{5}$ grew at a normal rate, while control group was inhibited in cell proliferation. ${ }^{48}$ Meanwhile, integrin $\alpha_{v}$ activation in 3D collage gel contributed cell survival through phosphorylation of MAPK and ERK1/2, while the inhibition of ERK1/2 induced 
serious cell apoptosis. ${ }^{49}$

\subsection{The interaction between integrin system and 3D ECM}

In Chapter 6, integrin $\alpha_{2}$ and $\beta_{5}$, receptors for fibrillar collagens, showed an elevated level. The elevated level of integrin $\alpha_{2}$ was closely related to focal adhesion and cell motility. Sawhney et al. identified the role of integrin $\alpha_{2}$ in cell adhesion that activated FAK, ERK1/2 and $\mu$-calpain pathways on type IV collagen. Inhibition of integrin $\alpha_{2}$ by a specific antibody suppressed FAK activation and ERK1/2 phosphorylation. ${ }^{50}$ Integrin $\alpha_{2} \beta_{1}$ was proven to induce cyclin expression and promote cell cycle on type IV collagen. This process involved activation of the ERK1/2 signaling pathway and the production of ROS by NADPH oxidase. ${ }^{51}$ In this dissertation, increased integrin $\alpha_{2}$ may result in activation of ERK1/2. Meanwhile, integrin $\alpha_{2}$-regulated cell adhesion and migration were modulated through activation of Src and were completely inhibited in the presence of Src inhibitor PP2. ${ }^{52}$ Overall, Integrin $\alpha_{2}$ plays an important role in cell adhesion and migration involving FAK, Src, p38 and MAPK cascade signaling transduction and contributes to cell-cycle progression. Besides, Priestley et al. demonstrated the role of integrin $\alpha_{4}$ in stem cell self-renewal by knocking-out of integrin $\alpha_{4}$ that seriously restricted cell proliferation. ${ }^{53}$ In this dissertation, we found an increased level of integrin $\alpha_{4}$ in human BMSCs expanded on ECM that was possibly related to the high rate of cell self-renewal (Chapter 6).

Integrin $\beta_{5}$ plays an important role in cell migration and proliferation proven by previous studies. Zhang et al. showed that in human breast carcinoma cells p21-activated kinase 4 (PAK4) contributed to cell migration; PAK4 attached to integrin $\beta_{5}$ cytoplasmic domain. Cell migration involved the engagement of integrin $\alpha_{v} \beta_{5}$ and extracellular matrix such as vitronectin; it induced a redistribution of PAK from cytosol to dynamic lamellipodial structures. ${ }^{54}$ Braam et al. also provided evidence that vitronectin-coated surface promoted human embryonic stem cell attachment and self-renewal through integrin $\alpha_{\mathrm{v}} \beta_{5}{ }^{55}$ Cukierman and his colleagues used a similar ECM deposited by human fibroblast and showed that ECM induced activation of $\mathrm{PI} K \mathrm{~K}$ and $\mathrm{Akt} / \mathrm{PKB}$ through the integrin $\beta_{1}$ dependent signaling 
pathway. ${ }^{56}$ In this dissertation, we found other integrin receptors at a decreased or unchanged level. However, in future it is important to establish a full analysis of integrin and other ECM receptors, not only by flowcytometry but also using immunofluorescence.

Intracellular reactive oxygen species affect cell adhesion, survival and proliferation. The increase of ROS inhibited cell adhesion and migration by decreasing the activity of focal adhesion-related and integrin-related molecules. ${ }^{57}$ Song et al. provided evidence that hydrogen peroxide treatment induced detachment of MSCs and significantly decreased cell adhesion and migration. The cell detachment was caused by decreased integrin $\alpha_{\mathrm{v}} \beta_{1}$ and focal adhesion-related molecules such as FAK and Src in the presence of high level of intracellular ROS. ${ }^{57}$ Moreover, ROS-induced cell adhesion was related to the modification of integrin system by altering the exposure of integrin outside cell surface or their clustering. Low level of ROS decreased the expression of integrin $\alpha_{5}$ but increased $\alpha_{v}$ that was related to cell adhesion. ${ }^{30 ; 58-60}$ In this dissertation, we found human BMSCs expanded on ECM showed decrease of integrin $\alpha_{5}$ that was possibly induced by the low level of ROS. Meanwhile, integrin $\beta_{4}$ had a negatively regulatory effect on the production of ROS. Lv et al. showed that inhibition of integrin $\beta_{4}$ in primary mouse neuron cells elevated significant apoptosis and declined cell viability; the process was associated with increased activity of NADPH oxidase and production of intracellular ROS. ${ }^{61}$ In this dissertation we found that integrin $\beta_{4}$ slightly increased when human BMSCs were cultured on ECM that possibly was related to the declined level of ROS.

In this dissertation, flowcytometry showed that the expression of integrin $\alpha_{1}, \alpha_{3}, \alpha_{5}, \beta_{1}$ and $\beta_{3}$ decreased compared to those expanded on plastic culture (Chapter 6). Hayashi et al. cultured embryonic stem cells on ECM to promote cell self-renewal and showed that the expression of fibronectin- and laminin-binding integrin subunits increased while some collagen-binding integrins down-regulated. The overexpression of collagen-binding integrin subunits induced stem cell differentiation instead of self-renewal. ${ }^{62}$ Considering the down-regulated expression of certain integrins after expansion on ECM (Chapter 6), it was possible that inactivation of certain integrins was essential to promote stem cell self-renewal, 
and it will be studied in future.

The central role of Src in focal adhesion has been well studied. In FAK-dysfunctional cells, overexpression of activated Src can rescue cell apoptotic response. The inhibition of Src significantly increased adhesion-dependent apoptosis. ${ }^{63}$ Activated Src can enhance the expression of integrin $\alpha_{2}$ through protein kinase C- $\zeta$ in fibroblasts. ${ }^{63} \mathrm{Xu}$ et al. demonstrated that a three-dimensional collagen substrate increased integrin $\alpha_{2}$ and it was mediated by protein kinase $C-\zeta{ }^{64 ; 65}$ Moreover, Eliceiri et al. reported that Src was indispensible during the formation of $\mathrm{FAK} /$ integrin $\alpha_{\mathrm{v}} \beta_{5}$ complex, when endothelial cells attached to ECM or were activated by vascular endothelial growth factor (VEGF). The complex of FAK/ $\alpha_{v} \beta_{5}$ and the activity of Src were critical to VEGF-induced angiogenesis. ${ }^{66}$

In Chapter 6 human BMSCs cultured on ECM responded a higher level of apoptosis than those on conventional plastic flasks, suggested by an increased immunofluorescence intensity of annexin V. In endothelial cells and terminally differentiated chondrocytes, annexin $\mathrm{V}$ binded to integrin $\beta_{5}$ and activated protein kinase $\mathrm{C} \alpha(\mathrm{PKC} \alpha)$. Activated PKCa induced an increase of caspase-3 activity and leaded to ultimate apoptosis. ${ }^{67-69}$ Integrin $\beta_{5}$ showed an increase in BMSCs expanded on ECM that might correlate to the elevated level of annexin V, but the detail mechanism and the potential effects on MSCs are still unclear. One possible explanation is that in the human BMSCs there were mixture cell populations containing healthy and aged cells. The healthy BMSCs had a higher proliferative rate that acquired most of the nutrients, and had a faster migration to occupy most of the space on ECM. The insufficiency of nutrients and space induced apoptosis in aged cells. So the overall increase of proliferation was due to the self-renewal in healthy BMSCs and the increase of apoptosis was because of the cell death in aged BMSCs.

The robust proliferative rate of MSCs or differentiated cells expanded on ECM aroused another potential issue: were ECM-expanded cells uncontrollable or likely to form tumor? In this dissertation, cells expanded on ECM were reversible when culture environment changed. In Chapter 4, chondrocytes were first cultured on ECM for four passages and then transferred 
to conventional plastic culture for two passages; cells showed significant decrease in proliferation ability. The reduced proliferation after disengagement from ECM suggested that the robust proliferative rate was temporary and there was low risk of tumor formation if using for cartilage repair and regeneration in vivo. Similarly, late-passaged cells in conventional plastic culture system exhibited a decreased proliferative rate, but once contacting with ECM they showed stronger proliferation. The native 3D ECM has the potential to expand aged cells and can be used in some degenerative diseases.

On the other hand, strong chondrogenic differentiation potential is important to MSC-based cartilage repair. In vivo MSCs often stay in a quiescent state but have the potential to self-renew or differentiate rapidly once stimulated by intrinsic or extrinsic factors from the microenvironment. ${ }^{70}$ However, conventional in vitro culture system for MSCs impairs their multipotential. When reaching 70\% - 90\% confluence, MSCs contact with neighbor cells and inhibit their proliferation, so weekly passaging is required in MSCs expansion. ${ }^{18}$ To improve MSC proliferative rate, low seeding density is used to increase the yielded cell number at the cost of long expansion period (usually two to three weeks). ${ }^{16}$ Another method to improve MSC self-renewal is stimulating cells with growth factors such as fibroblast growth factor 2 (FGF-2). However, some studies suggested that the supplement of FGF-2 inhibited MSC chondrogenic potential. ${ }^{71}$ In spite of various means to promote MSC proliferative rate, the intrinsic self-renewal ability of MSCs limits their usage in tissue regeneration. The proliferative rate of MSCs usually significantly decreases after 10 passages and eventually disappears. ${ }^{72}$ Repeated passaging is able to extent the proliferative ability of MSCs for a longer time but seriously impairs the multipotent differentiation. Vacanti et al. compared early-passaged and late-passaged porcine MSCs. The late-passaged MSCs lost their multipotential and only differentiated to adipocytes. Besides, cell aging symptoms appeared in the late-passaged MSCs such as actin accumulation, reduced cell adhesion and high activity of lysosomal acid beta-galactosidase. ${ }^{73}$ The impaired multipotential of MSCs is possibly related to the exposure to enzyme detachment such as trypsin which can degrade cell surface receptors or proteins. $^{73-75}$ 


\subsection{D ECM is a potential positive-isolation model for MSCs}

We chose SDSCs for cartilage regeneration due to their superior chondrogenic differentiation potential to other MSCs such as ADSCs or muscle satellite cells. ${ }^{76}$ To purify SDSCs we used a negative isolation method using Dynabeads conjugated with CD14 antibody to remove type A cells (macrophages); ${ }^{77 ; 78}$ however, this method still failed to separate SDSCs from synovial fibroblasts. Karystinou et al. demonstrated that there were multiple MSC sub-populations in synovium tissue. They obtained 50 colonies from single cell-derived clonal populations and these colonies varied in proliferative doubling time, mean telomere lengths from 5.2 to 10.9 $\mathrm{kb}$ and variable differentiation potency only $30 \%$ of which were adipogenic. ${ }^{79}$ To isolate pure adult stem cells, some new cell surface markers were proposed but they were not popularly accepted such as CD271, CD140b, CD340 and CD349. ${ }^{80}$

In this dissertation, 3D SDSC-deposited ECM is a potential positive-isolation model to isolate or purify MSCs from multiple cell populations. SDSCs or chondrocytes expanded by conventional plastic culture showed diverse expression of cell surface markers, even completely positive but with different fluorescence intensity, suggesting that the conventional expanded cells were a mixed population. But cells expanded on SDSC-deposited ECM exhibited a centralized expression of surface markers and this implied that the cells were composed of one or two major populations (Chapter 2). More importantly, after several passages on ECM this centralization phenomenon appeared more obvious, suggesting that the percentage of the major cell population increased and the yielded cells were likely a unique population instead of a mixture. This phenomenon of centralization expression surface marker in SDSCs was CD90 (Chapter 2) and in articular chondrocyte was CD29 (Chapter 4). However, due to the lack of a unique surface marker for MSCs we can't provide direct evidences for the positive isolation function of ECM.

CD90, originally a marker for thymus-derived lymphocytes, is considered an indicator of chondrogenic potential in SDSCs. Consistent with our study (Chapter 2), Nagase et al. analyzed the ratio of CD90-positive SDSCs and suggested that the ratio decreased with 
passaging accompanied by a reduced chondrogenic potential. ${ }^{81}$ In this dissertation, we found that the ratio of CD90-positive SDSCs increased dramatically when expanding on ECM and it decreased on the conventional plastic culture (Chapter 2). In the expansion of articular chondrocytes (Chapter 4) and nucleus pulpous cells (Chapter 5), the percentage of CD90-positive cells increased and stayed at a high level on ECM, similar to SDSCs, due to the existence of undifferentiated progenitor cells in articular cartilage and nucleus pulpous tissue. However, this tendency did not appear in the expansion of human BMSCs, in which the ratio of CD90-positive cells decreased on ECM (Chapter 6). Overall, CD90 is a potential indicator for chondrogenic differentiation in certain MSCs or progenitor cells and future studies are needed to compare the differences between CD90-positive and CD90-negative cells not only in chondrogenesis but also in multipotent differentiation.

SSEA-4, a stage-specific embryonic antigen, was recently used to identify mesenchymal stem cells and made it as a prospective isolation marker. In human BMSCs, SSEA-4 positive cells had a better colony forming ability and stronger multipotential to chondrogenesis, osteogenesis and adipogenesis. ${ }^{82}$ Interestingly, Lai et al. found human BMSCs after expansion on ECM showed an increased tendency of SSEA-4 positive cells. ${ }^{20}$ We also showed that not only the percentage of SSEA-4 positive cells but also the fluorescence intensity in human BMSCs expanded on ECM was significantly higher than those cultured on conventional plastic flasks (Chapter 6). Overall, SSEA-4 provided a potential way for MSC purification and an indicator for MSC multipotential differentiation ability.

\subsection{MSCs expanded on ECM may have higher potential of integration}

The integration of neo-cartilage tissue into articular cartilage defect sites determined the success of cartilage repair; however, several factors impeded cell-based cartilage regeneration. ${ }^{9 ; 83}$ Physical trauma significantly increased cell death in lesion edges and resulted in a hindering area for neo-cartilage integration. ${ }^{84}$ Due to the absent ability of migration, chondrocytes in neo-cartilage or host cartilage failed to move into lesion edges that hindered the full integration of repaired tissue and defect sites. ${ }^{13 ; 85}$ Archer et al. suggested 
that the intactness of lesion edges was essential to the integration of cartilage defect repair. ${ }^{84}$ The failure of full integration was also related to chondrocyte cell death caused by differentiation-related apoptosis or inflammatory factors. ${ }^{9}$ In vitro chondrogenesis induced by TGF- $\beta 1$ decreased cell viability proven by reduced DNA content. ${ }^{2}$ Using intracellular injection of MSCs or chondrocytes to repair cartilage defect, it required a high cell viability during chondrogenesis. ${ }^{86}$ Conventional expanded cells on plastic culture dramatically lost their cell number but ECM-expanded cells maintained their viability during in vitro chondrogenesis. ${ }^{1 ; 87}$ The high viability of ECM-expanded cells will improve the integration in cartilage repair in vivo and resist the effect of inflammatory factors. In autologous chondrocyte transplantation, dedifferentiated chondrocytes leaded to the failure of cartilage repair because the matrix generated by dedifferentiated chondrocytes was mostly type I collagen that formed fibrous cartilage and degraded eventually. ${ }^{12}$ However, chondrocytes expanded on ECM delayed dedifferentiation and maintained a strong chondrogenic differentiation potential (Chapter 4). The usage of chondrocytes expanded on ECM to repair cartilage defects will be conducted in future. Similarly, nucleus pulpous cells expanded on ECM preserved their original cell phenotype and properties (Chapter 5 ) and can be used in IVD regeneration. ${ }^{88}$

\subsection{D ECM reduces chondrogenic hypertrophic risk in MSCs}

Chondrocyte hypertrophy is an important stage in cartilage and bone development, accompanied by vascularization and calcification. ${ }^{89 ; 90}$ From the initiation of MSC transition toward chondrogenic differentiation, MSCs proceed from type I collagen-positive cells to type II collagen-positive chondrocytes, and changed to type $X$ collagen-positive hypertrophic chondrocytes, terminally became fully differentiated cells expressed MMP13 associated with calcification and ossification. In endochondral bone formation, cartilage was formed first, then chondrocytes underwent a proliferative stage following hypertrophy, change in gene expression and matrix calcification. ${ }^{91}$ In chondrogenic induction in vitro and in vivo, MSCs proceeded hypertrophic transition that changed cell morphology from chondrocyte-like to osteoblasts with the expression of type X collagen, alkaline phosphatase, MMP-13, vascular endothelial growth factor (VEGF) and parathyroid hormone-related protein receptor 
(PTHrPR) ${ }^{92-95}$ The importance of studying chondrocyte hypertrophic mechanism is not only to understand bone development but also to prevent ossification in MSC-based cartilage repair and regeneration.

Pelttari et al. reported that after transplantation of pre-chondrogenic differentiated MSC aggregates into the ectopic sites in SCID mice, they underwent a change to endochondral ossification instead of continuous chondrogenesis, whereas the control group of chondrocyte pellets resisted calcification and vascular invasion. ${ }^{96}$ Moreover, TGF- $\beta^{97}$ and high concentration of dexamethasone ${ }^{98}$ exhibited an inhibitory effect on hypertrophy during MSC chondrogenesis. Meanwhile, during MSC chondrogenic differentiation induced by TGF- $\beta$, the expression of type X collagen and MMP13 increased in the early stage but decreased in the late stage, indicating an intermediate role that was still unclear. ${ }^{94}$ The use of type $\mathrm{X}$ collagen as a marker of chondrocyte hypertrophy may have some limits due to the elevated level in the early chondrogenic stage..$^{93}$

The mechanism of chondrocyte hypertrophy is not fully understood, but the MAPK cascade including ERK1/2 and p38 was suggested to modulate the process of hypertrophy. In the process of BMP-2 induced chondrocyte hypertrophy, characterized by elevated type $\mathrm{X}$ collagen, ERK1/2 might have an inhibitory influence. The inhibition of ERK1/2 induced activation of type $\mathrm{X}$ collagen promotor but had little effect on alkaline phosphatase activity. ${ }^{91}$ Stanton et al. provided evidence that p38 MAP kinase was indispensible in chondrocyte hypertrophic differentiation and the inhibition of p38 resulted in decreased expression of hypertrophic marker genes such as type $\mathrm{X}$ collagen, MMP13 and bone sialoprotein. ${ }^{99}$ However, Prasadam et al. showed the opposite evidence that subchondral bone osteoblasts induced hypertrophic change in articular chondrocytes through ERK1/2 phosphorylation and deactivation of p38 phosphorylation. The inhibition of ERK1/2 significantly suppressed the hypertrophic change. ${ }^{100}$ Different from chondrogenesis, ERK1/2 was continuously activated during osteogenesis. Phosphorylated ERK1/2 activated other transcription factors such as Runx2 and cbfa-1 and induced osteocalcin and mineralization. ${ }^{101-103}$ Considering the elevated level of type X collagen in human BMSCs expanded on ECM (Chapter 6) and the decreased 
activity of ERK1/2, hypertrophic transition was possibly related to the ERK1/2 signaling pathway.

In this dissertation, SDSCs and ADSCs after expansion on ECM showed a suppressed level of hypertrophy-related gene expression and negative immunohistochemistry of type $X$ collagen (Chapter 2 and Chapter 3), suggesting that there was no evidence at protein level to prove the hypertrophic transition of MSCs during in vitro chondrogenesis. With regard to chondrocytes and nucleus pulposus cells expanded on ECM or conventional plastic flasks, there were not positive data for hypertrophic genes or proteins (Chapter 4 and Chapter 5). MSCs from different tissue sources may have distinct potentials of hypertrophy that will be taken into account in future. Furthermore, considering the intrinsic differences between chondrocytes and MSCs in the resistance to hypertrophy, future studies are needed to evaluate MSC chondrogenesis and hypertrophy in native cartilage joints in vivo.

\subsection{ECM Improves chondrogenic potential of MSCs and the underlying mechanism}

The enhanced chondrogenic potential after ECM expansion was proven by different cell types including SDSCs (Chapter 2 and Chapter 7), ADSCs (Chapter 3), BMSCs (Chapter 6), chondrocytes (Chapter 4 and Chapter 7) and nucleus pulposus cells (Chapter 5). The obvious mechanism was the increased expression and responsiveness of TGF- $\beta$ type II receptor (Chapter 6). However, the reason of elevated expression of TGF- $\beta$ type II receptor in human BMSCs after expansion on ECM is quite unclear. The cross-talk between integrin system and TGF- $\beta$ receptors may be involved in this mechanism. Garamszegi et al. recently demonstrated that soluble extracellular matrix proteins can induce Smad signaling that was considered to be activated by TGF- $\beta$ ligand. They showed that soluble collagens and laminins induced Smad activation initially from integrin $\alpha_{2} \beta_{1}$, then recruited TGF- $\beta$ receptors, formed a complex membrane signaling receptor and activated several kinases including FAK and Src. The ECM induced activation of Smads was independent of the activation induced by TGF- $\beta$ ligand. However, they didn't provide the information of the expression or responsiveness of TGF- $\beta$ receptors in the presence of soluble matrix proteins. ${ }^{104}$ 
Decorin is an important component in the cell-deposited ECM, confirmed by Chen et al. using human BMSCs to generate ECM. ${ }^{38}$ Decorin is often found in type I collagen-rich matrices and is composed of a leucine repeated core protein attached with a glycosaminoglycan chain with varied length and composition. Conventionally, decorin was considered an antagonist to TGF- $\beta$ by masking the binding site of TGF- $\beta$ receptors. ${ }^{105 ; 106}$ Before chondrogenic differentiation, the low level of autophosphorylation of TGF- $\beta$ type II receptor in human BMSCs expanded on ECM was contributed by the presence of decorin in ECM (Chapter 6), even though this needs to be confirmed in future. Moreover, decorin can influence integrin-dependent cell adhesion and migration. Fiedler et al. provided evidence that decorin promoted endothelial cell adhesion on fibrillar type I collagen by modulating the activity of integrin $\alpha_{2} \beta_{1}$ instead of $\alpha_{1} \beta_{1}$ and activating insulin-like growth factor-I receptor. ${ }^{107}$ In accordance to our data of an increased expression of integrin $\alpha_{2} \beta_{1}$ after ECM expansion, decorin possibly promoted mesenchymal stem cell adhesion and proliferation through integrin and Rac signaling pathway that is also needed to be explored in future.

In the process of chondrogenic differentiation induced by TGF- $\beta$, non-Smad intracellular signaling pathways shared several kinases of integrin-induced signaling pathways. ERK1/2 were important kinases that could be phosphorylated by integrin activation or TGF- $\beta$ induction. ${ }^{108 ; 109}$ The role of ERK1/2 in chondrogenesis is not completely understood. Watanabe et al. demonstrated that TGF- $\beta$ induced the phosphorylation of ERK1/2 to activate chondrogenic-specific genes expression associated with other transcription factors. ${ }^{110}$ Bobick also provided evidence that the level of phosphorylated ERK1/2 paralleled the progress of human BMSC chondrogenesis; the inhibition of MEK1 or ERK1 significantly attenuated the expression of chondrogenic marker genes. ${ }^{111}$ However, several recent studies suggested that ERK1/2 played an inhibitory role in MSC chondrogenic differentiation. ${ }^{112 ; 113}$ Lee et al. showed the phosphorylated level of ERK1/2 during chondrogenesis in BMSC that reached the peak at 10 hours to 1 day after the induction of TGF- $\beta 3$, but from then until day 14 the activity of ERK1/2 showed a decreased tendency. They suggested that ERK1/2 might play an inhibitory role in MSC chondrogenesis. ${ }^{114}$ Zhao et al. demonstrated that TGF- $\beta$ induced a rapid and 
transient phosphorylation of ERK1/2, whereas activated p38 was in a slow and sustained way. ${ }^{115}$ In this dissertation, we also found a suppressed activity of ERK1/2 in human BMSCs expanded on ECM during chondrogenesis. One possible explanation was that the activation of ERK1/2 initiated the transcription of chondrogenic-specific genes such as aggrecan and type II collagen, but in a long-term induction of chondrogenesis ERK1/2 may give way to other signaling pathways such as PI3K/Akt to generate cartilage-specific matrix.

JNK is another important non-Smad TGF- $\beta$ signaling molecule that was proven to promote cartilage-specific gene expression. ${ }^{108}$ However, Caerteling et al. recently published their work to demonstrate that inhibition of JNK increased chondrogenic gene expression and matrix generation. ${ }^{116}$ The involvement of PI3K-Akt pathway in chondrogenesis was reported. Induced by TGF- $\beta$, Akt was phosphorylated at $\mathrm{T}^{308}$ and $\mathrm{S}^{473}$ by $\mathrm{PI} 3 \mathrm{~K}$ and translocated to the nucleus to mediate the differentiation process. ${ }^{117-120}$ However, the lack of PI3K-Atk analysis is one of the limitations in this dissertation that will be investigated in future. Different from ERK1/2, p38 is considered a positive regulatory protein in chondrogenesis. Oh et al. showed that the activity of p38 increased during chondrogenesis while ERK1/2 decreased. Inhibition of p38 significantly decreased the expression of chondrogenic-specific genes, whereas the blockage of ERK1/2 did not affect. ${ }^{121}$ Meanwhile, Chang et al. provided evidence that PKC promoted MSC chondrogenic differentiation and inhibition of PKC reduced the expression of chondrogenic marker genes. More importantly, the inhibition or depletion of PKC induced sustained activation of ERK1/2 and suppressed chondrogenesis, while inhibition of ERK1/2 enhanced chondrogenesis but it had no effect on PKC. The authors concluded that PKC regulated chondrogenesis through down-regulating the activity of ERK. ${ }^{122}$ However, TGF- $\beta$-induced signaling pathways need to be more investigated in future not only in the in vitro chondrogenesis but also in the microenvironment of in vivo.

Hayashida et al. recently published their work about integrin $\beta_{1}$ and TGF- $\beta$. They showed that in epithelia cells knock-down of integrin $\beta_{1}$ enhanced type I collagen expression via integrin $\alpha_{v} \beta_{3}$ and ERK1/2 signaling pathway stimulated by TGF- $\beta .^{123}$ In this dissertation, MSCs (Chapter 2 and 6) and terminally differentiated cell (Chapter 4 and 5) expanded on ECM 
showed declined expression of integrin $\beta_{1}$ and enhanced chondrogenic potential with high level of type II collagen expression, indicating there possibly was relationship between integrin $\beta_{1}$ and TGF- $\beta$ response. Moreover, Bianchi et al. reported that TGF- $\beta$ upregulated the expression of integrin $\beta_{5}$ in carcinoma cells; the knock-down of integrin $\beta 5$ inhibited epithelial-mesenchymal-transition but it did not affect TGF- $\beta$ target genes. ${ }^{124}$ The interaction of integrin $\beta_{1}$ and TGF- $\beta$ induced chondrogenesis needs to be explored in future work.

In Chapter 4 and 5, ECM showed the effect to increase proliferative rate and delayed dedifferentiation in terminally differentiated cells such as chondrocytes and nucleus pulposus cells. For articular chondrocytes, dedifferentiation underwent during serial monolayer culture with declined expression of type II collagen and aggrecan but increased level of type I collagen. The delayed dedifferentiation process in chondrocytes expanded on ECM was possibly related to PKC and ERK. Yoon et al. showed that the expression of PKCa decreased during chondrocyte dedifferentiation but the overexpression of PKC did not affect the dedifferentiation process. Meanwhile, ERK activity increased during dedifferentiation and inhibition of ERK1/2 successfully retarded chondrocyte dedifferentiation with the elevated expression of type II collagen and proteoglycans. They concluded that PKC and ERK independently regulated chondrocyte dedifferentiation. ${ }^{125}$ Moreover, Barbero et al. demonstrated that dedifferentiated human articular chondrocytes had multipotent differentiation capacity toward chondrogenic, osteogenic and osteogenic lineages; that was believed to be the evidence of progenitor cells or MSCs in articular cartilage. ${ }^{126}$ Future work will be conducted in the interaction between chondrocytes and ECM and mechanism of delaying chondrocyte dedifferentiation.

In this dissertation, we did not observe the improvement in osteogenesis and adipogenesis of SDSCs or ADSCs after ECM expansion. However, Chen et al. demonstrated that human BMSCs expanded on a similar 3D ECM gained stronger osteogenic potential. ${ }^{38}$ The inconsistence of osteogenic potential was possibly because we induced MSCs to osteogenesis or adipogenesis in vitro and they implanted cells subcutaneously into mice after 8-week in vitro osteogenic culture. The in vivo environment could induce cells further 
differentiation with stimulation of certain growth factors and nutrients.

SDSC-deposited ECM not only was able to expand MSCs but also culture differentiated cells including chondrocytes and nucleus pulposus cells. ADSC-deposited ECM showed similar functions to promote MSC self-renewal and chondrogenic potential. Chondrocyte-deposited ECM only improved articular chondrocyte proliferation and redifferentiation. The three types of ECMs had three-dimensional structures but they contained different components. The different compositions in ECM affected cell adhesion, proliferation and differentiation through distinct intracellular signaling pathways. However, SDSC-deposited ECM exhibited a universal compatibility to MSCs and terminally differentiated cells that may have a wide application in tissue regenerative medicine and establish an in vitro model to study cell and matrix interaction.

In conclusion, we demonstrated that 3D cell-deposited ECM provides a proper microenvironment for in vitro expansion of MSCs and terminally differentiated cells, not only promoting self-renewal ability but also improving chondrogenic potential, and the novel 3D expansion system greatly improves cell-based cartilage and intervertebral disc regeneration. 


\section{REFERENCES}

1. Koga H, Engebretsen L, Brinchmann JE et al. Mesenchymal stem cell-based therapy for cartilage repair: a review. Knee.Surg.Sports Traumatol.Arthrosc. 2009;17:1289-97.

2. Pei M, He F, Vunjak-Novakovic G. Synovium-derived stem cell-based chondrogenesis. Differentiation 2008;76:1044-56.

3. Horwitz EM, Prockop DJ, Fitzpatrick LA et al. Transplantability and therapeutic effects of bone marrow-derived mesenchymal cells in children with osteogenesis imperfecta. Nat.Med. 1999;5:309-13.

4. Choi JH, Gimble JM, Lee $\mathrm{K}$ et al. Adipose tissue engineering for soft tissue regeneration. Tissue Eng Part B Rev. 2010;16:413-26.

5. Orlic D, Kajstura J, Chimenti $S$ et al. Bone marrow cells regenerate infarcted myocardium. Nature 2001;410:701-5.

6. Strauer BE, Brehm M, Zeus T et al. Repair of infarcted myocardium by autologous intracoronary mononuclear bone marrow cell transplantation in humans. Circulation 2002;106:1913-8.

7. Ferrari $G$, Cusella-De AG, Coletta $M$ et al. Muscle regeneration by bone marrow-derived myogenic progenitors. Science 1998;279:1528-30.

8. Mezey E, Chandross KJ, Harta G et al. Turning blood into brain: cells bearing neuronal antigens generated in vivo from bone marrow. Science 2000;290:1779-82.

9. Vinatier C, Mrugala D, Jorgensen $C$ et al. Cartilage engineering: a crucial combination of cells, biomaterials and biofactors. Trends Biotechnol. 2009;27:307-14.

10. Lin L, Zhou C, Wei X et al. Articular cartilage repair using dedifferentiated articular chondrocytes and bone morphogenetic protein 4 in a rabbit model of articular cartilage defects. Arthritis Rheum. 2008;58:1067-75.

11. Segers VF, Lee RT. Stem-cell therapy for cardiac disease. Nature 2008;451:937-42.

12. Marlovits $\mathrm{S}$, Zeller $\mathrm{P}$, Singer $\mathrm{P}$ et al. Cartilage repair: generations of autologous chondrocyte transplantation. Eur.J.Radiol. 2006;57:24-31. 
13. Fritz J, Janssen P, Gaissmaier $\mathrm{C}$ et al. Articular cartilage defects in the knee--basics, therapies and results. Injury 2008;39 Suppl 1:S50-S57.

14. Gaissmaier C, Koh JL, Weise K et al. Future perspectives of articular cartilage repair. Injury 2008;39 Suppl 1:S114-S120.

15. Martel-Pelletier J, Boileau C, Pelletier JP et al. Cartilage in normal and osteoarthritis conditions. Best.Pract.Res. Clin.Rheumatol. 2008;22:351-84.

16. Bonab MM, Alimoghaddam $\mathrm{K}$, Talebian $\mathrm{F}$ et al. Aging of mesenchymal stem cell in vitro. BMC. Cell Biol. 2006;7:14.

17. Liu L, DiGirolamo CM, Navarro PA et al. Telomerase deficiency impairs differentiation of mesenchymal stem cells. Exp.Cell Res. 2004;294:1-8.

18. Minguell JJ, Erices A, Conget P. Mesenchymal stem cells. Exp.Biol.Med.(Maywood.) 2001;226:507-20.

19. He F, Chen X, Pei M. Reconstruction of an in vitro tissue-specific microenvironment to rejuvenate synovium-derived stem cells for cartilage tissue engineering. Tissue Eng Part A 2009;15:3809-21.

20. Lai $Y$, Sun $Y$, Skinner CM et al. Reconstitution of Marrow-derived Extracellular Matrix Ex Vivo: a Robust Culture System for Expanding Large-scale Highly Functional Human Mesenchymal Stem Cells. Stem Cells Dev. 2009.

21. Talarmin $\mathrm{H}$, Rescan $\mathrm{C}$, Cariou $\mathrm{S}$ et al. The mitogen-activated protein kinase kinase/extracellular signal-regulated kinase cascade activation is a key signalling pathway involved in the regulation of $\mathrm{G}(1)$ phase progression in proliferating hepatocytes. Mol.Cell Biol. 1999;19:6003-11.

22. Westwick JK, Lambert QT, Clark GJ et al. Rac regulation of transformation, gene expression, and actin organization by multiple, PAK-independent pathways. Mol. Cell Biol. 1997;17:1324-35.

23. Zohn IE, Symons M, Chrzanowska-Wodnicka $M$ et al. Mas oncogene signaling and transformation require the small GTP-binding protein Rac. Mol.Cell Biol. 1998;18:1225-35.

24. Page K, Li J, Hodge JA et al. Characterization of a Rac1 signaling pathway to cyclin D(1) expression in airway smooth muscle cells. J.Biol.Chem. 1999;274:22065-71. 
25. Jirmanova L, Afanassieff M, Gobert-Gosse $S$ et al. Differential contributions of ERK and PI3-kinase to the regulation of cyclin D1 expression and to the control of the G1/S transition in mouse embryonic stem cells. Oncogene 2002;21:5515-28.

26. Rowan S, Fisher DE. Mechanisms of apoptotic cell death. Leukemia 1997;11:457-65.

27. Rollet-Labelle E, Grange MJ, Elbim $\mathrm{C}$ et al. Hydroxyl radical as a potential intracellular mediator of polymorphonuclear neutrophil apoptosis. Free Radic.Biol.Med. 1998;24:563-72.

28. Davis W, Jr., Ronai Z, Tew KD. Cellular thiols and reactive oxygen species in drug-induced apoptosis. J.Pharmacol.Exp.Ther. 2001;296:1-6.

29. Plantin-Carrenard E, Bernard M, Derappe C et al. Differential responses of proliferative and non-proliferative leukemia cells to oxidative stress. Free Radic. Res. 2005;39:1-13.

30. Lamari F, Braut-Boucher F, Pongnimitprasert $\mathrm{N}$ et al. Cell adhesion and integrin expression are modulated by oxidative stress in EA.hy 926 cells. Free Radic.Res. 2007;41:812-22.

31. Barnouin K, Dubuisson ML, Child ES et al. H2O2 induces a transient multi-phase cell cycle arrest in mouse fibroblasts through modulating cyclin D and p21Cip1 expression. J.Biol.Chem. 2002;277:13761-70.

32. Lim JH, Lee YM, Chun YS et al. Reactive oxygen species-mediated cyclin D1 degradation mediates tumor growth retardation in hypoxia, independently of p21cip1 and hypoxia-inducible factor. Cancer Sci. 2008;99:1798-805.

33. Kim TH, Oh S, Kim SS. Recombinant human prothrombin kringle-2 induces bovine capillary endothelial cell cycle arrest at G0-G1 phase through inhibition of cyclin D1/CDK4 complex: modulation of reactive oxygen species generation and up-regulation of cyclin-dependent kinase inhibitors. Angiogenesis. 2005;8:307-14.

34. Philp D, Chen SS, Fitzgerald $W$ et al. Complex extracellular matrices promote tissue-specific stem cell differentiation. Stem Cells 2005;23:288-96.

35. Tsai KS, Kao SY, Wang CY et al. Type I collagen promotes proliferation and osteogenesis of human mesenchymal stem cells via activation of ERK and Akt 
pathways. J.Biomed.Mater.Res.A 2010;94:673-82.

36. Fassett J, Tobolt D, Hansen LK. Type I collagen structure regulates cell morphology and EGF signaling in primary rat hepatocytes through cAMP-dependent protein kinase A. Mol.Biol.Cell 2006;17:345-56.

37. Majd $H$, Wipff PJ, Buscemi L et al. A novel method of dynamic culture surface expansion improves mesenchymal stem cell proliferation and phenotype. Stem Cells 2009;27:200-9.

38. Chen XD, Dusevich $\vee$, Feng JQ et al. Extracellular matrix made by bone marrow cells facilitates expansion of marrow-derived mesenchymal progenitor cells and prevents their differentiation into osteoblasts. J.Bone Miner.Res. 2007;22:1943-56.

39. Cukierman E, Pankov R, Stevens DR et al. Taking cell-matrix adhesions to the third dimension. Science 2001;294:1708-12.

40. Cukierman E, Pankov R, Yamada KM. Cell interactions with three-dimensional matrices. Curr.Opin.Cell Biol. 2002;14:633-9.

41. Even-Ram S, Yamada KM. Cell migration in 3D matrix. Curr.Opin.Cell Biol. 2005;17:524-32.

42. Winer JP, Janmey PA, McCormick ME et al. Bone marrow-derived human mesenchymal stem cells become quiescent on soft substrates but remain responsive to chemical or mechanical stimuli. Tissue Eng Part A 2009;15:147-54.

43. Weber JD, Raben DM, Phillips PJ et al. Sustained activation of extracellular-signal-regulated kinase 1 (ERK1) is required for the continued expression of cyclin D1 in G1 phase. Biochem.J. 1997;326 (Pt 1):61-8.

44. Cary LA, Han DC, Guan JL. Integrin-mediated signal transduction pathways. Histol. Histopathol. 1999;14:1001-9.

45. Zhu X, Assoian RK. Integrin-dependent activation of MAP kinase: a link to shape-dependent cell proliferation. Mol.Biol.Cell 1995;6:273-82.

46. Park JH, Ryu JM, Han HJ. Involvement of caveolin-1 in fibronectin-induced mouse embryonic stem cell proliferation: Role of FAK, RhoA, PI3K/Akt, and ERK 1/2 pathways. J.Cell Physiol 2010.

47. Roovers K, Davey G, Zhu X et al. Alpha5beta1 integrin controls cyclin D1 
expression by sustaining mitogen-activated protein kinase activity in growth factor-treated cells. Mol.Biol.Cell 1999;10:3197-204.

48. Kuwada SK, Li X. Integrin alpha5/beta1 mediates fibronectin-dependent epithelial cell proliferation through epidermal growth factor receptor activation. Mol.Biol.Cell 2000;11:2485-96.

49. Bao W, Stromblad S. Integrin alphav-mediated inactivation of p53 controls a MEK1-dependent melanoma cell survival pathway in three-dimensional collagen. J.Cell Biol. 2004;167:745-56.

50. Sawhney RS, Cookson MM, Omar $Y$ et al. Integrin alpha2-mediated ERK and calpain activation play a critical role in cell adhesion and motility via focal adhesion kinase signaling: identification of a novel signaling pathway. J.Biol.Chem. 2006;281:8497-510.

51. Honore $\mathrm{S}$, Kovacic $\mathrm{H}$, Pichard $\mathrm{V}$ et al. Alpha2beta1-integrin signaling by itself controls G1/S transition in a human adenocarcinoma cell line (Caco-2): implication of NADPH oxidase-dependent production of ROS. Exp.Cell Res. 2003;285:59-71.

52. Inoue O, Suzuki-Inoue K, Dean WL et al. Integrin alpha2beta1 mediates outside-in regulation of platelet spreading on collagen through activation of Src kinases and PLCgamma2. J.Cell Biol. 2003;160:769-80.

53. Priestley GV, Scott LM, Ulyanova T et al. Lack of alpha4 integrin expression in stem cells restricts competitive function and self-renewal activity. Blood 2006;107:2959-67.

54. Zhang $\mathrm{H}$, Li Z, Viklund EK et al. P21-activated kinase 4 interacts with integrin alpha $\mathrm{v}$ beta 5 and regulates alpha $\mathrm{v}$ beta 5 -mediated cell migration. J.Cell Biol. 2002;158:1287-97.

55. Braam SR, Zeinstra L, Litjens $S$ et al. Recombinant vitronectin is a functionally defined substrate that supports human embryonic stem cell self-renewal via alphavbeta5 integrin. Stem Cells 2008;26:2257-65.

56. Castello-Cros R, Khan DR, Simons J et al. Staged stromal extracellular 3D matrices differentially regulate breast cancer cell responses through PI3K and beta1-integrins. BMC.Cancer 2009;9:94. 
57. Song $\mathrm{H}$, Cha $\mathrm{MJ}$, Song BW et al. Reactive oxygen species inhibit adhesion of mesenchymal stem cells implanted into ischemic myocardium via interference of focal adhesion complex. Stem Cells 2010;28:555-63.

58. Yan B, Smith JW. A redox site involved in integrin activation. J.Biol.Chem. 2000;275:39964-72.

59. Chiarugi P, Pani G, Giannoni E et al. Reactive oxygen species as essential mediators of cell adhesion: the oxidative inhibition of a FAK tyrosine phosphatase is required for cell adhesion. J.Cell Biol. 2003;161:933-44.

60. Humphries MJ. Integrin structure. Biochem.Soc.Trans. 2000;28:311-39.

61. Lv X, Su L, Yin D et al. Knockdown of integrin beta4 in primary cultured mouse neurons blocks survival and induces apoptosis by elevating NADPH oxidase activity and reactive oxygen species level. Int.J.Biochem.Cell Biol. 2008;40:689-99.

62. Hayashi $\mathrm{Y}$, Furue MK, Okamoto $\mathrm{T}$ et al. Integrins regulate mouse embryonic stem cell self-renewal. Stem Cells 2007;25:3005-15.

63. Park HB, Golubovskaya V, Xu L et al. Activated Src increases adhesion, survival and alpha2-integrin expression in human breast cancer cells. Biochem.J. 2004;378:559-67.

64. $\mathrm{Xu} \mathrm{J}$, Zutter MM, Santoro SA et al. PDGF induction of alpha 2 integrin gene expression is mediated by protein kinase C-zeta. J.Cell Biol. 1996;134:1301-11.

65. Xu J, Clark RA. A three-dimensional collagen lattice induces protein kinase C-zeta activity: role in alpha2 integrin and collagenase mRNA expression. J.Cell Biol. 1997;136:473-83.

66. Eliceiri BP, Puente XS, Hood JD et al. Src-mediated coupling of focal adhesion kinase to integrin alpha(v)beta5 in vascular endothelial growth factor signaling. J.Cell Biol. 2002;157:149-60.

67. Wang W, Kirsch T. Annexin V/beta5 integrin interactions regulate apoptosis of growth plate chondrocytes. J.Biol.Chem. 2006;281:30848-56.

68. Cardo-Vila M, Arap W, Pasqualini R. Alpha $v$ beta 5 integrin-dependent programmed cell death triggered by a peptide mimic of annexin V. Mol.Cell 2003;11:1151-62. 
69. Schlaepfer DD, Jones J, Haigler HT. Inhibition of protein kinase C by annexin V. Biochemistry 1992;31:1886-91.

70. Kuhn NZ, Tuan RS. Regulation of stemness and stem cell niche of mesenchymal stem cells: implications in tumorigenesis and metastasis. J.Cell Physiol 2010;222:268-77.

71. Hildner F, Peterbauer A, Wolbank S et al. FGF-2 abolishes the chondrogenic effect of combined BMP-6 and TGF-beta in human adipose derived stem cells. J.Biomed.Mater.Res.A 2010;94:978-87.

72. Yoshimura H, Muneta T, Nimura A et al. Comparison of rat mesenchymal stem cells derived from bone marrow, synovium, periosteum, adipose tissue, and muscle. Cell Tissue Res. 2007;327:449-62.

73. Vacanti V, Kong E, Suzuki G et al. Phenotypic changes of adult porcine mesenchymal stem cells induced by prolonged passaging in culture. J.Cell Physiol 2005;205:194-201.

74. Bruder SP, Jaiswal N, Haynesworth SE. Growth kinetics, self-renewal, and the osteogenic potential of purified human mesenchymal stem cells during extensive subcultivation and following cryopreservation. J.Cell Biochem. 1997;64:278-94.

75. Javazon EH, Beggs KJ, Flake AW. Mesenchymal stem cells: paradoxes of passaging. Exp. Hematol. 2004;32:414-25.

76. Sakaguchi $\mathrm{Y}$, Sekiya I, Yagishita $\mathrm{K}$ et al. Comparison of human stem cells derived from various mesenchymal tissues: superiority of synovium as a cell source. Arthritis Rheum. 2005;52:2521-9.

77. Bilgen $\mathrm{B}$, Ren $\mathrm{Y}$, Pei $\mathrm{M}$ et al. CD14-negative isolation enhances chondrogenesis in synovial fibroblasts. Tissue Eng Part A 2009;15:3261-70.

78. Pei M, He F, Kish VL et al. Engineering of functional cartilage tissue using stem cells from synovial lining: a preliminary study. Clin. Orthop.Relat Res. 2008;466:1880-9.

79. Karystinou A, Dell'Accio F, Kurth TB et al. Distinct mesenchymal progenitor cell subsets in the adult human synovium. Rheumatology.(Oxford) 2009;48:1057-64.

80. Buhring $\mathrm{HJ}$, Battula VL, Treml S et al. Novel markers for the prospective isolation of human MSC. Ann.N.Y.Acad.Sci. 2007;1106:262-71. 
81. Nagase T, Muneta T, Ju YJ et al. Analysis of the chondrogenic potential of human synovial stem cells according to harvest site and culture parameters in knees with medial compartment osteoarthritis. Arthritis Rheum. 2008;58:1389-98.

82. Gang EJ, Bosnakovski D, Figueiredo CA et al. SSEA-4 identifies mesenchymal stem cells from bone marrow. Blood 2007;109:1743-51.

83. Khan IM, Gilbert SJ, Singhrao SK et al. Cartilage integration: evaluation of the reasons for failure of integration during cartilage repair. A review. Eur.Cell Mater. 2008;16:26-39.

84. Archer CW, Redman S, Khan I et al. Enhancing tissue integration in cartilage repair procedures. J.Anat. 2006;209:481-93.

85. Hunziker EB. Articular cartilage repair: basic science and clinical progress. A review of the current status and prospects. Osteoarthritis. Cartilage. 2002;10:432-63.

86. Lee $\mathrm{KB}$, Hui $\mathrm{JH}$, Song IC et al. Injectable mesenchymal stem cell therapy for large cartilage defects--a porcine model. Stem Cells 2007;25:2964-71.

87. Heng BC, Cao T, Lee EH. Directing stem cell differentiation into the chondrogenic lineage in vitro. Stem Cells 2004;22:1152-67.

88. Sakai D. Future perspectives of cell-based therapy for intervertebral disc disease. Eur.Spine J. 2008;17 Suppl 4:452-8.

89. Mueller MB, Tuan RS. Functional characterization of hypertrophy in chondrogenesis of human mesenchymal stem cells. Arthritis Rheum. 2008;58:1377-88.

90. Pelttari K, Steck E, Richter W. The use of mesenchymal stem cells for chondrogenesis. Injury 2008;39 Suppl 1:S58-S65.

91. Reilly GC, Golden EB, Grasso-Knight G et al. Differential effects of ERK and p38 signaling in BMP-2 stimulated hypertrophy of cultured chick sternal chondrocytes. Cell Commun.Signal. 2005;3:3.

92. Mwale F, Girard-Lauriault PL, Wang HT et al. Suppression of genes related to hypertrophy and osteogenesis in committed human mesenchymal stem cells cultured on novel nitrogen-rich plasma polymer coatings. Tissue Eng 2006;12:2639-47.

93. Mwale F, Stachura D, Roughley P et al. Limitations of using aggrecan and type X 
collagen as markers of chondrogenesis in mesenchymal stem cell differentiation. J.Orthop.Res. 2006;24:1791-8.

94. Sekiya I, Vuoristo JT, Larson BL et al. In vitro cartilage formation by human adult stem cells from bone marrow stroma defines the sequence of cellular and molecular events during chondrogenesis. Proc.Natl.Acad.Sci.U.S.A 2002;99:4397-402.

95. Yoo JU, Barthel TS, Nishimura $\mathrm{K}$ et al. The chondrogenic potential of human bone-marrow-derived mesenchymal progenitor cells. J.Bone Joint Surg.Am. 1998;80:1745-57.

96. Pelttari $\mathrm{K}$, Winter $\mathrm{A}$, Steck $\mathrm{E}$ et al. Premature induction of hypertrophy during in vitro chondrogenesis of human mesenchymal stem cells correlates with calcification and vascular invasion after ectopic transplantation in SCID mice. Arthritis Rheum. 2006;54:3254-66.

97. Ballock RT, Heydemann A, Wakefield LM et al. TGF-beta 1 prevents hypertrophy of epiphyseal chondrocytes: regulation of gene expression for cartilage matrix proteins and metalloproteases. Dev.Biol. 1993;158:414-29.

98. Leboy PS, Sullivan TA, Nooreyazdan M et al. Rapid chondrocyte maturation by serum-free culture with BMP-2 and ascorbic acid. J.Cell Biochem. 1997;66:394-403.

99. Stanton LA, Sabari S, Sampaio AV et al. p38 MAP kinase signalling is required for hypertrophic chondrocyte differentiation. Biochem.J. 2004;378:53-62.

100. Prasadam I, van GS, Friis T et al. ERK-1/2 and p38 in the regulation of hypertrophic changes of normal articular cartilage chondrocytes induced by osteoarthritic subchondral osteoblasts. Arthritis Rheum. 2010;62:1349-60.

101. Mayer-Wagner S, Schiergens TS, Sievers B et al. Membrane-based cultures generate scaffold-free neocartilage in vitro: influence of growth factors. Tissue Eng Part A 2010;16:513-21.

102. McMahon LA, O'Brien FJ, Prendergast PJ. Biomechanics and mechanobiology in osteochondral tissues. Regen.Med. 2008;3:743-59.

103. Salasznyk RM, Klees RF, Boskey A et al. Activation of FAK is necessary for the osteogenic differentiation of human mesenchymal stem cells on laminin-5. J.Cell Biochem. 2007;100:499-514. 
104. Garamszegi N, Garamszegi SP, Samavarchi-Tehrani P et al. Extracellular matrix-induced transforming growth factor-beta receptor signaling dynamics. Oncogene 2010;29:2368-80.

105. Kinsella MG, Bressler SL, Wight TN. The regulated synthesis of versican, decorin, and biglycan: extracellular matrix proteoglycans that influence cellular phenotype. Crit Rev.Eukaryot.Gene Expr. 2004;14:203-34.

106. Kresse H, Schonherr E. Proteoglycans of the extracellular matrix and growth control. J.Cell Physiol 2001;189:266-74.

107. Fiedler LR, Schonherr E, Waddington $\mathrm{R}$ et al. Decorin regulates endothelial cell motility on collagen I through activation of insulin-like growth factor I receptor and modulation of alpha2beta1 integrin activity. J.Biol.Chem. 2008;283:17406-15.

108. Tuli R, Tuli S, Nandi $S$ et al. Transforming growth factor-beta-mediated chondrogenesis of human mesenchymal progenitor cells involves $\mathrm{N}$-cadherin and mitogen-activated protein kinase and Wnt signaling cross-talk. J.Biol.Chem. 2003;278:41227-36.

109. Murakami S, Kan M, McKeehan WL et al. Up-regulation of the chondrogenic Sox9 gene by fibroblast growth factors is mediated by the mitogen-activated protein kinase pathway. Proc.Natl.Acad.Sci.U.S.A 2000;97:1113-8.

110. Watanabe $\mathrm{H}$, de Caestecker MP, Yamada Y. Transcriptional cross-talk between Smad, ERK1/2, and p38 mitogen-activated protein kinase pathways regulates transforming growth factor-beta-induced aggrecan gene expression in chondrogenic ATDC5 cells. J.Biol.Chem. 2001;276:14466-73.

111. Bobick BE, Matsche Al, Chen FH et al. The ERK5 and ERK1/2 signaling pathways play opposing regulatory roles during chondrogenesis of adult human bone marrow-derived multipotent progenitor cells. J.Cell Physiol 2010;224:178-86.

112. Bobick BE, Kulyk WM. The MEK-ERK signaling pathway is a negative regulator of cartilage-specific gene expression in embryonic limb mesenchyme. J.Biol.Chem. 2004;279:4588-95.

113. Kim HJ, Im GI. The effects of ERK1/2 inhibitor on the chondrogenesis of bone marrow- and adipose tissue-derived multipotent mesenchymal stromal cells. Tissue 
Eng Part A 2010;16:851-60.

114. Lee JW, Kim YH, Kim SH et al. Chondrogenic differentiation of mesenchymal stem cells and its clinical applications. Yonsei Med.J. 2004;45 Suppl:41-7.

115. Li J, Zhao Z, Liu J et al. MEK/ERK and p38 MAPK regulate chondrogenesis of rat bone marrow mesenchymal stem cells through delicate interaction with TGF-beta1/Smads pathway. Cell Prolif. 2010;43:333-43.

116. Caerteling SB, van der Kraan PM, Vitters EL et al. Inhibition of JNK enhances chondrogenesis of mesenchymal progenitor cells by increasing autocrine TGFbeta signaling. Arthritis Rheum. 2010.

117. Oh CD, Chun JS. Signaling mechanisms leading to the regulation of differentiation and apoptosis of articular chondrocytes by insulin-like growth factor-1. J.Biol.Chem. 2003;278:36563-71.

118. Starkman BG, Cravero JD, Delcarlo $M$ et al. IGF-I stimulation of proteoglycan synthesis by chondrocytes requires activation of the PI 3-kinase pathway but not ERK MAPK. Biochem.J. 2005;389:723-9.

119. Ciarmatori S, Kiepe D, Haarmann A et al. Signaling mechanisms leading to regulation of proliferation and differentiation of the mesenchymal chondrogenic cell line RCJ3.1C5.18 in response to IGF-I. J.Mol.Endocrinol. 2007;38:493-508.

120. McMahon LA, Prendergast PJ, Campbell VA. A comparison of the involvement of p38, ERK1/2 and PI3K in growth factor-induced chondrogenic differentiation of mesenchymal stem cells. Biochem.Biophys. Res. Commun. 2008;368:990-5.

121. Oh $\mathrm{CD}$, Chang $\mathrm{SH}$, Yoon $\mathrm{YM}$ et al. Opposing role of mitogen-activated protein kinase subtypes, erk-1/2 and p38, in the regulation of chondrogenesis of mesenchymes. J.Biol.Chem. 2000;275:5613-9.

122. Chang $\mathrm{SH}, \mathrm{Oh} \mathrm{CD}$, Yang $\mathrm{MS}$ et al. Protein kinase $\mathrm{C}$ regulates chondrogenesis of mesenchymes via mitogen-activated protein kinase signaling. J.Biol.Chem. 1998;273:19213-9.

123. Hayashida T, Jones JC, Lee CK et al. Loss of $\{$ beta\}1-integrin enhances TGF-\{beta\}1-induced collagen expression in epithelial cells via increased

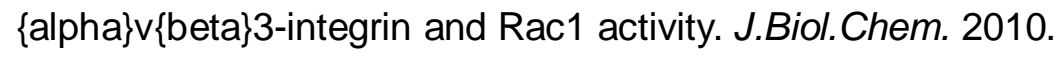


124. Bianchi A, Gervasi ME, Bakin AV. Role of beta5-integrin in epithelial-mesenchymal transition in response to TGFbeta. Cell Cycle 2010;9.

125. Yoon YM, Kim SJ, Oh CD et al. Maintenance of differentiated phenotype of articular chondrocytes by protein kinase $\mathrm{C}$ and extracellular signal-regulated protein kinase. J.Biol.Chem. 2002;277:8412-20.

126. Barbero A, Ploegert S, Heberer $M$ et al. Plasticity of clonal populations of dedifferentiated adult human articular chondrocytes. Arthritis Rheum. 2003;48:1315-25. 